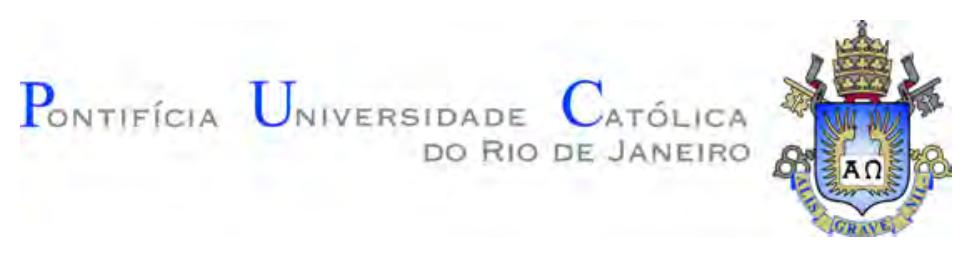

Edson Rodrigo Schlosser

\title{
Análise e Síntese de Redes Refletoras para Aplicações Espaciais
}

\author{
Tese de Doutorado
}

Tese apresentada como requisito parcial para obtenção do grau de Doutor pelo Programa de Pós-Graduação em Engenharia Elétrica do Departamento de Engenharia Elétrica da PUC-Rio.

Orientador : Prof. José Ricardo Bergmann Coorientador: Prof. Marcos Vinício Thomas Heckler 


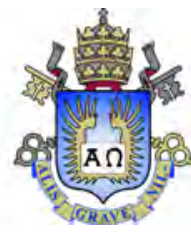

Edson Rodrigo Schlosser

\section{Análise e Síntese de Redes Refletoras para \\ Aplicações Espaciais}

Tese apresentada como requisito parcial para obtenção do grau de Doutor pelo Programa de Pós-Graduação em Engenharia Elétrica da PUC-Rio. Aprovada pela Comissão Examinadora abaixo.

Prof. José Ricardo Bergmann

Orientador

Centro de Estudos em Telecomunicações - PUC-Rio

Prof. Marcos Vinício Thomas Heckler

Coorientador

Universidade Federal do Pampa - UNIPAMPA

Prof. Fernando José da Silva Moreira Universidade Federal de Minas Gerais - UFMG

Maj. Mariana Guimarães Pralon Centro Tecnológico do Exército - CTEx

Prof. Flávio José Vieira Hasselmann Centro de Estudos em Telecomunicações - PUC-Rio

Prof. Guilherme Simon da Rosa

Centro de Estudos em Telecomunicações - PUC-Rio 
Todos os direitos reservados. É proibida a reprodução total ou parcial do trabalho sem autorização da universidade, do autor e do orientador.

\section{Edson Rodrigo Schlosser}

Possui graduação (2007-2011) e mestrado (2011-2014) em Engenharia Elétrica pela Universidade Federal do Pampa (UNIPAMPA). Atualmente, é membro do Laboratório de Eletromagnetismo, Micro-Ondas e Antenas (LEMA). É Professor do Magistério Superior junto ao corpo docente do curso de Engenharia de Telecomunicações na Universidade Federal do Pampa, Campus Alegrete.

Ficha Catalográfica

Schlosser, Edson Rodrigo

Análise e síntese de redes refletoras para aplicações espaciais / Edson Rodrigo Schlosser; orientador: José Ricardo Bergmann; coorientador: Marcos Vinício Thomas Heckler. 2020.

159 f: il. color. ; $30 \mathrm{~cm}$

Tese (doutorado) - Pontifícia Universidade Católica do Rio de Janeiro, Departamento de Engenharia Elétrica, 2020.

Inclui bibliografia

1. Engenharia Elétrica - Teses. 2. Redes refletoras. 3. Método dos momentos. 4. Funções base. 5. Síntese de diagrama de irradiação. I. Bergmann, José Ricardo. II. Heckler, Marcos Vinício Thomas. III. Pontifícia Universidade Católica do Rio de Janeiro. Departamento de Engenharia Elétrica. IV. Título. 


\section{Agradecimentos}

Gostaria de agradecer à Deus pela vida, por minha família e todos os meus amigos;

Aos meus pais Adil e Maria Schlosser, às minhas irmãs Carla e Carmem, e a minha companheira Leilani B. Bruno, pelo incentivo;

Aos meus orientadores Prof. Dr. José R. Bergmann e Prof. Dr. Marcos V. T. Heckler, por toda atenção e suporte científico me concedido no desenvolvimento desta tese;

Ao Programa de Pós-Graduação em Engenharia Elétrica (PPGEE) da Pontifícia Universidade Católica do Rio de Janeiro (PUC-Rio), pelo auxílio na participação em eventos científicos;

Ao Centro de Estudos em Telecomunicações (CETUC) e ao Laboratório de Eletromagnetismo, Micro-ondas e Antenas (LEMA), pela infraestrutura;

À Universidade Federal do Pampa (Unipampa), pelos afastamentos para capacitação profissional;

O presente trabalho foi realizado com apoio da Coordenação de Aperfeiçoamento de Pessoal de Nível Superior - Brasil (CAPES) - Código de Financiamento 001. 


\section{Resumo}

Schlosser, Edson Rodrigo; Bergmann, José Ricardo; Thomas Heckler, Marcos Vinício. Análise e Síntese de Redes Refletoras para Aplicações Espaciais. Rio de Janeiro, 2020. 159p. Tese de Doutorado - Departamento de Engenharia Elétrica, Pontifícia Universidade Católica do Rio de Janeiro.

Neste trabalho é apresentada a análise e síntese de redes refletoras para aplicações espaciais. Redes refletoras são abordadas para expor de forma sucinta suas aplicações, geometrias, métodos de análise e de síntese de diagramas de irradiação. O método do circuito equivalente é utilizado para a formulação da função diádica de Green, possibilitando estabelecer relações entre os campos eletromagnéticos e as densidades superficiais de corrente elétrica em meios estratificados e constituídos por diversas metalizações. Validações numéricas são realizadas através da determinação das constantes de propagação em estruturas slab e guia de onda parcialmente preenchido, formadas por paredes metálicas com condutividade infinita. Análises de redes refletoras com poucos elementos são realizadas empregando-se o método dos momentos para calcular numericamente as densidades superficiais de corrente elétrica que fluem sobre os patches. Adicionalmente, propõe-se a aplicação de uma função base de domínio completo com condição de borda segmentada para modelar o comportamento impulsivo da densidade de corrente nas bordas dos espalhadores. Diferentes curvas de fase são obtidas através da técnica de variação das dimensões físicas dos espalhadores metálicos, considerando o campo elétrico total composto pelos campos espalhado, refletido e difratado, devido às posições espaciais dos patches. Softwares comerciais são utilizados nas verificações dos resultados obtidos. Por fim, o conceito de defasagem progressiva e o método de enxame de partículas foram aplicados para se determinar a fase desejada em cada célula. Assim, as curvas de fase são interpoladas e utilizadas para projetar as dimensões dos elementos impressos e garantir a distribuição de fase calculada sobre a superfície da rede refletora, de forma a reproduzir os diagramas desejados.

\section{Palavras-chave}

Redes refletoras; Método dos momentos; Funções base; Síntese de diagrama de irradiação. 


\section{Abstract}

Schlosser, Edson Rodrigo; Bergmann, José Ricardo (Advisor); Thomas Heckler, Marcos Vinício (Co-Advisor). Analysis and Synthesis of ReflectArrays for Space Applications. Rio de Janeiro, 2020. 159p. Tese de doutorado - Departamento de Engenharia Elétrica, Pontifícia Universidade Católica do Rio de Janeiro.

This thesis presents the analysis and synthesis of reflectarrays for space applications. A review of the state-of-the-art of reflectarrays is presented and the applications, geometries, methods of analysis and methods of synthesis are discussed. Dyadic Green's function is formulated using the equivalent circuit method to describe the relation between electromagnetic fields and electrical current densities in structures composed of stratified media and several metallizations. Slab and partially-filled waveguide formed by metallic walls with infinite conductivity are analyzed to validate the formulation. Analyses of reflectarrays with few elements are performed using the method of moments to numerically calculate the densities of electric current that flow on the patches. Additionally, entire-domain basis function with segmented edge condition to model the impulsive behavior of current density at the edges of the scatterers is proposed. Different phase curves are obtained due to the patches positions in the reflectarray using the variable size technique. Phase curves are calculated considering a total electric field composed of scattered, reflected and diffracted fields. Commercial software is used to verify the obtained results. Finally, the desired phase in each cell is determined using the progressive phase concept and the particle swarm optimisation method. The phase curves are used to design the dimensions of the printed elements, and, thus, ensure the desired phase distribution on the surface of the reflectarrays, and, consequently, the specified radiation pattern.

\section{Keywords}

Reflectarrays; Method of moments; Basis function; Synthesis of the radiation patterns. 


\section{Sumário}

1 Introdução $\quad 22$

2 Contextualização do estado-da-arte para redes refletoras $\quad 27$

2.1 Geometria de espalhadores 28

$\begin{array}{ll}2.2 \text { Variações estruturais } & 29\end{array}$

2.3 Modelamento da fonte 30

2.4 Métodos de análise de redes refletoras 30

2.5 Síntese de redes refletoras 32

2.6 Erros na modelagem de RA 33

2.7 Contribuições deste trabalho 34

3 Formulação da função diádica de Green para estruturas planares $\quad 37$

3.1 Solução da equação de onda 37

3.2 Campos eletromagnéticos 39

3.2.1 Relações dos campos eletromagnéticos Ey em função de Ex no domínio espacial 39

3.2.2 Relações dos campos eletromagnéticos Ez em função de Ex no domínio espacial 40

3.2.3 Relações dos campos eletromagnéticos Ex em função de Ey no domínio espacial $\quad 41$

3.2.4 Relações dos campos eletromagnéticos no domínio espectral 42

3.2.5 Campos eletromagnéticos nas interfaces de uma camada dielétrica 43

3.2.6 Dedução da função de Green para estrutura com múltiplas camadas e metalização $\quad 45$

3.3 Validação numérica 53

4 Análise de estruturas planares a partir do método dos momentos - MoM 57

4.1 Campo elétrico espalhado $\quad 57$

4.1.1 Mapeamento e integração no plano complexo 61

4.1.2 Rede refletora 66

4.1.3 Funções base 67

4.2 Campos elétricos incidente e refletido 73

$\begin{array}{ll}\text { 4.3 Campo elétrico difratado } & 79\end{array}$

4.4 Campo elétrico irradiado $\quad 82$

4.5 Validações numéricas $\quad 84$

4.5.1 Campo elétrico espalhado por um elemento simples 85

4.5.2 Campo elétrico espalhado por redes refletoras 90

4.5.3 Campo elétrico espalhado e refletido por redes refletoras 98

4.5.4 Campo elétrico espalhado, refletido e difratado por redes refletoras 111

$5 \quad$ Síntese de redes refletoras $\quad 123$

5.1 Síntese de rede refletora com apontamento de feixe $\quad 125$

5.2 Síntese de rede refletora com feixe conformado 131 
6 Conclusão e trabalhos futuros

140

Referências bibliográficas

142

A Formulação da função diádica de Green desnormalizada

154

B Estudo da segmentação para a função base com condição de borda

155 


\section{Lista de figuras}

Figura 1.1 Sistema Brasileiro de Coleta de Dados Ambientais (SBCDA).

Figura 1.2 Sistema de comunicação. 24

Figura 1.3 Antena refletora clássica. 24

$\begin{array}{lll}\text { Figura } 1.4 & \text { Rede refletora. } & 25\end{array}$

Figura 2.1 Fluxograma dos tipos de ajustabilidade da fase em RAs. 28

Figura 3.1 Estratificação de uma estrutura plana. 44

Figura 3.2 Campos eletromagnéticos tangenciais e modelo circuital. 48

Figura 3.3 Meios estratificados separados por uma metalização e modelo simplificado.

Figura 3.4 Estrutura de uma rede refletora multicamada e modelo simplicado.

Figura 3.5 Modelos circuitais de estrutura formada por camada(s) dielétrica(s), metalização e diferentes terminações (aberto ou com plano de terra). a) Stripline; b) Plano de terra na interface inferior e região superior aberta; c) Plano de terra na interface superior e região inferior aberta; d) Ambas as interfaces abertas;

e) Admitâncias equivalentes vistas pela fonte.

Figura 3.6 Geometria formada por plano de terra, substrato e ar. $\quad 54$

Figura 3.7 Modelo circuital para a estrutura da Figura 3.6. 54

Figura 3.8 Constantes de propagação de ondas de superfície $\left(\varepsilon_{r}=\right.$ 2, 55). Comparação com os resultados extraídos de [81].

Figura 3.9 Guia retangular parcialmente preenchido.

Figura 3.10 Modelo circuital para a estrutura da Figura 3.9.

Figura 3.11 Constantes de propagação em guia retangular particialmente preenchido. Comparação com os resultados extraídos de $[82]$.

Figura 4.1 Campo elétrico tangencial total sobre a superfície metálica impressa.

Figura 4.2 Modelo circuital para a estrutura da Figura 4.1. $\quad 58$

Figura 4.3 Exemplo de plano complexo para estrutura planar com três modos de propagação. Adaptado de [83].

Figura 4.4 Quatro primeiros modos de expansão em $x$ no domínio completo para um espalhador centralizado com dimensão normalizada.

Figura 4.5 Condição de borda sem segmentação para uma distribuição de corrente no sentido de $x$.

Figura 4.6 Condição de borda com segmentação para uma distribuição de corrente no sentido de $x$ aplicando diferentes valores de $\kappa$.

Figura 4.7 Reflexão em uma interface que separa dois meios distintos. 73

$\begin{array}{lll}\text { Figura 4.8 Reflexão no substrato e plano de terra. } & 76\end{array}$ 
Figura 4.9 Incidência sobre as metalizações. b) Normal; b) Oblíqua. 78

Figura 4.10 Difração na borda do plano de terra. $\quad 80$

Figura 4.11 Elemento simples. $\quad 85$

Figura 4.12 Densidade de corrente superficial para funções base senoidal. a) $J_{x}(x, y)$; b) $J_{y}(x, y)$.

Figura 4.13 Campo calculado e simulado para funções base senoidal.

a) Plano E: $E_{\theta}\left(\theta, \phi=0^{\circ}\right)$; b) Plano $\mathrm{H}: E_{\phi}\left(\theta, \phi=90^{\circ}\right)$.

Figura 4.14 Densidade de corrente superficial para funções base com condição de borda sem segmentação. a) $J_{x}(x, y)$; b) $J_{y}(x, y)$.

Figura 4.15 Campo calculado e simulado para funções base com condição de borda sem segmentação. a) Plano E: $E_{\theta}\left(\theta, \phi=0^{\circ}\right)$; b) Plano H: $E_{\phi}\left(\theta, \phi=90^{\circ}\right)$.

Figura 4.16 Densidade de corrente superficial para funções base com condição de borda segmentada, $\kappa=0$, 4. a) $J_{x}(x, y)$; b) $J_{y}(x, y)$.

Figura 4.17 Campo calculado e simulado para funções base com condição de borda segmentada, $\kappa=0,4$. a) Plano E: $E_{\theta}(\theta, \phi=$ $\left.0^{\circ}\right)$; b) Plano $\mathrm{H}: E_{\phi}\left(\theta, \phi=90^{\circ}\right)$.

Figura 4.18 Curvas de fase para as diferentes funções base com $W=L .89$

Figura 4.19 Curvas de amplitude para as diferentes funções base com $W=L$.

Figura 4.20 Rede $3 \times 3$ com elementos de mesma dimensão física.

Figura 4.21 Rede $5 \times 5$ com elementos de mesma dimensão física.

Figura 4.22 Rede $7 \times 7$ com elementos de mesma dimensão física.

91

91

Figura 4.23 Campo calculado e simulado para uma rede $3 \times 3$ - onda plana com incidência normal. a) Plano $\mathrm{E}: E_{\theta}\left(\theta, \phi=0^{\circ}\right)$; b) Plano H: $E_{\phi}\left(\theta, \phi=90^{\circ}\right)$.

Figura 4.24 Campo calculado e simulado para uma rede $3 \times 3$ - onda plana com incidência oblíqua $\left(\theta_{i}=10^{\circ}\right)$. Plano E: $E_{\theta}\left(\theta, \phi=0^{\circ}\right)$.

Figura 4.25 Campo calculado e simulado para uma rede $3 \times 3$ - onda plana com incidência oblíqua $\left(\theta_{i}=20^{\circ}\right)$. Plano E: $E_{\theta}\left(\theta, \phi=0^{\circ}\right)$.

Figura 4.26 Campo calculado e simulado para uma rede $5 \times 5$ - onda plana com incidência normal. a) Plano $\mathrm{E}$ : $E_{\theta}\left(\theta, \phi=0^{\circ}\right)$; b) Plano H: $E_{\phi}\left(\theta, \phi=90^{\circ}\right)$.

Figura 4.27 Campo calculado e simulado para uma rede $5 \times 5$ - onda plana com incidência oblíqua $\left(\theta_{i}=10^{\circ}\right)$. Plano E: $E_{\theta}\left(\theta, \phi=0^{\circ}\right)$.

Figura 4.28 Campo calculado e simulado para uma rede $5 \times 5$ - onda plana com incidência oblíqua $\left(\theta_{i}=20^{\circ}\right)$. Plano E: $E_{\theta}\left(\theta, \phi=0^{\circ}\right)$.

Figura 4.29 Campo calculado e simulado para uma rede $7 \times 7$ - onda plana com incidência normal $\left(\theta_{i}=0^{\circ}\right)$. a) Plano E: $E_{\theta}(\theta, \phi=$ $\left.0^{\circ}\right)$; b) Plano $\mathrm{H}: E_{\phi}\left(\theta, \phi=90^{\circ}\right)$.

Figura 4.30 Campo calculado e simulado para uma rede $7 \times 7$ - onda plana com incidência oblíqua $\left(\theta_{i}=10^{\circ}\right)$. Plano E: $E_{\theta}\left(\theta, \phi=0^{\circ}\right)$.

Figura 4.31 Campo calculado e simulado para uma rede $7 \times 7$ - onda plana com incidência oblíqua $\left(\theta_{i}=20^{\circ}\right)$. Plano E: $E_{\theta}\left(\theta, \phi=0^{\circ}\right)$.

Figura 4.32 Definição de células. a) Célula isolada; b) Célula central e demais células.

Figura 4.33 Escalonamento para correção de desvio em frequência. 
Figura 4.34 Comportamento da fase das células de uma RA $3 \times 3$ para incidência normal e observação broadside.

Figura 4.35 Comportamento da fase das células de uma RA $5 \times 5$ para incidência normal e observação broadside.

Figura 4.36 Comportamento da fase das células de uma RA $7 \times 7$ para incidência normal e observação broadside.

Figura 4.37 Diferença de fase das células de uma RA $3 \times 3$ em relação a célula central.

Figura 4.38 Diferença de fase das células de uma RA $5 \times 5$ em relação a célula central.

Figura 4.39 Diferença de fase das células de uma RA $7 \times 7$ em relação a célula central.

Figura 4.40 Comparativo das curvas de fase de células centrais de redes $3 \times 3,5 \times 5$ e $7 \times 7$.

Figura 4.41 Diferença de fase da célula isolada e central de redes $3 \times 3$ e $5 \times 5$ para a célula central de uma RA $7 \times 7$.

Figura 4.42 Campo calculado e simulado para um elemento simples - onda plana com incidência normal. a) Plano E: $E_{\theta}\left(\theta, \phi=0^{\circ}\right)$; b) Plano H: $E_{\phi}\left(\theta, \phi=90^{\circ}\right)$.

Figura 4.43 Campo calculado e simulado para uma rede $3 \times 3$ - onda plana com incidência normal. a) Plano E: $E_{\theta}\left(\theta, \phi=0^{\circ}\right)$; b) Plano H: $E_{\phi}\left(\theta, \phi=90^{\circ}\right)$.

Figura 4.44 Campo calculado e simulado para uma rede $3 \times 3$ - onda plana com incidência oblíqua $\left(\theta_{i}=10^{\circ}\right)$. Plano E: $E_{\theta}\left(\theta, \phi=0^{\circ}\right)$.

Figura 4.45 Campo calculado e simulado para uma rede $3 \times 3$ - onda plana com incidência oblíqua $\left(\theta_{i}=20^{\circ}\right)$. Plano E: $E_{\theta}\left(\theta, \phi=0^{\circ}\right)$.

Figura 4.46 Campo calculado e simulado para uma rede $5 \times 5$ - onda plana com incidência normal. a) Plano E: $E_{\theta}\left(\theta, \phi=0^{\circ}\right)$; b) Plano H: $E_{\phi}\left(\theta, \phi=90^{\circ}\right)$.

Figura 4.47 Campo calculado e simulado para uma rede $5 \times 5$ - onda plana com incidência oblíqua $\left(\theta_{i}=10^{\circ}\right)$. Plano $\mathrm{E}$ : $E_{\theta}\left(\theta, \phi=0^{\circ}\right)$.

Figura 4.48 Campo calculado e simulado para uma rede $5 \times 5$ - onda plana com incidência oblíqua $\left(\theta_{i}=20^{\circ}\right)$. Plano E: $E_{\theta}\left(\theta, \phi=0^{\circ}\right)$.

Figura 4.49 Campo calculado e simulado para uma rede $7 \times 7$ - onda plana com incidência normal. a) Plano $\mathrm{E}$ : $E_{\theta}\left(\theta, \phi=0^{\circ}\right)$; b) Plano $\mathrm{H}: E_{\phi}\left(\theta, \phi=90^{\circ}\right)$.

Figura 4.50 Campo calculado e simulado para uma rede $7 \times 7$ - onda plana com incidência oblíqua $\left(\theta_{i}=10^{\circ}\right)$. Plano E: $E_{\theta}\left(\theta, \phi=0^{\circ}\right)$.

Figura 4.51 Campo calculado e simulado para uma rede $7 \times 7$ - onda plana com incidência oblíqua $\left(\theta_{i}=20^{\circ}\right)$. Plano E: $E_{\theta}\left(\theta, \phi=0^{\circ}\right)$.

Figura 4.52 Definição das bordas de células pertencentes a periferia de redes refletoras.

Figura 4.53 Comportamento da fase das células de uma RA $3 \times 3$ com a inclusão de efeitos difrativos.

Figura 4.54 Comportamento da fase das células de uma RA $5 \times 5$ com a inclusão de efeitos difrativos.

Figura 4.55 Comportamento da fase das células de uma RA $7 \times 7$ com a inclusão de efeitos difrativos. 
Figura 4.56 Diferença de fase das células de uma RA $3 \times 3$ para a célula central.

Figura 4.57 Diferença de fase das células de uma RA $5 \times 5$ para a célula central.

Figura 4.58 Diferença de fase das células de uma RA $7 \times 7$ para a célula central.

Figura 4.59 Campo calculado e simulado para uma rede $3 \times 3$ - onda plana com incidência normal. a) Plano E: $E_{\theta}\left(\theta, \phi=0^{\circ}\right)$; b) Plano H: $E_{\phi}\left(\theta, \phi=90^{\circ}\right)$.

Figura 4.60 Campo calculado e simulado para uma rede $3 \times 3$ - onda plana com incidência oblíqua $\left(\theta_{i}=10^{\circ}\right)$. Plano E: $E_{\theta}\left(\theta, \phi=0^{\circ}\right)$.

Figura 4.61 Campo calculado e simulado para uma rede $3 \times 3$ - onda plana com incidência oblíqua $\left(\theta_{i}=20^{\circ}\right)$. Plano E: $E_{\theta}\left(\theta, \phi=0^{\circ}\right)$.

Figura 4.62 Campo calculado e simulado para uma rede $5 \times 5$ - onda plana com incidência normal. a) Plano E: $E_{\theta}\left(\theta, \phi=0^{\circ}\right)$; b) Plano H: $E_{\phi}\left(\theta, \phi=90^{\circ}\right)$.

Figura 4.63 Campo calculado e simulado para uma rede $5 \times 5$ - onda plana com incidência oblíqua $\left(\theta_{i}=10^{\circ}\right)$. Plano E: $E_{\theta}\left(\theta, \phi=0^{\circ}\right)$.

Figura 4.64 Campo calculado e simulado para uma rede $5 \times 5$ - onda plana com incidência oblíqua $\left(\theta_{i}=20^{\circ}\right)$. Plano $\mathrm{E}: E_{\theta}\left(\theta, \phi=0^{\circ}\right)$.

Figura 4.65 Campo calculado e simulado para uma rede $7 \times 7$ - onda plana com incidência normal. a) Plano $\mathrm{E}$ : $E_{\theta}\left(\theta, \phi=0^{\circ}\right)$; b) Plano $\mathrm{H}: E_{\phi}\left(\theta, \phi=90^{\circ}\right)$.

Figura 4.66 Campo calculado e simulado para uma rede $7 \times 7$ - onda plana com incidência oblíqua $\left(\theta_{i}=10^{\circ}\right)$. Plano E: $E_{\theta}\left(\theta, \phi=0^{\circ}\right)$.

Figura 4.67 Campo calculado e simulado para uma rede $7 \times 7$ - onda plana com incidência oblíqua $\left(\theta_{i}=20^{\circ}\right)$. Plano $\mathrm{E}: E_{\theta}\left(\theta, \phi=0^{\circ}\right)$.

Figura 5.1 Fluxograma da síntese de diagramas de re-irradiação e validação.

Figura 5.2 Rede $5 \times 5$ com elementos de dimensões físicas apresentadas na Tabela 5.3.

Figura 5.3 Rede $7 \times 7$ com elementos de dimensões físicas apresentadas na Tabela 5.6.

Figura 5.4 Campo calculado e simulado para uma rede $5 \times 5$ - onda plana com incidência normal. Plano E: $E_{\theta}\left(\theta, \phi=0^{\circ}\right)$.

Figura 5.5 Campo calculado e simulado para uma rede $7 \times 7$ - onda plana com incidência normal. Plano E: $E_{\theta}\left(\theta, \phi=0^{\circ}\right)$.

Figura 5.6 Campo calculado e simulado para uma rede $5 \times 5$ após correção pontual nas amplitudes dos campos elétricos dos patches - onda plana com incidência normal. Plano E: $E_{\theta}(\theta, \phi=$ $\left.0^{\circ}\right)$.

Figura 5.7 Campo calculado e simulado para uma rede $7 \times 7$ após correção pontual nas amplitudes dos campos elétricos dos patches - onda plana com incidência normal. Plano E: $E_{\theta}(\theta, \phi=$ $\left.0^{\circ}\right)$.

Figura 5.8 Diagramas de re-irradiação com contorno. a) RA 5×5; b) RA $7 \times 7$. 
Figura 5.9 Diagramas de re-irradiação 3D com apontamento para $\theta_{b}=20^{\circ}$ e $\phi_{b}=0^{\circ}$. a) RA $5 \times 5$; b) RA $7 \times 7$.

Figura 5.10 Campo calculado e simulado para uma rede $5 \times 5$ - onda plana com incidência normal. a) Plano $\mathrm{E}$ : $E_{\theta}\left(\theta, \phi=0^{\circ}\right)$; b) Plano H: $E_{\phi}\left(\theta, \phi=90^{\circ}\right)$.

Figura 5.11 Campo calculado e simulado para uma rede $7 \times 7$ - onda plana com incidência normal. a) Plano E: $E_{\theta}\left(\theta, \phi=0^{\circ}\right)$; b) Plano H: $E_{\phi}\left(\theta, \phi=90^{\circ}\right)$.

Figura 5.12 Campo calculado e simulado para uma rede $5 \times 5$ após correções pontuais - onda plana com incidência normal. a) Plano E: $E_{\theta}\left(\theta, \phi=0^{\circ}\right)$; b) Plano $\mathrm{H}: E_{\phi}\left(\theta, \phi=90^{\circ}\right)$.

Figura 5.13 Campo calculado e simulado para uma rede $7 \times 7$ após correções pontuais - onda plana com incidência normal. a) Plano E: $E_{\theta}\left(\theta, \phi=0^{\circ}\right)$; b) Plano H: $E_{\phi}\left(\theta, \phi=90^{\circ}\right)$.

Figura 5.14 Diagramas de re-irradiação com contorno. a) RA $5 \times 5$; b) RA $7 \times 7$.

Figura 5.15 Diagramas de re-irradiação 3D conformado. a) RA 5×5; b) RA $7 \times 7$.

Figura B.1 Densidade de corrente superficial para funções base com condição de borda segmentada, $\kappa=0,8$. a) $J_{x}(x, y)$; b) $J_{y}(x, y) .155$

Figura B.2 Campo calculado e simulado para funções base com condição de borda segmentada, $\kappa=0,8$. a) Plano E: $E_{\theta}(\theta, \phi=$ $\left.0^{\circ}\right)$; b) Plano $\mathrm{H}: E_{\phi}\left(\theta, \phi=90^{\circ}\right)$.

Figura B.3 Densidade de corrente superficial para funções base com condição de borda segmentada, $\kappa=0,6$. a) $J_{x}(x, y)$; b) $J_{y}(x, y) .157$

Figura B.4 Campo calculado e simulado para funções base com condição de borda segmentada, $\kappa=0,6$. a) Plano E: $E_{\theta}(\theta, \phi=$ $\left.0^{\circ}\right)$; b) Plano $\mathrm{H}: E_{\phi}\left(\theta, \phi=90^{\circ}\right)$.

Figura B.5 Densidade de corrente superficial para funções base com condição de borda segmentada, $\kappa=0,2$. a) $J_{x}(x, y)$; b) $J_{y}(x, y) .158$

Figura B.6 Campo calculado e simulado para funções base com condição de borda segmentada, $\kappa=0,2$. a) Plano E: $E_{\theta}(\theta, \phi=$ $\left.0^{\circ}\right)$; b) Plano $\mathrm{H}: E_{\phi}\left(\theta, \phi=90^{\circ}\right)$. 


\section{Lista de tabelas}

Tabela 2.1 Número de elementos $\times$ espaçamento. 34

Tabela 4.1 Número de variáveis desconhecidas a serem determinadas a partir do MoM. 98

Tabela 5.1 Numerações das células de uma rede refletora $5 \times 5$. 125

Tabela 5.2 Fases que devem ser reproduzidas pelas células em graus

Tabela 5.3 Tamanhos dos patches quadrados em milímetros.

126

Tabela 5.4 Numerações das células de uma rede refletora $7 \times 7$. 126

Tabela 5.5 Fases que devem ser reproduzidas pelas células em graus $\left({ }^{\circ}\right)$.

Tabela 5.6 Tamanhos dos patches quadrados em milímetros.

Tabela 5.7 Variáveis que relacionam o algoritmo de otimização com a natureza.

Tabela 5.8 Fases que devem ser reproduzidas pelas células em graus $\left({ }^{\circ}\right)$.

Tabela 5.9 Tamanhos dos patches quadrados em milímetros.

Tabela 5.10 Fases que devem ser reproduzidas pelas células em graus $\left({ }^{\circ}\right)$.

Tabela 5.11 Tamanhos dos patches quadrados em milímetros. 


\section{Lista de Abreviaturas}

CMCD - Centro de armazenamento e processamento

ECM - Modelo circuital equivalente

FDTD - Diferenças finitas no domínio do tempo

FEM - Método dos elementos finitos

FFT - Transformada rápida de Fourier

FRA - Rede refletora envolvida, do inglês Folded ReflectArray

GA - Algoritmo genético

GO - Óptica geométrica

GTD - Teoria geométrica da difração

GTOD - Técnica de otimização direta generalizada

IA - Abordagem de interseção

INPE - Instituto Nacional de Pesquisas Espaciais

LP - Periodicidade local

LT - Linha de transmissão

MEC - Método das correntes equivalentes de borda

MEMS - Sistemas microeletromecânicos

MoM - Método dos momentos

PCD - Plataforma de coleta de dados ambientais

PEC - Condutor elétrico perfeito

PO - Óptica física

PSO - Otimização por enxame de partículas

PTD - Teoria física da difração

RA - Rede Refletora, do inglês ReflectArray

RNA - Redes neurais artificiais

RWG - Rao-Wilton-Glisson

SBCDA - Sistema brasileiro de coleta de dados ambientais

SD - Domínio espectral

SDMoM - Método dos momentos no domínio espectral

SEA - Abordagem de elemento cercado

TE - Transversal elétrico

TEM - Transversal eletromagnético

TM - Transversal magnético

UHF - Frequência ultra-alta

UTD - Teoria uniforme da difração 
$A$ - Dimensão do plano de terra e substrato na direção $x$

$B$ - Dimensão do plano de terra e substrato na direção $y$

$\overline{\tilde{B}}_{b}$ - Submatriz $2 \times 2$ que relaciona os campos magnéticos no domínio espectral

$C$ - Borda do plano de terra

$C_{x}$ - Dimensão da célula em $x$

$C_{y}$ - Dimensão da célula em $y$

$d B$ - Decibel

$d_{b}$ - Espessura física do substrato

$\bar{d}_{b}$ - Espessura física normalizada do substrato

$d_{i}$ - Espessura física da $i$-ésima estratificação

$\bar{d}_{i}$ - Espessura física normalizada da $i$-ésima estratificação

$D_{e}^{I, f}$ - Coeficiente de difração, campo elétrico, corrente elétrica (franja)

$D_{h}^{I, f}$ - Coeficiente de difração, campo magnético, corrente elétrica (franja)

$D_{h}^{M, f}$ - Coeficiente de difração, campo magnético, corrente magnética (franja)

$D_{e}^{I, P O}$ - Coeficiente de difração, campo elétrico, corrente elétrica (PO)

$D_{h}^{I, P O}$ - Coeficiente de difração, campo magnético, corrente elétrica (PO)

$D_{h}^{M, P O}$ - Coeficiente de difração, campo magnético, corrente magnética (PO)

$E_{x}$ - Campo elétrico na direção $x$ no domínio espacial

$E_{y}$ - Campo elétrico na direção $y$ no domínio espacial

$E_{z}$ - Campo elétrico na direção $z$ no domínio espacial

E - Vetor campo elétrico no domínio espacial

$\mathbf{E}^{(e s p)}$ - Vetor campo elétrico espalhado por uma metalização

$\mathbf{E}^{(i n c)}$ - Vetor campo elétrico incidente em uma metalização

$\mathbf{E}^{(d)}$ - Vetor campo elétrico difratado na borda

$\mathbf{E}^{(f)}$ - Vetor campo elétrico da fonte

$\mathbf{E}^{(F W C)}$ - Vetor campo elétrico difratado na borda devido a corrente de

franja

$\mathbf{E}^{(P O C)}$ - Vetor campo elétrico difratado devido as correntes da PO na borda

do plano de terra

$\mathbf{E}^{(r)}$ - Vetor campo elétrico refletido

$\mathbf{E}^{(t)}$ - Vetor campo elétrico transmitido

$\mathbf{E}^{(T)}$ - Vetor campo elétrico total sobre uma metalização

$\tilde{E}_{x}$ - Campo elétrico na direção $x$ no domínio espectral

$\tilde{E}_{y}$ - Campo elétrico na direção $y$ no domínio espectral

$\tilde{E}_{z}$ - Campo elétrico na direção $z$ no domínio espectral

$\tilde{\mathbf{E}}$ - Vetor campo elétrico no domínio espectral

erfc - Função erro complementar

$\tilde{\mathbf{F}}_{i}$ - Vetor contendo os campos eletromagnéticos tangenciais na $i$-ésima inter-

face no domínio espectral 
$F_{e c}-$ Função transição

$f_{r}$ - frequência de ressonância

$\tilde{\mathbf{F}}_{i-1}$ - Vetor contendo os campos eletromagnético tangenciais na $i$-ésima -1

interface no domínio espectral

$\mathscr{F}($.$) - Transformada de Fourier da função (.)$

$\overline{\tilde{G}}$ - Função diádica de Green

$\mathscr{H}_{0}$ - Função Struve de ordem zero

$H_{x}$ - Campo magnético na direção $x$ no domínio espacial

$H_{y}$ - Campo magnético na direção y no domínio espacial

$H_{z}$ - Campo magnético na direção $z$ no domínio espacial

H - Vetor campo magnético no domínio espacial

$\mathbf{H}^{(f)}$ - Vetor campo magnético da fonte

$\mathbf{H}^{(r)}$ - Vetor campo magnético refletido

$\mathbf{H}^{(t)}$ - Vetor campo magnético transmitido

$\tilde{H}_{x}$ - Campo magnético na direção $x$ no domínio espectral

$\tilde{H}_{y}$ - Campo magnético na direção $x$ no domínio espectral

$\tilde{H}_{z}$ - Campo magnético na direção $x$ no domínio espectral

$\tilde{\mathbf{H}}$ - Vetor campo magnético no domínio espectral

$I_{n}$ - Coeficiente da função base

$I_{n_{x}}$ - Coeficiente da função base na direção $x$

$I_{n_{y}}$ - Coeficiente da função base na direção $y$

I - Vetor corrente (coeficientes) do método dos momentos

$j$ - Raiz quadrada de -1

$J_{x}$ - Densidade de corrente elétrica superficial na coordenada $x$

$J_{y}$ - Densidade de corrente elétrica superficial na coordenada $y$

$\mathbf{J}^{(f)}$ - Vetor densidade de corrente elétrica superficial na fonte

$\mathbf{J}^{(s)}$ - Vetor densidade de corrente elétrica superficial na metalização

$\mathbf{J}_{m}$ - Função teste vetorial para a densidade de corrente superficial

$\mathbf{J}_{n}$ - Função base vetorial para a densidade de corrente superficial

$\tilde{J}_{x}$ - Densidade de corrente elétrica superficial na coordenada $x$ no domínio espectral

$\tilde{J}_{y}$ - Densidade de corrente elétrica superficial na coordenada y no domínio espectral

$\tilde{\mathbf{J}}^{(s)}$ - Densidade de corrente superficial sobre o patch no domínio espectral

$J_{0}$ - Função de Bessel de primeira espécie e ordem zero

$k_{0}$ - Constante de propagação no espaço livre

$k_{x}$ - Constante de propagação na direção $x$

$k_{y}$ - Constante de propagação na direção $y$

$k_{z}$ - Constante de propagação na direção $z$ 
$\overline{\tilde{K}}_{i}$ - Matriz híbrida de um $i$-ésimo meio estratificado

$\overline{\tilde{K}}_{a}$ - Matriz equivalente acima da metalização

$\overline{\tilde{K}}_{b}$ - Matriz equivalente abaixo da metalização

$\overline{\tilde{K}}_{e q}$ - Matriz equivalente para um meio estratificado

$k_{\bar{x}}$ - Constante de propagação normalizada na direção $x$

$k_{\bar{y}}$ - Constante de propagação normalizada na direção $y$

$k_{\bar{z}}$ - Constante de propagação normalizada na direção $z$

$L$ - Largura da metalização na direção $x$

$\mathscr{L}_{0}$ - Função Struve modificada de ordem zero

$N_{x}$ - Número de modos na coordenada $x$

$N_{y}$ - Número de modos na coordenada $y$

$R$ - Coeficiente de reflexão generalizado

$R_{c b}$ - Raio de curvatura da borda

r - Vetor posição

$S$ - Superfície do patch

$s_{E}$ - Distância do ponto de difração para o ponto de observação

s - Vetor distância entre fonte e ponto de observação

s' - Vetor distância entre fonte e ponto de difração

$t$ - Tempo

$T$ - Coeficiente de transmissão

V - Vetor tensão (excitação) do método dos momentos

$\overline{\tilde{V}}_{b}$ - Submatriz $2 \times 2$ que relaciona os campos elétricos no domínio espectral

$w$ - Frequência angular

$W$ - Largura da metalização na direção y

$x$ - Coordenada do sistema de coordenadas cartesiano

$x^{\prime}$ - Posição da fonte na coordenada $x$

$x^{(o)}$ - Posição central do patch na coordenada $x$

$\bar{x}$ - Coordenada normalizada do sistema de coordenadas cartesiano

$y$ - Coordenada do sistema de coordenadas cartesiano

$y^{\prime}$ - Posição da fonte na coordenada $y$

$y^{(o)}$ - Posição central do patch na coordenada $y$

$\bar{y}$ - Coordenada normalizada do sistema de coordenadas cartesiano

$\overline{\tilde{y}}$ - Submatriz $2 \times 2$ que relaciona a derivada do vetor campo magnético

com o campo elétrico (no domínio espectral)

$\overline{\tilde{Y}}^{(a)}$ - Admitância total acima de uma metalização vista a partir dos terminais da fonte

$\overline{\tilde{Y}}^{(b)}$ - Admitância total abaixo de uma metalização vista a partir dos termi-

nais da fonte

$\overline{\tilde{Y}}^{(t)}$ - Admitância total vista a partir dos terminais da fonte 
$\overline{\tilde{Y}}_{0}$ - Admitância de uma camada inferior aberta

$\overline{\tilde{Y}}_{n+1}$ - Admitância de uma camada superior aberta

$\overline{\tilde{Y}}_{b}$ - Submatriz $2 \times 2$ que relaciona o campo elétrico com o campo magnético

no domínio espectral

$z$ - Coordenada do sistema de coordenadas cartesiano

$z^{\prime}$ - Posição da fonte na coordenada $z$

$\bar{z}$ - Coordenada normalizada do sistema de coordenadas cartesiano

$\bar{Z}$ - Matriz impedância do método dos momentos

$\overline{\tilde{Z}}_{b}$ - Submatriz $2 \times 2$ que relaciona o campo magnético com o campo

elétrico no domínio espectral

$\theta$ - Coordenada do sistema de coordenadas esférico

$\theta^{\prime}$ - Coordenada da fonte no sistema de coordenadas esférico

$\theta_{b}$ - Ângulo do feixe principal na coordenada $\theta$

$\theta_{i}$ - Ângulo de incidência dos campos eletromagnéticos na coordenada $\theta$

$\theta_{r}$ - Ângulo de reflexão dos campos eletromagnéticos incidentes na coordenada $\theta$

$\theta_{t}$ - Ângulo de transmissão dos campos eletromagnéticos na coordenada $\theta$

$\phi$ - Coordenada do sistema de coordenadas esférico

$\phi^{\prime}$ - Coordenada da fonte no sistema de coordenadas esférico

$\phi_{b}$ - Direção do feixe principal na coordenada $\phi$

$\phi_{i}$ - Ângulo de incidência dos campos eletromagnéticos na coordenada $\phi$

$\phi_{r}$ - Ângulo de reflexão dos campos eletromagnéticos incidentes na coordenada $\phi$

$\phi_{t}$ - Ângulo de transmissão dos campos eletromagnéticos na coordenada $\phi$

$\phi_{0}^{\prime}$ - Ângulo entre vetor tangente ao plano de terra e vetor incidência

$\phi_{0}$ - Ângulo entre vetor tangente ao plano de terra e vetor observação

$r$ - Coordenada do sistema de coordenadas esférico

$\eta_{0}$ - Impedância intrínseca do espaço livre

$\kappa$ - Fator de segmentação da função base com borda segmentada

$\varepsilon$ - Permissividade elétrica

$\varepsilon_{0}$ - Permissividade elétrica do espaço livre

$\varepsilon_{r}$ - Permissividade relativa do material

$\lambda$ - Comprimento de onda

$\lambda_{0}$ - Comprimento de onda no espaço livre

$\mu$ - Permeabilidade magnética

$\mu_{0}$ - Permeabilidade magnética do espaço livre

$\beta_{0}^{\prime}$ - Ângulo entre vetor tangente a borda e vetor incidência

$\beta_{0}$ - Ângulo entre vetor tangente a borda e vetor observação

$\otimes$ - Produto matricial de Kronecker 
|| - Componente paralela

$\perp$ - Componente perpendicular

$\Gamma$ - Coeficiente de reflexão

$\delta($.$) - Função delta de Dirac$

res[.] - Resíduo da função [.]

$\operatorname{sgn}($.$) - Função sinal (.)$

$\langle$.$\rangle - Produto interno$

- - Graus 
Ouve o canto gauchesco e brasileiro Desta terra que eu amei desde guri Flor de tuna, camocim de mel campeiro Pedra moura das quebradas do Inhanduy

Antonio Augusto Fagundes, Canto Alegretense. 


\section{Introdução}

As áreas de tecnologia espacial e de comunicação são consideradas relevantes pelos países desenvolvidos, em que grandes investimentos em pesquisas são disponibilizados constantemente para o desenvolvimento e o aprimoramento destes sistemas. O Brasil tem investido recursos para aperfeiçoar sua capacidade no setor de produção de satélites, visando aplicações em defesa, comunicações e monitoramento ambiental. Atualmente, o Brasil conta com uma rede de plataformas de coleta de dados ambientais (PCDs), distribuídas em todo o território nacional, que são monitoradas pelo Instituto Nacional de Pesquisas Espaciais (INPE). Algumas destas estruturas são aplicadas em ambientes remotos e de difícil acesso, podendo-se citar as estações responsáveis pela cobertura da Floresta Amazônica. As plataformas instaladas possuem sensores capazes de medir grandezas físicas do local de interesse, tais como temperatura, umidade, velocidade dos ventos, etc. Estas estruturas possibilitam monitorar as mudanças climáticas que estes ambientes vêm sofrendo cotidianamente. A coleta destes dados é realizada através de uma constelação de nanossatélites, dedicados a receber os sinais transmitidos pelos sensores alocados nestes ambientes e a retransmiti-los para os centros de armazenamento e processamento (CMCD). Esse conjunto de PCDs, juntamente com o segmento espacial, é conhecido como Sistema Brasileiro de Coleta de Dados Ambientais (SBCDA) e pode ser visualizado na Figura 1.1.

O sistema embarcado nos nanossatélites contém o bloco de recepção (uplink do transponder), responsável pelo enlace de subida de dados e que opera em UHF, e de retransmissão (downlink do transponder), que opera em Banda S e estabelece o enlace de descida entre o satélite e estações terrenas de armazenamento e processamento dos dados coletados, conforme ilustrado na Figura 1.2. O sistema de radiofrequência é composto por antenas e circuitos de recepção e transmissão, constituídos por amplificadores de baixo ruído, filtros, amplificadores de potência, misturadores e outros componentes eletrônicos que operam na faixa de micro-ondas.

Neste trabalho, tem-se apenas o interesse na modelagem eletromagnética de uma antena a ser utilizada no link de descida, que pode operar com determinada faixa na banda $\mathrm{S}(2 \mathrm{GHz}$ a $4 \mathrm{GHz})$. Diversos tipos de antenas são 
apresentados na literatura, tais como monopolos, dipolos, cornetas, microfita, parabólicas e outras derivações. Entretanto, deve-se dedicar atenção especial na escolha, pois estas devem ser integradas ao corpo do nanossatélite no momento do lançamento (cubo com arestas de $20 \mathrm{~cm}$ ). Em [1] são discutidas as vantagens e desvantagens em relação à máxima abertura, ganho, apontamento, largura de banda, nível dos lóbulos secundários, acondicionamento, complexidade, confiabilidade, massa e custos para três tipos de antenas que podem ser aplicadas em nanossatélites, que são as antenas refletoras, as redes de microfita e as redes refletoras.

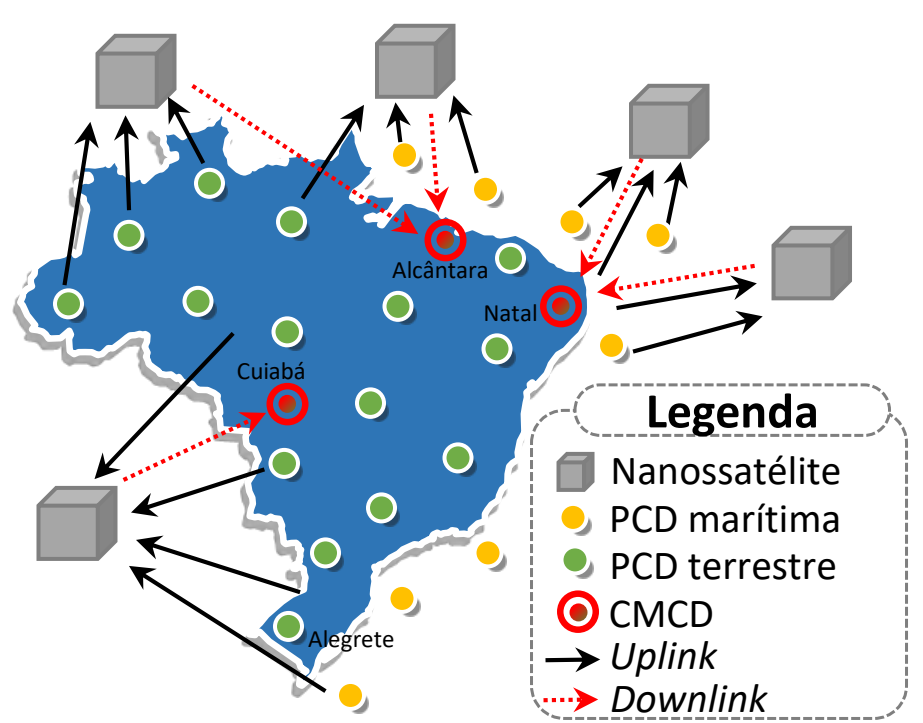

Figura 1.1: Sistema Brasileiro de Coleta de Dados Ambientais (SBCDA).

Antenas refletoras clássicas são compostas de um alimentador, geralmente utilizam uma antena corneta, colocado no foco ou com offset do parabolóide e responsável por iluminar a superfície metálica com uma onda plana [2], conforme ilustrado na Figura 1.3. A partir disso, o campo elétrico é refletido pelo refletor, cujo formato é descrito a partir de um diagrama de irradiação. Para determinadas aplicações, principalmente no que se refere à cobertura espacial ou de telefonia celular, um diagrama de irradiação conformado pode ser desejado [3,4], em que a superfície do refletor deve ser modelada para se obter tal característica. Entretanto, a construção desta superfície é complexa e exige aproximações, o que pode causar imprecisão nos resultados obtidos para a antena projetada. Estruturas de grande porte apresentam dificuldades de manufaturamento e transporte devido ao tamanho físico e a elevada massa, sendo, em muitos casos, necessária a confecção em partes para posteriormente serem montadas. Adicionalmente, no que se refere à cobertura espacial, a integração destas antenas em satélites de pequeno porte (nanossatélites) é inviável no momento do lançamento devido às limitações do modelo físico. Para con- 


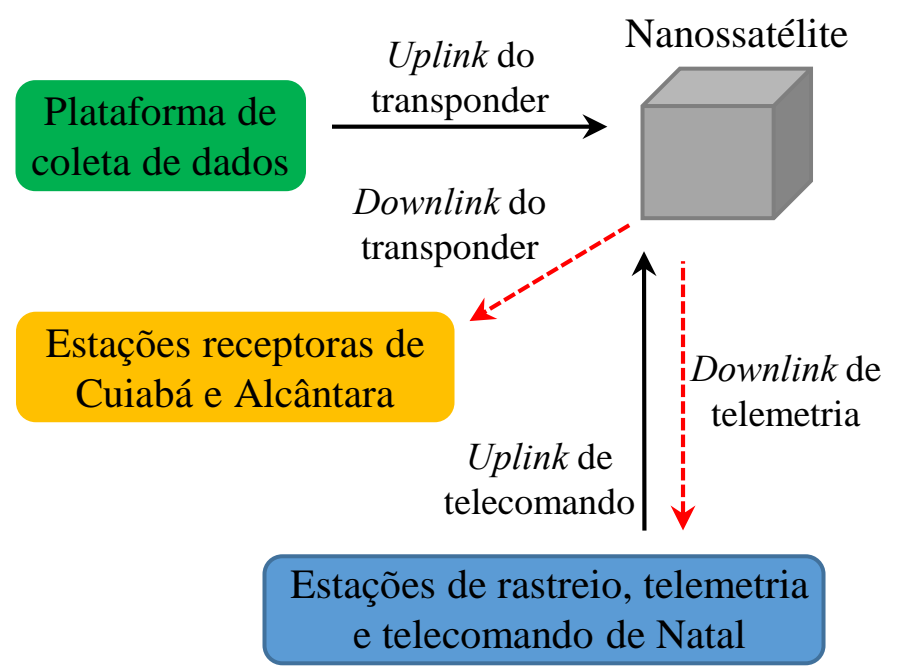

Figura 1.2: Sistema de comunicação.

tornar este problema, antenas refletoras em malha podem ser aplicadas, pois permitem o fechamento do parabolóide no momento do lançamento, porém a possibilidade de conformação de feixe é perdida. Além disso, a aplicação deste tipo de antena provocaria o sombreamento das demais antenas responsáveis pelo uplink do transponder e uplink/downlink da telemetria e telecomando.
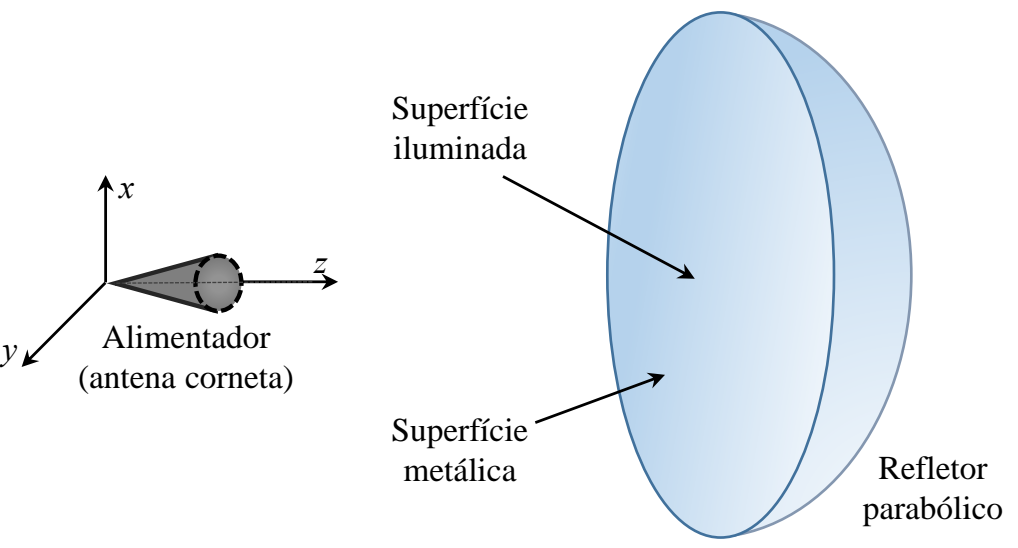

Figura 1.3: Antena refletora clássica.

Nos últimos anos, através da evolução da engenharia de materiais, o desenvolvimento de compostos com baixas perdas na faixa de micro-ondas, além de alta resistência e estabilidade mecânica, têm permitido o desenvolvimento de substratos que possibilitam o projeto de antenas impressas com alta eficiência. Fazendo uso deste tipo de tecnologia, uma alternativa às antenas refletoras tradicionais consiste no emprego de uma rede de antenas em microfita. Entretanto, a abertura da antena é limitada às dimensões físicas do nanossatélite e 
o sistema alimentador apresenta grande complexidade de projeto para elevado número de patches.

Outra opção é a substituição da superfície metálica do refletor convencional por uma rede de antenas em tecnologia de microfita, denominada rede refletora (do inglês, ReflectArray - RA), como pode ser visualizada na Figura 1.4. Este tipo de estrutura é formada por espalhadores metálicos, impressos sobre dielétrico de baixa perda e alta rigidez mecânica, pode ser multicamada [5], e possui um plano de terra. As RAs apresentam facilidade de construção através de uma prototipadora de precisão, baixo peso e custo de fabricação, facilidade de integração com a estrutura do nanossatélite [6], além de possuir ganho e eficiência de irradiação adequados quando substratos com baixas perdas na faixa de micro-ondas são aplicados. A grande vantagem em relação aos refletores tradicionais (clássicos e malháveis) é a possibilidade da conformação sobre as estruturas no momento do lançamento.
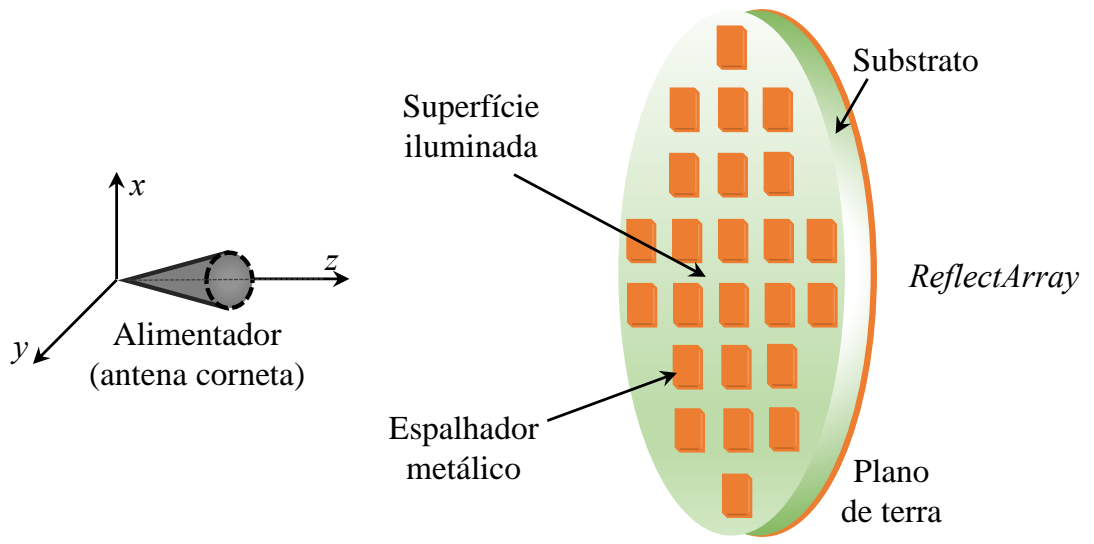

Figura 1.4: Rede refletora.

Neste contexto, este trabalho busca apresentar uma abordagem para a modelagem eletromagnética de redes refletoras.

Este trabalho está organizado em capítulos da seguinte forma,

Capítulo 2: Contextualização teórica sobre os tipos de RAs, as geometrias de espalhadores, o modelamento da fonte, os métodos de análise e de síntese de redes refletoras, erros na modelagem e a proposta desta tese;

Capítulo 3: Formulação da função diádica de Green para estruturas planares a partir do método do circuito equivalente. As estratificações são representadas por matrizes híbridas e a estrutura física é modelada através de um circuito equivalente, obtendo-se assim uma relação entre o campo elétrico e a densidade de corrente superficial, ambas no domínio espectral; 
Capítulo 4: Aplicação das condições de contorno sobre a metalização e a formulação do método dos momentos - MoM para se determinar a densidade de corrente superficial e o campo elétrico espalhado por elementos simples e redes refletoras. A análise de estruturas planares é realizada fazendo-se uso do método dos momentos e da função diádica de Green determinada no Capítulo 3. A aplicação de funções base com condição de borda segmentada é proposto para reduzir o número de modos para representar o comportamento impulsivo da densidade de corrente superficial nas bordas dos patches. Adicionalmente, os campos elétricos incidente, refletido e difratado são abordados e inclusos no cálculo do campo elétrico total re-irradiado;

Capítulo 5: Sintetização de diagramas de irradiação através da defasagem progressiva e de método heurístico. A partir da determinação da fase, projetamse os tamanhos dos elementos impressos necessários para reproduzirem os diagramas de irradiação desejados com base em diferentes curvas de fase. $\mathrm{O}$ conceito de defasagem progressiva entre as células da rede é aplicado para determinar analiticamente a fase desejada para obter-se um apontamento de feixe em determinada direção. Também, o método de enxame de partículas é utilizado para determinar numericamente as fases que reproduzam um diagrama com feixe retangular nos planos $\mathrm{E}$ e $\mathrm{H}$;

Capítulo 6: Discussões sobre a metodologia de análise e síntese de redes refletoras com base na modelagem eletromagnética desenvolvida na tese, resultados apresentados e trabalhos futuros na temática de redes refletoras. 


\section{2}

\section{Contextualização do estado-da-arte para redes refletoras}

A grande maioria dos projetos propostos pela literatura apresenta uma rede refletora plana, com geometria circular, quadrada, retangular ou hexagonal, mas também pode ser curva ${ }^{1}$. Os elementos impressos sobre o dielétrico podem ser dipolos lineares, dipolos cruzados e patches com diferentes formatos (retangular, circular, etc.) que podem possuir fendas.

O diagrama de irradiação de redes refletoras é obtido a partir da soma vetorial do campo espalhado por cada elemento da rede, adicionado da contribuição da reflexão ocorrida no plano de terra/substrato e da difração de borda. Este campo elétrico total, avaliado em campo distante, pode apresentar determinado formato. Para isto, a amplitude e a fase do campo produzido por cada elemento devem ser definidas. Em [7] é apresentada a possibilidade do controle da amplitude a partir do uso de resistores, embora essa prática não seja comum em virtude de introduzir perdas e, consequentemente, reduzir o ganho da rede. Os trabalhos apresentados na literatura concentram-se apenas na otimização da fase [6].

A grande desvantagem deste tipo de antena é a banda estreita, limitada a partir de dois parâmetros: i) largura de operação do elemento impresso; ii) diferença de fase entre os diversos caminhos da fonte aos espalhadores metálicos. O item i) é o fator principal para a limitação de banda de RAs pequenas, enquanto que ii) prevalece para RAs com dimensões maiores que $50 \lambda_{0}$ e pequena razão entre a distância focal e o tamanho do refletor [6].

Técnicas para alargamento da banda de operação foram propostas ao longo dos anos para amenizar o problema da faixa estreita em antenas impressas em tecnologia de microfita. Algumas destas consistem na proposta de diferentes topologias, em que fendas podem ser inseridas no patch para gerar mais de uma frequência de ressonância. Com este mesmo intuito, outra técnica consiste em empilhar patches a partir de estrutura multicamada [6].

Os caminhos entre a fonte e as superfícies metálicas impressas apresentam diferentes comprimentos elétricos (relacionada às distâncias físicas), assim sofrem variações de fase em função da frequência, fazendo com que o diagrama

\footnotetext{
${ }^{1}$ Substrato flexível conformado sobre uma superfície. Exemplo: cilíndrica.
} 
de irradiação da antena modifique. Para resolver este problema, em [8] é proposta uma RA curva.

Diversos parâmetros podem ser explorados em projetos de redes refletoras, tais como tamanho, formato, orientação e posição dos elementos na rede. Adicionalmente, o número de camadas da estrutura, o(s) tipo(s) de substrato(s) e o formato da superfície do plano de terra podem ser investigados.

\section{1}

\section{Geometria de espalhadores}

Os elementos impressos sobre o dielétrico podem apresentar diversos formatos geométricos, os quais se diferenciam em termos de variação de fase, eficiência, banda e frequência de operação, polarização e reconfigurabilidade.

A fase desejada em cada elemento de RAs pode ser alcançada a partir das seguintes características:

i) Elemento com tamanho fixo: variada através de inserção de linhas de transmissão conectadas nos elementos de tamanho fixo, ou através de modificações estruturais, compensando, assim, a diferença de fase entre a fonte e os patches com diferentes posições espaciais, e atribuindo a distribuição de fase desejada ao longo da rede refletora;

ii) Elemento com tamanho variável: modificando a dimensão física de cada irradiador, uma pequena defasagem é introduzida na frequência de ressonância, o que modifica a fase do campo espalhado por cada elemento;

iii) Rotação dos elementos: modificada a partir da disposição com rotação angular dos elementos na rede;

iv) Elemento com reconfigurabilidade: ajustada em tempo real a partir de artifícios eletrônicos ou microeletromecânicos.

As possibilidades descritas podem ser observadas na Figura 2.1.

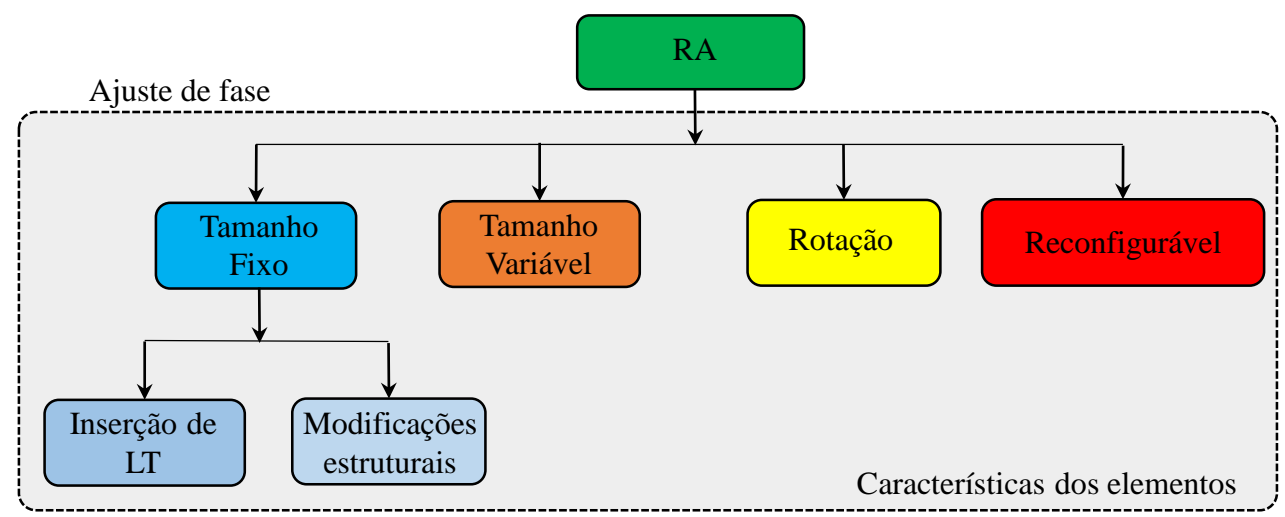

Figura 2.1: Fluxograma dos tipos de ajustabilidade da fase em RAs. 
A inserção de linhas de transmissão possibilita modificar a fase do campo irradiado por elementos de tamanho fixo, necessitando apenas determinar o comprimento correspondente a fase desejada, conforme abordado em [9-13]. Outra possibilidade para elementos de dimensão fixa é a aplicação de fenda/recortes na metalização impressa, podendo-se citar os formatos fragmentados propostos em [14,15], estruturas fractais em [16-18] Phoenix em [19-21], loop em [22-24], Archimedes em [25] e slot em [26-28].

Elementos com formato quadrado, elíptico e dipolos impressos também são vastamente aplicados em projeto de redes refletoras com ajuste de fase através da variação do tamanho da metalização, conforme apresentado em [29-32].

A técnica de elementos rotacionados foi aplicada nos projetos de [33-38], e a combinação desta técnica com a de elementos de tamanho variado em [39].

Em [40] é apresentado o projeto de um sistema reconfigurável eletromecânico que consiste na rotação de elementos a partir de micromotores acoplados aos espalhadores e que são controlados por um dispositivo lógico. Outro caminho para a reconfigurabilidade de RA é através da utilização de componentes eletrônicos, tais como diodos PIN ou varactor [41-47] e transistores [48]. Conforme mencionado em [49-52], também pode ser alcançada através de sistemas microeletromecânicos (MEMS) e a partir do uso de materiais funcionais (cristal líquido ou filmes finos).

\section{2}

\section{Variações estruturais}

$\mathrm{Na}$ literatura, existem alguns projetos que destoam em relação às topologias clássicas de redes refletoras. Em [53-55] a estrutura é composta de um alimentador posicionado a certa distância de uma RA, que é formada apenas pelo plano de terra e um substrato com furos cilíndricos (não existem patches).

Em [56] é apresentada uma RA para aplicações em THz, em que a estrutura é formada por um plano de terra e dielétrico com diversos níveis de altura.

Já em [57-61] é apresentada uma RA envolvida, do inglês Folded ReflectArray - FRA, em que a antena corneta é colocada com a abertura no centro da RA, sendo os elementos iluminados pelo campo refletido por uma superfície inserida acima do alimentador. 


\section{3 \\ Modelamento da fonte}

No projeto de RAs pode-se assumir a iluminação dos diversos patches por uma onda plana. Outra possibilidade é a representação do campo elétrico da fonte através de uma função $\cos ^{q}$ ou feixe Gaussiano, conforme apresentado em [62], em que boa concordância com as medidas em campo distante pode ser observada. Um alimentador que possui determinada banda de operação, diagrama de irradiação, ganho e polarização também pode ser utilizado para esta finalidade, podendo-se citar as antenas cornetas, conforme apresentado em [31], ou de microfita, aplicada em um projeto para nanossatélite em [1].

Em [63] a síntese e projeto de um alimentador é apresentado para maximizar a eficiência da abertura, melhorando a polarização cruzada, o decaimento e o spillover ${ }^{2}$. Em [64] a medida do campo próximo de uma antena corneta foi utilizada para aumentar a confiabilidade da análise. O impacto do erro de fase na região dos lóbulos laterais do diagrama de irradiação de uma RA foi apresentado em [65].

Alguns estudos abordam a posição espacial do alimentador com relação à superfície espalhadora de RAs. Em [66] são mostrados os ganhos na faixa de operação de uma rede com banda larga em função do deslocamento da posição do alimentador sobre o eixo focal. Em [67] é apresentado o efeito do deslocamento de feixe em função da variação da frequência (do inglês, beam squint) para uma dada posição com offset. Para corrigir o desvio de feixe, em [68] é proposta a utilização de duas fontes com offset, alinhadas e em lados opostos da RA. Entretanto, o uso de múltiplas fontes pode provocar o surgimento de grating lobes em diagramas com feixe único, sendo em [69] apresentada uma solução para este fenômeno. Já em [70] o projeto de uma estrutura para operar em dupla faixa (banda Ku e Ka) e dupla polarização é apresentado, sendo, neste caso, utilizados dois alimentadores independentes.

\section{4}

\section{Métodos de análise de redes refletoras}

Em [71] são apresentados dois métodos utilizados inicialmente para análise de redes refletoras planas. A primeira consiste na teoria clássica de redes de antenas para determinar o campo elétrico irradiado. No segundo, o método do campo na abertura é discutido, em que os campos espalhados são determinados a partir do princípio de equivalência. Nenhuma destas abordagens considera o efeito do acoplamento mútuo entre os elementos da rede, o que causa imprecisão nos resultados obtidos.

${ }^{2}$ campo irradiado para fora da superfície da RA. 
Outra possibilidade é o emprego de técnicas numéricas de onda completa, tais como o método das diferenças finitas no domínio do tempo (do inglês, finite-difference time-domain - FDTD), método dos momentos (do inglês, method of moments - MoM) e método dos elementos finitos (do inglês, finite element method - FEM) [6].

O MoM tem sido bastante aplicado na análise de RAs para se calcular de forma eficiente as densidades de correntes elétricas superficiais ao longo dos elementos metálicos, incluindo os efeitos de acoplamento mútuo e as ondas de superfície. A técnica citada pode apresentar considerável esforço computacional no preenchimento das matrizes envolvidas para redes com elevado números de elementos e de modos.

Para reduzir o esforço computacional, a abordagem da periodicidade local (do inglês, local periodicity - LP) foi proposta em [72]. Neste método cada patch impresso da rede é analisado assumindo-se que o elemento individual faça parte de uma rede infinita de elementos idênticos. Adicionalmente, a técnica da LP considera um plano de terra infinito. Apesar das observações apresentadas, o método fornece resultado eficiente e tem sido amplamente utilizado no projeto de redes impressas de grande porte (dimensões superiores a $15 \times 15$ espalhadores). Vale destacar que RAs reais possuem plano de terra truncado e não são periódicas. Desta forma, a consideração da periodicidade local pode causar erros, principalmente em projetos contendo pequeno número de elementos.

O MoM no domínio espectral faz uso da função diádica de Green, sendo em [73] apresentada a formulação para estruturas com múltiplas camadas e metalização em uma interface arbitrária.

Equações básicas de projeto para sub-redes refletoras simétricas são derivadas e validadas em [74]. Em [75] redes neurais artificiais (RNA) são utilizadas para caracterizar a resposta eletromagnética dos elementos de uma RA formada por multicamadas. O treinamento da RNA foi realizado aplicando o algoritmo back propagation, tendo como entrada os seguintes parâmetros: ângulo de incidência, frequência e seis variáveis geométricas livres. Os resultados obtidos apresentam boa concordância em relação àqueles calculados numericamente com MoM, exigindo tempo computacional inferior.

Uma técnica de otimização direta generalizada (GTOD), baseada no método dos momentos no domínio espectral, com periodicidade local e algoritmo de otimização minimax, foi proposta em [76] para a análise de redes com elementos irregularmente espaçados e orientados.

Em [77] uma técnica denominada de abordagem de elemento cercado (do inglês, surrounded element approach - SEA) foi apresentada. Nesta, o elemento 
é analisado na presença de elementos adjacentes não idênticos, possibilitando a inclusão aproximada do acoplamento mútuo. Como resultado, a fase de reflexão modificada devido a não periodicidade pode ser contabilizada.

Ferramentas comerciais têm sido amplamente utilizadas na análise de redes refletoras, tais como Designer, HFSS, CST e FEKO. Em [78], o HFSS foi aplicado no diagnóstico do aparecimento de lóbulo intenso no campo espalhado por uma RA composta de elementos de tamanho variado e espaçados de $0,5 \lambda_{0}$. Como solução, redes com elementos mais próximos (aproximadamente $0,35 \lambda_{0}$ ) foram sugeridas.

O CST foi utilizado em [79] para validar o projeto de uma RA com espaçamento não uniforme, modelada a partir de aproximações da física óptica (do inglês, physical optics - PO) e com fases otimizadas através do método heurístico de busca global PSO (do inglês, particle swarm optimization). Em [71] é utilizado o FEKO para fins de comparação com os resultados obtidos a partir das análises iniciais aplicadas em RAs, tais como o método da teoria de redes de antenas e do campo na abertura.

Alguns dos métodos numéricos citados utilizam funções base conhecidas e de pesos a serem determinados, tais como o MoM e FEM. Estas funções podem ser de domínio completo ou de subdomínio. Funções de domínio completo são válidas ao longo da estrutura e geralmente possuem características senoidais. Já funções de subdomínio são válidas apenas dentro de determinada região do espaço e podem ser funções impulso e/ou pulso: quadrados, triangular, senoidal, etc.

\section{5}

\section{Síntese de redes refletoras}

As técnicas de análise eletromagnéticas apresentadas anteriormente devem ser combinadas com métodos de síntese de diagrama de irradiação, de forma a determinar a distribuição de fase nos elementos que compõem a estrutura refletora. Conforme abordado nas seções anteriores, grande parte dos trabalhos aplicam técnicas para se determinar apenas a fase (do inglês, phase only), que é analiticamente difícil de ser calculada quando diagramas conformados são desejados.

A combinação de técnica numérica e método de otimização de diagrama foi apresentada na ferramenta de [76], denominada GTOD. Neste trabalho o MoM foi aplicado em uma estrutura considerada periódica e combinado com uma ferramenta de otimização (denominada minimax) para modelar o padrão de irradiação.

Além do minimax, outras técnicas têm sido aplicadas em antenas 
nos últimos anos, tais como gradiente conjugado, algoritmo genético (GA), enxame de partículas (PSO) e abordagem de interseção (do inglês, Intersection Approach - IA) [6]. Entretanto, grande parte destes métodos apresentam limitações na síntese de diagramas modelados, pois algumas destas técnicas necessitam de um ponto de partida adequado para se obter ótima solução. Por exemplo, a função minimax é um método de busca local e sua convergência depende dos valores iniciais. Já o gradiente conjugado não é adequado para otimizações complexas não lineares. Enquanto isso, métodos globais, tais como PSO e GA, concentram-se na busca dentro de determinado espaço (exemplo: valores possíveis de fase para os elementos da RA estão na faixa de $0^{\circ}$ a $360^{\circ}$ ), entretanto apresentam lenta convergência para redes que possuam muitos elementos. Isto ocorre devido à necessidade de calcular o fitness para cada partícula/indivíduo que compõe o enxame/população. O IA é eficiente ao usar apenas a transformada rápida de Fourier (em inglês, fast Fourier transform FFT), mas sofre com o problema de armadilhas durante a execução [80], ou seja, converge para uma solução local que pode não ser uma ótima global.

\section{6}

\section{Erros na modelagem de RA}

Diversos fatores podem contribuir para erros no projeto de RAs, tais como:

i) modelamento da fonte: deve-se obter a representação adequada da antena utilizada para iluminar a RA, com o intuito de estimar com precisão o campo incidente em cada elemento. O campo pode ser obtido através de medidas em campo próximo e/ou distante;

ii) consideração da periodicidade local: as redes impressas reais não possuem configuração periódica, o que diverge com as aproximações realizadas em algumas técnicas de análise;

iii) escolha de funções base: deve-se optar por funções base adequadas para o formato da estrutura, de forma a aumentar o desempenho da análise dos parâmetros da antena e reduzir esforços computacionais;

iv) truncamento do plano de terra: a maioria dos trabalhos consideram redes infinitas durante a análise, entretanto o truncamento do plano de terra produz difração de borda, pois o campo irradiado pela fonte é transmitido da interface dielétrico-ar até a borda da RA. Dependendo da intensidade do campo difratado, que está relacionado às características do diagrama da fonte, além do formato do canto e das dimensões da rede, este efeito pode ser considerável, impactando no diagrama sintetizado. 


\section{7}

\section{Contribuições deste trabalho}

A partir da contextualização sobre redes refletoras, busca-se a solução de uma antena que possa ser embarcada em um nanossatélite de dimensão $20 \mathrm{~cm} \times 20 \mathrm{~cm}$. Supondo a operação da antena de downlink em qualquer frequência dentro da banda $\mathrm{S}$, têm-se os espaçamentos em termos de $\lambda_{0}$ para os limites da faixa descritos na Tabela 2.1. Sabendo-se que a distância selecionada deve garantir que não haja sobreposição de elementos impressos e permitir o maior controle possível do diagrama de irradiação, assume-se que os espaçamentos entre elementos adjacentes devem ficar na faixa de $0,35 \lambda_{0}$ a $0,6 \lambda_{0}$, assim, a análise fica restrita para estruturas quadradas compostas de 9 (RA $3 \times 3)$ a 49 elementos $($ RA $7 \times 7)$.

Tabela 2.1: Número de elementos $\times$ espaçamento.

\begin{tabular}{|c|c|c|c|}
\hline \multicolumn{2}{|c|}{$2 \mathrm{GHz}$} & \multicolumn{2}{c|}{$4 \mathrm{GHz}$} \\
\hline $\begin{array}{c}\mathrm{N}^{\circ} \text { elementos } \\
(\mathrm{RA})\end{array}$ & $\begin{array}{c}\text { Espaçamento } \\
\left(\lambda_{0}\right)\end{array}$ & $\begin{array}{c}\mathrm{N}^{\circ} \text { elementos } \\
(\mathrm{RA})\end{array}$ & $\begin{array}{c}\text { Espaçamento } \\
\left(\lambda_{0}\right)\end{array}$ \\
\hline $3 \times 3$ & 0,44 & $3 \times 3$ & 0,88 \\
\hline $4 \times 4$ & 0,33 & $4 \times 4$ & 0,66 \\
\hline $5 \times 5$ & 0,26 & $5 \times 5$ & 0,53 \\
\hline $6 \times 6$ & 0,22 & $6 \times 6$ & 0,44 \\
\hline $7 \times 7$ & 0,19 & $7 \times 7$ & 0,38 \\
\hline
\end{tabular}

Devido ao número reduzido de elementos, o foco deste trabalho é na modelagem de pequenas estruturas. Os trabalhos encontrados na literatura analisam redes refletoras da seguinte forma [6]:

1) Aplicam a formulação da função diádica de Green para a estrutura de microfita;

2) Consideram uma rede infinita de elementos idênticos e solução periódica usando a abordagem de Floquet;

3) Determinam o campo espalhado a partir de método numérico usando funções base apropriadas e diversos modos;

4) Extraem a curva de fase do elemento espalhador considerando periodicidade local;

5) Utilizam técnicas para a síntese do diagrama de irradiação para determinar a fase desejada e, consequentemente, a dimensão do elemento no caso de projeto com elementos de tamanhos variados.

A consideração de rede infinita é utilizada para a obtenção de apenas uma curva de fase para todos os elementos que compõe uma RA. Neste método de 
análise, considera-se uma onda incidindo com mesma amplitude sobre todos os elemento da rede. Entretanto, sabe-se que os efeitos do acoplamento mútuo entre os elementos da rede modificam a amplitude e fase do campo espalhado por cada metalização de uma rede finita. A intensidade de acoplamento depende da posição espacial de cada elemento irradiador na rede.

A abordagem de Floquet é utilizada para minimizar o esforço computacional, visto que as funções base utilizadas demandam um elevado número de modos para caracterizar o comportamento da corrente nas bordas do patch. Desta forma, a análise de redes com elevado número de elementos se torna inviável.

Os resultados obtidos nestes projetos apresentam excelente concordância com aqueles de ferramentas comerciais para a respectiva forma de análise aplicada a redes $15 \times 15$ ou superiores. Vale salientar que a excitação ocorre por uma fonte $\cos ^{q}$ bem diretiva. Assim, os elementos das bordas são pouco iluminados em comparação com os centrais da rede. Neste caso, os erros de fase introduzidos pelos elementos nas bordas são minimizados, tornando a contribuição pouco significativa sobre o padrão de irradiação da rede. Adicionalmente, os efeitos difrativos são minimizados quando a iluminação da borda for pouco intensa.

Deve-se dedicar atenção especial para redes com dimensões menores. Supondo RAs de dimensões de até $10 \times 10$, pode-se dizer que até $64 \%$ dos elementos estão localizados no centro, enquanto que, no mínimo, 36\% estão nas bordas. Entende-se que quanto menor a rede, maior deve ser a contribuição dos elementos próximos das periferias. Neste caso, deve-se investigar o erro introduzido por estes elementos.

As contribuições deste trabalho são:

1) A análise completa de redes finitas com poucos elementos baseandose no conceito de variação de fase a partir da dimensão do espalhador impresso. Para melhorar o desempenho computacional, aplica-se uma função base com condição de borda segmentada, demandando poucos modos para representar o comportamento impulsivo da densidade de corrente nas bordas das metalizações.

2) Extração de diferentes curvas de fase, sendo possível diferenciar o comportamento do campo espalhado pelos elementos alocados em diferentes posições espaciais na RA em função da variação das dimensões dos espalhadores, e, assim, reduzir o erro de fase;

3) O preenchimento da matriz $\bar{Z}$ do método dos momentos (MoM) demanda elevado tempo computacional para o cálculo dos coeficientes. Supondo uma rede finita de elementos idênticos, é possível verificar, devido à simetria 
e reciprocidade da estrutura, que muitos termos que compõem a matriz são iguais. Para fins de otimização do tempo, é necessário computar as integrais apenas uma vez para o preenchimento da matriz $\bar{Z}$;

4) Os efeitos de difração de borda em redes refletoras têm sido desprezados na obtenção da curva de fase, visto que a análise de Floquet não possibilita a inclusão deste fenômeno. Dependendo da característica de iluminação da rede, estes efeitos devem ser estudados para avaliar o impacto sobre as curvas de fase e o diagrama de irradiação sintetizado. 


\section{3 \\ Formulação da função diádica de Green para estruturas pla- nares}

Para um meio homogêneo, linear, isotrópico e sem fontes, as equações de Maxwell no domínio da frequência tornam-se,

$$
\begin{gathered}
\boldsymbol{\nabla} \times \mathbf{E}=-j w \mu \mathbf{H}, \\
\boldsymbol{\nabla} \times \mathbf{H}=j w \varepsilon \mathbf{E}, \\
\boldsymbol{\nabla} . \mathbf{H}=0
\end{gathered}
$$

$\mathrm{e}$

$$
\boldsymbol{\nabla} \cdot \mathbf{E}=0
$$

em que $\mu$ é a permeabilidade magnética, $\varepsilon$ é a permissividade elétrica, $w$ é a frequência angular, $\mathbf{H}$ e $\mathbf{E}$ são os vetores campo magnético e campo elétrico. Neste caso uma dependência $e^{j w t}$ é considerada, sendo $t$ a variável tempo.

A seguir é apresentada a dedução detalhada da função diádica de Green proposta em [73], sendo realizadas algumas correções nas equações de [73] devido a constatação de erros.

\section{1}

\section{Solução da equação de onda}

Tomando o rotacional de ambos os lados da (3-1),

$$
\nabla \times \nabla \times \mathbf{E}=-j w \mu \boldsymbol{\nabla} \times \mathbf{H}
$$

e aplicando a (3-2), tem-se que

$$
\nabla \times \nabla \times \mathbf{E}=w^{2} \mu \varepsilon \mathbf{E} .
$$

Dispondo da seguinte identidade,

$$
\nabla \times \nabla \times \zeta=\nabla[\nabla . \zeta]-\nabla^{2} \zeta
$$

aplicando a (3-4) e considerando que os meios sejam dielétricos perfeitos, ou seja não possuam condutividade associada $(\sigma=0)$, tal que a corrente de condução seja zero, tem-se que a equação de onda vetorial para o campo elétrico é dada por 


$$
\nabla^{2} \mathbf{E}+\varepsilon_{r} k_{0}^{2} \mathbf{E}=\mathbf{0}
$$

em que $\mu=\mu_{0}, \varepsilon_{e}=\varepsilon_{r} \varepsilon_{0}$, sendo $\mu_{0}$ e $\varepsilon_{0}$ a permeabilidade magnética e a permissividade elétrica no vácuo, respectivamente, e $\varepsilon_{r}$ é a permissividade relativa do meio de propagação.

Aplicando procedimento semelhante para a (3-2) e utilizando a (3-3), obtém-se a equação de onda vetorial para o campo magnético, descrita por

$$
\nabla^{2} \mathbf{H}+\varepsilon_{r} k_{0}^{2} \mathbf{H}=\mathbf{0} .
$$

Fazendo $\boldsymbol{\psi}$ iguais a $\mathbf{H}$ ou $\mathbf{E}$, e aplicando a seguinte consideração,

$$
\boldsymbol{\nabla}^{2} \boldsymbol{\psi}=\hat{x} \nabla^{2} \psi_{x}+\hat{y} \nabla^{2} \psi_{y}+\hat{z} \nabla^{2} \psi_{z}
$$

sendo

$$
\nabla^{2} \psi_{v}=\frac{\partial^{2} \psi_{v}}{\partial x^{2}}+\frac{\partial^{2} \psi_{v}}{\partial y^{2}}+\frac{\partial^{2} \psi_{v}}{\partial z^{2}} \operatorname{com} v=x, \text { you } z
$$

obtém-se

$$
\left(\frac{\partial^{2}}{\partial x^{2}}+\frac{\partial^{2}}{\partial y^{2}}+\frac{\partial^{2}}{\partial z^{2}}+\varepsilon_{r} k_{0}^{2}\right) \psi_{v}=0
$$

Aplicando normalização nas coordenadas espaciais, ou seja, $\xi=\frac{\bar{\xi}}{k_{0}}$, sendo $\xi$ uma direção arbitrária, então a (3-12) torna-se

$$
\left(\frac{\partial^{2}}{\partial \bar{x}^{2}}+\frac{\partial^{2}}{\partial \bar{y}^{2}}+\frac{\partial^{2}}{\partial \bar{z}^{2}}+\varepsilon_{r}\right) \psi_{v}=0
$$

A transformada dupla de Fourier nas coordenadas $x$ e $y$ é descrita como sendo

$$
\tilde{\psi}\left(k_{\bar{x}}, k_{\bar{y}}, \bar{z}\right)=\int_{-\infty}^{+\infty} \int_{-\infty}^{+\infty} \psi(\bar{x}, \bar{y}, \bar{z}) e^{-j k_{\bar{x}} \bar{x}} e^{-j k_{\bar{y}} \bar{y}} d \bar{x} d \bar{y}
$$

e sua inversa,

$$
\psi(\bar{x}, \bar{y}, \bar{z})=\frac{1}{(2 \pi)^{2}} \int_{-\infty}^{+\infty} \int_{-\infty}^{+\infty} \tilde{\psi}\left(k_{\bar{x}}, k_{\bar{y}}, \bar{z}\right) e^{j k_{\bar{x}} \bar{x}} e^{j k_{\bar{y}} \bar{y}} d k_{\bar{x}} d k_{\bar{y}}
$$

Assumem-se soluções na forma de ondas planas, descritas por $e^{ \pm j\left(k_{\bar{x}} \bar{x}+k_{\bar{y}} \bar{y}+k_{\bar{z}} \bar{z}\right)}$. Aplicando a transformada de Fourier, tem-se que $\frac{\partial}{\partial \bar{x}}= \pm j k_{\bar{x}}$ e $\frac{\partial}{\partial \bar{y}}= \pm j k_{\bar{y}}$, desta forma as derivadas de segunda ordem são representadas por $\frac{\partial^{2}}{\partial \bar{x}^{2}}=-k_{\bar{x}}^{2}, \frac{\partial^{2}}{\partial \bar{y}^{2}}=-k_{\bar{y}}^{2}$ e $\frac{\partial^{2}}{\partial \bar{y} \partial \bar{x}}=-k_{\bar{x}} k_{\bar{y}}$ no domínio espectral. A equação diferencial parcial torna-se

$$
\left(\frac{\partial^{2}}{\partial \bar{z}^{2}}-k_{\bar{z}}^{2}\right) \tilde{\psi}_{v}=0 \quad \text { com } v=x \text { ou } y
$$

sendo

$$
k_{\bar{z}}^{2}=k_{\bar{x}}^{2}+k_{\bar{y}}^{2}-\varepsilon_{r} .
$$

A solução para a equação diferencial ordinária é descrita da forma, 
Capítulo 3. Formulação da função diádica de Green para estruturas planares 39

$$
\tilde{\psi}_{v}=A e^{-k_{\bar{z}} \bar{z}}+B e^{k_{\bar{z}} \bar{z}} .
$$

A propagação de ondas eletromagnéticas requer que $k_{\bar{z}}$ seja um valor imaginário. Caso o meio possua perdas, então deve ser complexo. As equações (3-16) a (3-18) foram descritas neste formato para facilitar o equacionamento que satisfaça a condição de irradiação de Sommerfeld para um meio aberto, que consiste na atenuação dos campos eletromagnéticos à medida que se afastam da fonte. Maiores detalhes serão abordados no decorrer deste trabalho.

\section{2}

\section{Campos eletromagnéticos}

A partir das equações de Maxwell apresentadas em (3-1) e (3-2), e sabendo-se que

$$
\boldsymbol{\nabla} \times \boldsymbol{\zeta}=\hat{x}\left(\frac{\partial \zeta_{z}}{\partial y}-\frac{\partial \zeta_{y}}{\partial z}\right)+\hat{y}\left(\frac{\partial \zeta_{x}}{\partial z}-\frac{\partial \zeta_{z}}{\partial x}\right)+\hat{z}\left(\frac{\partial \zeta_{y}}{\partial x}-\frac{\partial \zeta_{x}}{\partial y}\right),
$$

$\log \mathrm{O}$

$$
\begin{aligned}
& \frac{\partial E_{z}}{\partial y}-\frac{\partial E_{y}}{\partial z}=-j w \mu H_{x} \\
& \frac{\partial H_{z}}{\partial y}-\frac{\partial H_{y}}{\partial z}=j w \varepsilon E_{x}
\end{aligned}
$$

para $\hat{x}$

$\mathrm{e}$

$$
\begin{aligned}
& \frac{\partial E_{x}}{\partial z}-\frac{\partial E_{z}}{\partial x}=-j w \mu H_{y} \\
& \frac{\partial H_{x}}{\partial z}-\frac{\partial H_{z}}{\partial x}=j w \varepsilon E_{y}
\end{aligned}
$$

para $\hat{y}$

$\mathrm{e}$

$$
\frac{\partial E_{y}}{\partial x}-\frac{\partial E_{x}}{\partial y}=-j w \mu H_{z}
$$

para $\hat{z}$, respectivamente.

$$
\frac{\partial H_{y}}{\partial x}-\frac{\partial H_{x}}{\partial y}=j w \varepsilon E_{z}
$$

\subsection{1}

\section{Relações dos campos eletromagnéticos Ey em função de Ex no domínio espacial}

Diferenciando ambos os lados da $(3-24)(\partial / \partial x)$,

$$
\frac{\partial^{2} E_{y}}{\partial x^{2}}-\frac{\partial^{2} E_{x}}{\partial y \partial x}=-j w \mu \frac{\partial H_{z}}{\partial x}
$$

e dispondo da (3-23), tem-se que 
Capítulo 3. Formulação da função diádica de Green para estruturas planares 40

$$
\frac{\partial^{2} E_{y}}{\partial x^{2}}-\frac{\partial^{2} E_{x}}{\partial y \partial x}=-j w \mu\left[\frac{\partial H_{x}}{\partial z}-j w \varepsilon E_{y}\right] .
$$

Organizando

em que

$$
\frac{\partial^{2} E_{y}}{\partial x^{2}}+\varepsilon_{r} k_{0}^{2} E_{y}=\frac{\partial^{2} E_{x}}{\partial y \partial x}-j w \mu_{0} \frac{\partial H_{x}}{\partial z}
$$

$$
k^{2}=w^{2} \varepsilon \mu=\varepsilon_{r} k_{0}^{2}
$$

foi considerado. Fazendo $x=\bar{x} / k_{0}$, a (3-28) torna-se

$$
k_{0}^{2} \frac{\partial^{2} E_{y}}{\partial \bar{x}^{2}}+\varepsilon_{r} k_{0}^{2} E_{y}=k_{0}^{2} \frac{\partial^{2} E_{x}}{\partial \bar{y} \partial \bar{x}}-j w \mu_{0} k_{0} \frac{\partial H_{x}}{\partial \bar{z}},
$$

dividindo por $k_{0}^{2}$ e organizando, tem-se

$$
\frac{\partial^{2} E_{y}}{\partial \bar{x}^{2}}+\varepsilon_{r} E_{y}=\frac{\partial^{2} E_{x}}{\partial \bar{y} \partial \bar{x}}-j \eta_{0} \frac{\partial H_{x}}{\partial \bar{z}},
$$

com $\eta_{0}=\sqrt{\mu_{0} / \varepsilon_{0}}$ correspondendo à impedância intrínseca do vácuo.

Diferenciando ambos os lados da (3-25) $(\partial / \partial x)$,

$$
\frac{\partial^{2} H_{y}}{\partial x^{2}}-\frac{\partial^{2} H_{x}}{\partial y \partial x}=j w \varepsilon \frac{\partial E_{z}}{\partial x}
$$

e dispondo da (3-22), tem-se que

$$
\frac{\partial^{2} H_{y}}{\partial x^{2}}-\frac{\partial^{2} H_{x}}{\partial y \partial x}=j w \varepsilon\left[\frac{\partial E_{x}}{\partial z}+j w \mu H_{y}\right] .
$$

Organizando, obtém-se

$$
\frac{\partial^{2} H_{y}}{\partial x^{2}}+\varepsilon_{r} k_{0}^{2} H_{y}=j w \varepsilon \frac{\partial E_{x}}{\partial z}+\frac{\partial^{2} H_{x}}{\partial y \partial x} .
$$

Aplicando a normalização,

$$
k_{0}^{2} \frac{\partial^{2} H_{y}}{\partial \bar{x}^{2}}+\varepsilon_{r} k_{0}^{2} H_{y}=j w \varepsilon k_{0} \frac{\partial E_{x}}{\partial \bar{z}}+k_{0}^{2} \frac{\partial^{2} H_{x}}{\partial \bar{y} \partial \bar{x}}
$$

e dividindo por $w \varepsilon_{0} k_{0}$, tem-se

$$
\eta_{0} \frac{\partial^{2} H_{y}}{\partial \bar{x}^{2}}+\varepsilon_{r} \eta_{0} H_{y}=j \varepsilon_{r} \frac{\partial E_{x}}{\partial \bar{z}}+\eta_{0} \frac{\partial^{2} H_{x}}{\partial \bar{y} \partial \bar{x}} .
$$

As equações (3-31) e (3-36) são reescritas na forma matricial como sendo,

$$
\left(\frac{\partial^{2}}{\partial \bar{x}^{2}}+\varepsilon_{r}\right)\left[\begin{array}{c}
E_{y} \\
\eta_{0} H_{y}
\end{array}\right]=\left[\begin{array}{cc}
\frac{\partial^{2}}{\partial \bar{y} \partial \bar{x}} & -j \frac{\partial}{\partial \bar{z}} \\
j \varepsilon_{r} \frac{\partial}{\partial \bar{z}} & \frac{\partial^{2}}{\partial \bar{y} \partial \bar{x}}
\end{array}\right]\left[\begin{array}{c}
E_{x} \\
\eta_{0} H_{x}
\end{array}\right] .
$$

\section{2 .2}

\section{Relações dos campos eletromagnéticos Ez em função de Ex no domínio espacial}

Diferenciando ambos os lados da (3-22) $(\partial / \partial x)$,

$$
\frac{\partial^{2} E_{x}}{\partial z \partial x}-\frac{\partial^{2} E_{z}}{\partial x^{2}}=-j w \mu \frac{\partial H_{y}}{\partial x}
$$


Capítulo 3. Formulação da função diádica de Green para estruturas planares 41

e dispondo da (3-25), tem-se que

$$
\frac{\partial^{2} E_{z}}{\partial x^{2}}=\frac{\partial^{2} E_{x}}{\partial z \partial x}+j w \mu\left[\frac{\partial H_{x}}{\partial y}+j w \varepsilon E_{z}\right] .
$$

Aplicando as considerações anteriores e normalizando, obtém-se

$$
k_{0}^{2} \frac{\partial^{2} E_{z}}{\partial \bar{x}^{2}}+\varepsilon_{r} k_{0}^{2} E_{z}=k_{0}^{2} \frac{\partial^{2} E_{x}}{\partial \bar{z} \partial \bar{x}}+j w \mu_{0} k_{0} \frac{\partial H_{x}}{\partial \bar{y}},
$$

dividindo por $k_{0}^{2}$ e organizando, então

$$
\frac{\partial^{2} E_{z}}{\partial \bar{x}^{2}}+\varepsilon_{r} E_{z}=\frac{\partial^{2} E_{x}}{\partial \bar{z} \partial \bar{x}}+j \eta_{0} \frac{\partial H_{x}}{\partial \bar{y}} .
$$

Diferenciando ambos os lados da $(3-23)(\partial / \partial x)$,

$$
\frac{\partial^{2} H_{x}}{\partial z \partial x}-\frac{\partial^{2} H_{z}}{\partial x^{2}}=j w \varepsilon \frac{\partial E_{y}}{\partial x}
$$

e dispondo da (3-24), tem-se que

$$
\frac{\partial^{2} H_{z}}{\partial x^{2}}=\frac{\partial^{2} H_{x}}{\partial z \partial x}-j w \varepsilon\left[\frac{\partial E_{x}}{\partial y}-j w \mu H_{z}\right],
$$

$\log 0$

$$
\frac{\partial^{2} H_{z}}{\partial x^{2}}+\varepsilon_{r} k_{0}^{2} H_{z}=-j w \varepsilon \frac{\partial E_{x}}{\partial y}+\frac{\partial^{2} H_{x}}{\partial z \partial x} .
$$

Aplicando a normalização,

$$
k_{0}^{2} \frac{\partial^{2} H_{z}}{\partial \bar{x}^{2}}+\varepsilon_{r} k_{0}^{2} H_{z}=k_{0}^{2} \frac{\partial^{2} H_{x}}{\partial \bar{z} \partial \bar{x}}-j w \varepsilon k_{0} \frac{\partial E_{x}}{\partial \bar{y}}
$$

e dividindo por $w \varepsilon_{0} k_{0}$, obtém-se

$$
\eta_{0} \frac{\partial^{2} H_{z}}{\partial \bar{x}^{2}}+\varepsilon_{r} \eta_{0} H_{z}=-j \varepsilon_{r} \frac{\partial E_{x}}{\partial \bar{y}}+\eta_{0} \frac{\partial^{2} H_{x}}{\partial \bar{z} \partial \bar{x}} .
$$

$\mathrm{Na}$ forma matricial, (3-41) e (3-46) são reescritas como sendo,

$$
\left(\frac{\partial^{2}}{\partial \bar{x}^{2}}+\varepsilon_{r}\right)\left[\begin{array}{c}
E_{z} \\
\eta_{0} H_{z}
\end{array}\right]=\left[\begin{array}{cc}
\frac{\partial^{2}}{\partial \bar{z} \partial \bar{x}} & j \frac{\partial}{\partial \bar{y}} \\
-j \varepsilon_{r} \frac{\partial}{\partial \bar{y}} & \frac{\partial^{2}}{\partial \bar{z} \partial \bar{x}}
\end{array}\right]\left[\begin{array}{c}
E_{x} \\
\eta_{0} H_{x}
\end{array}\right] .
$$

\subsection{3}

\section{Relações dos campos eletromagnéticos Ex em função de Ey no domínio espacial}

Diferenciando ambos os lados da (3-24) ( $\partial / \partial y)$,

$$
\frac{\partial^{2} E_{y}}{\partial x \partial y}-\frac{\partial^{2} E_{x}}{\partial y^{2}}=-j w \mu \frac{\partial H_{z}}{\partial y}
$$

e dispondo da (3-21), tem-se que

$\mathrm{Ou}$

$$
\frac{\partial^{2} E_{y}}{\partial x \partial y}-\frac{\partial^{2} E_{x}}{\partial y^{2}}=-j w \mu\left[\frac{\partial H_{y}}{\partial z}+j w \varepsilon E_{x}\right]
$$


Capítulo 3. Formulação da função diádica de Green para estruturas planares 42

$$
-\frac{\partial^{2} E_{x}}{\partial y^{2}}-\varepsilon_{r} k_{0}^{2} E_{x}=-\frac{\partial^{2} E_{y}}{\partial x \partial y}-j w \mu_{0} \frac{\partial H_{y}}{\partial z} .
$$

Normalizando,

$$
k_{0}^{2} \frac{\partial^{2} E_{x}}{\partial \bar{y}^{2}}+\varepsilon_{r} k_{0}^{2} E_{x}=k_{0}^{2} \frac{\partial^{2} E_{y}}{\partial \bar{x} \partial \bar{y}}+j w \mu_{0} k_{0} \frac{\partial H_{y}}{\partial \bar{z}}
$$

e dividindo por $k_{0}^{2}$, tem-se

$$
\frac{\partial^{2} E_{x}}{\partial \bar{y}^{2}}+\varepsilon_{r} E_{x}=\frac{\partial^{2} E_{y}}{\partial \bar{x} \partial \bar{y}}+j \eta_{0} \frac{\partial H_{y}}{\partial \bar{z}} .
$$

Diferenciando ambos os lados da (3-25) ( $\partial / \partial y)$,

$$
\frac{\partial^{2} H_{y}}{\partial x \partial y}-\frac{\partial^{2} H_{x}}{\partial y^{2}}=j w \varepsilon \frac{\partial E_{z}}{\partial y}
$$

e dispondo da (3-20), tem-se que

$$
\frac{\partial^{2} H_{y}}{\partial x \partial y}-\frac{\partial^{2} H_{x}}{\partial y^{2}}=j w \varepsilon\left[\frac{\partial E_{y}}{\partial z}-j w \mu H_{x}\right],
$$

$\log 0$

$$
\frac{-\partial^{2} H_{x}}{\partial y^{2}}-\varepsilon_{r} k_{0}^{2} H_{x}=-\frac{\partial^{2} H_{y}}{\partial x \partial y}+j w \varepsilon \frac{\partial E_{y}}{\partial z} .
$$

Aplicando a normalização,

$$
-k_{0}^{2} \frac{\partial^{2} H_{x}}{\partial \bar{y}^{2}}-\varepsilon_{r} k_{0}^{2} H_{x}=-k_{0}^{2} \frac{\partial^{2} H_{y}}{\partial \bar{x} \partial \bar{y}}+j w \varepsilon k_{0} \frac{\partial E_{y}}{\partial \bar{z}}
$$

e dividindo por $w \varepsilon_{0} k_{0}$, obtém-se

$$
-\eta_{0} \frac{\partial^{2} H_{x}}{\partial \bar{y}^{2}}-\varepsilon_{r} \eta_{0} H_{x}=-\eta_{0} \frac{\partial^{2} H_{y}}{\partial \bar{x} \partial \bar{y}}+j \varepsilon_{r} \frac{\partial E_{y}}{\partial \bar{z}} .
$$

Na forma matricial, (3-52) e (3-57) são reescritas como sendo,

$$
\left(\frac{\partial^{2}}{\partial \bar{y}^{2}}+\varepsilon_{r}\right)\left[\begin{array}{c}
E_{x} \\
-\eta_{0} H_{x}
\end{array}\right]=\left[\begin{array}{cc}
\frac{\partial^{2}}{\partial \bar{x} \partial \bar{y}} & -j \frac{\partial}{\partial \bar{z}} \\
j \varepsilon_{r} \frac{\partial}{\partial \bar{z}} & \frac{\partial^{2}}{\partial \bar{x} \partial \bar{y}}
\end{array}\right]\left[\begin{array}{c}
E_{y} \\
-\eta_{0} H_{y}
\end{array}\right] .
$$

\subsection{4}

\section{Relações dos campos eletromagnéticos no domínio espectral}

Fazendo uso da (3-14), as equações (3-37) e (3-58) ficam da seguinte forma no domínio espectral

$$
\begin{gathered}
\left(\varepsilon_{r}-k_{\bar{x}}^{2}\right)\left[\begin{array}{c}
\tilde{E}_{y} \\
\eta_{0} \tilde{H}_{y}
\end{array}\right]=\left[\begin{array}{cc}
-k_{\bar{x}} k_{\bar{y}} & -j \frac{\partial}{\partial \bar{z}} \\
j \varepsilon_{r} \frac{\partial}{\partial \bar{z}} & -k_{\bar{x}} k_{\bar{y}}
\end{array}\right]\left[\begin{array}{c}
\tilde{E}_{x} \\
\eta_{0} \tilde{H}_{x}
\end{array}\right] \\
\left(\varepsilon_{r}-k_{\bar{y}}^{2}\right)\left[\begin{array}{c}
\tilde{E}_{x} \\
-\eta_{0} \tilde{H}_{x}
\end{array}\right]=\left[\begin{array}{cc}
-k_{\bar{x}} k_{\bar{y}} & -j \frac{\partial}{\partial \bar{z}} \\
j \varepsilon_{r} \frac{\partial}{\partial \bar{z}} & -k_{\bar{x}} k_{\bar{y}}
\end{array}\right]\left[\begin{array}{c}
\tilde{E}_{y} \\
-\eta_{0} \tilde{H}_{y}
\end{array}\right] .
\end{gathered}
$$

Abrindo (3-59), 
Capítulo 3. Formulação da função diádica de Green para estruturas planares 43

e $(3-60)$

$$
\begin{gathered}
\left(\varepsilon_{r}-k_{\bar{x}}^{2}\right) \tilde{E}_{y}=-k_{\bar{x}} k_{\bar{y}} \tilde{E}_{x}-j \eta_{0} \frac{\partial \tilde{H}_{x}}{\partial \bar{z}} \\
\left(\varepsilon_{r}-k_{\bar{x}}^{2}\right)\left[\eta_{0} \tilde{H}_{y}\right]=j \varepsilon_{r} \frac{\partial \tilde{E}_{x}}{\partial \bar{z}}-k_{\bar{x}} k_{\bar{y}}\left[\eta_{0} \tilde{H}_{x}\right]
\end{gathered}
$$

$$
\begin{gathered}
\left(\varepsilon_{r}-k_{\bar{y}}^{2}\right) \tilde{E}_{x}=-k_{\bar{x}} k_{\bar{y}} \tilde{E}_{y}+j \eta_{0} \frac{\partial \tilde{H}_{y}}{\partial \bar{z}}, \\
\left(\varepsilon_{r}-k_{\bar{y}}^{2}\right)\left[-\eta_{0} \tilde{H}_{x}\right]=j \varepsilon_{r} \frac{\partial \tilde{E}_{y}}{\partial \bar{z}}+k_{\bar{x}} k_{\bar{y}}\left[\eta_{0} \tilde{H}_{y}\right],
\end{gathered}
$$

pode-se reescrever (3-61) e (3-63) na forma matricial,

$$
\bar{\partial} \bar{z}\left[\begin{array}{c}
-\eta_{0} \tilde{H}_{x} \\
\eta_{0} \tilde{H}_{y}
\end{array}\right]=-\left[\begin{array}{cc}
\varepsilon_{r}-k_{\bar{x}}^{2} & k_{\bar{x}} k_{\bar{y}} \\
k_{\bar{x}} k_{\bar{y}} & \varepsilon_{r}-k_{\bar{y}}^{2}
\end{array}\right]\left[\begin{array}{c}
j \tilde{E}_{y} \\
j \tilde{E}_{x}
\end{array}\right] .
$$

Fazendo

$$
\tilde{\mathbf{H}}=\eta_{0}\left[\begin{array}{c}
-\tilde{H}_{x} \\
\tilde{H}_{y}
\end{array}\right]
$$

$$
\tilde{\mathbf{E}}=j\left[\begin{array}{c}
\tilde{E}_{y} \\
\tilde{E}_{x}
\end{array}\right]
$$

e

$$
\overline{\tilde{y}}=\left[\begin{array}{cc}
\varepsilon_{r}-k_{\bar{x}}^{2} & k_{\bar{x}} k_{\bar{y}} \\
k_{\bar{x}} k_{\bar{y}} & \varepsilon_{r}-k_{\bar{y}}^{2}
\end{array}\right]
$$

tem-se

$$
\frac{\partial}{\partial \bar{z}} \tilde{\mathbf{H}}=-\overline{\tilde{y}} \tilde{\mathbf{E}}
$$

\subsection{5}

\section{Campos eletromagnéticos nas interfaces de uma camada dielétrica}

A partir da equação de onda no domínio espectral, conforme (3-16), e sua solução apresentada em (3-18), a seguinte representação do campo é obtida para uma camada dielétrica $i$, conforme a Figura 3.1,

$$
\tilde{\psi}_{v_{i-1}}=A_{i} e^{-k_{\bar{z}_{i}} \bar{z}_{i-1}}+B_{i} e^{k_{\bar{z}_{i}} \bar{z}_{i-1}}
$$

na interface inferior e

$$
\tilde{\psi}_{v_{i}}=A_{i} e^{-k_{\bar{z}_{i}} \bar{z}_{i}}+B_{i} e^{k_{\bar{z}_{i}} \bar{z}_{i}}
$$

na interface superior, respectivamente.

Diferenciando (3-18) em função de $\bar{z}(\partial / \partial \bar{z})$, tem-se

$$
\frac{\partial \tilde{\psi}_{v_{i-1}}}{\partial \bar{z}}=-k_{\bar{z}_{i}} A_{i} e^{-k_{\bar{z}_{i}} \bar{z}_{i-1}}+k_{\bar{z}_{i}} B_{i} e^{k_{\bar{z}_{i}} \bar{z}_{i-1}}
$$



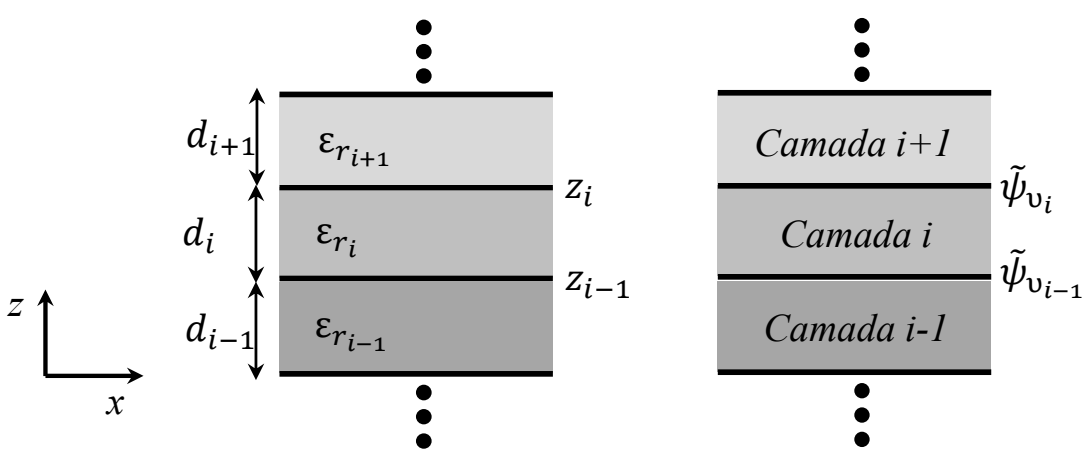

Figura 3.1: Estratificação de uma estrutura plana.

na interface inferior e na interface superior

$$
\frac{\partial \tilde{\psi}_{v_{i}}}{\partial \bar{z}}=-k_{\bar{z}_{i}} A_{i} e^{-k_{\bar{z}_{i}} \bar{z}_{i}}+k_{\bar{z}_{i}} B_{i} e^{k_{\bar{z}_{i}} \bar{z}_{i}}
$$

Na forma matricial, (3-70) e (3-71) ficam,

e (3-72) e (3-73),

$$
\left[\begin{array}{c}
\tilde{\psi}_{v_{i-1}} \\
\tilde{\psi}_{v_{i}}
\end{array}\right]=\left[\begin{array}{cc}
e^{-k_{\bar{z}_{i}} \bar{z}_{i-1}} & e^{k_{\bar{z}_{i}} \bar{z}_{i-1}} \\
e^{-k_{\bar{z}_{i}} \bar{z}_{i}} & e^{k_{\bar{z}_{i}} \bar{z}_{i}}
\end{array}\right]\left[\begin{array}{c}
A_{i} \\
B_{i}
\end{array}\right]
$$

$$
\frac{\partial}{\partial \bar{z}}\left[\begin{array}{c}
\tilde{\psi}_{v_{i-1}} \\
\tilde{\psi}_{v_{i}}
\end{array}\right]=\left[\begin{array}{cc}
-k_{\bar{z}_{i}} e^{-k_{\bar{z}_{i}} \bar{z}_{i-1}} & k_{\bar{z}_{i}} e^{k_{\bar{z}_{i}} \bar{z}_{i-1}} \\
-k_{\bar{z}_{i}} e^{-k_{\bar{z}_{i}} \bar{z}_{i}} & k_{\bar{z}_{i}} e^{k_{\bar{z}_{i}} \bar{z}_{i}}
\end{array}\right]\left[\begin{array}{c}
A_{i} \\
B_{i}
\end{array}\right] .
$$

Isolando o vetor que contém as constantes na (3-74) e substituindo na (3-75), tem-se

$$
\begin{aligned}
\frac{\partial}{\partial \bar{z}}\left[\begin{array}{c}
\tilde{\psi}_{v_{i-1}} \\
\tilde{\psi}_{v_{i}}
\end{array}\right]= \\
\quad\left[\begin{array}{cc}
-k_{\bar{z}_{i}} e^{-k_{\bar{z}_{i}} \bar{z}_{i-1}} & k_{\bar{z}_{i}} e^{k_{\bar{z}_{i}} \bar{z}_{i-1}} \\
-k_{\bar{z}_{i}} e^{-k_{\bar{z}_{i}} \bar{z}_{i}} & k_{\bar{z}_{i}} e^{k_{\bar{z}_{i}} \bar{z}_{i}}
\end{array}\right]\left[\begin{array}{cc}
e^{-k_{\bar{z}_{i}} \bar{z}_{i-1}} & e^{k_{\bar{z}_{i}} \bar{z}_{i-1}} \\
e^{-k_{\bar{z}_{i}} \bar{z}_{i}} & e^{k_{\bar{z}_{i}} \bar{z}_{i}}
\end{array}\right]^{-1}\left[\begin{array}{c}
\tilde{\psi}_{v_{i-1}} \\
\tilde{\psi}_{v_{i}}
\end{array}\right] .
\end{aligned}
$$

Resolvendo, obtém-se

em que

$$
\frac{\partial}{\partial \bar{z}}\left[\begin{array}{c}
\tilde{\psi}_{v_{i-1}} \\
\tilde{\psi}_{v_{i}}
\end{array}\right]=k_{\bar{z}_{i}}^{2} \bar{\Gamma}_{i}\left[\begin{array}{c}
\tilde{\psi}_{v_{i-1}} \\
\tilde{\psi}_{v_{i}}
\end{array}\right],
$$

com

$$
\bar{\Gamma}_{i}=\left[\begin{array}{cc}
-\gamma_{i} & \alpha_{i} \\
-\alpha_{i} & \gamma_{i}
\end{array}\right]
$$

$$
\gamma_{i}=\frac{1}{k_{\bar{z}_{i}} \tanh \left(k_{\bar{z}_{i}} \bar{d}_{i}\right)}
$$

$\mathrm{e}$ 
Capítulo 3. Formulação da função diádica de Green para estruturas planares 45

$$
\alpha_{i}=\frac{1}{k_{\bar{z}_{i}} \operatorname{senh}\left(k_{\bar{z}_{i}} \bar{d}_{i}\right)} .
$$

A multiplicação das expressões anteriores por $\left(k_{\bar{z}_{i}} / k_{\bar{z}_{i}}\right)$ facilita na representação futura da solução das matrizes inversas.

\subsection{6}

\section{Dedução da função de Green para estrutura com múltiplas camadas e metalização}

Fazendo-se uso de (3-77) a (3-80), pode-se escrever da seguinte forma a relação dos campos eletromagnéticos para uma camada $i$,

$$
\frac{\partial}{\partial \bar{z}}\left[\begin{array}{c}
-\eta_{0} \tilde{H}_{x_{i-1}} \\
\eta_{0} \tilde{H}_{y_{i-1}} \\
-\eta_{0} \tilde{H}_{x_{i}} \\
\eta_{0} \tilde{H}_{y_{i}}
\end{array}\right]=k_{\bar{z}_{i}}^{2}\left[\begin{array}{cccc}
-\gamma_{i} & 0 & \alpha_{i} & 0 \\
0 & -\gamma_{i} & 0 & \alpha_{i} \\
-\alpha_{i} & 0 & \gamma_{i} & 0 \\
0 & -\alpha_{i} & 0 & \gamma_{i}
\end{array}\right]\left[\begin{array}{c}
-\eta_{0} \tilde{H}_{x_{i-1}} \\
\eta_{0} \tilde{H}_{y_{i-1}} \\
-\eta_{0} \tilde{H}_{x_{i}} \\
\eta_{0} \tilde{H}_{y_{i}}
\end{array}\right]
$$

mas de (3-65) a (3-69), tem-se que

$$
\begin{aligned}
\frac{\partial}{\partial \bar{z}}\left[\begin{array}{c}
-\eta_{0} \tilde{H}_{x_{i-1}} \\
\eta_{0} \tilde{H}_{y_{i-1}} \\
-\eta_{0} \tilde{H}_{x_{i}} \\
\eta_{0} \tilde{H}_{y_{i}}
\end{array}\right]= \\
-\left[\begin{array}{cccc}
\varepsilon_{r_{i}}-k_{\bar{x}}^{2} & k_{\bar{x}} k_{\bar{y}} & 0 & 0 \\
k_{\bar{x}} k_{\bar{y}} & \varepsilon_{r_{i}}-k_{\bar{y}}^{2} & 0 & 0 \\
0 & 0 & \varepsilon_{r_{i}}-k_{\bar{x}}^{2} & k_{\bar{x}} k_{\bar{y}} \\
0 & 0 & k_{\bar{x}} k_{\bar{y}} & \varepsilon_{r_{i}}-k_{\bar{y}}^{2}
\end{array}\right]\left[\begin{array}{c}
j \tilde{E}_{y_{i-1}} \\
j \tilde{E}_{x_{i-1}} \\
j \tilde{E}_{y_{i}} \\
j \tilde{E}_{x_{i}}
\end{array}\right] .
\end{aligned}
$$

Substituindo (3-81) na (3-82), obtém-se

$$
\begin{aligned}
& k_{\bar{z}_{i}}^{2}\left[\begin{array}{cccc}
-\gamma_{i} & 0 & \alpha_{i} & 0 \\
0 & -\gamma_{i} & 0 & \alpha_{i} \\
-\alpha_{i} & 0 & \gamma_{i} & 0 \\
0 & -\alpha_{i} & 0 & \gamma_{i}
\end{array}\right]\left[\begin{array}{c}
-\eta_{0} \tilde{H}_{x_{i-1}} \\
\eta_{0} \tilde{H}_{y_{i-1}} \\
-\eta_{0} \tilde{H}_{x_{i}} \\
\eta_{0} \tilde{H}_{y_{i}}
\end{array}\right]= \\
&-\left[\begin{array}{cccc}
\varepsilon_{r_{i}}-k_{\bar{x}}^{2} & k_{\bar{x}} k_{\bar{y}} & 0 & 0 \\
k_{\bar{x}} k_{\bar{y}} & \varepsilon_{r_{i}}-k_{\bar{y}}^{2} & 0 & 0 \\
0 & 0 & \varepsilon_{r_{i}}-k_{\bar{x}}^{2} & k_{\bar{x}} k_{\bar{y}} \\
0 & 0 & k_{\bar{x}} k_{\bar{y}} & \varepsilon_{r_{i}}-k_{\bar{y}}^{2}
\end{array}\right]\left[\begin{array}{c}
j \tilde{E}_{y_{i-1}} \\
j \tilde{E}_{x_{i-1}} \\
j \tilde{E}_{y_{i}} \\
j \tilde{E}_{x_{i}}
\end{array}\right] .
\end{aligned}
$$

Vale salientar que o índice $i$ é suprimido de $k_{\bar{y}}$ e $k_{\bar{x}}$, pois as variações de fase dos campos eletromagnéticos de dois meios distintos devem ser satisfeitas na interface localizada no plano $x-y$. Neste caso, considera-se meio inomogêneo apenas em $z$. 
Organizando (3-83), tem-se

$$
\begin{array}{r}
{\left[\begin{array}{c}
-\eta_{0} \tilde{H}_{x_{i-1}} \\
\eta_{0} \tilde{H}_{y_{i-1}} \\
-\eta_{0} \tilde{H}_{x_{i}} \\
\eta_{0} \tilde{H}_{y_{i}}
\end{array}\right]=-\left[\begin{array}{cccc}
-\gamma_{i} & 0 & \alpha_{i} & 0 \\
0 & -\gamma_{i} & 0 & \alpha_{i} \\
-\alpha_{i} & 0 & \gamma_{i} & 0 \\
0 & -\alpha_{i} & 0 & \gamma_{i}
\end{array}\right]} \\
\times\left[\begin{array}{cccc}
\varepsilon_{r_{i}}-k_{\bar{x}}^{2} & k_{\bar{x}} k_{\bar{y}} & 0 & 0 \\
k_{\bar{x}} k_{\bar{y}} & \varepsilon_{r_{i}}-k_{\bar{y}}^{2} & 0 & 0 \\
0 & 0 & \varepsilon_{r_{i}}-k_{\bar{x}}^{2} & k_{\bar{x}} k_{\bar{y}} \\
0 & 0 & k_{\bar{x}} k_{\bar{y}} & \varepsilon_{r_{i}}-k_{\bar{y}}^{2}
\end{array}\right]\left[\begin{array}{c}
j \tilde{E}_{y_{i-1}} \\
j \tilde{E}_{x_{i-1}} \\
j \tilde{E}_{y_{i}} \\
j \tilde{E}_{x_{i}}
\end{array}\right],
\end{array}
$$

que pode ser reescrita como sendo,

$$
\left[\begin{array}{c}
\tilde{\mathbf{H}}_{i-1} \\
\tilde{\mathbf{H}}_{i}
\end{array}\right]=-\left[\bar{\Gamma}_{i} \otimes \overline{\tilde{y}}\right]\left[\begin{array}{c}
\tilde{\mathbf{E}}_{i-1} \\
\tilde{\mathbf{E}}_{i}
\end{array}\right],
$$

onde $\otimes$ representa o produto matricial de Kronecker,

$$
\begin{aligned}
\tilde{\mathbf{H}}_{i-1} & =\left[\begin{array}{c}
-\eta_{0} \tilde{H}_{x_{i-1}} \\
\eta_{0} \tilde{H}_{y_{i-1}}
\end{array}\right], \\
\tilde{\mathbf{E}}_{i-1} & =\left[\begin{array}{c}
j \tilde{E}_{y_{i-1}} \\
j \tilde{E}_{x_{i-1}}
\end{array}\right], \\
\tilde{\mathbf{H}}_{i} & =\left[\begin{array}{c}
-\eta_{0} \tilde{H}_{x_{i}} \\
\eta_{0} \tilde{H}_{y_{i}}
\end{array}\right] \\
\tilde{\mathbf{E}}_{i} & =\left[\begin{array}{c}
j \tilde{E}_{y_{i}} \\
j \tilde{E}_{x_{i}}
\end{array}\right],
\end{aligned}
$$

sendo $\bar{\Gamma}_{i}$ e $\overline{\tilde{y}}$ conhecidos de (3-78) e (3-68), com $\varepsilon_{r_{i}}=\varepsilon_{r}$.

Abrindo e organizando a (3-83), tem-se o seguinte sistema de equações,

$$
\begin{gathered}
\alpha_{i} k_{\bar{z}_{i}}^{2}\left[-\eta_{0} \tilde{H}_{x_{i}}\right]=\left(\varepsilon_{r_{i}}-k_{\bar{x}}^{2}\right)\left[-j \tilde{E}_{y_{i-1}}\right]+k_{\bar{x}} k_{\bar{y}}\left[-j \tilde{E}_{x_{i-1}}\right]+\gamma_{i} k_{\bar{z}_{i}}^{2}\left[-\eta_{0} \tilde{H}_{x_{i-1}}\right] \\
\alpha_{i} k_{\bar{z}_{i}}^{2}\left[\eta_{0} \tilde{H}_{y_{i}}\right]=k_{\bar{x}} k_{\bar{y}}\left[-j \tilde{E}_{y_{i-1}}\right]+\left(\varepsilon_{r_{i}}-k_{\bar{y}}^{2}\right)\left[-j \tilde{E}_{x_{i-1}}\right]+\gamma_{i} k_{\bar{z}_{i}}^{2}\left[\eta_{0} \tilde{H}_{y_{i-1}}\right] \\
\gamma_{i} k_{\bar{z}_{i}}^{2}\left[-\eta_{0} \tilde{H}_{x_{i}}\right]-\left(\varepsilon_{r_{i}}-k_{\bar{x}}^{2}\right)\left[-j \tilde{E}_{y_{i}}\right]-k_{\bar{x}} k_{\bar{y}}\left[-j \tilde{E}_{x_{i}}\right]=\alpha_{i} k_{\bar{z}_{i}}^{2}\left[-\eta_{0} \tilde{H}_{x_{i-1}}\right]
\end{gathered}
$$

e

$$
\gamma_{i} k_{\bar{z}_{i}}^{2}\left[\eta_{0} \tilde{H}_{y_{i}}\right]-k_{\bar{x}} k_{\bar{y}}\left[-j \tilde{E}_{y_{i}}\right]-\left(\varepsilon_{r_{i}}-k_{\bar{y}}^{2}\right)\left[-j \tilde{E}_{x_{i}}\right]=\alpha_{i} k_{\bar{z}_{i}}^{2}\left[\eta_{0} \tilde{H}_{y_{i-1}}\right]
$$


Na forma matricial,

$$
\begin{gathered}
{\left[\begin{array}{cccc}
0 & 0 & \left(\alpha_{i} k_{\bar{z}_{i}}^{2}\right) & 0 \\
0 & 0 & 0 & \left(\alpha_{i} k_{\bar{z}_{i}}^{2}\right) \\
-\left(\varepsilon_{r_{i}}-k_{\bar{x}}^{2}\right) & -\left(k_{\bar{x}} k_{\bar{y}}\right) & \left(\gamma_{i} k_{\bar{z}_{i}}^{2}\right) & 0 \\
-\left(k_{\bar{x}} k_{\bar{y}}\right) & -\left(\varepsilon_{r_{i}}-k_{\bar{y}}^{2}\right) & 0 & \left(\gamma_{i} k_{\bar{z}_{i}}^{2}\right)
\end{array}\right]\left[\begin{array}{c}
-j \tilde{E}_{y_{i}} \\
-j \tilde{E}_{x_{i}} \\
-\eta_{0} \tilde{H}_{x_{i}} \\
\eta_{0} \tilde{H}_{y_{i}}
\end{array}\right]=} \\
{\left[\begin{array}{cccc}
\left(\varepsilon_{r_{i}}-k_{\bar{x}}^{2}\right) & \left(k_{\bar{x}} k_{\bar{y}}\right) & \left(\gamma_{i} k_{\bar{z}_{i}}^{2}\right) & 0 \\
\left(k_{\bar{x}} k_{\bar{y}}\right) & \left(\varepsilon_{r_{i}}-k_{\bar{y}}^{2}\right) & 0 & \left(\gamma_{i} k_{\bar{z}_{i}}^{2}\right) \\
0 & 0 & \left(\alpha_{i} k_{\bar{z}_{i}}^{2}\right) & 0 \\
0 & 0 & 0 & \left(\alpha_{i} k_{\bar{z}_{i}}^{2}\right)
\end{array}\right]\left[\begin{array}{c}
-j \tilde{E}_{y_{i-1}} \\
-j \tilde{E}_{x_{i-1}} \\
-\eta_{0} \tilde{H}_{x_{i-1}} \\
\eta_{0} \tilde{H}_{y_{i-1}}
\end{array}\right]}
\end{gathered}
$$

Considerando que $k_{\bar{z}_{i}}^{2}=k_{\bar{x}}^{2}+k_{\bar{y}}^{2}-\varepsilon_{r_{i}}$, e organizando (3-94), tem-se

$$
\begin{aligned}
& {\left[\begin{array}{c}
j \tilde{E}_{y_{i}} \\
j \tilde{E}_{x_{i}} \\
-\eta_{0} \tilde{H}_{x_{i}} \\
\eta_{0} \tilde{H}_{y_{i}}
\end{array}\right]=\left[\begin{array}{cccc}
\cosh \left(k_{\bar{z}_{i}} \bar{d}_{i}\right) & 0 & -\frac{\left(k_{\bar{y}}^{2}-\varepsilon_{r_{i}}\right)}{\alpha_{i} \varepsilon_{r_{i}} k_{\bar{z}_{i}}^{2}} & -\frac{k_{\bar{x}} k_{\bar{y}}}{\alpha_{i} r_{r_{i}} k_{\bar{z}_{i}}^{2}} \\
0 & \cosh \left(k_{\bar{z}_{i}} \bar{d}_{i}\right) & -\frac{k_{\bar{x}} k_{\bar{y}}}{\alpha_{i} \varepsilon_{r_{i}} k_{\bar{z}_{i}}^{2}} & -\frac{\left(k_{\bar{x}}^{2}-\varepsilon_{r_{i}}\right)}{\alpha_{i} \varepsilon_{r_{i}} k_{\bar{z}_{i}}} \\
-\frac{\left(\varepsilon_{r_{i}}-k_{\bar{x}}^{2}\right)}{\alpha_{i} k_{\bar{z}_{i}}^{2}} & -\frac{k_{\bar{x}} k_{\bar{y}}}{\alpha_{i} k_{\bar{z}_{i}}^{2}} & \cosh \left(k_{\bar{z}_{i}} \bar{d}_{i}\right) & 0 \\
-\frac{k_{\bar{x}} k_{\bar{y}}}{\alpha_{i} k_{\bar{z}_{i}}^{2}} & -\frac{\left(\varepsilon_{r_{i}}-k_{\bar{y}}^{2}\right)}{\alpha_{i} k_{\bar{z}_{i}}^{2}} & 0 & \cosh \left(k_{\bar{z}_{i}} \bar{d}_{i}\right)
\end{array}\right] } \\
& \times\left[\begin{array}{c}
j \tilde{E}_{y_{i-1}} \\
j \tilde{E}_{x_{i-1}} \\
-\eta_{0} \tilde{H}_{x_{i-1}} \\
\eta_{0} \tilde{H}_{y_{i-1}}
\end{array}\right]
\end{aligned}
$$

que pode ser reescrita da seguinte forma,

$$
\tilde{\mathbf{F}}_{i}=\overline{\tilde{K}}_{i} \tilde{\mathbf{F}}_{i-1},
$$

sendo

e

$$
\begin{gathered}
\tilde{\mathbf{F}}_{i}=\left[\begin{array}{c}
\tilde{\mathbf{E}}_{i} \\
\tilde{\mathbf{H}}_{i}
\end{array}\right], \\
\tilde{\mathbf{F}}_{i-1}=\left[\begin{array}{c}
\tilde{\mathbf{E}}_{i-1} \\
\tilde{\mathbf{H}}_{i-1}
\end{array}\right]
\end{gathered}
$$

$$
\overline{\tilde{K}}_{i}=\left[\begin{array}{cc}
\overline{\tilde{V}}_{i} & -\overline{\tilde{Z}}_{i} \\
-\overline{\tilde{Y}}_{i} & \overline{\tilde{B}}_{i}
\end{array}\right],
$$

em que $\overline{\tilde{K}}_{i}$ possui dimensão $4 \times 4$ e é conhecida como matriz híbrida da camada $i$, cujas submatrizes $2 \times 2$ que a compõe descritas por

$$
\overline{\tilde{V}}_{i}=\overline{\tilde{B}}_{i}=\left[\begin{array}{cc}
\cosh \left(k_{\bar{z}_{i}} \bar{d}_{i}\right) & 0 \\
0 & \cosh \left(k_{\bar{z}_{i}} \bar{d}_{i}\right)
\end{array}\right],
$$


e

$$
\overline{\tilde{Z}}_{i}=\frac{1}{\alpha_{i} \varepsilon_{r_{i}} k_{\bar{z}_{i}}^{2}}\left[\begin{array}{cc}
\left(k_{\bar{y}}^{2}-\varepsilon_{r_{i}}\right) & k_{\bar{x}} k_{\bar{y}} \\
k_{\bar{x}} k_{\bar{y}} & \left(k_{\bar{x}}^{2}-\varepsilon_{r_{i}}\right)
\end{array}\right]
$$

$$
\overline{\tilde{Y}}_{i}=\frac{1}{\alpha_{i} k_{\bar{z}_{i}}^{2}}\left[\begin{array}{cc}
\left(\varepsilon_{r_{i}}-k_{\bar{x}}^{2}\right) & k_{\bar{x}} k_{\bar{y}} \\
k_{\bar{x}} k_{\bar{y}} & \left(\varepsilon_{r_{i}}-k_{\bar{y}}^{2}\right)
\end{array}\right] .
$$

A equação (3-96) combina todas as componentes dos campos tangenciais em ambos os lados de uma camada arbitrária $i$. Na Figura 3.2 pode ser visualizada a respectiva relação, bem como seu modelo circuital.
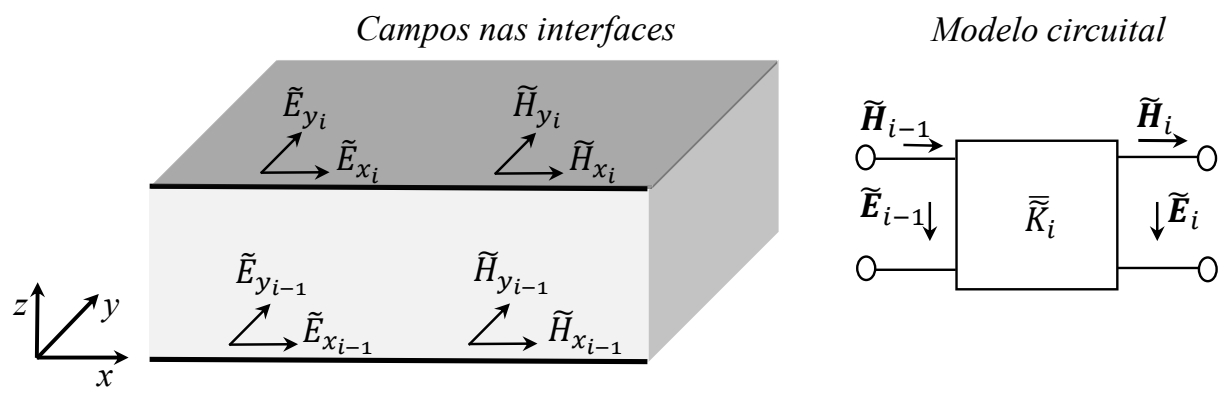

Figura 3.2: Campos eletromagnéticos tangenciais e modelo circuital.

Caso a interface inferior esteja aberta, então $\tilde{H}_{x_{i-1}}, \tilde{H}_{y_{i-1}}, \tilde{E}_{x_{i-1}}$ e $\tilde{E}_{y_{i-1}}$ devem desvanecer para satisfazer as condições de irradiação de Sommerfeld. Usando (3-84) após a observação apresentada, tem-se

$$
\begin{gathered}
\left.\alpha_{i}\left(\varepsilon_{r_{i}}-k_{\bar{x}}^{2}\right)\left[-j \tilde{E}_{y_{i}}\right]+\alpha_{i} k_{\bar{x}} k_{\bar{y}} \tilde{[}-j \tilde{E}_{x_{i}}\right]=0, \\
\alpha_{i} k_{\bar{x}} k_{\bar{y}}\left[-j \tilde{E}_{y_{i}}\right]+\alpha_{i}\left(\varepsilon_{r_{i}}-k_{\bar{y}}^{2}\right)\left[-j \tilde{E}_{x_{i}}\right]=0, \\
{\left[-\eta_{0} \tilde{H}_{x_{i}}\right]=\gamma_{i}\left(\varepsilon_{r_{i}}-k_{\bar{x}}^{2}\right)\left[-j \tilde{E}_{y_{i}}\right]+\gamma_{i} k_{\bar{x}} k_{\bar{y}}\left[-j \tilde{E}_{x_{i}}\right]}
\end{gathered}
$$

e

$$
\left[\eta_{0} \tilde{H}_{y_{i}}\right]=\gamma_{i} k_{\bar{x}} k_{\bar{y}}\left[-j \tilde{E}_{y_{i}}\right]+\gamma_{i}\left(\varepsilon_{r_{i}}-k_{\bar{y}}^{2}\right)\left[-j \tilde{E}_{x_{i}}\right]
$$

Para satisfazer (3-103) e (3-104), $\alpha_{i}$ deve ser zero. Para isto, a função $\operatorname{senh}\left( \pm k_{\bar{z}_{i}} \bar{d}_{i}\right) \rightarrow \pm \infty$, o que ocorre apenas para argumentos grandes. Caso não fosse assumido $k_{\bar{z}_{i}}$ imaginário na (3-16), então neste ponto a consideração de um argumento imaginário deveria ser utilizada para satisfazer a respectiva condição, exigindo que as equações ao longo deste documento fossem atualizadas. Por este motivo, (3-16) a (3-18) foram descritas no respectivo formato. 
A expressão $A_{i} e^{ \pm k_{\bar{z}_{i}} \bar{z}_{i}}$ descreve ondas se propagando para a direção $-z$ $\mathrm{ou}+z$, respectivamente. Desta forma, a propagação deve ocorrer na direção $-z$ quando uma camada estiver aberta em sua região inferior, resultando no termo de fase $+k_{\bar{z}_{i}} \bar{z}_{i}$. A função $\operatorname{senh}\left(k_{\bar{z}_{i}} \bar{d}_{i}\right)$, com $\bar{d}_{i} \rightarrow-\infty$, resulta em $\gamma_{i}=\frac{-1}{k_{\bar{z}_{i}}}$, logo (3-105) e (3-106) são descritas por,

$$
\tilde{\mathbf{H}}_{0}=-\overline{\tilde{Y}}_{0} \tilde{\mathbf{E}}_{0}
$$

sendo

$$
\overline{\tilde{Y}}_{0}=-\frac{1}{k_{\bar{z}_{0}}} \overline{\tilde{y}}_{0}
$$

em que $i=0$ é aplicado para denotar a interface inferior.

Já para a região superior aberta, $\bar{d}_{i} \rightarrow+\infty$ e $\gamma_{i}=\frac{1}{k_{\bar{z}}}$. Logo, a partir da (3-105) e (3-106), obtém-se,

$$
\tilde{\mathbf{H}}_{n}=\overline{\tilde{Y}}_{n+1} \tilde{\mathbf{E}}_{n}
$$

sendo

$$
\overline{\tilde{Y}}_{n+1}=-\frac{1}{k_{\bar{z}_{n+1}}} \overline{\tilde{y}}_{n+1},
$$

em que $i=n$ é aplicado para denotar a interface superior.

Supondo a existência de metalização sem perdas (do inglês, Perfect Electric Conductor - PEC) em uma interface $m$, as seguintes relações devem ser satisfeitas,

$$
\tilde{\mathbf{E}}_{m}^{+}=\tilde{\mathbf{E}}_{m}^{-}
$$

para as componentes tangenciais do campo elétrico, e

$$
\tilde{\mathbf{H}}_{m}^{+}-\tilde{\mathbf{H}}_{m}^{-}=\tilde{\mathbf{J}}^{(s)}=-\eta_{0}\left[\begin{array}{c}
\tilde{J}_{y} \\
\tilde{J}_{x}
\end{array}\right]
$$

para o campo magnético. Nota-se que a descontinuidade no campo magnético resulta em uma distribuição de corrente superficial sobre a superfície metálica.

Fazendo uso da (3-96) e assumindo um meio estratificado com $M$ camadas de espessura limitada (fechada) entre metalizações, então tem-se que

$$
\begin{gathered}
\tilde{\mathbf{F}}_{1}=\overline{\tilde{K}}_{1} \tilde{\mathbf{F}}_{0}, \\
\tilde{\mathbf{F}}_{2}=\overline{\tilde{K}}_{2} \tilde{\mathbf{F}}_{1}, \\
\tilde{\mathbf{F}}_{3}=\overline{\tilde{K}}_{3} \tilde{\mathbf{F}}_{2}, \\
\tilde{\mathbf{F}}_{g}=\overline{\tilde{K}}_{g} \tilde{\mathbf{F}}_{g-1}
\end{gathered}
$$




$$
\tilde{\mathbf{F}}_{M}=\overline{\tilde{K}}_{M} \tilde{\mathbf{F}}_{M-1}
$$

Organizando na forma de entrada e saída, ou seja, substituindo a (3-113) na (3-114), e assim sucessivamente até a (3-117), tem-se

$$
\tilde{\mathbf{F}}_{M}=\overline{\tilde{K}}_{M} \ldots \overline{\tilde{K}}_{g} \ldots \overline{\tilde{K}}_{3} \overline{\tilde{K}}_{2} \overline{\tilde{K}}_{1} \tilde{\mathbf{F}}_{0}
$$

Logo, define-se

$$
\overline{\tilde{K}}_{e q}=\prod_{i=1}^{M} \overline{\tilde{K}}_{i}
$$

em que $\overline{\tilde{K}}_{e q}$ representa um substrato equivalente de $M$ camadas estratificadas.

Supondo a existência de uma metalização em uma interface $m$, com $M_{1}$ camadas abaixo e $M_{2}$ camadas acima do PEC (regiões com dimensões limitadas), tem-se

$\mathrm{e}$

$$
\begin{gathered}
\overline{\tilde{K}}_{b}=\prod_{i=1}^{m} \overline{\tilde{K}}_{i} \quad \text { com } m=M_{1} \\
\overline{\tilde{K}}_{a}=\prod_{i=m+1}^{n} \overline{\tilde{K}}_{i} \quad \text { com } n=M_{2},
\end{gathered}
$$

com a seguinte relação entre os campos eletromagnéticos,

$$
\begin{gathered}
\tilde{\mathbf{F}}_{m}^{-}=\overline{\tilde{K}}_{b} \tilde{\mathbf{F}}_{0} \\
\tilde{\mathbf{F}}_{n}=\overline{\tilde{K}}_{a} \tilde{\mathbf{F}}_{m}^{+},
\end{gathered}
$$

conforme ilustrado na Figura 3.3.

Por fim, para fins de obtenção da função de Green para uma rede refletora formada por camadas dielétricas e uma metalização, assume-se a existência do plano de terra na interface inferior e que a estrutura esteja aberta na região superior (meio denotado por $n+1$ ), conforme esquemático apresentado na Figura 3.4.

Assim, fazendo uso da (3-96) a (3-99), e aplicando na (3-122), tem-se

$$
\left[\begin{array}{c}
\tilde{\mathbf{E}}_{m}^{-} \\
\tilde{\mathbf{H}}_{m}^{-}
\end{array}\right]=\left[\begin{array}{cc}
\overline{\tilde{V}}_{b} & -\overline{\tilde{Z}}_{b} \\
-\tilde{\tilde{Y}}_{b} & \overline{\tilde{B}}_{b}
\end{array}\right]\left[\begin{array}{c}
\tilde{\mathbf{E}}_{0} \\
\tilde{\mathbf{H}}_{0}
\end{array}\right] .
$$

A condição de contorno no plano de terra para o campo elétrico implica que $\tilde{\mathbf{E}}_{0}=\mathbf{0}$. Logo, a (3-124) torna-se

$$
\tilde{\mathbf{E}}_{m}^{-}=-\overline{\tilde{Z}}_{b} \tilde{\mathbf{H}}_{0}
$$

$\mathrm{e}$

$$
\tilde{\mathbf{H}}_{m}^{-}=\overline{\tilde{B}}_{b} \tilde{\mathbf{H}}_{0} .
$$

Isolando $\tilde{\mathbf{H}}_{0}$ na (3-125) e substituindo na (3-126), tem-se

$$
\tilde{\mathbf{H}}_{m}^{-}=-\overline{\tilde{Y}}^{(b)} \tilde{\mathbf{E}}_{m}^{-}
$$




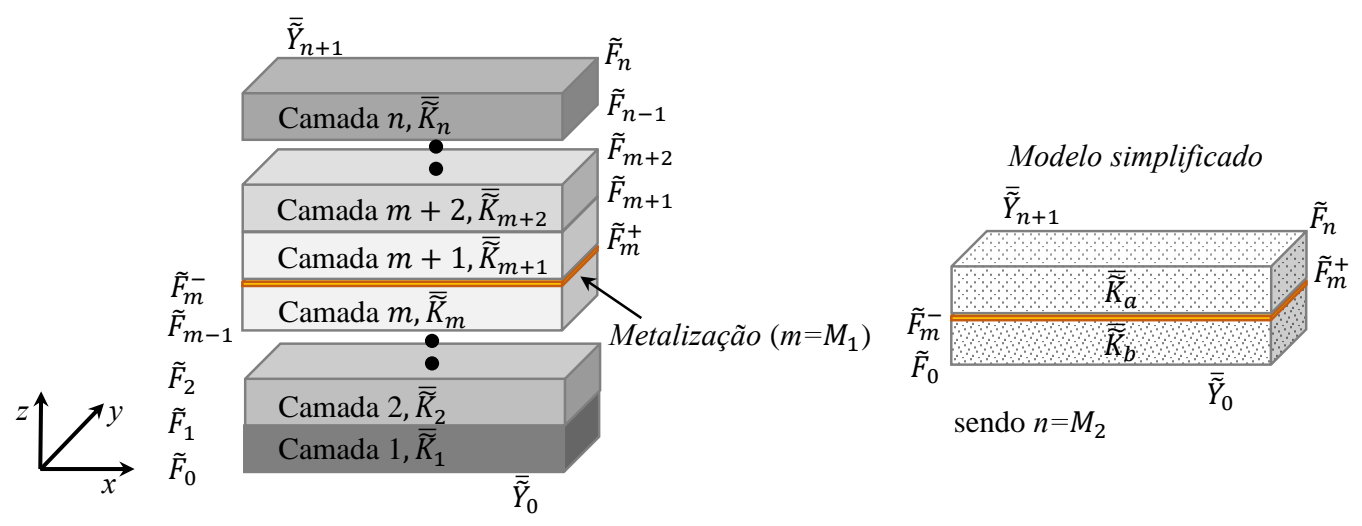

Figura 3.3: Meios estratificados separados por uma metalização e modelo simplificado.
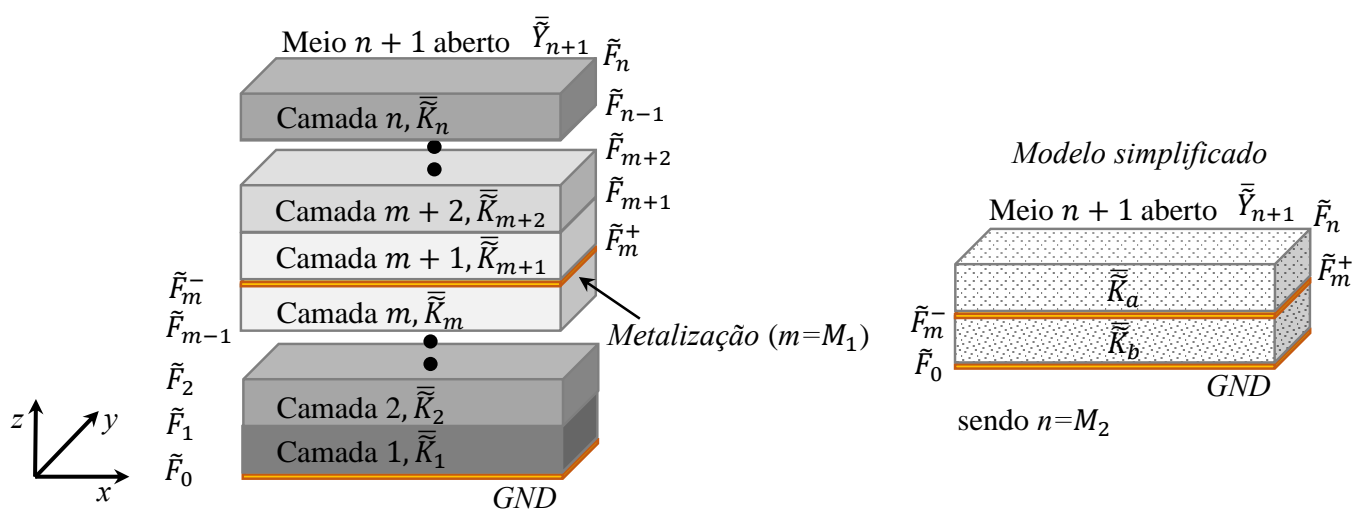

Figura 3.4: Estrutura de uma rede refletora multicamada e modelo simplicado.

sendo

$$
\overline{\tilde{Y}}^{(b)}=\overline{\tilde{B}}_{b} \overline{\tilde{Z}}_{b}^{-1}
$$

Caso a interface inferior estivesse aberta, então, a partir da (3-107), temse que $\tilde{\mathbf{H}}_{0}=-\overline{\tilde{Y}}_{0} \tilde{\mathbf{E}}_{0}$. Logo, a (3-124) torna-se

$$
\tilde{\mathbf{E}}_{m}^{-}=\left[\overline{\tilde{V}}_{b}+\overline{\tilde{Z}}_{b} \overline{\tilde{Y}}_{0}\right] \tilde{\mathbf{E}}_{0}
$$

$\mathrm{e}$

$$
\tilde{\mathbf{H}}_{m}^{-}=-\left[\overline{\tilde{Y}}_{b}+\overline{\tilde{B}}_{b} \overline{\tilde{Y}}_{0}\right] \tilde{\mathbf{E}}_{0} .
$$

Isolando $\tilde{\mathbf{E}}_{0}$ na (3-129) e substituindo na (3-130), faz com que o termo $\overline{\tilde{Y}}^{(b)}$ na (3-127) torne-se

$$
\overline{\tilde{Y}}^{(b)}=\left[\overline{\tilde{Y}}_{b}+\overline{\tilde{B}}_{b} \overline{\tilde{Y}}_{0}\right]\left[\overline{\tilde{V}}_{b}+\overline{\tilde{Z}}_{b} \overline{\tilde{Y}}_{0}\right]^{-1} .
$$

A análise para a parte superior da antena pode ser realizada de maneira análoga, supondo, neste caso, que a camada $n+1$ esteja aberta. Assim, utilizando a (3-123), obtém-se 


$$
\left[\begin{array}{c}
\tilde{\mathbf{E}}_{n} \\
\tilde{\mathbf{H}}_{n}
\end{array}\right]=\left[\begin{array}{cc}
\overline{\tilde{V}}_{a} & -\overline{\tilde{Z}}_{a} \\
-\tilde{\tilde{Y}}_{a} & \tilde{\tilde{B}}_{a}
\end{array}\right]\left[\begin{array}{c}
\tilde{\mathbf{E}}_{m}^{+} \\
\tilde{\mathbf{H}}_{m}^{+}
\end{array}\right]
$$

Uma relação entre os campos elétrico e magnético para um meio superior aberto foi estabelecida na (3-109), assim

e

$$
\tilde{\mathbf{E}}_{n}=\left[\begin{array}{ll}
\overline{\tilde{V}}_{a} & -\overline{\tilde{Z}}_{a}
\end{array}\right]\left[\begin{array}{c}
\tilde{\mathbf{E}}_{m}^{+} \\
\tilde{\mathbf{H}}_{m}^{+}
\end{array}\right]
$$

$$
\overline{\tilde{Y}}_{n+1} \tilde{\mathbf{E}}_{n}=\left[\begin{array}{ll}
-\overline{\tilde{Y}}_{a} & \overline{\tilde{B}}_{a}
\end{array}\right]\left[\begin{array}{c}
\tilde{\mathbf{E}}_{m}^{+} \\
\tilde{\mathbf{H}}_{m}^{+}
\end{array}\right] .
$$

Substituindo a (3-133) na (3-134), tem-se que

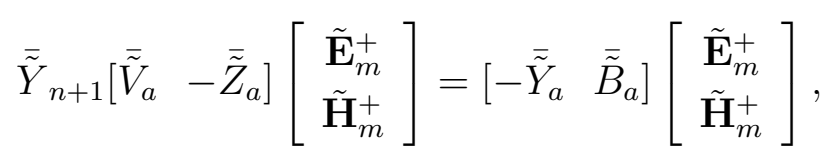

$\log 0$

$$
\tilde{\mathbf{H}}_{m}^{+}=\overline{\tilde{Y}}^{(a)} \tilde{\mathbf{E}}_{m}^{+}
$$

sendo

$$
\overline{\tilde{Y}}^{(a)}=\left[\overline{\tilde{Y}}_{n+1} \overline{\tilde{Z}}_{a}+\overline{\tilde{B}}_{a}\right]^{-1}\left[\overline{\tilde{Y}}_{n+1} \overline{\tilde{V}}_{a}+\overline{\tilde{Y}}_{a}\right] .
$$

Caso existisse na interface superior um plano de terra, então $\tilde{\mathbf{E}}_{n}=\mathbf{0}$. Assim $\overline{\tilde{Y}}^{(a)}$ da (3-136) torna-se,

$$
\overline{\tilde{Y}}^{(a)}=\overline{\tilde{Z}}_{a}^{-1} \overline{\tilde{V}}_{a} .
$$

Finalmente, as equações de continuidade apresentadas na (3-111) e (3112) devem ser levadas em consideração para se obter um sistema de equações desejado. Para o campo elétrico faz-se $\tilde{\mathbf{E}}_{m}^{-}=\tilde{\mathbf{E}}_{m}^{+}=\tilde{\mathbf{E}}_{m}$, assim

$$
\tilde{\mathbf{H}}_{m}^{-}=-\overline{\tilde{Y}}^{(b)} \tilde{\mathbf{E}}_{m}
$$

$\mathrm{e}$

$$
\tilde{\mathbf{H}}_{m}^{+}=\overline{\tilde{Y}}^{(a)} \tilde{\mathbf{E}}_{m}
$$

Substituindo (3-139) e (3-140) na (3-112), tem-se

$$
\tilde{\mathbf{E}}_{m}=\overline{\tilde{G}} \tilde{\mathbf{J}}^{(s)},
$$

em que

$$
\overline{\tilde{G}}=\left[\overline{\tilde{Y}}^{(a)}+\overline{\tilde{Y}}^{(b)}\right]^{-1}
$$

e corresponde à função diádica de Green no domínio espectral do problema. A equação (3-142) deverá ser utilizada no método da equação integral para se determinar a distribuição de corrente induzida na metalização, que, neste caso, é representada pelos diversos patches que formam uma rede refletora. 
Todas as possíveis combinações de solução podem ser representadas a partir de um modelo equivalente de duas portas, conforme esquemáticos ilustrados na Figura 3.5.
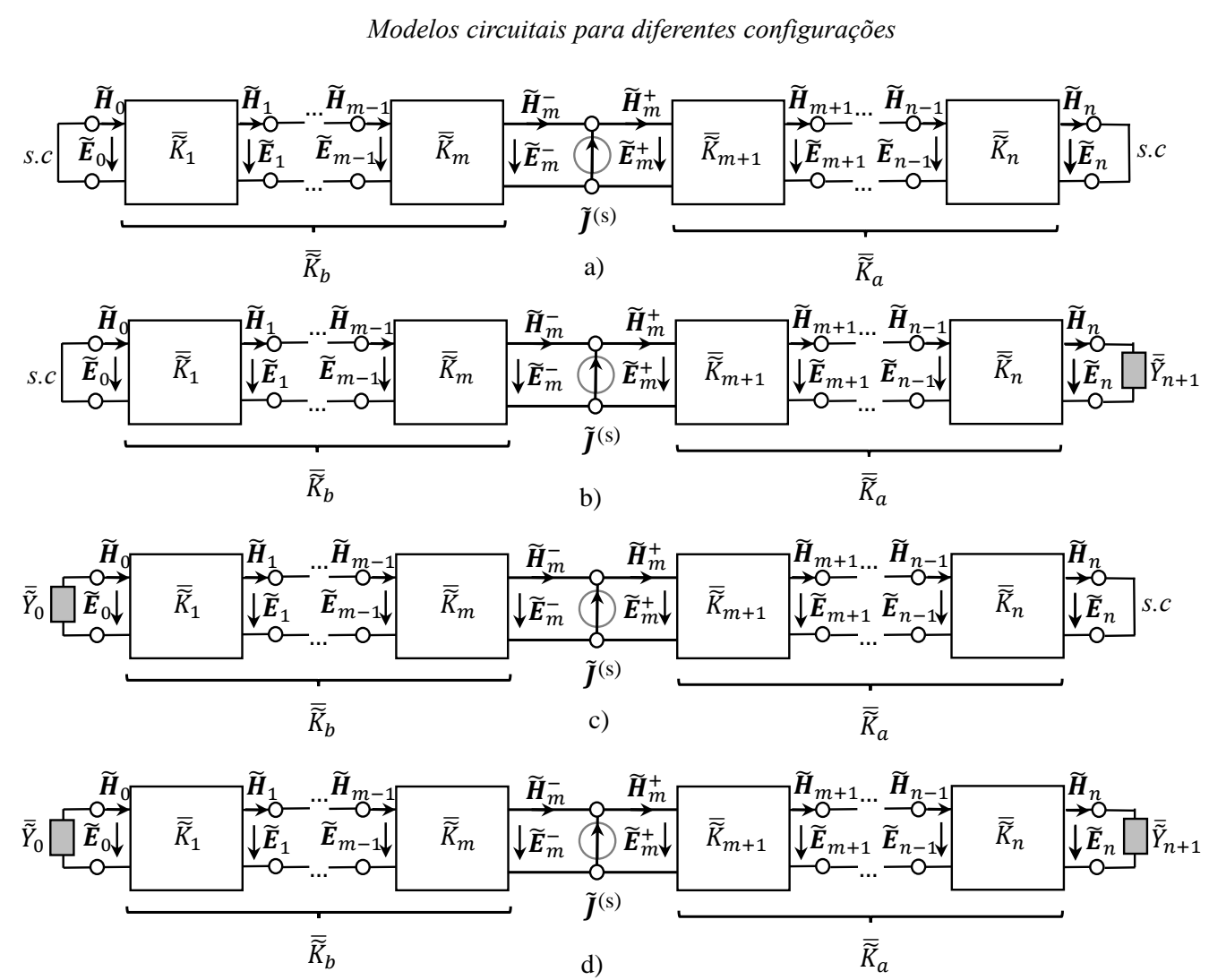

Condições de contorno na metalização

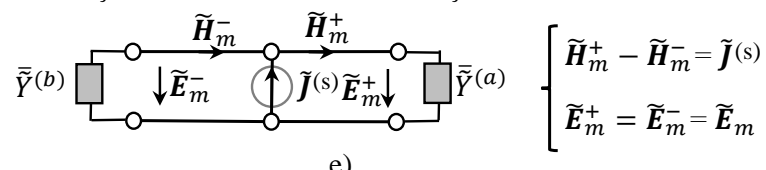

e)

Figura 3.5: Modelos circuitais de estrutura formada por camada(s) dielétrica(s), metalização e diferentes terminações (aberto ou com plano de terra). a) Stripline; b) Plano de terra na interface inferior e região superior aberta; c) Plano de terra na interface superior e região inferior aberta; d) Ambas as interfaces abertas; e) Admitâncias equivalentes vistas pela fonte.

\section{3}

\section{Validação numérica}

Sabendo-se da (3-141) que

$$
\overline{\tilde{Y}}^{(t)} \tilde{\mathbf{E}}_{m}=\tilde{\mathbf{J}}^{(s)},
$$

com $\overline{\tilde{Y}}^{(t)}=\left[\overline{\tilde{Y}}^{(a)}+\overline{\tilde{Y}}^{(b)}\right]$ e considerando uma estrutura sem metalizações (apenas o plano de terra), $\tilde{\mathbf{J}}^{(s)}=\mathbf{0}$. Soluções não-triviais são obtidas quando 
o determinante de $\overline{\tilde{Y}}^{(t)}$ for zero, denotado por $\operatorname{det}\left(\overline{\tilde{Y}}^{(t)}\right)=0$.

Para validar a função de Green obtida para uma estrutura formada por plano de terra, dielétrico e ar, resolve-se o problema apresentado na Figura 3.6 e considera-se $k_{\bar{x}}=0$. Os resultados obtidos são comparados com os ilustrados em [81], aplicando às seguintes relações: $\frac{d}{\lambda_{0}}=\frac{\bar{d}}{2 \pi} \mathrm{e} \frac{\beta}{k_{0}}=k_{\bar{y}}$.

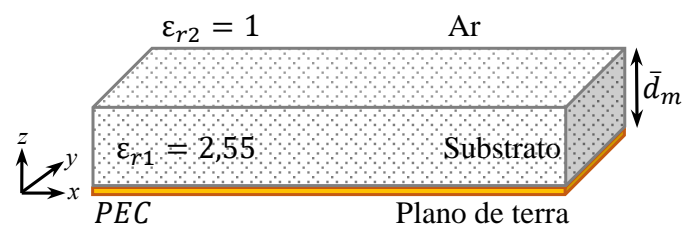

Figura 3.6: Geometria formada por plano de terra, substrato e ar.

O modelo circuital equivalente do problema é apresentado na Figura 3.7. As constantes de propagação dos seis primeiros modos que podem se estabelecer na estrutura com a variação da espessura do substrato foram determinados numericamente a partir do método de Müller. As curvas são apresentadas na Figura 3.8. Verifica-se apenas o modo fundamental (denominado $T M_{0}$ ) para a espessura normalizada $\left(\bar{d}_{m}\right)$ contida no intervalo de $\{0 ; 1,1\}$, uma vez que este modo se estabelece na estrutura independentemente da espessura do material. Vale salientar que o número de modos presentes depende simultaneamente da permissividade do material dielétrico, ou seja, quanto maior for a permissividade, maior poderá ser o número de modos para uma estrutura de mesma espessura. Adicionalmente, deve-se observar a frequência da fonte para definir quais os modos são evanescentes e propagantes.

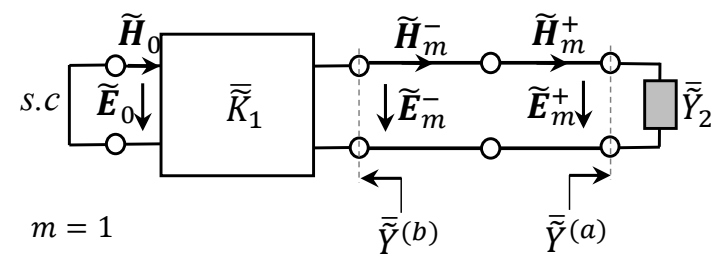

Figura 3.7: Modelo circuital para a estrutura da Figura 3.6.

Outra análise consiste em calcular as constantes de propagação em um guia retangular parcialmente preenchido constituído por paredes metálicas com condutividade infinita, conforme Figura 3.9. O modelo circuital é apresentado na Figura 3.10. Os modos de propagação são determinados para um guia retangular com as seguintes dimensões: $\bar{a}=5, \bar{b}=1,3 \bar{a}$, sendo $\bar{d}_{b}$ e $\bar{d}_{a}$ 


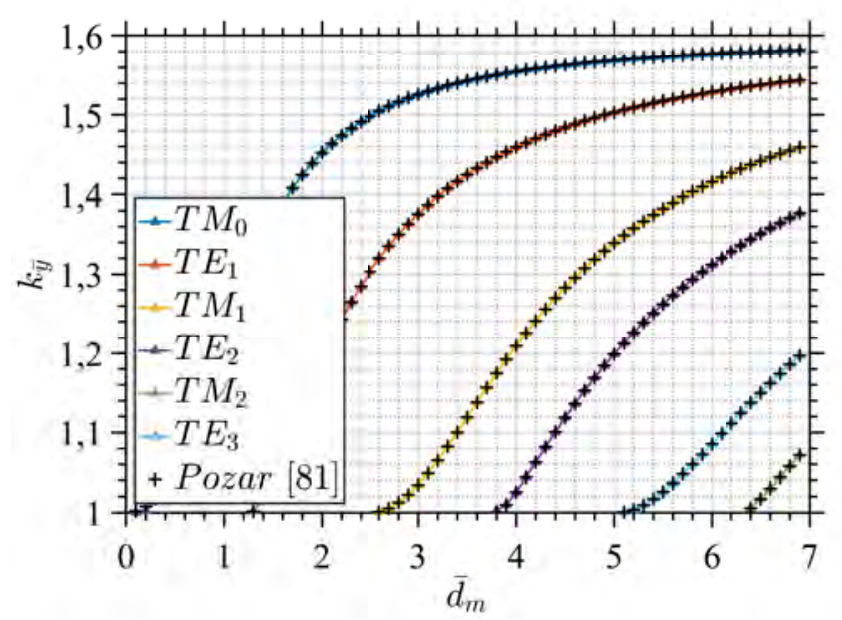

Figura 3.8: Constantes de propagação de ondas de superfície $\left(\varepsilon_{r}=2,55\right)$. Comparação com os resultados extraídos de [81].

variáveis a partir de um escalar $\alpha$ contido na faixa de 0 a 0,7 . Assume-se $q=0$ para se obter modos $T M_{p q}$ e $T E_{p q}$ dito puros (fazendo com que $k_{\bar{x}}$ seja zero). Do contrário, modos híbridos poderiam ser identificados. A partir disso, determinam-se os $p$ modos de propagação para $T M_{p 0}$ e $T E_{p 0}$ para $y$, respectivamente. Os resultados obtidos são apresentados na Figura 3.11, em que observa-se excelente concordância com os modos determinados a partir de equações características encontradas em [82].

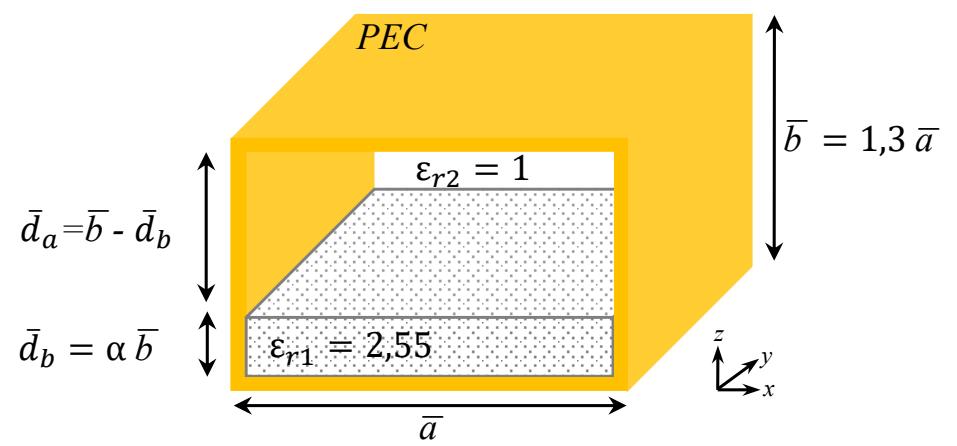

Figura 3.9: Guia retangular parcialmente preenchido. 


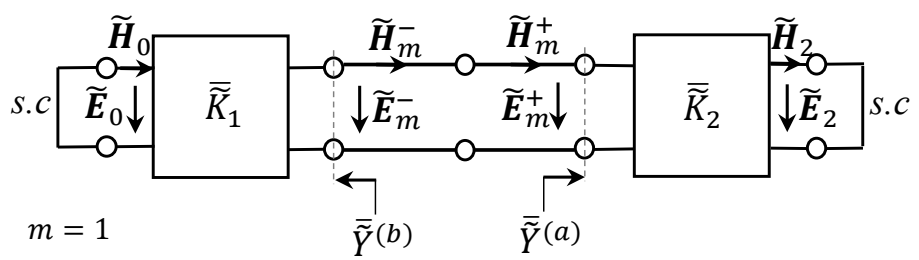

Figura 3.10: Modelo circuital para a estrutura da Figura 3.9.

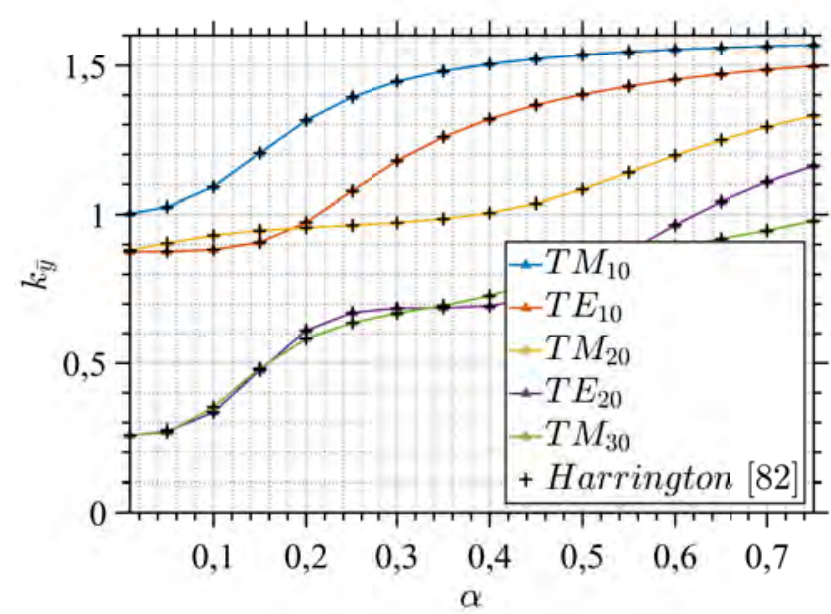

Figura 3.11: Constantes de propagação em guia retangular particialmente preenchido. Comparação com os resultados extraídos de [82].

No apêndice A encontra-se a formulação da função diádica de Green sem a consideração da normalização das dimensões espaciais e das constantes de propagação. 


\section{Análise de estruturas planares a partir do método dos mo- mentos - MoM}

O campo elétrico no domínio espectral descrito na (3-141) depende da função diádica de Green e da densidade de corrente elétrica superfícial. A função diádica de Green é determinada a partir das características físicas da estrutura multicamada, enquanto que a densidade de corrente elétrica é desconhecida na presença de uma metalização impressa sobre o dielétrico da Figura $3.6\left(\tilde{\mathbf{J}}^{(s)}\right.$ diferente de zero).

A distribuição de corrente pode ser determinada a partir da utilização de técnicas numéricas apropriadas. Aqui, o método dos momentos (MoM) é escolhido e formulado para se determinar o campo elétrico espalhado pela(s) densidade(s) de corrente elétrica que flui(em) sobre o(s) patch(es).

\section{1}

\section{Campo elétrico espalhado}

Neste trabalho, opta-se pelo projeto de uma rede refletora com o ajuste de fase a partir da variação das dimensões do elemento espalhador. A condição de contorno sobre a metalização impressa implica que o campo elétrico total tangencial deve ser zero. Matematicamente,

$$
\hat{z} \times\left.\mathbf{E}^{(T)}\right|_{z=z_{2}}=\mathbf{0}
$$

em que $z_{2}$ é a posição da interface na direção $z$ e $\mathbf{E}^{(T)}$ é o campo elétrico total descrito por

$$
\mathbf{E}^{(T)}=\mathbf{E}^{(i n c)}\left(\mathbf{J}^{(f)}\right)+\mathbf{E}^{(e s p)}\left(\mathbf{J}^{(s)}\right),
$$

sendo $\mathbf{E}^{(e s p)}$ o vetor campo elétrico espalhado por uma densidade de corrente superficial vetorial $\mathbf{J}^{(s)}$, que é originada pela descontinuidade das componentes do campo magnético tangencial no $m$-ésimo plano de metalização, e o $\mathbf{E}^{(i n c)}$ é o vetor campo elétrico incidente na metalização, que, neste caso, é gerado por uma densidade de corrente vetorial na fonte $\mathbf{J}^{(f)}$ (exemplo: antena corneta) e é constituído por duas parcelas

$$
\mathbf{E}^{(i n c)}\left(\mathbf{J}^{(f)}\right)=\mathbf{E}^{(f)}\left(\mathbf{J}^{(f)}\right)+\mathbf{E}^{(r)}\left(\mathbf{J}^{(f)}\right),
$$


Capítulo 4. Análise de estruturas planares a partir do método dos momentos MoM

sendo $\mathbf{E}^{(f)}$ o campo elétrico vetorial direto da fonte e $\mathbf{E}^{(r)}$ o campo elétrico vetorial transmitido através da interface ar-dielétrico e refletido no plano de terra. Desta forma, tem-se que

$$
\hat{y} E_{x}^{(T)}-\left.\hat{x} E_{y}^{(T)}\right|_{z=z_{2}}=\mathbf{0}
$$

Na Figura 4.1 pode ser visualizada a estrutura sendo iluminada por uma onda plana (TEM), enquanto que na Figura 4.2 é apresentado o modelo circuital para uma estrutura composta por uma camada dielétrica e uma metalização.

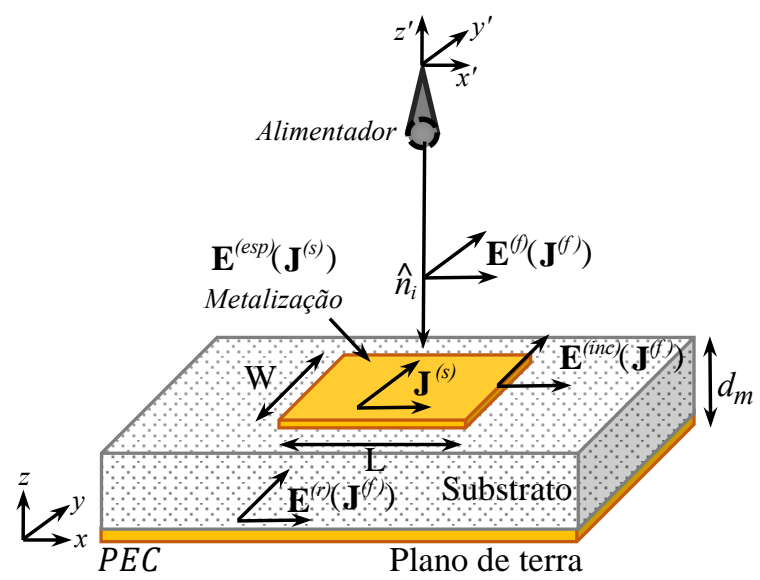

Figura 4.1: Campo elétrico tangencial total sobre a superfície metálica impressa.

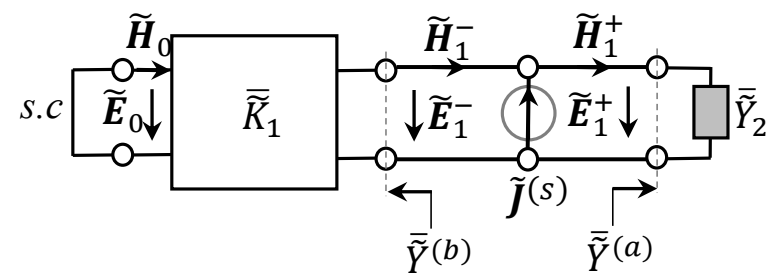

Figura 4.2: Modelo circuital para a estrutura da Figura 4.1.

Ambas as componentes do campo elétrico, $E_{x}^{(T)}$ e $E_{y}^{(T)}$, devem ser zero para satisfazer a igualdade. Desta forma, multiplicando a condição de contorno para o campo elétrico por uma função de teste (ou peso) conhecida, denominada $\mathbf{J}_{m}$, tem-se

$$
\left[\mathbf{E}^{(i n c)}\left(\mathbf{J}^{(f)}\right)+\mathbf{E}^{(e s p)}\left(\mathbf{J}^{(s)}\right)\right] .\left.\mathbf{J}_{m}\right|_{z=z_{2}}=0 .
$$

Fazendo o produto interno da (4-5), 


$$
\left\langle\left[\mathbf{E}^{(i n c)}\left(\mathbf{J}^{(f)}\right)+\mathbf{E}^{(e s p)}\left(\mathbf{J}^{(s)}\right)\right], \mathbf{J}_{m}\right\rangle=0
$$

obtém-se

$$
\iint_{S} \mathbf{E}^{(i n c)}\left(\mathbf{J}^{(f)}\right) . \mathbf{J}_{m} d S=-\iint_{S} \mathbf{E}^{(e s p)}\left(\mathbf{J}^{(s)}\right) . \mathbf{J}_{m} d S,
$$

sendo $S$ a superfície da metalização. A densidade de corrente superficial desconhecida sobre o patch pode ser descrita da seguinte forma

$$
\mathbf{J}^{(s)}=\hat{x} J_{x}+\hat{y} J_{y} .
$$

Reescrevendo em termos de funções base (ou expansão) conhecidas e de coeficientes a serem determinados, tem-se

$$
\mathbf{J}^{(s)}=\hat{x} \sum_{n_{x}=1}^{N_{x}} I_{n_{x}} J_{n_{x}}+\hat{y} \sum_{n_{y}=1}^{N_{y}} I_{n_{y}} J_{n_{y}}=\sum_{n=1}^{N_{y}+N_{x}} I_{n} \mathbf{J}_{n},
$$

sendo $N_{x}$ e $N_{y}$ os números de modos usados para a expansão da corrente ao longo de $x$ e $y$, e $\mathbf{J}_{n}$ é um vetor na direção de $y$ para todo $n$ pertencemente ao intervalo $\left\{1, N_{y}\right\}$ e na direção de $x$ para todo $n$ pertencemente ao intervalo $\left\{N_{y}+1, N_{y}+N_{x}\right\}$.

No domínio espectral,

$$
\tilde{\mathbf{J}}^{(s)}=\mathscr{F}\left(\mathbf{J}^{(s)}\right)=\hat{x} \mathscr{F}\left(J_{x}\right)+\hat{y} \mathscr{F}\left(J_{y}\right)=\hat{x} \tilde{J}_{x}+\hat{y} \tilde{J}_{y},
$$

em que $\mathscr{F}($.$) denota a transformada de Fourier da densidade de corrente$ superficial. Expandindo,

$$
\tilde{\mathbf{J}}^{(s)}=\hat{x} \sum_{n_{x}=1}^{N_{x}} I_{n_{x}} \tilde{J}_{n_{x}}+\hat{y} \sum_{n_{y}=1}^{N_{y}} I_{n_{y}} \tilde{J}_{n_{y}}=\sum_{n=1}^{N_{y}+N_{x}} I_{n} \tilde{\mathbf{J}}_{n} .
$$

Substituindo (4-9) em (4-7) e utilizando a propriedade da linearidade das equações de Maxwell, obtém-se a seguinte expressão

$$
\iint_{S} \mathbf{E}^{(i n c)}\left(\mathbf{J}^{(f)}\right) . \mathbf{J}_{m} d S=\sum_{n=1}^{N_{y}+N_{x}}-I_{n} \iint_{S} \mathbf{E}^{(e s p)}\left(\mathbf{J}_{n}\right) \cdot \mathbf{J}_{m} d S .
$$

$\mathrm{Na}$ forma matricial

$$
\bar{Z} \mathbf{I}=\mathbf{V}
$$

sendo $\mathbf{V}$ e $\mathbf{I}$ vetores coluna com dimensão $\left\{N_{y}+N_{x}\right\} \times 1$ e $\bar{Z}$ uma matriz com dimensão $\left\{N_{y}+N_{x}\right\} \times\left\{N_{y}+N_{x}\right\}$. A matriz $\bar{Z}$ contém as características da rede refletora em termos estruturais (espaçamentos, tamanho dos patches, etc.), V está relacionado ao tipo de excitação (iluminação) das metalizações e I está associado aos pesos das densidades de corrente superficiais e que devem ser determinados.

O vetor tensão para uma $m$-ésima função teste é descrito como sendo 
Capítulo 4. Análise de estruturas planares a partir do método dos momentos MoM

$$
\mathbf{V}_{m}=\iint_{S} \mathbf{E}^{(i n c)}\left(\mathbf{J}^{(f)}\right) . \mathbf{J}_{m} d S .
$$

Logo, pode-se representar para inúmeras funções teste como

$$
\mathbf{V}=\left[\begin{array}{llllll}
V_{1_{y}} \ldots V_{n_{y}} \ldots V_{N_{y_{y}}} & V_{1_{x}} \ldots V_{n_{x x}} \ldots V_{N_{x x}}
\end{array}\right]^{t}
$$

em que $[.]^{t}$ denota o vetor transposto, sendo cada posição do vetor calculada por

$$
V_{m_{i}}=\iint_{S} E_{i}^{(i n c)} J_{m_{i}} d S=\iint_{S}\left(E_{i}^{(f)}+E_{i}^{(r)}\right) J_{m_{i}} d S \quad i=y, x .
$$

O vetor corrente e a matriz impedância são descritos como sendo,

$$
\mathbf{I}=\left[\begin{array}{llllll}
I_{1_{y}} \ldots I_{n_{y_{y}}} \ldots I_{N_{y_{y}}} & I_{1_{x}} \ldots I_{n_{x x}} \ldots I_{N_{x x}}
\end{array}\right]_{N_{y}+N_{x}}^{t},
$$

em que $[.]^{t}$ denota o vetor transposto e

$$
\bar{Z}=\left[\begin{array}{cc}
\bar{Z}^{y y} & \bar{Z}^{y x} \\
\bar{Z}^{x y} & \bar{Z}^{x x}
\end{array}\right]
$$

sendo $\bar{Z}^{i j}$ submatrizes preenchidas a partir de

$$
Z_{m n}^{i j}=-\iint_{S} E_{i}^{(e s p)}\left(J_{n_{j}}\right) J_{m_{i}} d S \quad i, j=y, x .
$$

Usando (3-67), (3-112) e (3-141), tem-se que as componentes do campo elétrico no domínio espectral produzido pelas densidades de corrente elétrica são descritas por

$$
\left[\begin{array}{c}
\tilde{E}_{y} \\
\tilde{E}_{x}
\end{array}\right]=j \eta_{0} \overline{\tilde{G}}\left[\begin{array}{c}
\tilde{J}_{y} \\
\tilde{J}_{x}
\end{array}\right],
$$

em que a função diádica de Green $\overline{\tilde{G}}$ pode ser descrita da seguinte forma

$$
\overline{\tilde{G}}=\left[\begin{array}{cc}
\tilde{G}_{y y} & \tilde{G}_{y x} \\
\tilde{G}_{x y} & \tilde{G}_{x x}
\end{array}\right] .
$$

Assim,

$$
\left[\begin{array}{c}
\tilde{E}_{y} \\
\tilde{E}_{x}
\end{array}\right]=j \eta_{0}\left[\begin{array}{cc}
\tilde{G}_{y y} & \tilde{G}_{y x} \\
\tilde{G}_{x y} & \tilde{G}_{x x}
\end{array}\right]\left[\begin{array}{c}
\tilde{J}_{y} \\
\tilde{J}_{x}
\end{array}\right] .
$$

Sabendo-se que os campos eletromagnéticos são espalhados, reescreve-se

$$
\tilde{E}_{y}^{(e s p)}=j \eta_{0}\left[\tilde{G}_{y y} \tilde{J}_{y}+\tilde{G}_{y x} \tilde{J}_{x}\right]=\tilde{E}_{y}^{(e s p)}\left(\tilde{J}_{y}\right)+\tilde{E}_{y}^{(e s p)}\left(\tilde{J}_{x}\right)
$$

$\mathrm{e}$

$$
\tilde{E}_{x}^{(e s p)}=j \eta_{0}\left[\tilde{G}_{x y} \tilde{J}_{y}+\tilde{G}_{x x} \tilde{J}_{x}\right]=\tilde{E}_{x}^{(e s p)}\left(\tilde{J}_{y}\right)+\tilde{E}_{x}^{(e s p)}\left(\tilde{J}_{x}\right) .
$$

As densidades de corrente superficiais podem apresentar $n$ modos de expansão. Assim cada parcela do campo elétrico espalhado no domínio espectral 
Capítulo 4. Análise de estruturas planares a partir do método dos momentos MoM

para cada modo pode ser descrita na forma compacta por

$$
\tilde{E}_{i}^{(e s p)}\left(\tilde{J}_{n_{j}}\right)=j \eta_{0} \tilde{G}_{i j} \tilde{J}_{n_{j}} \quad i, j=y, x .
$$

Aplicando a transformada inversa dupla de Fourier é possível converter o campo elétrico do domínio espectral para o domínio espacial, como segue

$$
E_{i}^{(e s p)}\left(J_{n_{j}}\right)=\frac{1}{(2 \pi)^{2}} \int_{-\infty}^{+\infty} j \eta_{0} \tilde{G}_{i j} \tilde{J}_{n_{j}} e^{j k_{x} x} e^{j k_{y} y} d k_{x} d k_{y} \quad i, j=y, x .
$$

Substituindo (4-26) na (4-19), tem-se

$$
Z_{m n}^{i j}=\iint_{S}\left[\frac{-1}{(2 \pi)^{2}} \int_{-\infty}^{+\infty} j \eta_{0} \tilde{G}_{i j} \tilde{J}_{n_{j}} e^{j k_{x} x} e^{j k_{y} y} d k_{x} d k_{y}\right] J_{m_{i}} d S \quad i, j=y, x .
$$

A possibilidade de reescrever (4-27) pode ser observada, resultando em

$$
Z_{m n}^{i j}=\frac{-1}{(2 \pi)^{2}} \int_{-\infty}^{+\infty}\left[\iint_{S} J_{m_{i}} e^{j k_{x} x} e^{j k_{y} y} d S\right] j \eta_{0} \tilde{G}_{i j} \tilde{J}_{n_{j}} d k_{x} d k_{y} \quad i, j=x, y
$$

sendo o termo entre [.] a transformada de Fourier conjugada da função base, denotada por $\mathscr{F}\left(J_{m_{i}}^{*}\right)=\tilde{J}_{m_{i}}^{*}\left(k_{x}, k_{y}\right)$. Desta forma

$$
Z_{m n}^{i j}=\int_{-\infty}^{+\infty} \int_{i j} \mathscr{F}\left(J_{m_{i}}^{*}\right) \mathscr{F}\left(J_{n_{j}}\right) d k_{x} d k_{y} \quad i, j=x, y
$$

com

$$
Q_{i j}=-\frac{j \eta_{0} \tilde{G}_{i j}}{(2 \pi)^{2}}
$$

Neste trabalho a abordagem de Galerkin é aplicada, sendo as funções base iguais as de teste.

\subsection{1}

\section{Mapeamento e integração no plano complexo}

A integral numérica dupla de $-\infty$ a $+\infty$, apresentada na (4-29), é custosa computacionalmente. Uma alternativa é reescrever todas as expressões que envolvem $k_{x}$ e $k_{y}$ na forma polar, fazendo

$$
k_{x}=\beta \cos (\alpha)
$$

$\mathrm{e}$

$$
k_{y}=\beta \operatorname{sen}(\alpha)
$$

Assim a integral torna-se

$$
Z_{m n}^{i j}=\int_{0}^{2 \pi} \int_{0}^{+\infty} Q_{i j} \mathscr{F}\left(J_{m_{i}}^{*}\right) \mathscr{F}\left(J_{n_{j}}\right) \beta d \beta d \alpha .
$$

Ao longo do caminho de integração as condições de Sommerfeld devem ser 
satisfeitas. Desta forma, conforme abordado em [83], constata-se a existência de uma ramificação no plano complexo. Adicionalmente, pólos podem ser observados nas funções de Green, originados pelas ondas de superfície dos modos $T M$ e $T E$ que se estabelecem na estrutura. Conforme resultados ilustrados na Figura 3.8, as constantes de propagação destes modos estão contidas no intervalo de $k_{0}$ a $\sqrt{\varepsilon_{r_{b}}} k_{0}$, sendo $\varepsilon_{r_{b}}$ a permissidade equivalente dos substratos abaixo da metalização. Por fim, tem-se a integral que extende-se ao infinito.

Pelas características observadas anteriormente, é conveniente subdividir o caminho de integração numérica em três partes [84]. O primeiro intervalo é realizado na faixa $0 \leq \beta \leq k_{0}$. As constantes de propagação na direção de $z$ devem obedecer a condição de Sommerfeld, tal que

$$
k_{z_{n+1}}=-\sqrt{\beta^{2}-\varepsilon_{r_{n+1}} k_{0}^{2}}
$$

e

$$
k_{z_{b}}=\sqrt{-\left(\beta^{2}-\varepsilon_{r_{b}} k_{0}^{2}\right)},
$$

e as funções hiperbólicas das funções de Green devem ser substituídas por funções trigonométricas.

O segundo intervalo é realizado na faixa $k_{0} \leq \beta \leq \sqrt{\varepsilon_{r_{b}}} k_{0}$. As constantes são as mesmas do primeiro intervalo e as funções trigonométricas devem ser mantidas. O teorema dos resíduos deve ser aplicado em torno da(s) singularidade(s), as quais provocam mal condicionamento da função diádica de Green.

Da equação (3-119), tem-se que

$$
\overline{\tilde{K}}_{e q}=\left[\begin{array}{cc}
\overline{\tilde{V}}_{e q} & -\overline{\tilde{Z}}_{e q} \\
-\tilde{\tilde{Y}}_{e q} & \tilde{\tilde{B}}_{e q}
\end{array}\right],
$$

sendo

$\operatorname{com} \psi=V, B, Z$ ou $Y$.

$$
\overline{\tilde{\psi}}_{e q}=\left[\begin{array}{cc}
\tilde{\psi}_{e q}^{1,1} & \tilde{\psi}_{e q}^{1,2} \\
\tilde{\psi}_{e q}^{2,1} & \tilde{\psi}_{e q}^{2,2}
\end{array}\right]
$$

Da equação (3-142),

$$
\overline{\tilde{Y}}^{(a)}=\left[\begin{array}{cc}
\tilde{Y}_{1,1}^{(a)} & \tilde{Y}_{1,2}^{(a)} \\
\tilde{Y}_{2,1}^{(a)} & \tilde{Y}_{2,2}^{(a)}
\end{array}\right],
$$

desta forma, as funções de Green podem ser reescritas,

$$
\tilde{G}_{y y}=\frac{D\left[D \tilde{Y}_{2,2}^{(a)}+\tilde{B}_{e q}^{2,2} \tilde{Z}_{e q}^{1,1}\right]}{T_{\zeta}}=\frac{g_{y y}}{T_{\zeta}}
$$


$\mathrm{e}$

$$
\begin{aligned}
& \tilde{G}_{y x}=\frac{D\left[\tilde{B}_{e q}^{1,1} \tilde{Z}_{e q}^{1,2}-D \tilde{Y}_{1,2}^{(a)}\right]}{T_{\zeta}}=\frac{g_{y x}}{T_{\zeta}} \\
& \tilde{G}_{x y}=\frac{D\left[\tilde{B}_{e q}^{2,2} \tilde{Z}_{e q}^{2,1}-D \tilde{Y}_{2,1}^{(a)}\right]}{T_{\zeta}}=\frac{g_{x y}}{T_{\zeta}}
\end{aligned}
$$

sendo

$$
\tilde{G}_{x x}=\frac{D\left[D \tilde{Y}_{1,1}^{(a)}+\tilde{B}_{e q}^{1,1} \tilde{Z}_{e q}^{2,2}\right]}{T_{\zeta}}=\frac{g_{x x}}{T_{\zeta}},
$$

$$
D=\operatorname{det}\left(\overline{\tilde{Z}}_{e q}\right)=\tilde{Z}_{e q}^{1,1} \tilde{Z}_{e q}^{2,2}-\tilde{Z}_{e q}^{1,2} \tilde{Z}_{e q}^{2,1}
$$

e

$$
\begin{aligned}
T_{\zeta}=\left(D \tilde{Y}_{2,2}^{(a)}+\tilde{B}_{e q}^{2,2} \tilde{Z}_{e q}^{1,1}\right)\left(D \tilde{Y}_{1,1}^{(a)}+\tilde{B}_{e q}^{1,1} \tilde{Z}_{e q}^{2,2}\right) & \\
& -\left(D \tilde{Y}_{1,2}^{(a)}-\tilde{B}_{e q}^{1,1} \tilde{Z}_{e q}^{1,2}\right)\left(D \tilde{Y}_{2,1}^{(a)}-\tilde{B}_{e q}^{2,2} \tilde{Z}_{e q}^{2,1}\right) .
\end{aligned}
$$

O termo $T_{\zeta}$ é zero quando $\beta$ possuir o mesmo valor da constante de propagação que se estabelece na estrutura. Independentemente das características do substrato, é necessário analisar o termo do denominador, pois o modo $T M_{0}$ sempre estará presente. Em alguns casos, dependendo da espessura do substrato, diversos modos podem ser excitados.

Como $g_{i j}(i, j=x, y)$ e $T_{\zeta}$ são funções de $\alpha$ e $\beta$, reescreve-se

$$
\tilde{G}_{i j}(\alpha, \beta)=\frac{g_{i j}(\alpha, \beta)}{T_{\zeta}(\alpha, \beta)} \quad i, j=x, y
$$

e da (4-30) tem-se

$$
Q_{i j}(\alpha, \beta)=-\frac{j \eta_{0}}{(2 \pi)^{2}} \tilde{G}_{i j}(\alpha, \beta)
$$

Assim o integrando com singularidade(s) é descrito por

$$
U_{i j}(\alpha, \beta)=Q_{i j}(\alpha, \beta) \mathscr{F}\left(J_{m_{i}}^{*}(\alpha, \beta)\right) \mathscr{F}\left(J_{n_{j}}(\alpha, \beta)\right) \beta=\frac{u_{i j}(\alpha, \beta)}{T_{\zeta}(\alpha, \beta)} .
$$

Sabendo-se que a singularidade está relacionada com o modo de propagação, avalia-se a função em termos de $\beta$. Expandindo $T_{\zeta}$ em série de Taylor

$$
T_{\zeta}(\alpha, \beta)=T_{\zeta}\left(\alpha, \beta_{0}\right)+\left(\beta-\beta_{0}\right) T_{\zeta}^{\prime}\left(\alpha, \beta_{0}\right)+\frac{\left(\beta-\beta_{0}\right)^{2}}{2 !} T_{\zeta}^{\prime \prime}\left(\alpha, \beta_{0}\right)+\ldots
$$

Na singularidade $\beta_{0}$, referente ao modo $T M_{0}$, que sempre se propaga (ver Figura 3.8), o termo $T_{\zeta}\left(\alpha, \beta_{0}\right)=0$, logo truncando a expansão na (4-48) após o segundo termo, tem-se

$$
T_{\zeta}(\alpha, \beta) \approx\left(\beta-\beta_{0}\right) T_{\zeta}^{\prime}\left(\alpha, \beta_{0}\right)
$$

O teorema dos resíduos pode ser aplicado para computar o valor da integral em torno da singularidade em $\beta$, assim 


$$
\begin{aligned}
\operatorname{res}\left[\frac{u_{i j}(\alpha, \beta)}{T_{\zeta}(\alpha, \beta)}, \beta_{0}\right] & =\lim _{\beta \rightarrow \beta_{0}}\left(\beta-\beta_{0}\right) \frac{u_{i j}(\alpha, \beta)}{T_{\zeta}(\alpha, \beta)} \\
& =\lim _{\beta \rightarrow \beta_{0}}\left(\beta-\beta_{0}\right) \frac{u_{i j}(\alpha, \beta)}{\left(\beta-\beta_{0}\right) T_{\zeta}^{\prime}\left(\alpha, \beta_{0}\right)}
\end{aligned}
$$

resultando em

$$
\operatorname{res}\left[\frac{u_{i j}(\alpha, \beta)}{T_{\zeta}(\alpha, \beta)}, \beta_{0}\right]=\frac{u_{i j}\left(\alpha, \beta_{0}\right)}{T_{\zeta}^{\prime}\left(\alpha, \beta_{0}\right)},
$$

assim

$$
\oint_{C} U_{i j}(\alpha, \beta) d \beta= \pm j 2 \pi r e s\left[\frac{u_{i j}(\alpha, \beta)}{T_{\zeta}(\alpha, \beta)}, \beta_{0}\right]= \pm j 2 \pi \frac{u_{i j}\left(\alpha, \beta_{0}\right)}{T_{\zeta}^{\prime}\left(\alpha, \beta_{0}\right)},
$$

em que $C$ denota um caminho fechado em torno da singularidade e o sinal a ser escolhido depende do sentido de contorno (+ para anti-horário e - para horário). Em caso de existir $\vartheta$ singularidades, então reescreve-se como sendo

$$
\sum_{\xi=0}^{\vartheta-1} \oint_{C_{\xi}} U_{i j}(\alpha, \beta) d \beta= \pm j 2 \pi \sum_{\xi=0}^{\vartheta-1} \frac{u_{i j}\left(\alpha, \beta_{\xi}\right)}{T_{\zeta}^{\prime}\left(\alpha, \beta_{\xi}\right)} .
$$

No presente caso, o(s) pólo(s) se encontra $(\mathrm{m})$ sobre o eixo real (para substratos sem perdas), e o caminho percorrido pela integral em $\beta$ é de meio círculo no sentido horário. Sendo assim, tem-se

$$
\sum_{\xi=0}^{\vartheta-1} \frac{1}{2} \oint_{C_{\xi}} U_{i j}(\alpha, \beta) d \beta=-j \pi \sum_{\xi=0}^{\vartheta-1} \frac{u_{i j}\left(\alpha, \beta_{\xi}\right)}{T_{\zeta}^{\prime}\left(\alpha, \beta_{\xi}\right)} .
$$

A derivada $T_{\zeta}^{\prime}\left(\alpha, \beta_{\xi}\right)$ deve ser realizada numericamente. Como existe(m) singularidade(s), então é conveniente aplicar o conceito de derivada aproximada por forward difference ou backward difference, descritas como sendo

$$
T_{\zeta}^{\prime}\left(\alpha, \beta_{\xi}\right) \simeq \frac{T_{\zeta}\left(\alpha, \beta_{\xi}+\Delta \beta_{\xi}\right)-T_{\zeta}\left(\alpha, \beta_{\xi}\right)}{\Delta \beta_{\xi}}
$$

$\mathrm{Ou}$

$$
T_{\zeta}^{\prime}\left(\alpha, \beta_{\xi}\right) \simeq \frac{T_{\zeta}\left(\alpha, \beta_{\xi}\right)-T_{\zeta}\left(\alpha, \beta_{\xi}-\Delta \beta_{\xi}\right)}{\Delta \beta_{\xi}}
$$

respectivamente. $\mathrm{O}$ valor de variação $\Delta \beta_{\xi}$ deve ser escolhido adequadamente para que se tenha uma boa aproximação da derivada analítica.

O terceiro intervalo é realizado na faixa $\sqrt{\varepsilon_{r_{b}}} k_{0} \leq \beta \leq \mathscr{P} k_{0}$, sendo $\mathscr{P}$ um escalar contido na faixa $\left\{\sqrt{\varepsilon_{r_{b}}},+\infty\right\}$. As constantes são dadas por

$$
k_{z_{n+1}}=-\sqrt{\beta^{2}-\varepsilon_{r_{n+1}} k_{0}^{2}}
$$

$\mathrm{e}$

$$
k_{z_{b}}=\sqrt{\left(\beta^{2}-\varepsilon_{r_{b}} k_{0}^{2}\right)} .
$$

Entretanto, as funções hiperbólicas devem ser aplicadas.

A matriz impedância $Z_{m n}^{i j}$ é formada pelo somatório das integrais de cada intervalo, matematicamente 
Capítulo 4. Análise de estruturas planares a partir do método dos momentos MoM

$$
Z_{m n}^{i j}=\Im_{1}+\Im_{2}+\Im_{3}
$$

sendo

$$
\begin{gathered}
\Im_{1}=\int_{0}^{2 \pi} \int_{0}^{k_{0}} Q_{i j} \mathscr{F}\left(J_{m_{i}}^{*}\right) \mathscr{F}\left(J_{n_{j}}\right) \beta d \beta d \alpha, \\
\Im_{2}=\int_{0}^{2 \pi} \int_{k_{0}}^{\sqrt{\varepsilon_{r_{b}}} k_{0}} Q_{i j} \mathscr{F}\left(J_{m_{i}}^{*}\right) \mathscr{F}\left(J_{n_{j}}\right) \beta d \beta d \alpha
\end{gathered}
$$

$\mathrm{e}$

$$
\Im_{3}=\int_{0}^{2 \pi} \int_{\sqrt{\varepsilon_{r_{b}}} k_{0}}^{\mathscr{P} k_{0}} Q_{i j} \mathscr{F}\left(J_{m_{i}}^{*}\right) \mathscr{F}\left(J_{n_{j}}\right) \beta d \beta d \alpha .
$$

Sobre a integral $2\left(\Im_{2}\right)$, devido ao(s) pólo(s), é conveniente subdividir da seguinte forma,

$$
\Im_{2}=\Im_{2 I}+\Im_{2 M}+\Im_{2 F}+\Im_{2 R}
$$

sendo

$$
\begin{gathered}
\Im_{2 I}=\int_{0}^{2 \pi} \int_{k_{0}}^{\beta_{0}-\delta} Q_{i j} \mathscr{F}\left(J_{m_{i}}^{*}\right) \mathscr{F}\left(J_{n_{j}}\right) \beta d \beta d \alpha, \\
\Im_{2 F}=\int_{0}^{2 \pi} \int_{\beta_{(\vartheta-1)}+\delta}^{\sqrt{\varepsilon_{r_{b}}} k_{0}} Q_{i j} \mathscr{F}\left(J_{m_{i}}^{*}\right) \mathscr{F}\left(J_{n_{j}}\right) \beta d \beta d \alpha, \\
\Im_{2 R}=-j \pi \sum_{\xi=0}^{\vartheta-1} \int_{0}^{2 \pi} \frac{u_{i j}\left(\alpha, \beta_{\xi}\right)}{T_{\zeta}^{\prime}\left(\alpha, \beta_{\xi}\right)} d \alpha,
\end{gathered}
$$

$\mathrm{e}$

$$
\Im_{2 M}=\left\{\begin{array}{ll}
0 & \vartheta=1 \\
\sum_{\xi=1}^{\vartheta-1} \int_{0}^{2 \pi} \int_{\beta_{\xi-1}+\delta}^{\beta_{\xi}-\delta} Q_{i j} \mathscr{F}\left(J_{m_{i}}^{*}\right) \mathscr{F}\left(J_{n_{j}}\right) \beta d \beta d \alpha & \vartheta>1
\end{array},\right.
$$

em que $\delta$ corresponde a um fator de afastamento do caminho de integração em relação ao pólo. Um exemplo do plano complexo $\beta$ para uma estrutura planar que apresente três modos de propagação (três singularidades) pode ser visualizado na Figura 4.3.

Após determinada a matriz impedância e o vetor tensão, aplica-se (4-13) para se obter os pesos das correntes contidos no vetor I. Após calculados os coeficientes, a densidade de corrente superficial no domínio espacial é obtida fazendo uso da (4-8) e (4-9), enquanto que no domínio espectral da (4-10) e $(4-11)$. 
Capítulo 4. Análise de estruturas planares a partir do método dos momentos MoM

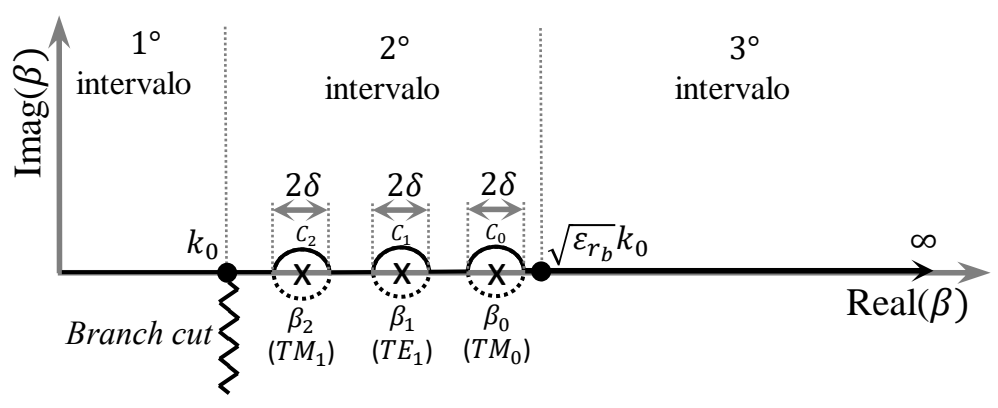

Figura 4.3: Exemplo de plano complexo para estrutura planar com três modos de propagação. Adaptado de [83].

\subsection{2}

\section{Rede refletora}

A abordagem do MoM para um elemento simples é aqui expandida para uma rede refletora planar composta por $E=E_{x} E_{y}$ elementos, sendo $E_{x}$ e $E_{y}$ o número de espalhadores em $x$ e $y$, respectivamente. Uma onda plana induzirá uma tensão ao incidir sobre o $p$-ésimo elemento, descrita matematicamente por

$$
\mathbf{V}_{m}^{p}=\iint_{S_{p}} \mathbf{E}^{p(i n c)}\left(\mathbf{J}^{(f)}\right) . \mathbf{J}_{m}^{p} d S_{p} .
$$

O vetor tensão é descrito da forma

$$
\mathbf{V}=\left[\mathbf{V}^{1} \ldots \mathbf{V}^{p} \ldots \mathbf{V}^{E}\right]^{t}
$$

com

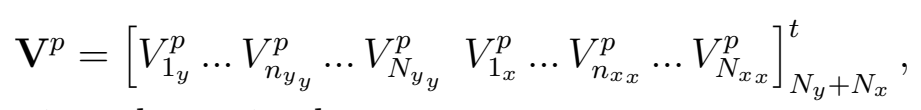

sendo cada posição determinada por

$$
V_{m_{i}}^{p}=\iint_{S_{p}} E_{i}^{p(i n c)} J_{m_{i}}^{p} d S_{p}=\iint_{S_{p}}\left(E_{i}^{p(f)}+E_{i}^{p(r)}\right) J_{m_{i}}^{p} d S_{p} \quad i=y, x .
$$

A equação (4-22) é reescrita na forma,

$$
\tilde{\mathbf{E}}^{q}=j \eta_{0} \overline{\tilde{G}} \tilde{\mathbf{J}}^{q}
$$

sendo

e

$$
\tilde{\mathbf{E}}^{q}=\left[\begin{array}{c}
\tilde{E}_{y}^{q} \\
\tilde{E}_{x}^{q}
\end{array}\right]
$$

$$
\tilde{\mathbf{J}}^{q}=\left[\begin{array}{c}
\tilde{J}_{y}^{q} \\
\tilde{J}_{x}^{q}
\end{array}\right]
$$

com $q=1,2, \ldots, E$. Assim, sabendo-se que os campos eletromagnéticos são espalhados, reescreve-se

$$
\tilde{E}_{y}^{(e s p)}=j \eta_{0} \sum_{q=1}^{E}\left[\tilde{G}_{y y} \tilde{J}_{y}^{q}+\tilde{G}_{y x} \tilde{J}_{x}^{q}\right]=\sum_{q=1}^{E}\left[\tilde{E}_{y}^{q(e s p)}\left(\tilde{J}_{y}^{q}\right)+\tilde{E}_{y}^{q(e s p)}\left(\tilde{J}_{x}^{q}\right)\right]
$$


Capítulo 4. Análise de estruturas planares a partir do método dos momentos -

$\mathrm{e}$

$$
\tilde{E}_{x}^{(e s p)}=j \eta_{0} \sum_{q=1}^{E}\left[\tilde{G}_{x y} \tilde{J}_{y}^{q}+\tilde{G}_{x x} \tilde{J}_{x}^{q}\right]=\sum_{q=1}^{E}\left[\tilde{E}_{x}^{q(e s p)}\left(\tilde{J}_{y}^{q}\right)+\tilde{E}_{x}^{q(e s p)}\left(\tilde{J}_{x}^{q}\right)\right] .
$$

Assumindo a possibilidade de existir $n$ modos para as densidades de corrente superficiais, cada parcela do campo elétrico espalhado no domínio espectral pode ser descrito na forma compacta por

$$
\tilde{E}_{i}^{q(e s p)}\left(\tilde{J}_{n_{j}}^{q}\right)=j \eta_{0} \tilde{G}_{i j} \tilde{J}_{n_{j}}^{q} \quad\left\{\begin{array}{l}
i, j=y, x \\
q=1,2, \ldots, E
\end{array} .\right.
$$

Escreve-se o campo elétrico no domínio espacial após aplicada a transformada inversa dupla de Fourier como sendo,

$$
E_{i}^{q(e s p)}\left(J_{n_{j}}^{q}\right)=\frac{1}{(2 \pi)^{2}} \int_{-\infty}^{+\infty} j \eta_{0} \tilde{G}_{i j} \tilde{J}_{n_{j}}^{q} e^{j k_{x} x} e^{j k_{y} y} d k_{x} d k_{y} .
$$

Substituindo (4-78) na (4-19), e considerando a existência de acoplamento eletromagnético entre os espalhadores da rede refletora, tem-se

$$
Z_{m n}^{i j, p q}=-\iint_{S_{p}}\left[\frac{1}{(2 \pi)^{2}} \int_{-\infty}^{+\infty} j \eta_{0} \tilde{G}_{i j} \tilde{J}_{n_{j}}^{q} e^{j k_{x} x} e^{j k_{y} y} d k_{x} d k_{y}\right] J_{m_{i}}^{p} d S_{p} .
$$

A possibilidade de reescrever (4-79) pode ser observada, resultando em

$$
Z_{m n}^{i j, p q}=-\frac{1}{(2 \pi)^{2}} \int_{-\infty}^{+\infty}\left[\iint_{S_{p}} J_{m_{i}}^{p} e^{j k_{x} x} e^{j k_{y} y} d S_{p}\right] j \eta_{0} \tilde{G}_{i j} \tilde{J}_{n_{j}}^{q} d k_{x} d k_{y},
$$

sendo o termo entre [.] a transformada de Fourier conjugada da função base, denotada por $\mathscr{F}\left(J_{m_{i}}^{p *}\right)=\tilde{J}_{m_{i}}^{p *}\left(k_{x}, k_{y}\right)$, desta forma

$$
Z_{m n}^{i j, p q}=\int_{-\infty}^{+\infty} \int_{i j} \mathscr{F}\left(J_{m_{i}}^{p *}\right) \mathscr{F}\left(J_{n_{j}}^{q}\right) d k_{x} d k_{y}
$$

com $Q_{i j}$ dado em (4-30).

\subsection{3}

\section{Funções base}

Para se obter eficiência na análise, funções base adequadas devem ser selecionadas de modo a reduzir a quantidade de funções necessárias para representar o comportamento da densidade de corrente superficial sobre a(s) metalização(ões) e, consequentemente, reduzir as dimensões da matriz impedância.

Em [85] são apresentados diversos tipos de funções base para a caracterização de redes refletoras infinitas formadas por patches ou dipolos cruzados 
impressos utilizando o MoM.

Conforme discutido em [86], funções base de domínio completo com condições de borda corretas podem ser utilizadas em análises de redes com elementos contendo formato canônico. Estas funções possibilitam a rápida convergência para um determinado número de funções base. Entretanto, devido ao comportamento singular nas bordas, o número de harmônicos de Floquet deve aumentar para representar o amplo espectro de Fourier. Já para elementos com forma arbitrária, as funções base de primeira ordem de Rao-Wilton-Glisson (RWG) ou rooftop podem ser utilizadas, porém a quantidade necessária é geralmente elevada.

Adicionalmente, em [86], um comparativo de performance na análise de uma RA $30 \times 30$ com patches retangulares utilizando MoM e fazendo uso das seguintes funções base foi apresentado: rooftops, Legendre de alta ordem de subdomínio, Legendre de alta ordem de domínio completo, funções senoidais e polinômios de primeiro e segundo tipo de Chebyshev. Como resultado, o melhor desempenho em termos de tempo computacional foi obtido utilizando funções base de domínio completo de Legendre com alta ordem, sendo 1,47 vezes mais rápido que polinômios de Chebyshev, 2,64 vezes mais rápido que funções senoidais de domínio completo, 5,58 vezes mais que funções base de subdomínio de Legendre com alta ordem, enquanto que funções de rooftops não apresentaram convergência.

Em [87] é apresentada uma função com condição de borda utilizada exclusivamente em antenas de microfita com formato retangular/quadrado. Já em [88] é realizada a segmentação da função proposta em [87]. Este tipo de representação possibilita a inclusão direta do comportamento impulsivo da densidade de corrente na borda do espalhador e necessita apenas da determinação de um fator de segmentação.

Neste trabalho, optou-se pela aplicação de espalhadores com formato quadrado. Redes refletoras possuem uma estrutura com grande similaridade às antenas de microfita, diferenciando-se principalmente na alimentação. Neste contexto, investiga-se funções base com condições de borda aplicadas na análise numérica de antenas de microfita com formato quadrado e com potencial em modelar a densidade de corrente em redes refletoras.

Em [89] é apresentado o emprego de funções base senoidais, as quais são descritas para o $q$-ésimo elemento como sendo

$\mathrm{e}$

$$
\begin{gathered}
J_{n x}^{q}(x, y)=J_{y}^{q}(y) \operatorname{sen}\left(\frac{n \pi}{L_{q}}\left(x-\left(x_{q}^{(o)}-L_{q} / 2\right)\right)\right) \\
J_{n y}^{q}(x, y)=J_{x}^{q}(x) \operatorname{sen}\left(\frac{n \pi}{W_{q}}\left(y-\left(y_{q}^{(o)}-W_{q} / 2\right)\right)\right),
\end{gathered}
$$


Capítulo 4. Análise de estruturas planares a partir do método dos momentos MoM

com

$$
\begin{aligned}
J_{y}^{q}(y) & =\frac{1}{W_{q}} \\
J_{x}^{q}(x) & =\frac{1}{L_{q}},
\end{aligned}
$$

sendo $L_{q}$ e $W_{q}$ as dimensões do $q$-ésimo espalhador metálico, $x_{q}^{(o)}$ e $y_{q}^{(o)}$ as coordenadas do centro do respectivo patch no plano $x y$, $x_{q}^{(o)}-L_{q} / 2<x<x_{q}^{(o)}+L_{q} / 2$ e $y_{q}^{(o)}-W_{q} / 2<y<y_{q}^{(o)}+W_{q} / 2$, enquanto que $n$ é a ordem do modo de propagação. Na Figura 4.4 são apresentados os quatro primeiros modos para a expansão da densidade de corrente superficial sobre a metalização.

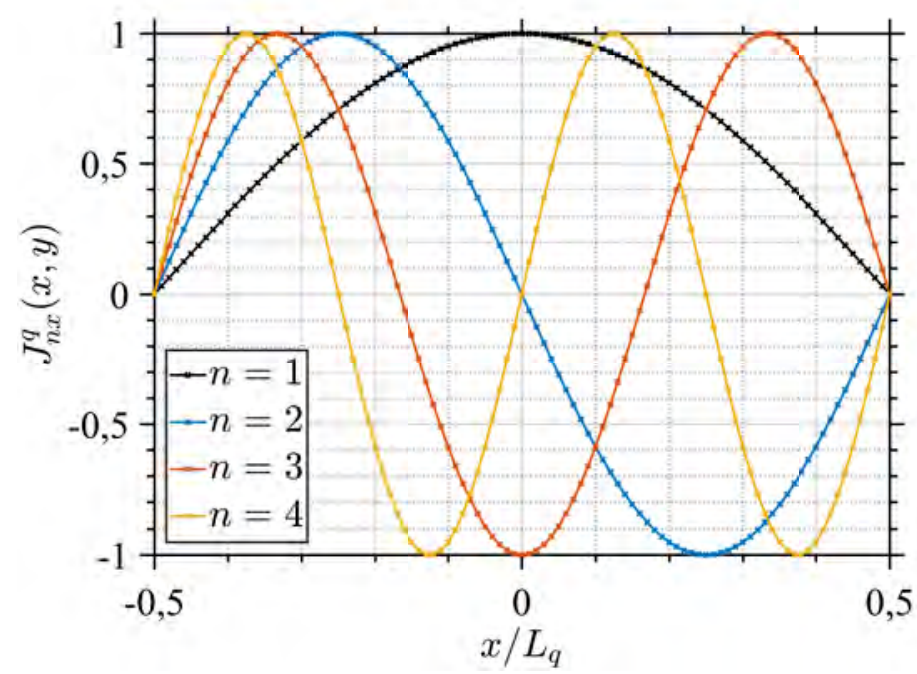

Figura 4.4: Quatro primeiros modos de expansão em $x$ no domínio completo para um espalhador centralizado com dimensão normalizada.

As distribuições de corrente ao longo de $x$ e $y$ para a análise com funções base com condições de borda, porém sem segmentação, conforme [87], requer que as funções $J_{y}^{q}(y)$ e $J_{x}^{q}(x)$ sejam descritas por,

$$
J_{y}^{q}(y)=\frac{1}{W_{q}}\left[1-\left(\frac{y-y_{q}^{(o)}}{W_{q} / 2}\right)^{2}\right]^{-1 / 2}
$$

$\mathrm{e}$

$$
J_{x}^{q}(x)=\frac{1}{L_{q}}\left[1-\left(\frac{x-x_{q}^{(o)}}{L_{q} / 2}\right)^{2}\right]^{-1 / 2},
$$

sendo o comportamento impulsivo da densidade de corrente na borda na direção transversal à $x$ ilustrado na Figura 4.5.

Outra possibilidade é aplicar uma função base com condição de borda segmentada, conforme [88], neste caso $J_{y}^{q}(y)$ e $J_{x}^{q}(x)$ tornam-se, 
Capítulo 4. Análise de estruturas planares a partir do método dos momentos MoM

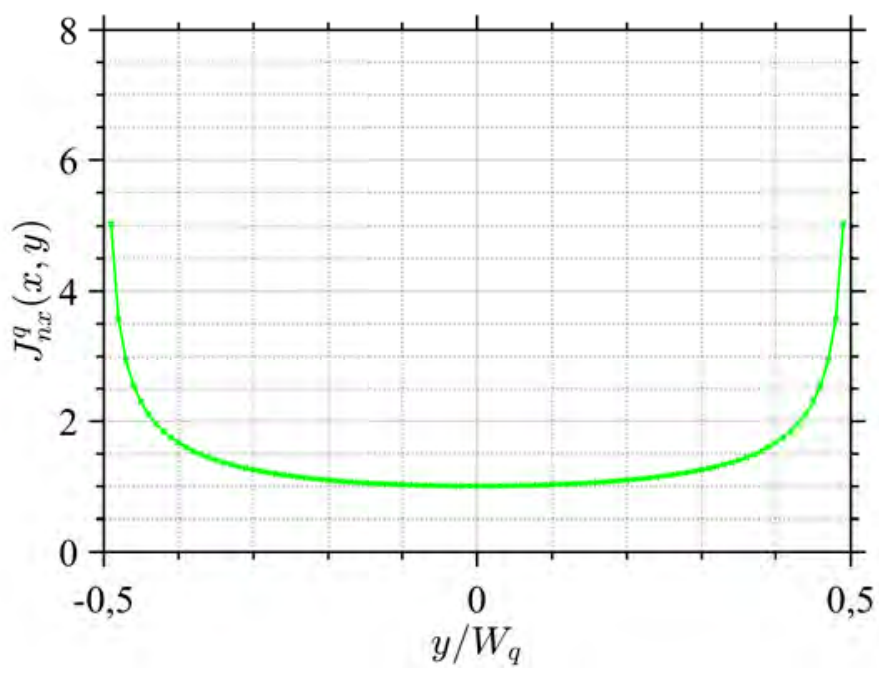

Figura 4.5: Condição de borda sem segmentação para uma distribuição de corrente no sentido de $x$.

$$
J_{\xi}^{q}(\xi)= \begin{cases}\frac{1}{U_{\xi}}\left[1-\left(\frac{\xi-\xi_{01}}{\xi_{b}}\right)^{2}\right]^{-1 / 2} & \text { com } \xi_{1}<\xi \leq \xi_{01} \\ \frac{1}{U_{\xi}} & \text { com } \xi_{01}<\xi<\xi_{02}, \\ \frac{1}{U_{\xi}}\left[1-\left(\frac{\xi-\xi_{02}}{\xi_{b}}\right)^{2}\right]^{-1 / 2} & \operatorname{com} \xi_{02} \leq \xi<\xi_{2}\end{cases}
$$

$\operatorname{com} \xi=x, y, U_{\xi}=L_{q}$ ou $W_{q}$,

$$
\begin{gathered}
\xi_{b}=\kappa\left|\frac{\xi_{2}-\xi_{1}}{2}\right| \\
\xi_{01}=\xi_{1}+\xi_{b} \\
\xi_{02}=\xi_{2}-\xi_{b}
\end{gathered}
$$

sendo que $\xi_{1}$ e $\xi_{2}$ denotam a posição inferior e superior da metalização na coordenada $\xi$.

O parâmetro $\kappa$ representa a região onde é aplicada a condição de borda, sendo a faixa de valores possíveis entre $0 \leq \kappa \leq 1$. Os resultados da variação deste parâmetro podem ser observados na Figura 4.6. Não há condição de borda se $\kappa=0$, equivalendo ao problema sem condição de borda (função senoidal). Caso $\kappa=1$, então a condição é aplicada em toda a largura do patch, resultando na formulação do problema com condição de borda sem segmentação.

A transformada dupla de Fourier para as funções base são descritas por,

$$
\tilde{J}_{n_{x}}^{q}\left(k_{x}, k_{y}\right)=\operatorname{sinc}\left(\frac{k_{y} W_{q}}{2}\right) \frac{n \pi}{L_{q}}\left[\frac{\cos (n \pi) e^{-j \frac{1}{2} k_{x} L_{q}}-e^{j \frac{1}{2} k_{x} L_{q}}}{k_{x}^{2}-\left(\frac{n \pi}{L_{q}}\right)^{2}}\right] \zeta_{q}^{\prime}
$$


Capítulo 4. Análise de estruturas planares a partir do método dos momentos MoM

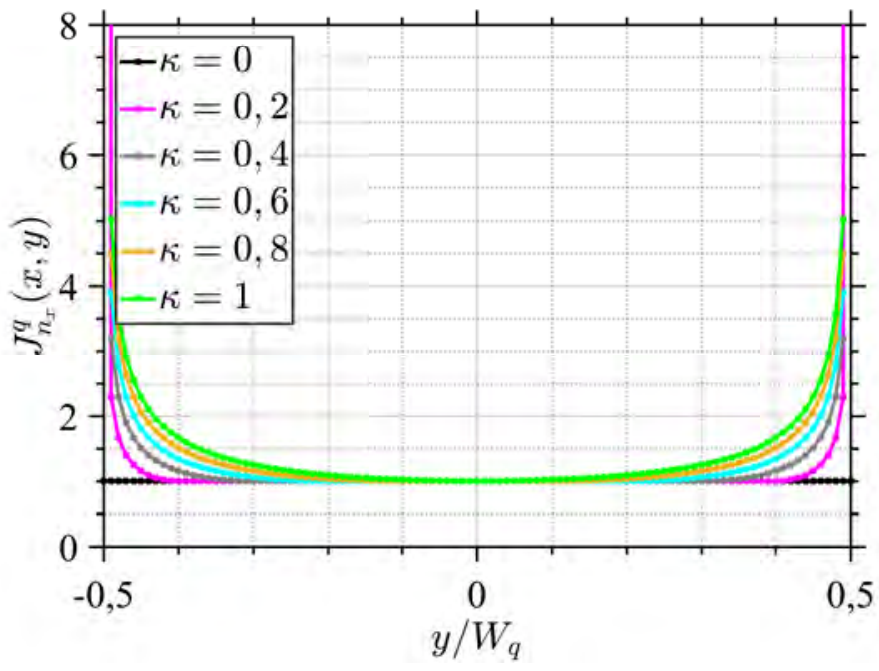

Figura 4.6: Condição de borda com segmentação para uma distribuição de corrente no sentido de $x$ aplicando diferentes valores de $\kappa$.

$\mathrm{e}$

$$
\tilde{J}_{n_{y}}^{q}\left(k_{x}, k_{y}\right)=\operatorname{sinc}\left(\frac{k_{x} L_{q}}{2}\right) \frac{n \pi}{W_{q}}\left[\frac{\cos (n \pi) e^{-j \frac{1}{2} k_{y} W_{q}}-e^{j \frac{1}{2} k_{y} W_{q}}}{k_{y}^{2}-\left(\frac{n \pi}{W_{q}}\right)^{2}}\right] \zeta_{q}^{\prime}
$$

para as senoidais,

$$
\tilde{J}_{n_{x}}^{q}\left(k_{x}, k_{y}\right)=\frac{\pi}{2} J_{0}\left(\frac{W_{q} k_{y}}{2}\right) \frac{n \pi}{L_{q}}\left[\frac{\cos (n \pi) e^{-j \frac{1}{2} k_{x} L_{q}}-e^{j \frac{1}{2} k_{x} L_{q}}}{k_{x}^{2}-\left(\frac{n \pi}{L_{q}}\right)^{2}}\right] \zeta_{q}^{\prime}
$$

e

$$
\tilde{J}_{n_{y}}^{q}\left(k_{x}, k_{y}\right)=\frac{\pi}{2} J_{0}\left(\frac{L_{q} k_{x}}{2}\right) \frac{n \pi}{W_{q}}\left[\frac{\cos (n \pi) e^{-j \frac{1}{2} k_{y} W_{q}}-e^{j \frac{1}{2} k_{y} W_{q}}}{k_{y}^{2}-\left(\frac{n \pi}{W_{q}}\right)^{2}}\right] \zeta_{q}^{\prime}
$$

para funções com condição de borda sem segmentação, e

$$
\tilde{J}_{n_{x}}^{q}\left(k_{x}, k_{y}\right)=\xi_{y}^{q}\left(k_{y}, W_{q}, \kappa\right) \frac{n \pi}{L_{q}}\left[\frac{\cos (n \pi) e^{-j \frac{1}{2} k_{x} L_{q}}-e^{j \frac{1}{2} k_{x} L_{q}}}{k_{x}^{2}-\left(\frac{n \pi}{L_{q}}\right)^{2}}\right] \zeta_{q}^{\prime},
$$

sendo

$$
\begin{aligned}
\xi_{y}^{q}\left(k_{y}, W_{q}, \kappa\right)= & (1-\kappa) \operatorname{sinc}\left(\frac{W_{q} k_{y}}{2}(-1+\kappa)\right)+ \\
& \frac{\pi \kappa}{2}\left[J_{0}\left(\frac{\kappa W_{q} k_{y}}{2}\right) \cos \left(\frac{W_{q} k_{y}}{2}(-1+\kappa)\right)\right. \\
& \left.\quad-j \mathscr{L}_{0}\left(\frac{\kappa W_{q} k_{y}}{2}\right) \operatorname{sen}\left(\frac{W_{q} k_{y}}{2}(-1+\kappa)\right)\right]
\end{aligned}
$$


$\mathrm{e}$

$$
\tilde{J}_{n_{y}}^{q}\left(k_{x}, k_{y}\right)=\xi_{x}^{q}\left(k_{x}, L_{q}, \kappa\right) \frac{n \pi}{W_{q}}\left[\frac{\cos (n \pi) e^{-j \frac{1}{2} k_{y} W_{q}}-e^{j \frac{1}{2} k_{y} W_{q}}}{k_{y}^{2}-\left(\frac{n \pi}{W_{q}}\right)^{2}}\right] \zeta_{q}^{\prime},
$$

sendo

$$
\begin{aligned}
& \xi_{x}^{q}\left(k_{x}, L_{q}, \kappa\right)=(1-\kappa) \operatorname{sinc}\left(\frac{L_{q} k_{x}}{2}(-1+\kappa)\right)+ \\
& \frac{\pi \kappa}{2}\left[J_{0}\left(\frac{\kappa L_{q} k_{x}}{2}\right) \cos \left(\frac{L_{q} k_{x}}{2}(-1+\kappa)\right)\right. \\
&\left.\quad-j \mathscr{L}_{0}\left(\frac{\kappa L_{q} k_{x}}{2}\right) \operatorname{sen}\left(\frac{L_{q} k_{x}}{2}(-1+\kappa)\right)\right]
\end{aligned}
$$

para as funções base com condição de borda segmentada, sendo $\zeta_{q}^{\prime}$ o deslocamento de fase devido a posição espacial do q-ésimo elemento, dado por

$$
\zeta_{q}^{\prime}=e^{-j\left(k_{x} x_{q}^{(o)}+k_{y} y_{q}^{(o)}\right)},
$$

$J_{0}$ representa a função de Bessel de primeira espécie e ordem zero e $\mathscr{L}_{0}$ denota a função Struve modificada de ordem zero, que é descrita matematicamente por

$$
\mathscr{L}_{0}(\gamma)=\frac{j 2}{\pi} \int_{0}^{\frac{\pi}{2}} \operatorname{sen}[\gamma \cos (v)] d v=j \mathscr{H}_{0}(\gamma),
$$

sendo $\mathscr{H}_{0}$ a função Struve de ordem zero. Vale destacar que a solução descrita implica que $\kappa$ seja maior que zero e que as expressões para as transformadas de Fourier diferem-se das apresentadas em [88]. A integral da (4-101) não possui solução analítica e deve ser resolvida numericamente. A transformada de Fourier conjugada das funções base, denotada por $\mathscr{F}\left(J_{m_{x}}^{p *}\right)=\tilde{J}_{m_{x}}^{p *}\left(k_{x}, k_{y}\right)$ ou $\mathscr{F}\left(J_{m_{y}}^{p *}\right)=\tilde{J}_{m_{y}}^{p *}\left(k_{x}, k_{y}\right)$, são descritas como sendo

$$
\tilde{J}_{m_{x}}^{q *}\left(k_{x}, k_{y}\right)=\operatorname{sinc}\left(\frac{k_{y} W_{q}}{2}\right) \frac{m \pi}{L_{q}}\left[\frac{\cos (m \pi) e^{j \frac{1}{2} k_{x} L_{q}}-e^{-j \frac{1}{2} k_{x} L_{q}}}{k_{x}^{2}-\left(\frac{m \pi}{L_{q}}\right)^{2}}\right] \zeta_{q}^{*}
$$

e

$$
\tilde{J}_{m_{y}}^{q *}\left(k_{x}, k_{y}\right)=\operatorname{sinc}\left(\frac{k_{x} L_{q}}{2}\right) \frac{m \pi}{W_{q}}\left[\frac{\cos (m \pi) e^{j \frac{1}{2} k_{y} W_{q}}-e^{-j \frac{1}{2} k_{y} W_{q}}}{k_{y}^{2}-\left(\frac{m \pi}{W_{q}}\right)^{2}}\right] \zeta_{q}^{*}
$$

para funções base senoidais,

$$
\tilde{J}_{m_{x}}^{p *}\left(k_{x}, k_{y}\right)=\frac{\pi}{2} J_{0}\left(\frac{W_{p} k_{y}}{2}\right) \frac{m \pi}{L_{p}}\left[\frac{\cos (m \pi) e^{j \frac{1}{2} k_{x} L_{p}}-e^{-j \frac{1}{2} k_{x} L_{p}}}{k_{x}^{2}-\left(\frac{m \pi}{L_{p}}\right)^{2}}\right] \zeta_{q}^{*}
$$


Capítulo 4. Análise de estruturas planares a partir do método dos momentos MoM

$$
\tilde{J}_{m_{y}}^{p *}\left(k_{x}, k_{y}\right)=\frac{\pi}{2} J_{0}\left(\frac{L_{p} k_{x}}{2}\right) \frac{m \pi}{W_{p}}\left[\frac{\cos (m \pi) e^{j \frac{1}{2} k_{y} W_{p}}-e^{-j \frac{1}{2} k_{y} W_{p}}}{k_{y}^{2}-\left(\frac{m \pi}{W_{p}}\right)^{2}}\right] \zeta_{q}^{*}
$$

para funções base com condições de borda sem segmentação e

$$
\tilde{J}_{m_{x}}^{p *}\left(k_{x}, k_{y}\right)=\xi_{y}^{p}\left(k_{y}, W_{p}, \kappa\right) \frac{m \pi}{L_{q}}\left[\frac{\cos (m \pi) e^{j \frac{1}{2} k_{x} L_{p}}-e^{-j \frac{1}{2} k_{x} L_{p}}}{k_{x}^{2}-\left(\frac{m \pi}{L_{p}}\right)^{2}}\right] \zeta_{q}^{\prime *}
$$

$\mathrm{e}$

$$
\tilde{J}_{m_{y}}^{p *}\left(k_{x}, k_{y}\right)=\xi_{x}^{p}\left(k_{x}, L_{p}, \kappa\right) \frac{m \pi}{W_{p}}\left[\frac{\cos (m \pi) e^{j \frac{1}{2} k_{y} W_{p}}-e^{-j \frac{1}{2} k_{y} W_{p}}}{k_{y}^{2}-\left(\frac{m \pi}{W_{p}}\right)^{2}}\right] \zeta_{q}^{\prime *}
$$

para funções base com condições de borda segmentada, sendo $\zeta_{q}^{\prime *}$ o complexo conjugado da (4-100).

\section{2}

\section{Campos elétricos incidente e refletido}

Uma onda plana gerada a partir de uma densidade de corrente em uma fonte $\mathbf{J}^{(f)}$ incide sobre a estrutura apresentada na Figura 4.7. A partir disso, pode-se calcular os campos incidente, refletido e transmitido nas diferentes regiões através da combinação do formalismo apresentado em [90,91]. Para a respectiva análise, o campo elétrico para cada polarização é verificado separadamente, visto que as equações de Maxwell são lineares.

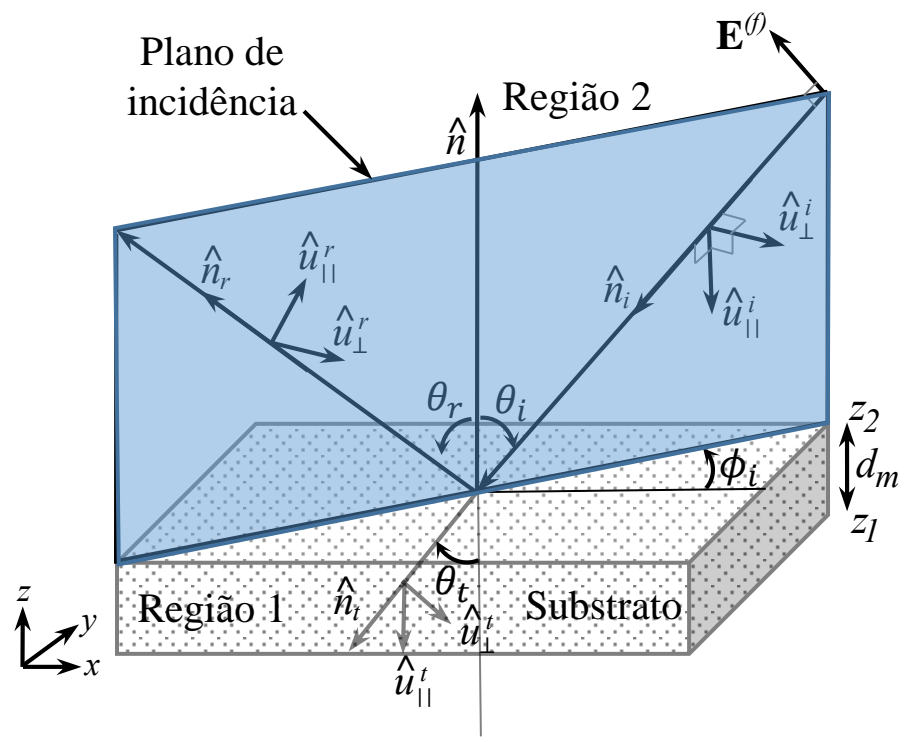

Figura 4.7: Reflexão em uma interface que separa dois meios distintos.

Os campos eletromagnéticos de uma onda plana incidente gerada pela 
Capítulo 4. Análise de estruturas planares a partir do método dos momentos MoM

fonte são descritos matematicamente da forma,

$$
\begin{gathered}
\mathbf{E}^{(f)}=\mathbf{E}_{0}^{i} e^{-j \mathbf{k}^{i} \cdot \mathbf{r}} \\
\mathbf{H}^{(f)}=\frac{1}{\eta_{2}}\left[\hat{n}_{i} \times \mathbf{E}^{(f)}\right],
\end{gathered}
$$

$\mathrm{e}$

sendo $\mathbf{E}_{0}^{i}$ o vetor amplitude do campo elétrico incidente, representado por

$$
\mathbf{E}_{0}^{i}=E_{0_{\|}} \hat{u}_{\|}^{i}+E_{0_{\perp}} \hat{u}_{\perp}^{i}
$$

com

$$
\hat{u}_{\|}^{i}=\hat{x} \cos \left(\theta_{i}\right) \cos \left(\phi_{i}\right)+\hat{y} \cos \left(\theta_{i}\right) \operatorname{sen}\left(\phi_{i}\right)-\hat{z} \operatorname{sen}\left(\theta_{i}\right)
$$

e

$$
\hat{u}_{\perp}^{i}=\hat{x} \operatorname{sen}\left(\phi_{i}\right)-\hat{y} \cos \left(\phi_{i}\right),
$$

em que $\perp$ e $\|$ indicam as direções perpendicular e paralela ao plano de incidência, respectivamente. Já r é o vetor posição, descrito por

$$
\mathbf{r}=\hat{x} x+\hat{y} y+\hat{z} z
$$

e $\mathbf{k}^{i}$ o vetor propagação

$$
\mathbf{k}^{i}=k_{2} \hat{n}_{i}=\left(-\hat{x} k_{x_{2}}^{i}-\hat{y} k_{y_{2}}^{i}-\hat{z} k_{z_{2}}^{i}\right)
$$

$\operatorname{com~} k_{x_{2}}^{i}=k_{2} \operatorname{sen}\left(\theta_{i}\right) \cos \left(\phi_{i}\right), k_{y_{2}}^{i}=k_{2} \operatorname{sen}\left(\theta_{i}\right) \operatorname{sen}\left(\phi_{i}\right)$ e $k_{z_{2}}^{i}=k_{2} \cos \left(\theta_{i}\right)$. Para este caso, em que a região 2 é o espaço livre, tem-se que $k_{2}=k_{0}$ e $\eta_{2}=\eta_{0}$.

Os campos eletromagnéticos refletidos são dados por

$$
\begin{gathered}
\mathbf{E}^{(r)}=\mathbf{E}_{0}^{r} e^{-j \mathbf{k}^{r} \cdot \mathbf{r}} \\
\mathbf{H}^{(r)}=\frac{1}{\eta_{2}}\left[\hat{n}_{r} \times \mathbf{E}^{(r)}\right],
\end{gathered}
$$

sendo $\mathbf{E}_{0}^{r}$ o vetor amplitude do campo elétrico refletido, dado por

$$
\mathbf{E}_{0}^{r}=\Gamma_{21}^{\|} E_{0 \|} \hat{u}_{\|}^{r}+\Gamma_{21}^{\perp} E_{0_{\perp}} \hat{u}_{\perp}^{r}
$$

com

$$
\hat{u}_{\|}^{r}=\hat{x} \cos \left(\theta_{r}\right) \cos \left(\phi_{r}\right)+\hat{y} \cos \left(\theta_{r}\right) \operatorname{sen}\left(\phi_{r}\right)+\hat{z} \operatorname{sen}\left(\theta_{r}\right)
$$

$\mathrm{e}$

$$
\hat{u}_{\perp}^{r}=\hat{x} \operatorname{sen}\left(\phi_{r}\right)-\hat{y} \cos \left(\phi_{r}\right) .
$$

O vetor posição é descrito na (4-113) e $\mathbf{k}^{r}$ é o vetor propagação

$$
\mathbf{k}^{r}=k_{2} \hat{n}_{r}=\left(-\hat{x} k_{x_{2}}^{r}-\hat{y} k_{y_{2}}^{r}+\hat{z} k_{z_{2}}^{r}\right),
$$

$\operatorname{com} k_{x_{2}}^{r}=k_{2} \operatorname{sen}\left(\theta_{r}\right) \cos \left(\phi_{r}\right), k_{y_{2}}^{r}=k_{2} \operatorname{sen}\left(\theta_{r}\right) \operatorname{sen}\left(\phi_{r}\right)$ e $k_{z_{2}}^{r}=k_{2} \cos \left(\theta_{r}\right)$.

Os campos eletromagnéticos de uma onda plana transmitidos para a região 1 são

$$
\mathbf{E}^{(t)}=\mathbf{E}_{0}^{t} e^{-j \mathbf{k}^{t} \cdot \mathbf{r}}
$$


Capítulo 4. Análise de estruturas planares a partir do método dos momentos MoM

$\mathrm{e}$

$$
\mathbf{H}^{(t)}=\frac{1}{\eta_{1}}\left[\hat{n}_{t} \times \mathbf{E}^{(t)}\right]
$$

sendo $\mathbf{E}_{0}^{t}$ o vetor amplitude do campo elétrico transmitido, descrito como

$$
\mathbf{E}_{0}^{t}=T_{12}^{\|} E_{0_{\|}} \hat{u}_{\|}^{t}+T_{12}^{\perp} E_{0_{\perp}} \hat{u}_{\perp}^{t},
$$

com

$$
\hat{u}_{\|}^{t}=\hat{x} \cos \left(\theta_{t}\right) \cos \left(\phi_{t}\right)+\hat{y} \cos \left(\theta_{t}\right) \operatorname{sen}\left(\phi_{t}\right)-\hat{z} \operatorname{sen}\left(\theta_{t}\right)
$$

$\mathrm{e}$

$$
\hat{u}_{\perp}^{t}=\hat{x} \operatorname{sen}\left(\phi_{t}\right)-\hat{y} \cos \left(\phi_{t}\right),
$$

sendo o vetor posição descrito na (4-113) e $\mathbf{k}^{t}$ é o vetor propagação

$$
\mathbf{k}^{t}=k_{1} \hat{n}_{t}=\left(-\hat{x} k_{x_{1}}^{t}-\hat{y} k_{y_{1}}^{t}-\hat{z} k_{z_{1}}^{t}\right)
$$

$\operatorname{com} k_{x_{1}}^{t}=k_{1} \operatorname{sen}\left(\theta_{t}\right) \cos \left(\phi_{t}\right), k_{y_{1}}^{t}=k_{1} \operatorname{sen}\left(\theta_{t}\right) \operatorname{sen}\left(\phi_{t}\right)$ e $k_{z_{1}}^{t}=k_{1} \cos \left(\theta_{t}\right)$.

Realizando o casamento de fase dos campos totais em cada região na interface que separa ambos os meios, tem-se que $\theta_{i}=\theta_{r}, \phi_{i}=\phi_{r}=\phi_{t} \mathrm{e}$ $k_{2} \operatorname{sen}\left(\theta_{i}\right)=k_{1} \operatorname{sen}\left(\theta_{t}\right)$, conhecidas como leis de Snell da reflexão e refração. Assim,

$$
\begin{aligned}
& \hat{u}_{\|}^{i}=\hat{x} \cos \left(\theta_{i}\right) \cos \left(\phi_{i}\right)+\hat{y} \cos \left(\theta_{i}\right) \operatorname{sen}\left(\phi_{i}\right)-\hat{z} \operatorname{sen}\left(\theta_{i}\right), \\
& \hat{u}_{\|}^{r}=\hat{x} \cos \left(\theta_{i}\right) \cos \left(\phi_{i}\right)+\hat{y} \cos \left(\theta_{i}\right) \operatorname{sen}\left(\phi_{i}\right)+\hat{z} \operatorname{sen}\left(\theta_{i}\right), \\
& \hat{u}_{\|}^{t}=\hat{x} \cos \left(\theta_{t}\right) \cos \left(\phi_{i}\right)+\hat{y} \cos \left(\theta_{t}\right) \operatorname{sen}\left(\phi_{i}\right)-\hat{z} \operatorname{sen}\left(\theta_{t}\right)
\end{aligned}
$$

$\mathrm{e}$

$$
\hat{u}_{\perp}^{i}=\hat{u}_{\perp}^{r}=\hat{u}_{\perp}^{t}=\hat{x} \operatorname{sen}\left(\phi_{i}\right)-\hat{y} \cos \left(\phi_{i}\right) .
$$

Adicionalmente, o casamento de fase dos campos eletromagnéticos na interface entre as regiões 2 e 1 implica que $k_{x_{1}}^{t}=k_{x_{2}}^{i}=k_{0_{x}}$ e $k_{y_{1}}^{t}=k_{y_{2}}^{i}=k_{0_{y}}$.

Aplicando as condições de contorno para os campos eletromagnéticos na interface que separa as duas regiões, tem-se que os coeficientes de transmissão e reflexão para ambas as polarizações são descritos como sendo

$\mathrm{e}$

$$
\begin{aligned}
\Gamma_{a b}^{\perp} & =\frac{\mu_{b} k_{z_{a}}^{i}-\mu_{a} k_{z_{b}}^{t}}{\mu_{b} k_{z_{a}}^{i}+\mu_{a} k_{z_{b}}^{t}}, \\
\Gamma_{a b}^{\|} & =\frac{\varepsilon_{a} k_{z_{b}}^{t}-\varepsilon_{b} k_{z_{a}}^{i}}{\varepsilon_{a} k_{z_{b}}^{t}+\varepsilon_{b} k_{z_{a}}^{i}}, \\
T_{a b}^{\perp} & =\frac{2 \mu_{a} k_{z_{b}}^{t}}{\mu_{b} k_{z_{a}}^{i}+\mu_{a} k_{z_{b}}^{t}} \\
T_{a b}^{\|} & =\frac{2 \sqrt{\varepsilon_{a} \varepsilon_{b}} k_{z_{b}}^{t}}{\varepsilon_{a} k_{z_{b}}^{t}+\varepsilon_{b} k_{z_{a}}^{i}},
\end{aligned}
$$

sendo $a, b=1,2$, sendo $a \neq b$.

Supondo a estrutura composta por três regiões, conforme ilustrado na 
Capítulo 4. Análise de estruturas planares a partir do método dos momentos MoM

Figura 4.8, faz-se a análise eletromagnética apresentada a seguir. Para a

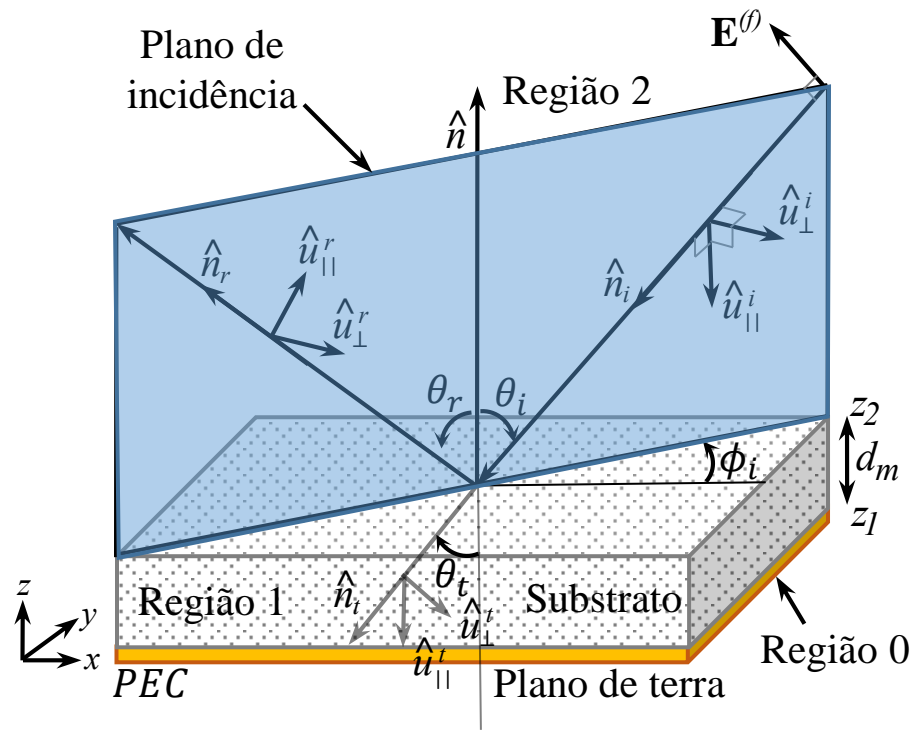

Figura 4.8: Reflexão no substrato e plano de terra.

polarização perperdicular do campo elétrico em relação ao plano de incidência, o campo elétrico total em cada região é descrito como sendo

$$
\begin{aligned}
& \mathbf{E}_{\perp_{2}}=E_{0_{\perp_{2}}}\left[\hat{u}_{\perp_{2}}^{i} e^{j k_{z_{2}} z}+\hat{u}_{\perp_{2}}^{r} R_{21}^{\perp} e^{j k_{z_{2}}\left(2 z_{2}-z\right)}\right] e^{j k_{x_{2}} x} e^{j k_{y_{2}} y} \\
& \mathbf{E}_{\perp_{1}}=E_{0_{\perp_{1}}}\left[\hat{u}_{\perp_{1}}^{i} e^{j k_{z_{1}} z}+\hat{u}_{\perp_{1}}^{r} \Gamma_{10}^{\perp} e^{j k_{z_{1}}\left(2 z_{1}-z\right)}\right] e^{j k_{x_{1}} x} e^{j k_{y_{1}} y}
\end{aligned}
$$

$\mathrm{e}$

$$
\mathbf{E}_{\perp_{0}}=\mathbf{0}
$$

e para a polarização paralela do campo elétrico

$$
\begin{gathered}
\mathbf{E}_{\|_{2}}=E_{0_{\|_{2}}}\left[\hat{u}_{\|_{2}}^{i} e^{j k_{z_{2}} z}+\hat{u}_{\|_{2}}^{r} R_{21}^{\|} e^{j k_{z_{2}}\left(2 z_{2}-z\right)}\right] e^{j k_{x_{2}} x} e^{j k_{y_{2}} y}, \\
\mathbf{E}_{\|_{1}}=E_{0_{\|_{1}}}\left[\hat{u}_{\|_{1}}^{i} e^{j k_{z_{1}} z}+\hat{u}_{\|_{1}}^{r} \Gamma_{10}^{\|} e^{j k_{z_{1}}\left(2 z_{1}-z\right)}\right] e^{j k_{x_{1}} x} e^{j k_{y_{1}} y}
\end{gathered}
$$

$\mathrm{e}$

$$
\mathbf{E}_{\|_{0}}=\mathbf{0} \quad \text { para } \quad z<0,
$$

em que o subíndice discrimina a região do espaço.

Os coeficientes de reflexão generalizados $\left(R^{\perp}\right.$ e $\left.R^{\|}\right)$e os termos de amplitudes $\left(E_{0_{\perp}}\right.$ e $\left.E_{0_{\|}}\right)$são obtidos através de

$$
R_{21}^{\xi}=\Gamma_{21}^{\xi}+\frac{T_{12}^{\xi} \Gamma_{10}^{\xi} T_{21}^{\xi} e^{j 2 k_{z_{1}}\left(z_{1}-z_{2}\right)}}{1-\Gamma_{12}^{\xi} \Gamma_{10}^{\xi} e^{j 2 k_{z_{1}}\left(z_{1}-z_{2}\right)}}=\Gamma_{21}^{\xi}+\frac{T_{12}^{\xi} \Gamma_{10}^{\xi} T_{21}^{\xi} e^{-j 2 k_{z_{1}} d_{m}}}{1-\Gamma_{12}^{\xi} \Gamma_{10}^{\xi} e^{-j 2 k_{z_{1}} d_{m}}}
$$

$\mathrm{e}$

$$
E_{0_{\xi_{1}}}=\frac{T_{12}^{\xi} E_{0_{\xi_{2}}} e^{j\left(k_{z_{2}}-k_{z_{1}}\right) z_{2}}}{1-\Gamma_{12}^{\xi} \Gamma_{10}^{\xi} e^{j 2 k_{z_{1}}\left(z_{1}-z_{2}\right)}}=\frac{T_{12}^{\xi} E_{0_{\xi_{2}}} e^{j\left(k_{z_{2}}-k_{z_{1}}\right) d_{m}}}{1-\Gamma_{12}^{\xi} \Gamma_{10}^{\xi} e^{-j 2 k_{z_{1}} d_{m}}}
$$


Capítulo 4. Análise de estruturas planares a partir do método dos momentos MoM

sendo $\xi=\perp$ ou $\|, z_{1}=0$ e $z_{2}=d_{m}$. Vale destacar que $\hat{u}_{\|_{1}}^{i}=\hat{u}_{\|_{2}}^{t}$ e $\hat{u}_{\|_{2}}^{t}=\hat{u}_{\|_{2}}^{r}$.

Adicionalmente, como o campo eletromagnético no interior da região 0 é zero, visto que corresponde a um material condutor perfeito, então $\Gamma_{10}^{\perp}=\Gamma_{10}^{\|}=-1$.

O campo elétrico total refletido para o espaço livre é descrito como segue,

$$
\mathbf{E}^{(r)}=\left[\hat{u}_{\perp_{2}}^{r} E_{0_{\perp_{2}}} R_{21}^{\perp}+\hat{u}_{\|_{2}}^{r} E_{0_{\|_{2}}} R_{21}^{\|}\right] e^{j k_{z_{0}}\left(2 d_{m}-z\right)} e^{j k_{x_{0}} x} e^{j k_{y_{0}} y},
$$

sendo o campo elétrico da fonte dado por

$$
\mathbf{E}^{(f)}=\left[\hat{u}_{\perp_{2}}^{i} E_{0_{\perp_{2}}}+\hat{u}_{\|_{2}}^{i} E_{0_{\|_{2}}}\right] e^{j k_{z_{0}} z} e^{j k_{x_{0}} x} e^{j k_{y_{0}} y} .
$$

O campo elétrico $\mathbf{E}^{(f)}$ pode ter componentes em $x$ e $y$, em caso de uma incidência normal e, $x, y$ e $z$ para o caso de uma incidência oblíqua, conforme pode ser visualizado na Figura 4.9. Entretanto, apenas é necessário avaliar os campos elétricos tangenciais à interface, neste caso nas direções $x$ e $y$, dados por

$$
E_{x}^{(f)}\left(x, y, z=d_{m}\right)=E_{0_{x}}^{i} e^{j\left(k_{0 x} x+k_{0} y\right)}
$$

$\mathrm{e}$

$$
E_{y}^{(f)}\left(x, y, z=d_{m}\right)=E_{0_{y}}^{i} e^{j\left(k_{0 x} x+k_{0 y} y\right)},
$$

sendo

$$
E_{0_{x}}^{i}=\left[E_{0_{\|_{2}}} \cos \left(\theta_{i}\right) \cos \left(\phi_{i}\right)+E_{0_{\perp_{2}}} \operatorname{sen}\left(\phi_{i}\right)\right] e^{j k_{0 z} d_{m}}
$$

e

$$
E_{0_{y}}^{i}=\left[E_{0_{\|_{2}}} \cos \left(\theta_{i}\right) \operatorname{sen}\left(\phi_{i}\right)-E_{0_{\perp_{2}}} \cos \left(\phi_{i}\right)\right] e^{j k_{0_{z}} d_{m}} .
$$

As mesmas considerações aplicadas na análise do campo da fonte sobre a metalização podem ser utilizadas para se determinar o campo refletido no substrato-GND e avaliado na interface,

$$
E_{x}^{(r)}\left(x, y, z=d_{m}\right)=E_{0_{x}}^{r} e^{j\left(k_{0 x} x+k_{0} y\right)}
$$

$\mathrm{e}$

$$
E_{y}^{(r)}\left(x, y, z=d_{m}\right)=E_{0_{y}}^{r} e^{j\left(k_{0 x} x+k_{0 y} y\right)},
$$

sendo

$$
E_{0_{x}}^{r}=\left[E_{0_{\|_{2}}} R_{21}^{\|} \cos \left(\theta_{i}\right) \cos \left(\phi_{i}\right)+E_{0_{\perp_{2}}} R_{21}^{\perp} \operatorname{sen}\left(\phi_{i}\right)\right] e^{j k_{0_{z}} d_{m}}
$$

e

$$
E_{0_{y}}^{r}=\left[E_{0_{\|_{2}}} R_{21}^{\|} \cos \left(\theta_{i}\right) \operatorname{sen}\left(\phi_{i}\right)-E_{0_{\perp_{2}}} R_{21}^{\perp} \cos \left(\phi_{i}\right)\right] e^{j k_{0_{z}} d_{m}}
$$

Aplica-se a transformada de Fourier das componentes tangenciais do campo elétrico refletido $\mathbf{E}^{(r)}$ na interface para se calcular o campo elétrico refletido no substrato em campo distante. Assim, no domínio espectral, tem-se que

$$
\tilde{\mathbf{E}}^{(r)}=\mathscr{F}\left(\mathbf{E}^{(r)}\right)=\hat{x} \mathscr{F}\left(E_{x}^{(r)}\right)+\hat{y} \mathscr{F}\left(E_{y}^{(r)}\right)=\hat{x} \tilde{E}_{x}^{(r)}+\hat{y} \tilde{E}_{y}^{(r)}
$$




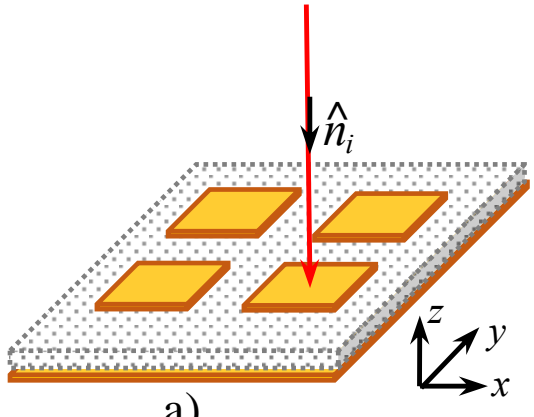

a)

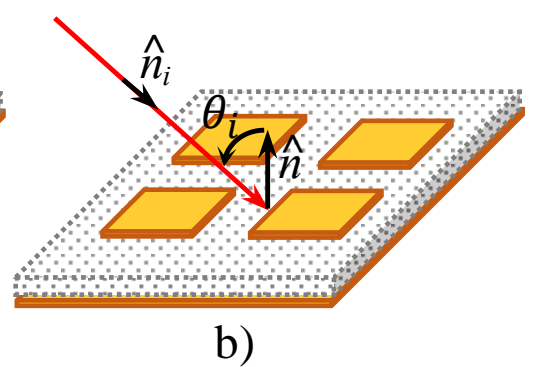

b)

Figura 4.9: Incidência sobre as metalizações. b) Normal; b) Oblíqua.

sendo

$$
\tilde{E}_{x}^{(r)}\left(k_{x}, k_{y}, z=d_{m}\right)=\int_{-\infty}^{+\infty} \int_{-\infty}^{+\infty} E_{x}^{(r)}\left(x, y, z=d_{m}\right) e^{-j\left(k_{x} x+k_{y} y\right)} d x d y
$$

$\mathrm{e}$

$$
\tilde{E}_{y}^{(r)}\left(k_{x}, k_{y}, z=d_{m}\right)=\int_{-\infty}^{+\infty} \int_{-\infty}^{+\infty} E_{y}^{(r)}\left(x, y, z=d_{m}\right) e^{-j\left(k_{x} x+k_{y} y\right)} d x d y .
$$

Após aplicada a transformada dupla de Fourier, cada componente do campo elétrico na interface é representada no domínio espectral por,

$$
\tilde{E}_{\zeta}^{(r)}=(2 \pi)^{2} \delta\left(k_{x}-k_{0_{x}}\right) \delta\left(k_{y}-k_{0_{y}}\right) E_{0_{\zeta}}^{r} \quad \text { com } \quad \zeta=x, y,
$$

em que $\delta($.$) denota a função delta de Dirac.$

Considerando que o substrato e o plano de terra apresentem dimensões finitas e posição central na origem do sistema de coordenadas, então o campo elétrico refletido sem a presença dos patches no domínio espectral é descrito por,

$$
\tilde{E}_{x}^{(r)}\left(k_{x}, k_{y}, z=d_{m}\right)=\int_{-B / 2}^{+B / 2} \int_{-A / 2}^{+A / 2} E_{x}^{(r)}\left(x, y, z=d_{m}\right) e^{-j\left(k_{x} x+k_{y} y\right)} d x d y
$$

$\mathrm{e}$

$$
\tilde{E}_{y}^{(r)}\left(k_{x}, k_{y}, z=d_{m}\right)=\int_{-B / 2}^{+B / 2} \int_{-A / 2}^{+A / 2} E_{y}^{(r)}\left(x, y, z=d_{m}\right) e^{-j\left(k_{x} x+k_{y} y\right)} d x d y,
$$

em que $A$ e $B$ são as dimensões do substrato e GND nas direções $x$ e $y$, respectivamente.

Após aplicada a transformada dupla de Fourier através da (4-157) e (4158), cada componente do campo elétrico refletido na interface sem a presença 
dos patches é representada por,

$$
\tilde{E}_{\zeta}^{(r)}=A B\left[\operatorname{sinc}\left(\frac{\left(k_{0_{x}}-k_{x}\right) A}{2}\right) \operatorname{sinc}\left(\frac{\left(k_{0_{y}}-k_{y}\right) B}{2}\right)\right] E_{0_{\zeta}}^{r},
$$

sendo $\zeta=x, y$ e $\operatorname{sinc}(\xi)=\operatorname{sen}(\xi) / \xi$.

Caso o alimentador possua componentes perpendicular e paralela (como é o caso de ondas polarizadas elipticamente, circularmente ou linear diagonal), então o princípio da lineariedade deve ser utilizado para computar as componentes do campo elétrico.

\section{3 \\ Campo elétrico difratado}

Diversas técnicas são propostas na literatura para representar os campos difratados por bordas condutoras. Algumas delas apresentam complexidade na formulação e facilidade de implementação, entretanto, o campo elétrico difratado não é possível ser representado em regiões de transição de reflexão e iluminação devido às singularidades (conhecidas como regiões de sombra), podendo-se citar técnicas da geometria óptica (do inglês, geometrical optics GO), tais como a teoria geométrica da difração (do inglês, geometrical theory of diffraction - GTD) e a teoria uniforme da difração (do inglês, uniform geometrical theory of diffraction - UTD), propostas em [92,93], da óptica física (do inglês, physical optics - PO), tais como o método das correntes de borda (do inglês, method of edge currents - MEC) [94-96], e da teoria física da difração (do inglês, physical theory of diffraction - PTD) [97,98].

Estas singularidades foram removidas em alguns trabalhos, podendo-se citar [99-103] para o método das correntes equivalentes de borda e [104] para a teoria física da difração. Em comparação, apesar de [104] apresentar a remoção das singularidades para os diversos casos críticos de incidência (onda rasante) e de observação, os campos difratados apenas podem ser avaliados em planos específicos, enquanto que em [103] a abordagem, além de ampla e clara, permite calcular o campo difratado em qualquer direção no espaço.

Neste trabalho optou-se em utilizar a formulação apresentada por [103], em que os problemas encontrados nas regiões de transição da GO são removidos a partir da separação do campo difratado em duas parcelas: a primeira consiste na componente de franja (não uniforme) definida por [98] e a segunda a componente uniforme, que representa as correntes da PO nas proximidades da borda. Assim, as singularidades são extraídas a partir do uso de coordenadas locais e correção da fase dos campos incidentes [103].

O campo difratado por uma das bordas do plano de terra é dado por 
Capítulo 4. Análise de estruturas planares a partir do método dos momentos MoM

$$
\mathbf{E}^{d}(\mathbf{s}, w)=\mathbf{E}^{F W C}(\mathbf{s}, w)+\mathbf{E}^{P O C}(\mathbf{s}, w)
$$

sendo

$$
\begin{aligned}
& \mathbf{E}^{F W C}(\mathbf{s}, w)=-\frac{1}{4 \pi r} \int_{C}\left\{\left[\left[\mathbf{E}^{(i n c)}\left(\mathbf{s}^{\prime}, w\right) \cdot \hat{t}\right] D_{e}^{I, f}\left(\beta_{0}^{\prime}, \beta_{0}, \phi_{0}^{\prime}, \phi_{0}\right)\right.\right. \\
& \left.+\left[\mathbf{H}^{(i n c)}\left(\mathbf{s}^{\prime}, w\right) \cdot \hat{t}\right] \eta_{0} D_{h}^{I, f}\left(\beta_{0}^{\prime}, \beta_{0}, \phi_{0}^{\prime}, \phi_{0}\right)\right][\hat{t}-(\hat{t} . \hat{s}) \hat{s}] \\
& \left.+\left[\mathbf{H}^{(i n c)}\left(\mathbf{s}^{\prime}, w\right) \cdot \hat{t}\right] \eta_{0} D_{h}^{M, f}\left(\beta_{0}^{\prime}, \beta_{0}, \phi_{0}^{\prime}, \phi_{0}\right)[\hat{t} \times \hat{s}]\right\} e^{-j k_{0}\left|\mathbf{s}-\mathbf{s}^{\prime}\right|} d l^{\prime}
\end{aligned}
$$

a parcela devido a corrente de franja e

$$
\begin{gathered}
\mathbf{E}^{P O C}(\mathbf{s}, w)=-\frac{1}{4 \pi r} \int_{C}\left\{\left[\left[\mathbf{E}^{(i n c)}\left(\mathbf{s}^{\prime}, w\right) \cdot \hat{t}\right] D_{e}^{I, P O}\left(\beta_{0}^{\prime}, \phi_{0}^{\prime}\right)\right.\right. \\
\left.+\left[\mathbf{H}^{(i n c)}\left(\mathbf{s}^{\prime}, w\right) \cdot \hat{t}\right] \eta_{0} D_{h}^{I, P O}\left(\beta_{0}^{\prime}, \beta_{0}, \phi_{0}^{\prime}, \phi_{0}\right)\right][\hat{t}-(\hat{t} . \hat{s}) \hat{s}] \\
\left.+\left[\mathbf{H}^{(i n c)}\left(\mathbf{s}^{\prime}, w\right) \cdot \hat{t}\right] \eta_{0} D_{h}^{M, P O}\left(\beta_{0}, \phi_{0}\right)[\hat{t} \times \hat{s}]\right\} F_{e c}(w) e^{-j k_{0}\left|\mathbf{s}-\mathbf{s}^{\prime}\right|} d l^{\prime}
\end{gathered}
$$

devido às correntes da $\mathrm{PO}$ na vizinhança da borda, em que a integral é realizada ao longo da borda da geometria. Os ângulos, dimensões e vetores envolvidos na formulação podem ser observados na Figura 4.10.

Para uma borda metálica com espessura de $34 \mu \mathrm{m}$ (dimensão comercial da metalização de laminados de microondas), tem-se que os coeficientes de difração usados no cálculo do campo elétrico difratado devido às correntes de franja são descritos por

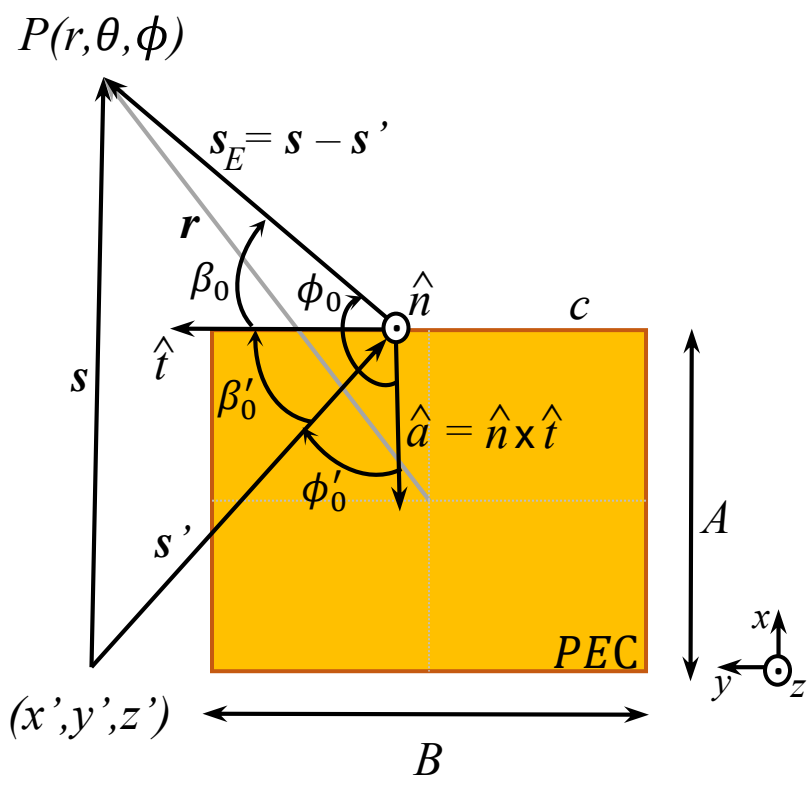

Figura 4.10: Difração na borda do plano de terra. 
Capítulo 4. Análise de estruturas planares a partir do método dos momentos -

$\mathrm{e}$

$$
\begin{aligned}
D_{h}^{I, f}\left(\beta_{0}^{\prime}, \beta_{0}, \phi_{0}^{\prime}, \phi_{0}\right)=\left\{\frac{\cot \left(\beta_{0}^{\prime}\right)\left[1+2 \operatorname{sen}\left(\alpha_{E} / 2\right) \cos \left(\phi_{0}^{\prime} / 2\right)\right]}{\operatorname{sen}\left(\beta_{0}^{\prime}\right) \operatorname{sen}\left(\alpha_{E} / 2\right)}\right. \\
\left.-\frac{\cot \left(\beta_{0}\right) \cos \left(\phi_{0}\right)}{\operatorname{sen}\left(\beta_{0}^{\prime}\right) \operatorname{sen}\left(\alpha_{E} / 2\right)}\right\} g\left(\phi_{0}^{\prime}, \alpha_{E}\right)
\end{aligned}
$$

$$
D_{e}^{I, f}\left(\beta_{0}^{\prime}, \beta_{0}, \phi_{0}^{\prime}, \phi_{0}\right)=\frac{\operatorname{sen}\left(\phi_{0}^{\prime} / 2\right)}{\operatorname{sen}^{2}\left(\beta_{0}^{\prime}\right)} g\left(\phi_{0}^{\prime}, \alpha_{E}\right)
$$

$$
D_{h}^{M, f}\left(\beta_{0}^{\prime}, \beta_{0}, \phi_{0}^{\prime}, \phi_{0}\right)=\frac{\operatorname{sen}\left(\phi_{0}\right)}{\operatorname{sen}\left(\beta_{0}\right) \operatorname{sen}\left(\beta_{0}^{\prime}\right) \operatorname{sen}\left(\alpha_{E} / 2\right)} g\left(\phi_{0}^{\prime}, \alpha_{E}\right),
$$

com

$$
g\left(\phi_{0}^{\prime}, \alpha_{E}\right)=\frac{1}{\cos \left(\phi_{0}^{\prime} / 2\right)+\operatorname{sen}\left(\alpha_{E} / 2\right)}
$$

$\mathrm{e}$

$$
\alpha_{E}=\cos ^{-1}\left[\frac{\operatorname{sen}\left(\beta_{0}\right) \cos \left(\phi_{0}\right)+\cot \left(\beta_{0}^{\prime}\right)\left[\cos \left(\beta_{0}\right)-\cos \left(\beta_{0}^{\prime}\right)\right]}{\operatorname{sen}\left(\beta_{0}^{\prime}\right)}\right] .
$$

Os coeficientes de difração para o campo elétrico difratado devido às correntes de borda da PO são descritos como sendo,

$$
\begin{gathered}
D_{h}^{M, P O}\left(\beta_{0}, \phi_{0}\right)=-\frac{2 \operatorname{sen}\left(\phi_{0}\right)}{\operatorname{sen}\left(\beta_{0}\right)}, \\
D_{e}^{I, P O}\left(\beta_{0}^{\prime}, \phi_{0}^{\prime}\right)=\frac{2 \operatorname{sen}\left(\phi_{0}^{\prime}\right)}{\operatorname{sen}\left(\beta_{0}^{\prime}\right)}
\end{gathered}
$$

$\mathrm{e}$

$$
D_{h}^{I, P O}\left(\beta_{0}^{\prime}, \beta_{0}, \phi_{0}^{\prime}, \phi_{0}\right)=-2\left[\cot \left(\beta_{0}^{\prime}\right) \cos \left(\phi_{0}^{\prime}\right)+\cot \left(\beta_{0}\right) \cos \left(\phi_{0}\right)\right] .
$$

Além disso, a função de transição $F_{e c}(w)$ da (4-162) é dada por,

$$
F_{e c}(w)=B_{1} \sqrt{\pi} \frac{e^{j\left(w B_{2}^{2}-\pi / 4\right)}}{\sqrt{w}} \operatorname{erfc}\left(B_{2} \sqrt{j w}\right),
$$

sendo erfc(.) a função erro complementar, que é calculada a partir de

$$
\operatorname{erfc}\left(B_{2} \sqrt{j w}\right)=\frac{2}{\sqrt{\pi}} \int_{B_{2} \sqrt{j w}}^{\infty} e^{-t^{2}} d t
$$

com

$$
\begin{gathered}
B_{1}=-\operatorname{sgn}\left(A_{1}\right) \sqrt{\frac{c}{A_{2}}}, \\
B_{2}=\frac{\left|A_{1}\right|}{\sqrt{c A_{2}}}, \\
A_{1}=\cos (\gamma)-\cos \left(\gamma^{\prime}\right)
\end{gathered}
$$

$\mathrm{e}$

$$
A_{2}=2\left(\frac{\operatorname{sen}\left(\beta_{0}\right) \operatorname{sen}\left(\phi_{0}\right)+\operatorname{sen}\left(\beta_{0}^{\prime}\right) \operatorname{sen}\left(\phi_{0}^{\prime}\right)}{R_{c b}}+\frac{\operatorname{sen}^{2}(\gamma)}{s_{E}}+\frac{\operatorname{sen}^{2}\left(\gamma^{\prime}\right)}{s^{\prime}}\right),
$$


Capítulo 4. Análise de estruturas planares a partir do método dos momentos MoM

em que $\operatorname{sgn}($.$) é a função sinal, R_{c b}$ é o raio de curvatura da borda (para a borda reta $\left.R_{c b} \rightarrow \infty\right)$ e $s_{E}$ é a distância do ponto de difração até o ponto de observação.

O cálculo destes parâmetros depende dos ângulos entre os vetores relacionados, que conforme apresentados na Figura 4.10, são dados por

$$
\begin{gathered}
\cos \left(\beta_{0}^{\prime}\right)=\frac{\mathbf{s}^{\prime}}{\left|\mathbf{s}^{\prime}\right|} \cdot \hat{t}, \\
\cos \left(\beta_{0}\right)=\frac{\mathbf{s}}{|\mathbf{s}|} \cdot \hat{t}, \\
\cos \left(\phi_{0}^{\prime}\right)=\frac{-\mathbf{s}^{\prime} . \hat{a}}{\left|\mathbf{s}^{\prime}\right| \operatorname{sen}\left(\beta_{0}^{\prime}\right)}, \\
\operatorname{sen}\left(\phi_{0}^{\prime}\right)=\frac{-\mathbf{s}^{\prime} \cdot \hat{n}}{\left|\mathbf{s}^{\prime}\right| \operatorname{sen}\left(\beta_{0}^{\prime}\right)}, \\
\cos \left(\phi_{0}\right)=\frac{\mathbf{s} \cdot \hat{a}}{|\mathbf{s}| \operatorname{sen}\left(\beta_{0}\right)}, \\
\operatorname{sen}\left(\phi_{0}\right)=\frac{\mathbf{s} \cdot \hat{n}}{|\mathbf{s}| \operatorname{sen}\left(\beta_{0}\right)}, \\
\cos (\gamma)=\cos \left(\beta_{0}\right)+\operatorname{sen}\left(\beta_{0}\right) \cos \left(\phi_{0}\right) \\
\cos \left(\gamma^{\prime}\right)=\cos \left(\beta_{0}^{\prime}\right)-\operatorname{sen}\left(\beta_{0}^{\prime}\right) \cos \left(\phi_{0}^{\prime}\right),
\end{gathered}
$$

$\mathrm{e}$

em que $\hat{t}$ é um versor tangente à borda, $\hat{a}$ é um versor tangente ao plano de terra e $\hat{n}$ é um versor normal à borda.

A posição do ponto de observação é calculado em coordenadas cartesianas através de

$$
\hat{r}=\hat{x} \operatorname{sen}(\theta) \cos (\phi)+\hat{y} \operatorname{sen}(\theta) \operatorname{sen}(\phi)+\hat{z} \cos (\theta),
$$

sendo $\mathbf{r}=r \hat{r}$. Vale destacar que o campo difratado total corresponde ao somatório do campo difratado pelas quatro bordas do plano de terra.

\section{4}

\section{Campo elétrico irradiado}

Algumas técnicas são apresentadas em [105] para o cálculo do campo elétrico irradiado por uma superfície metálica de uma RA, tais como avaliação de fase estacionária da função diádica de Green, correntes equivalentes a partir do espectro de Floquet e correntes equivalentes a partir do espectro contínuo. Neste trabalho, determina-se o campo elétrico espalhado mais o refletido no hemifério superior $(z>0)$ utilizando a avaliação assintótica de integrais com 
aproximação de fase estacionária [6], descrito como

$$
\begin{aligned}
\mathbf{E}(r, \theta, \phi)= & j k_{0} \frac{e^{-j k_{0} r}}{2 \pi r}\left[(\hat{\theta} \cos \phi-\hat{\phi} \operatorname{sen} \phi \cos \theta)\left(\tilde{E}_{x}^{(e s p)}\left(k_{u}, k_{v}\right)+\tilde{E}_{x}^{(r)}\left(k_{u}, k_{v}\right)\right)\right. \\
& \left.+(\hat{\theta} \operatorname{sen} \phi+\hat{\phi} \cos \phi \cos \theta)\left(\tilde{E}_{y}^{(e s p)}\left(k_{u}, k_{v}\right)+\tilde{E}_{y}^{(r)}\left(k_{u}, k_{v}\right)\right)\right],
\end{aligned}
$$

sendo $\tilde{E}_{x}^{(e s p)}$ e $\tilde{E}_{y}^{(e s p)}$ determinados a partir da (4-23) e (4-24), com $\tilde{J}_{x}\left(k_{u}, k_{v}\right)$ e $\tilde{J}_{y}\left(k_{u}, k_{v}\right)$ calculados através da $(4-10)$ e $(4-11)$, e $\tilde{E}_{x}^{(r)}$ e $\tilde{E}_{y}^{(r)}$ determinados a partir da (4-157) e (4-158). Neste caso, $k_{x}=k_{u}=k_{0} \operatorname{sen} \theta \cos \phi, k_{y}=k_{v}=$ $k_{0} \operatorname{sen} \theta \operatorname{sen} \phi, \beta^{2}=k_{u}^{2}+k_{v}^{2}$ e $\beta=k_{0} \operatorname{sen} \theta$.

A equação (4-186) pode ser reescrita da seguinte forma,

$$
\mathbf{E}(r, \theta, \phi)=j k_{0} \frac{e^{-j k_{0} r}}{2 \pi r}\left[\hat{\theta} E_{\theta}(\theta, \phi)+\hat{\phi} E_{\phi}(\theta, \phi)\right]
$$

sendo

$$
E_{\theta}(\theta, \phi)=\left[\tilde{E}_{x}^{(T)}\left(k_{u}, k_{v}\right)\right] \cos \phi+\left[\tilde{E}_{y}^{(T)}\left(k_{u}, k_{v}\right)\right] \operatorname{sen} \phi
$$

e

$$
E_{\phi}(\theta, \phi)=\left[-\tilde{E}_{x}^{(T)}\left(k_{u}, k_{v}\right) \operatorname{sen} \phi+\tilde{E}_{y}^{(T)}\left(k_{u}, k_{v}\right) \cos \phi\right] \cos \theta
$$

com

$$
\tilde{E}_{x}^{(T)}\left(k_{u}, k_{v}\right)=\left[\tilde{E}_{x}^{(r)}\left(k_{u}, k_{v}\right)+\tilde{E}_{x}^{(e s p)}\left(k_{u}, k_{v}\right)\right]
$$

e

$$
\tilde{E}_{y}^{(T)}\left(k_{u}, k_{v}\right)=\left[\tilde{E}_{y}^{(r)}\left(k_{u}, k_{v}\right)+\tilde{E}_{y}^{(e s p)}\left(k_{u}, k_{v}\right)\right] .
$$

Para uma rede composta por $E$ elementos espalhadores, (4-186) deve ser reescrita da seguinte forma,

$$
\begin{aligned}
& \mathbf{E}(r, \theta, \phi)=j k_{0} \frac{e^{-j k_{0} r}}{2 \pi r} {\left[\hat{\theta} E_{\theta}^{(r)}(\theta, \phi)+\hat{\phi} E_{\phi}^{(r)}(\theta, \phi)\right.} \\
&\left.+\sum_{q=1}^{E}\left[\hat{\theta} E_{\theta}^{q}(\theta, \phi)+\hat{\phi} E_{\phi}^{q}(\theta, \phi)\right]\right],
\end{aligned}
$$

sendo

$$
E_{\theta}^{q}(\theta, \phi)=\left[\tilde{E}_{x}^{q(e s p)}\left(k_{u}, k_{v}\right) \cos \phi+\tilde{E}_{y}^{q(e s p)}\left(k_{u}, k_{v}\right) \operatorname{sen} \phi\right]
$$

e

$$
E_{\phi}^{q}(\theta, \phi)=\left[-\tilde{E}_{x}^{q(e s p)}\left(k_{u}, k_{v}\right) \operatorname{sen} \phi+\tilde{E}_{y}^{q(e s p)}\left(k_{u}, k_{v}\right) \cos \phi\right] \cos \theta,
$$

que corresponde ao somatório vetorial do campo elétrico espalhado por cada elemento que compõe a rede refletora, com

$$
E_{\theta}^{(r)}(\theta, \phi)=\left[\tilde{E}_{x}^{(r)}\left(k_{u}, k_{v}\right)\right] \cos \phi+\left[\tilde{E}_{y}^{(r)}\left(k_{u}, k_{v}\right)\right] \operatorname{sen} \phi
$$

e

$$
E_{\phi}^{(r)}(\theta, \phi)=\left[-\tilde{E}_{x}^{(r)}\left(k_{u}, k_{v}\right) \operatorname{sen} \phi+\tilde{E}_{y}^{(r)}\left(k_{u}, k_{v}\right) \cos \phi\right] \cos \theta
$$


Quando o conceito de célula for introduzido mais adiante, o campo refletido será calculado sobre a área do plano de terra da célula e adicionado por superposição com o espalhado dentro do somatório da (4-192) para determinar o campo elétrico total.

\section{5}

\section{Validações numéricas}

Na validação numérica considerou-se as incidências normal e oblíqua de uma onda plana linearmente polarizada, com orientação do vetor campo elétrico em $x$ (transversal eletromagnética - TEM), sobre uma metalização que possui as seguintes dimensões físicas aproximadas na frequência de ressonância $\left(f_{r}=2,4 \mathrm{GHz}\right): L=W=34 \mathrm{~mm}$. A estrutura abaixo do patch é semelhante a apresentada na Figura 3.6, com substrato de espessura $d_{m}=1,524 \mathrm{~mm}$ e permissividade relativa do substrato $\varepsilon_{r_{b}}=3,38$. Os pesos dos modos assumidos para a densidade de corrente superficial sobre a metalização foram determinados a partir do método dos momentos utilizando as seguintes funções base de domínio completo: senoidal, senoidal com condição de borda sem segmentação e senoidal com condição de borda segmentada $(0,2 \leq \kappa \leq 0,8)$. As análises foram realizadas assumindo-se apenas um modo em $x$ e $y$. A integral numérica no terceiro intervalo foi limitada em $100 k_{0}(\mathscr{P}=100)$. O valor de afastamento do caminho de integração em relação ao pólo foi setado em $\delta=k_{0} \times 10^{-3} \mathrm{e}$ para a derivada numérica $\Delta \beta_{\xi}=k_{0} \times 10^{-13}$. Posteriormente, os campos elétricos espalhado e refletido foram determinados a partir do método da fase estacionária utilizando as correntes calculadas e a função diádica de Green. Os resultados foram comparados com os obtidos a partir do Ansys Designer e HFSS.

O Designer foi utilizado para a verificação dos campos espalhados para elementos simples e redes refletoras, visto que o software é baseado na solução do problema a partir do método dos momentos com plano de terra e substrato infinitos, o que, para este caso, é compatível com a modelagem da função de Green aqui descrita. Adicionalmente, para uma simulação de onda completa, o software Designer não considera a reflexão, ou seja, o campo incidente no patch é igual ao campo da fonte. Para fins de comparação, considera-se $\mathbf{E}^{(i n c)}\left(\mathbf{J}^{(f)}\right)=\mathbf{E}^{(f)}\left(\mathbf{J}^{(f)}\right)$, sendo $\mathbf{E}^{(r)}\left(\mathbf{J}^{(f)}\right)=\mathbf{0}$.

Os efeitos da reflexão no substrato e plano de terra são verificados a partir do software Ansys HFSS, que resolve numericamente a estrutura através do método dos elementos finitos e permite a inclusão da refração no dielétrico e a difração de borda. Neste método considera-se que as dimensões do plano de terra e substrato são finitas. Por fim, o campo difratado é incluído juntamente 
Capítulo 4. Análise de estruturas planares a partir do método dos momentos MoM

com os campos espalhado e refletido para representar o campo elétrico total re-irradiado pela estrutura.

\subsection{1}

\section{Campo elétrico espalhado por um elemento simples}

Na Figura 4.11 é ilustrado o elemento espalhador metálico que é iluminado por uma onda plana com incidência normal.

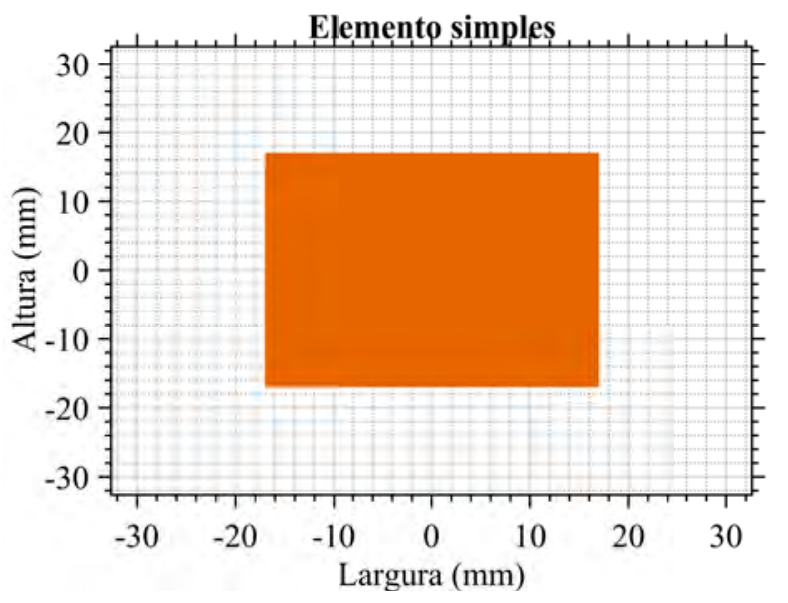

Figura 4.11: Elemento simples.

As densidades de corrente superficial ao longo de $x$ e $y$ para funções base senoidais podem ser visualizadas na Figura 4.12 e o campo elétrico espalhado nos planos de elevação e de azimute na Figura 4.13.

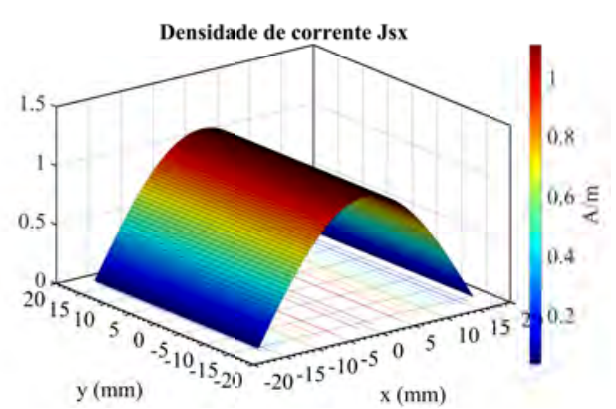

a)

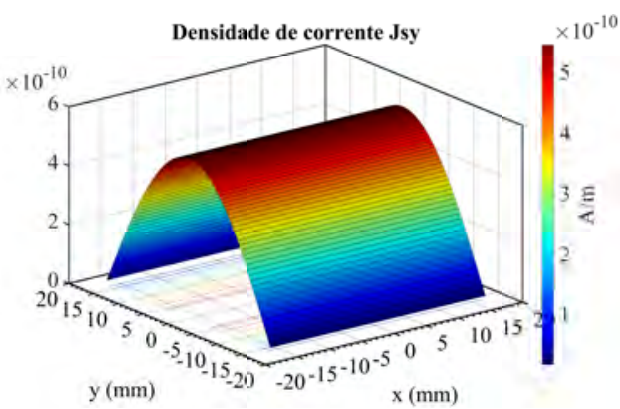

b)

Figura 4.12: Densidade de corrente superficial para funções base senoidal. a) $J_{x}(x, y)$; b) $J_{y}(x, y)$. 


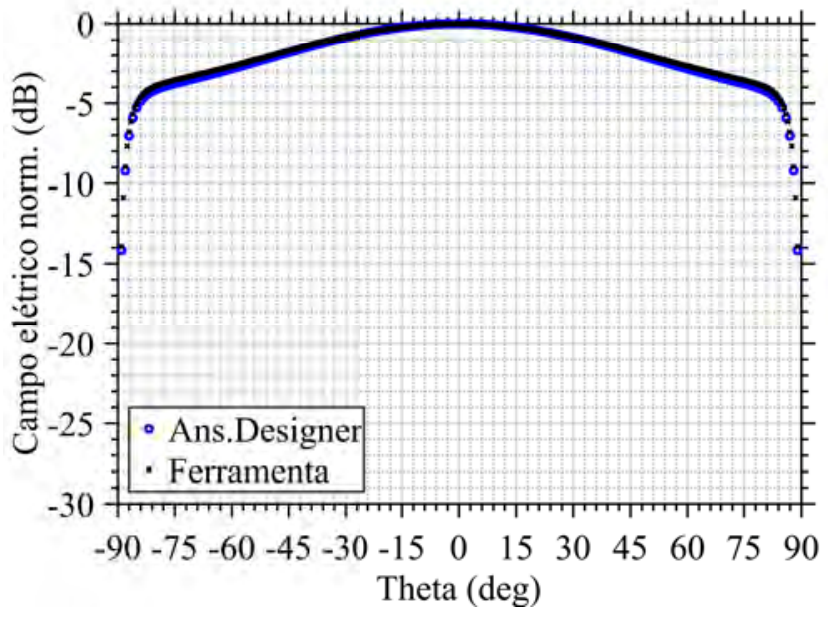

a)

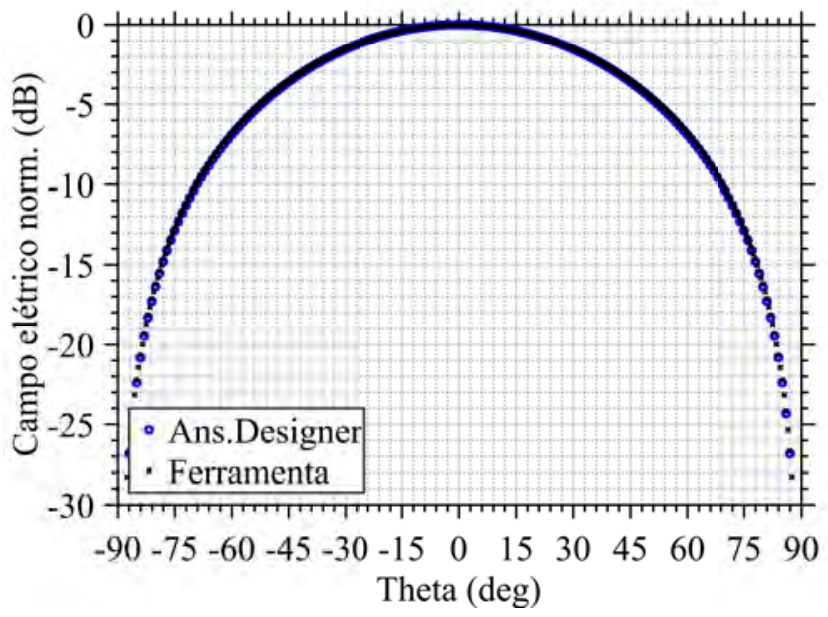

b)

Figura 4.13: Campo calculado e simulado para funções base senoidal. a) Plano E: $E_{\theta}\left(\theta, \phi=0^{\circ}\right)$; b) Plano $\mathrm{H}: E_{\phi}\left(\theta, \phi=90^{\circ}\right)$.

As densidades de corrente superficial ao longo de $x$ e $y$ para funções base com condição de borda sem segmentação podem ser visualizadas na Figura 4.14 e o campo elétrico espalhado na Figura 4.15.

Por fim, as densidades de corrente superficial ao longo de $x$ e $y$ para funções base com condição de borda segmentada $(\kappa=0,4)$ podem ser visualizadas na Figura 4.16 e o campo elétrico espalhado na Figura 4.17. O comportamento para outras segmentações é apresentado no apêndice B. 
Capítulo 4. Análise de estruturas planares a partir do método dos momentos MoM

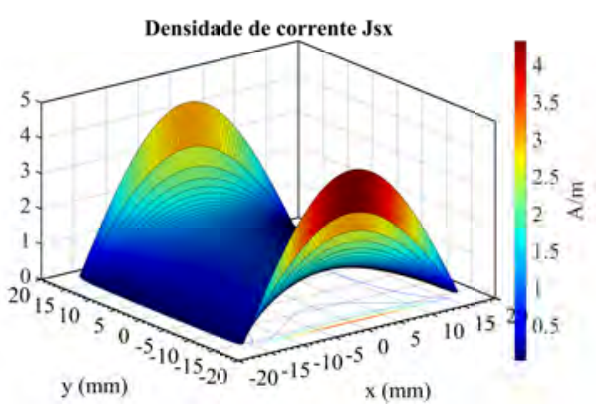

a)

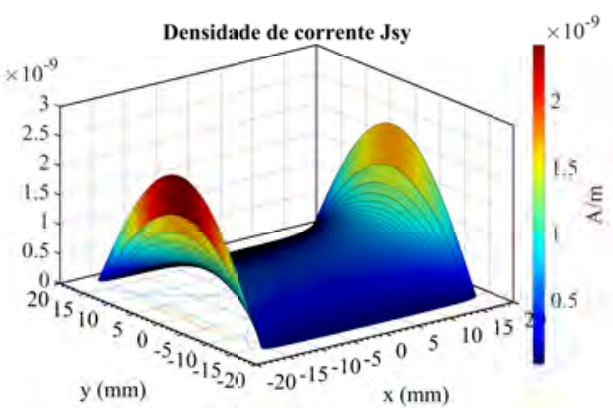

b)

Figura 4.14: Densidade de corrente superficial para funções base com condição de borda sem segmentação. a) $J_{x}(x, y)$; b) $J_{y}(x, y)$.

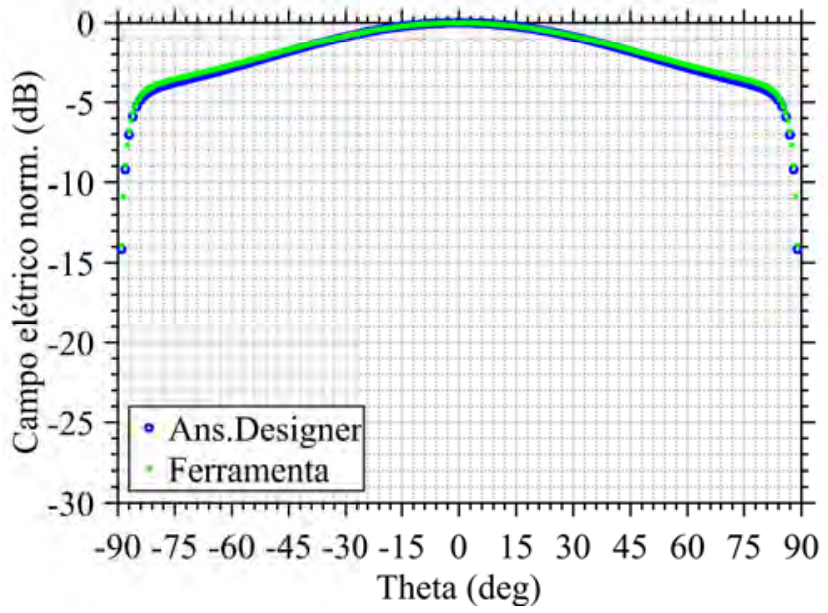

a)

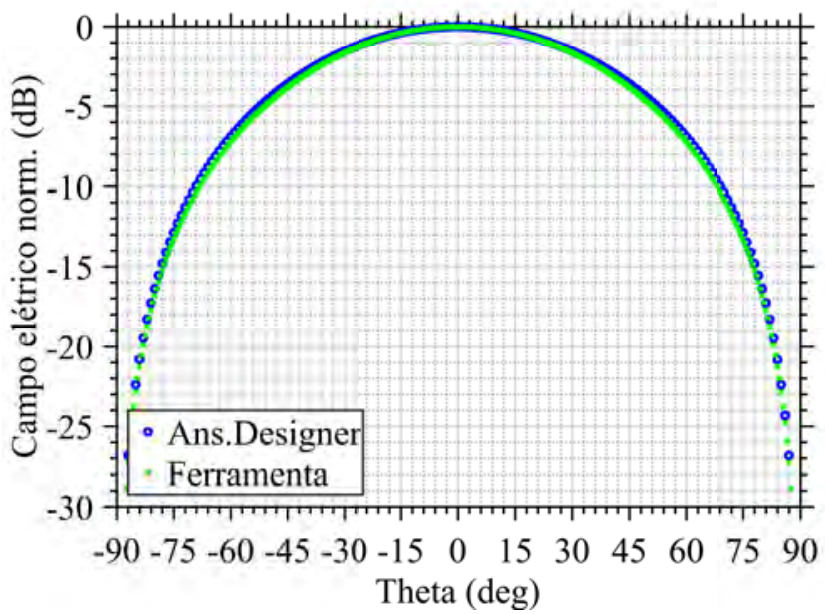

b)

Figura 4.15: Campo calculado e simulado para funções base com condição de borda sem segmentação. a) Plano E: $E_{\theta}\left(\theta, \phi=0^{\circ}\right)$; b) Plano $\mathrm{H}: E_{\phi}\left(\theta, \phi=90^{\circ}\right)$. 
Capítulo 4. Análise de estruturas planares a partir do método dos momentos MoM

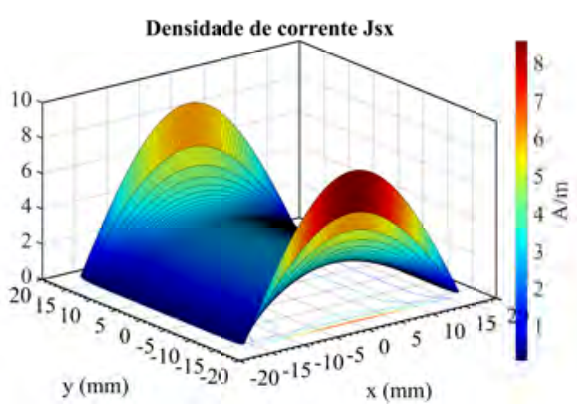

a)

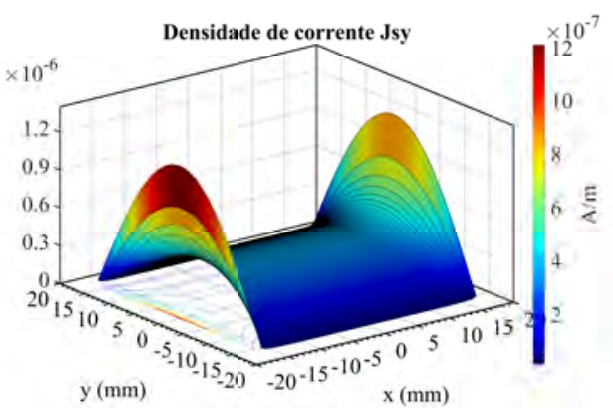

b)

Figura 4.16: Densidade de corrente superficial para funções base com condição de borda segmentada, $\kappa=0,4$. a) $J_{x}(x, y)$; b) $J_{y}(x, y)$.

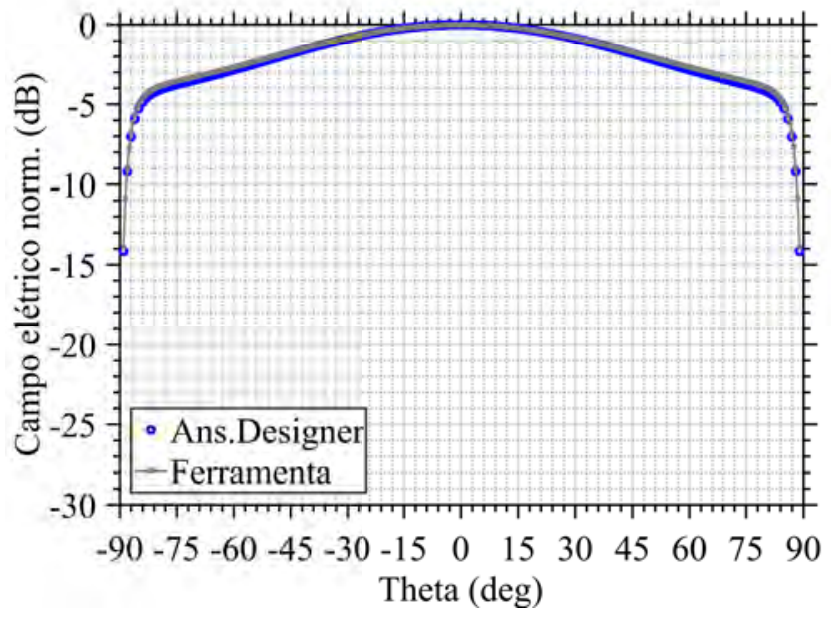

a)

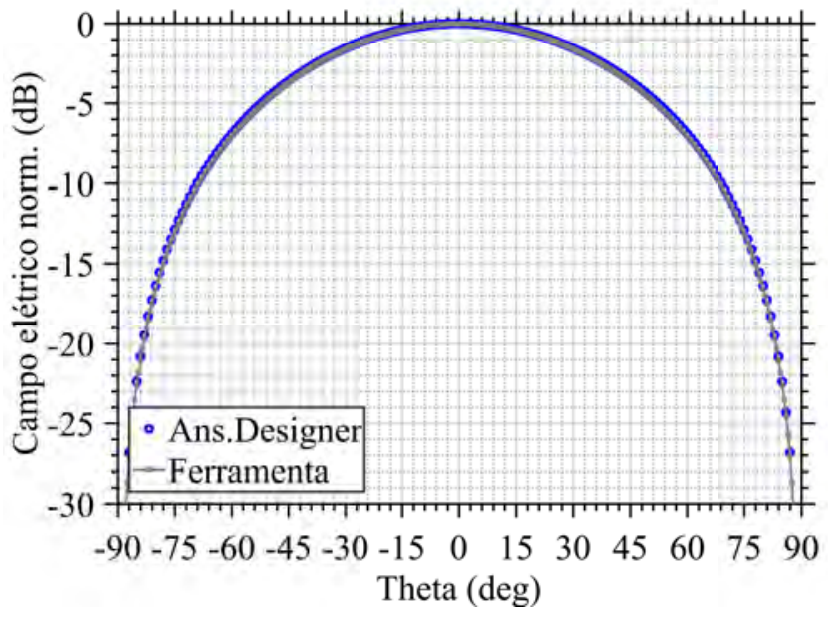

b)

Figura 4.17: Campo calculado e simulado para funções base com condição de borda segmentada, $\kappa=0$, 4. a) Plano $\mathrm{E}$ : $E_{\theta}\left(\theta, \phi=0^{\circ}\right)$; b) Plano $\mathrm{H}$ : $E_{\phi}\left(\theta, \phi=90^{\circ}\right)$. 
Capítulo 4. Análise de estruturas planares a partir do método dos momentos MoM

Percebe-se que todos os diagramas de irradiação para as diferentes funções base analisadas apresentam excelente concordância com o simulador comercial. Entretanto, também deve ser realizada a variação das dimensões $L$ e $W$ para obtenção das curvas de fase e amplitude do campo elétrico espalhado pelo elemento. As curvas são extraídas para as funções base abordadas neste trabalho e os resultados obtidos podem ser visualizados nas Figuras 4.18 e 4.19 .

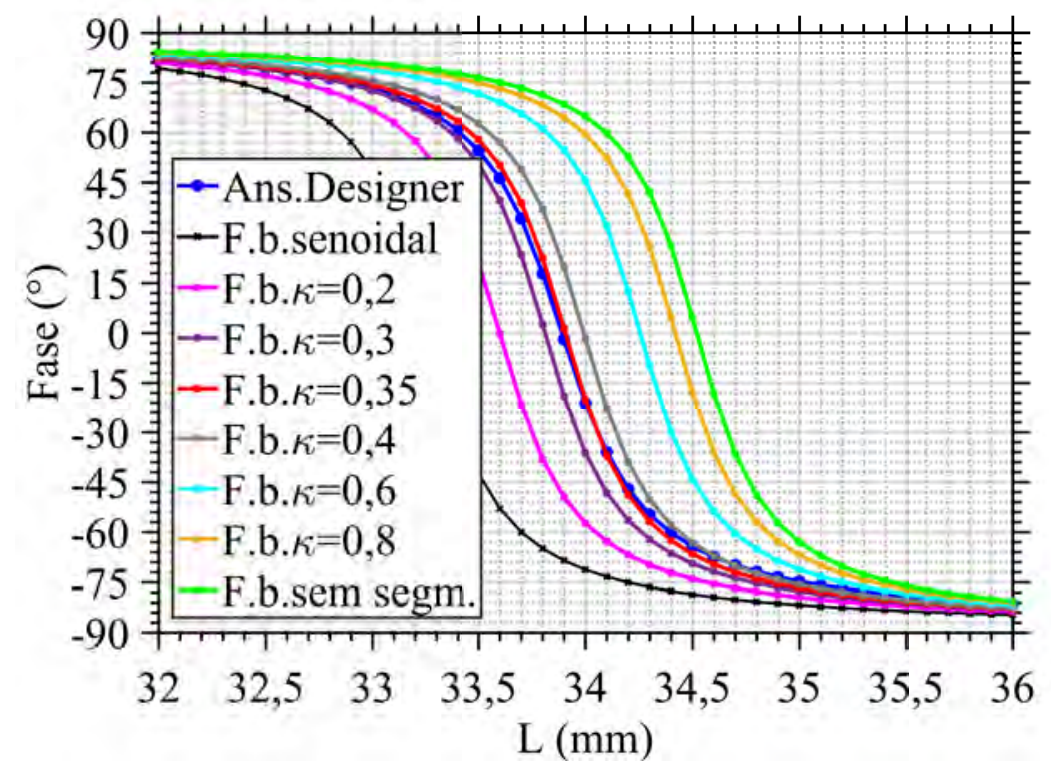

Figura 4.18: Curvas de fase para as diferentes funções base com $W=L$.

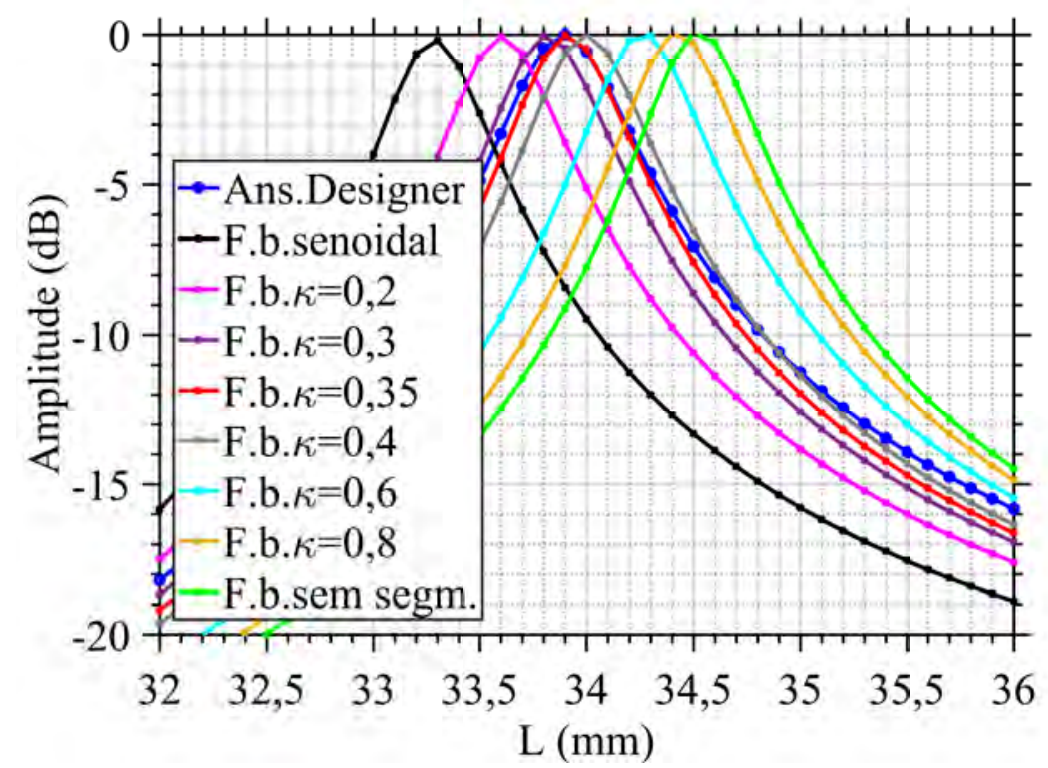

Figura 4.19: Curvas de amplitude para as diferentes funções base com $W=L$. 
Percebe-se que as curvas de fase e amplitude divergem com o software de verificação para o número de funções base senoidais e com condição de borda sem segmentação utilizadas na solução. Entretanto, aplicando corretamente a função base com condição de borda segmentada $(\kappa \sim 0,35)$, verifica-se excelente concordância com o software Ansys Designer. O erro máximo para a fase é de aproximadamente $\pm 5^{\circ}$ e para a amplitude de aproximadamente $0,8 \mathrm{~dB}$, respectivamente.

Na Figura 4.19 percebe-se que a máxima irradiação ocorre para a dimensão em que estrutura ressoa, ou seja, quando o patch possui a dimensão de aproximadamente $34 \mathrm{~mm}$. Para substratos finos em relação ao comprimento de onda, essa largura é aproximadamente determinada como sendo,

$$
L=\frac{c}{2 f_{r} \sqrt{\varepsilon_{r_{b}}}},
$$

em que $c$ é a velocidade da luz no vácuo e $f_{r}$ é a frequência de ressonância (neste caso, 2,4 GHz). O projeto de RAs com o ajuste de fase a partir das dimensões das metalizações impressas requer a aplicação de substratos finos para o aumento da faixa de variação de fase. Então (4-197), juntamente com a curva de amplitude, podem ser utilizadas para ajustar adequadamente o parâmetro $\kappa$.

\subsection{2}

\section{Campo elétrico espalhado por redes refletoras}

Após determinado o comportamento da densidade de corrente superficial sobre um patch, verifica-se o campo elétrico espalhado por redes refletoras a partir do método dos momentos com função de borda segmentada $(\kappa=0,35)$. A estrutura analisada possui patches de mesmo tamanho físico, sendo $W=L=34 \mathrm{~mm}$ e espaçamento centro-a-centro de $\lambda_{0} / 2$.

As antenas são iluminadas por uma onda plana com incidências normal e oblíqua. Redes refletoras de dimensão $3 \times 3,5 \times 5$ e $7 \times 7$ são apresentadas nas Figuras 4.20, 4.21 e 4.22 e analisadas através do software comercial Ansys Designer e pela ferramenta proposta neste trabalho. Os campos espalhados obtidos para inclinações do campo elétrico incidente de $0^{\circ}, 10^{\circ}$ e $20^{\circ}$ são ilustrados nas Figuras 4.23 a 4.25, Figuras 4.26 a 4.28 e Figuras 4.29 a 4.31 . 
Capítulo 4. Análise de estruturas planares a partir do método dos momentos MoM

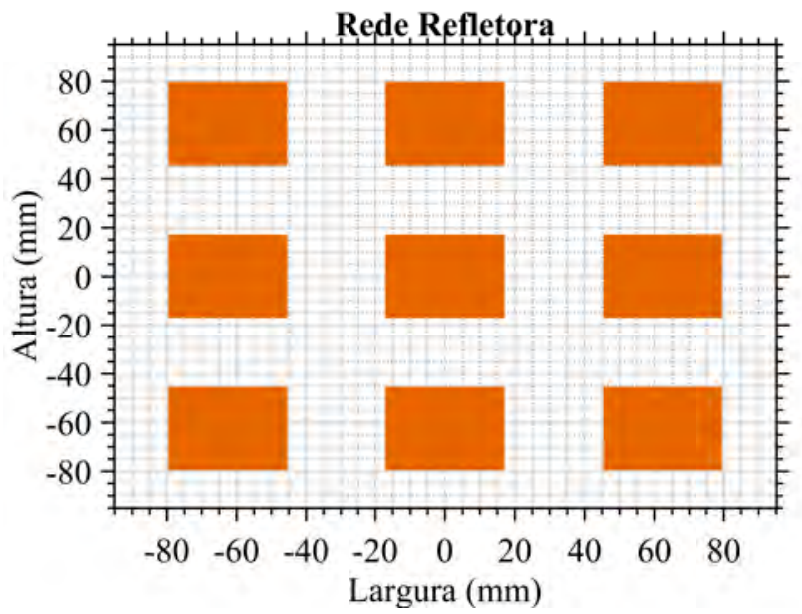

Figura 4.20: Rede $3 \times 3$ com elementos de mesma dimensão física.

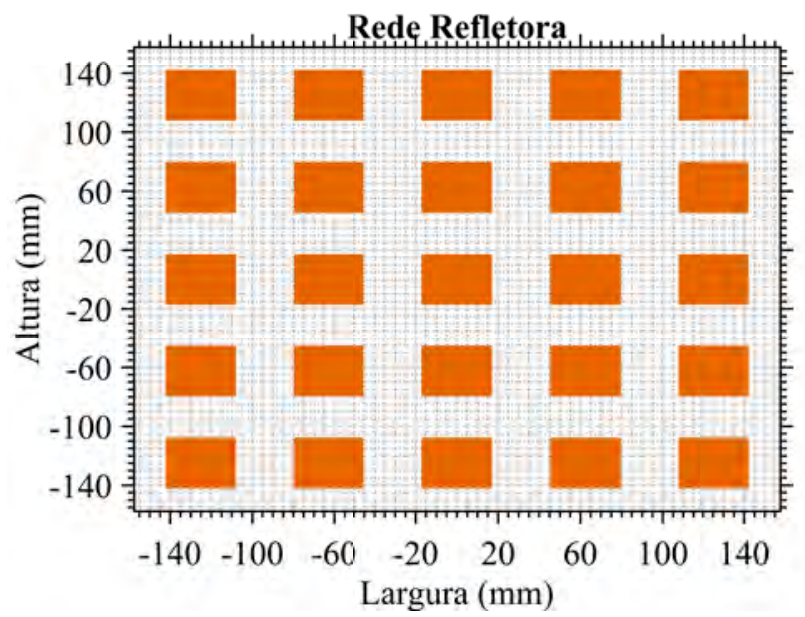

Figura 4.21: Rede $5 \times 5$ com elementos de mesma dimensão física.

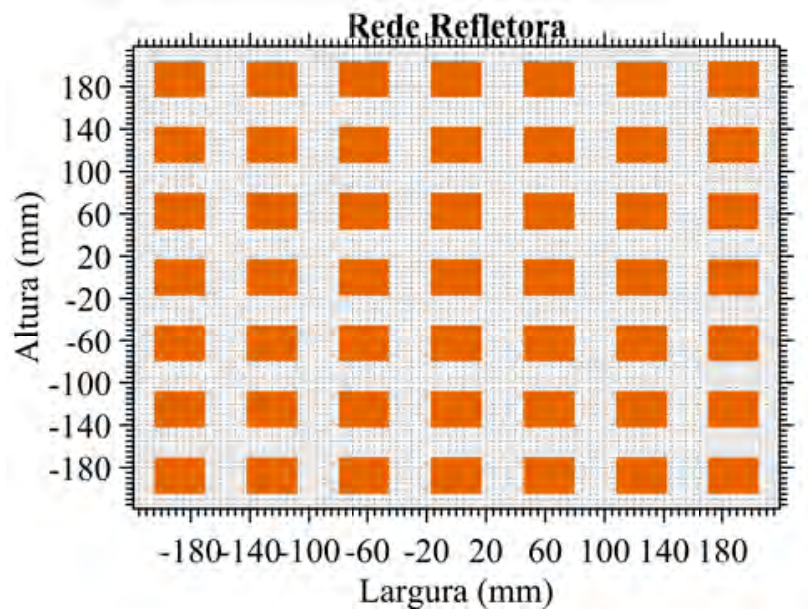

Figura 4.22: Rede $7 \times 7$ com elementos de mesma dimensão física. 
Capítulo 4. Análise de estruturas planares a partir do método dos momentos MoM

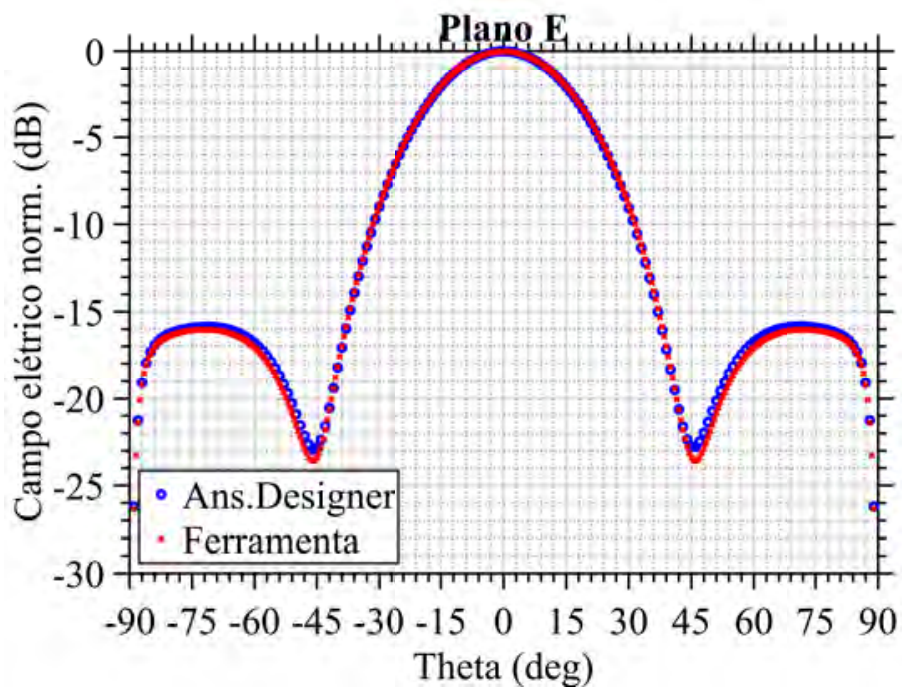

a)

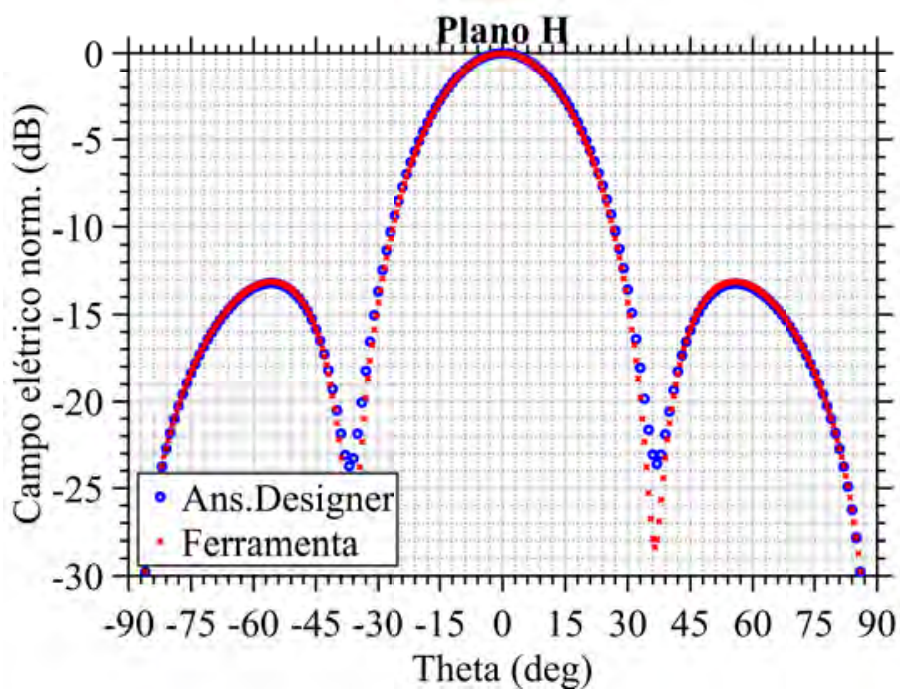

b)

Figura 4.23: Campo calculado e simulado para uma rede $3 \times 3$ - onda plana com incidência normal. a) Plano E: $E_{\theta}\left(\theta, \phi=0^{\circ}\right)$; b) Plano $\mathrm{H}: E_{\phi}\left(\theta, \phi=90^{\circ}\right)$. 
Capítulo 4. Análise de estruturas planares a partir do método dos momentos MoM

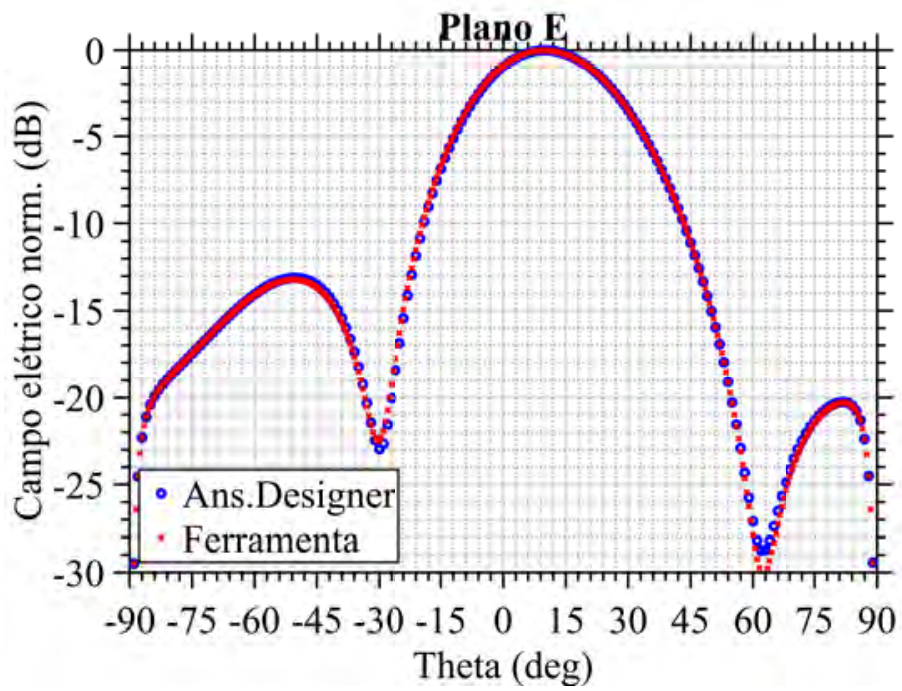

Figura 4.24: Campo calculado e simulado para uma rede $3 \times 3$ - onda plana com incidência oblíqua $\left(\theta_{i}=10^{\circ}\right)$. Plano E: $E_{\theta}\left(\theta, \phi=0^{\circ}\right)$.

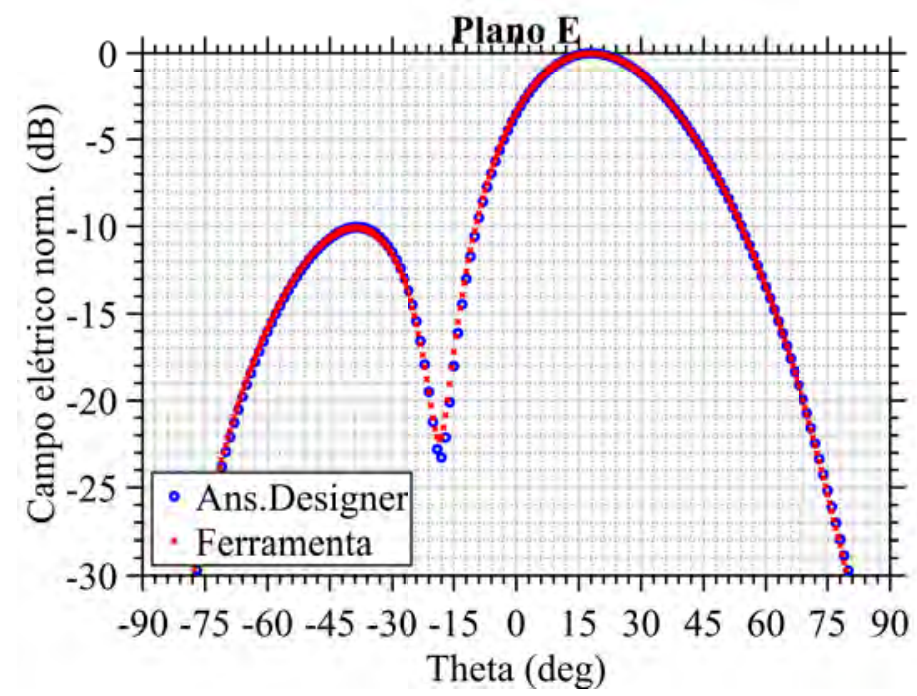

Figura 4.25: Campo calculado e simulado para uma rede $3 \times 3$ - onda plana com incidência oblíqua $\left(\theta_{i}=20^{\circ}\right)$. Plano E: $E_{\theta}\left(\theta, \phi=0^{\circ}\right)$. 
Capítulo 4. Análise de estruturas planares a partir do método dos momentos MoM

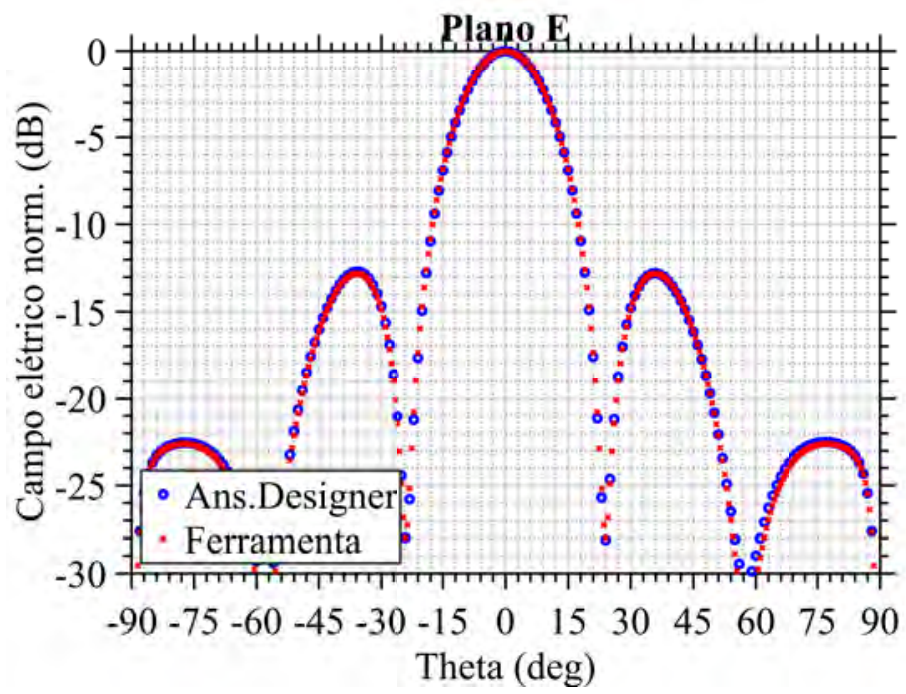

a)

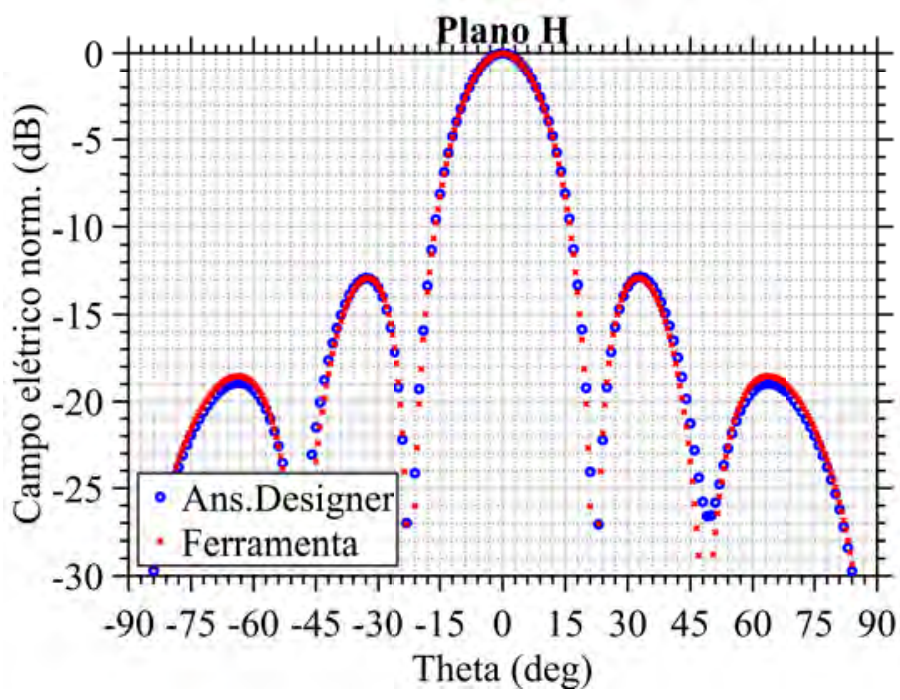

b)

Figura 4.26: Campo calculado e simulado para uma rede $5 \times 5$ - onda plana com incidência normal. a) Plano E: $E_{\theta}\left(\theta, \phi=0^{\circ}\right)$; b) Plano $\mathrm{H}: E_{\phi}\left(\theta, \phi=90^{\circ}\right)$. 
Capítulo 4. Análise de estruturas planares a partir do método dos momentos MoM

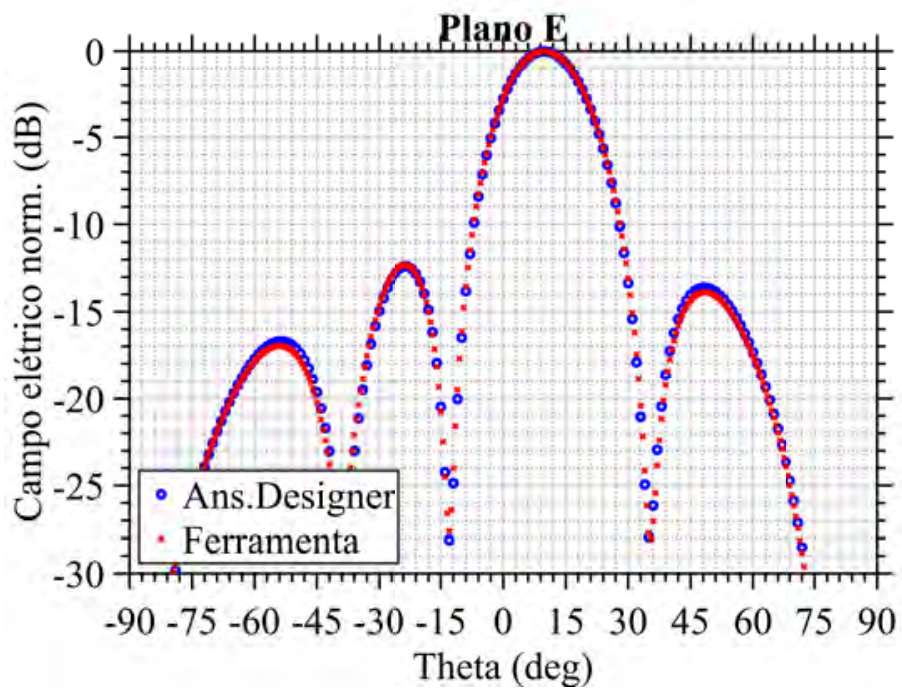

Figura 4.27: Campo calculado e simulado para uma rede $5 \times 5$ - onda plana com incidência oblíqua $\left(\theta_{i}=10^{\circ}\right)$. Plano E: $E_{\theta}\left(\theta, \phi=0^{\circ}\right)$.

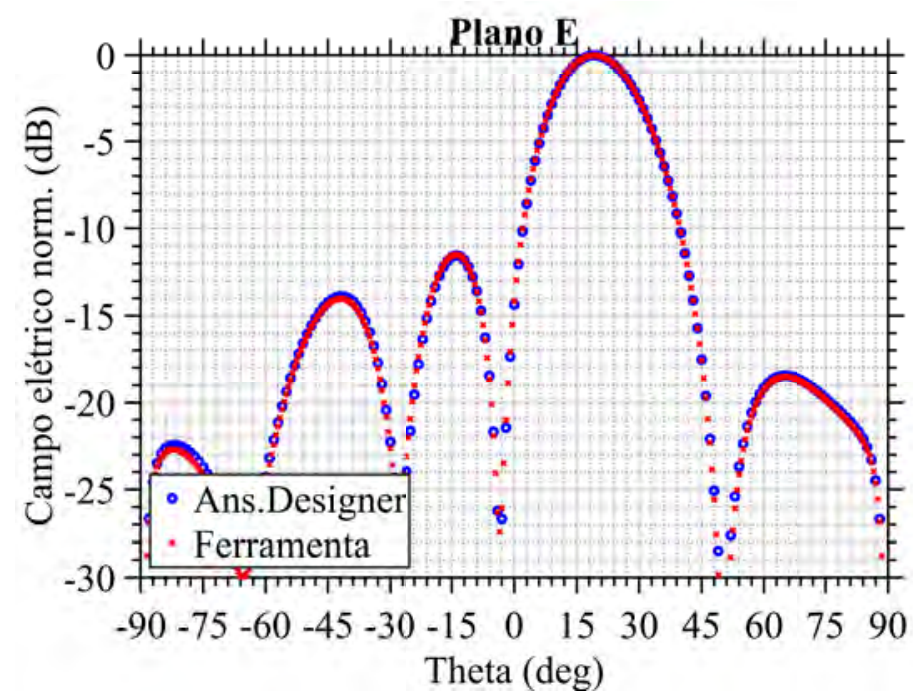

Figura 4.28: Campo calculado e simulado para uma rede $5 \times 5$ - onda plana com incidência oblíqua $\left(\theta_{i}=20^{\circ}\right)$. Plano E: $E_{\theta}\left(\theta, \phi=0^{\circ}\right)$. 
Capítulo 4. Análise de estruturas planares a partir do método dos momentos MoM

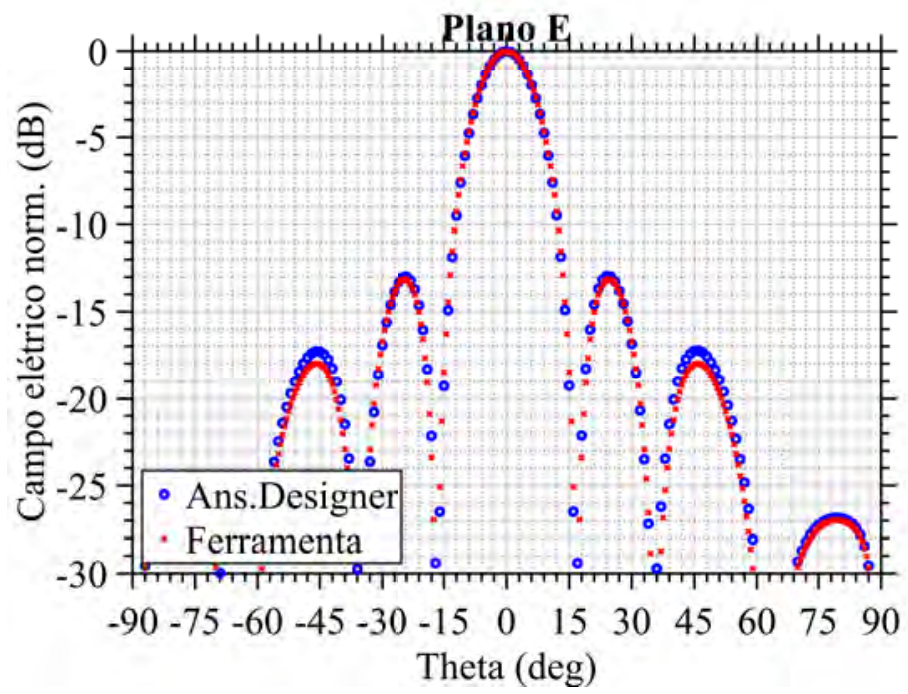

a)

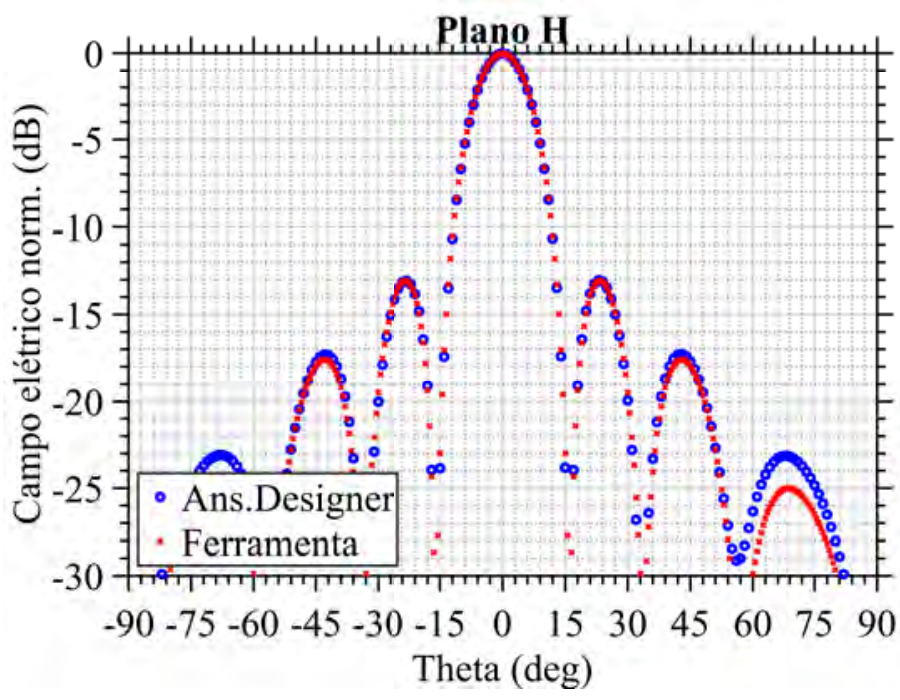

b)

Figura 4.29: Campo calculado e simulado para uma rede $7 \times 7$ - onda plana com incidência normal $\left(\theta_{i}=0^{\circ}\right)$. a) Plano E: $E_{\theta}\left(\theta, \phi=0^{\circ}\right)$; b) Plano $\mathrm{H}$ : $E_{\phi}\left(\theta, \phi=90^{\circ}\right)$. 


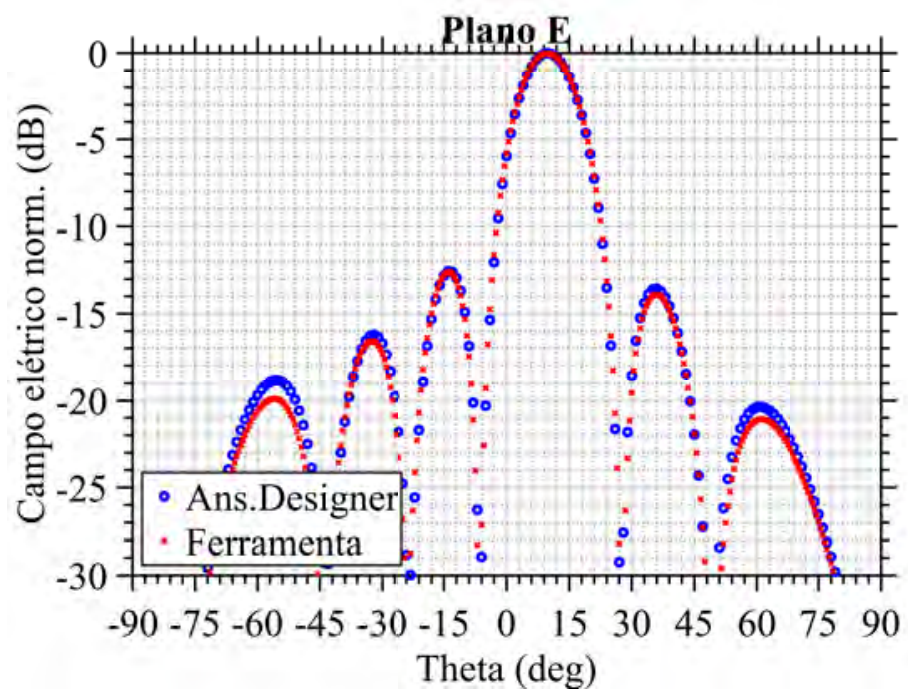

Figura 4.30: Campo calculado e simulado para uma rede $7 \times 7$ - onda plana com incidência oblíqua $\left(\theta_{i}=10^{\circ}\right)$. Plano E: $E_{\theta}\left(\theta, \phi=0^{\circ}\right)$.

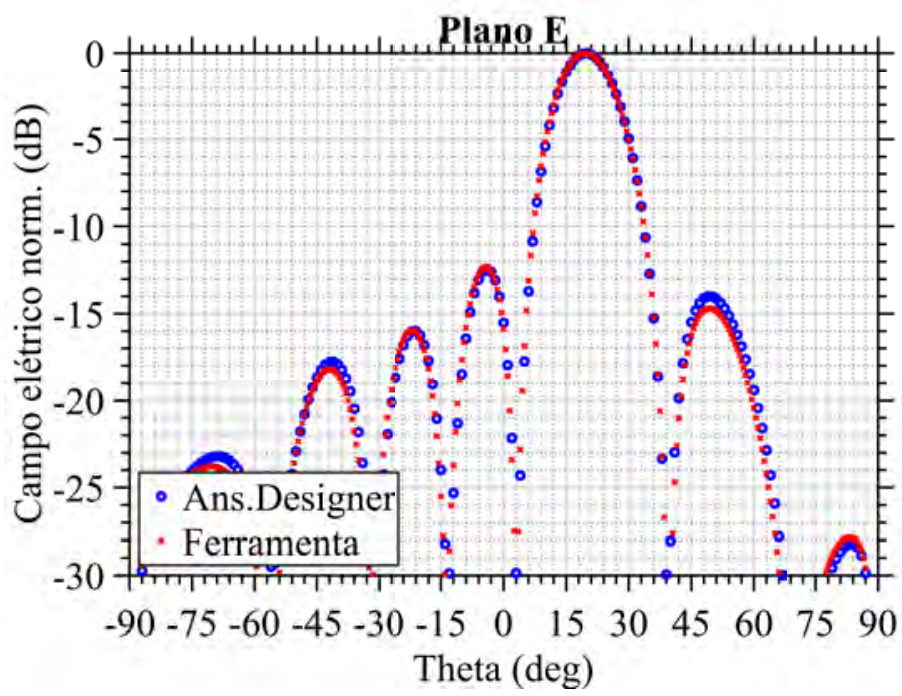

Figura 4.31: Campo calculado e simulado para uma rede $7 \times 7$ - onda plana com incidência oblíqua $\left(\theta_{i}=20^{\circ}\right)$. Plano E: $E_{\theta}\left(\theta, \phi=0^{\circ}\right)$.

Pode-se verificar que os diagramas de irradiação obtidos apresentam excelente concordância entre o Ansys Designer e a ferramenta baseada no método dos momentos e funções base com condição de borda apropriadamente segmentada.

Na Tabela 4.1 é apresentado o número de variáveis (pesos) a serem determinados a partir do MoM nas ferramentas comercial e proposta. Verificase que o Ansys Designer necessita um número muito superior para modelar 
Capítulo 4. Análise de estruturas planares a partir do método dos momentos MoM

a distribuição de corrente superficial sobre as metalizações. Adicionalmente, a ferramenta proposta necessita menor memória computacional.

Tabela 4.1: Número de variáveis desconhecidas a serem determinadas a partir do MoM.

\begin{tabular}{|c|c|c|}
\hline Estrutura & $\begin{array}{c}\text { Ferramenta } \\
\text { proposta }\end{array}$ & $\begin{array}{c}\text { Ansys } \\
\text { Designer }\end{array}$ \\
\hline Elemento & 2 & 332 \\
\hline RA $3 \times 3$ & 18 & 3.009 \\
\hline RA $5 \times 5$ & 50 & 5.852 \\
\hline RA $7 \times 7$ & 98 & 11.543 \\
\hline
\end{tabular}

\subsection{3}

\section{Campo elétrico espalhado e refletido por redes refletoras}

Com a verificação do campo espalhado através do Ansys Designer, incluem-se os efeitos da reflexão do campo incidente no substrato e plano de terra. As redes analisadas são as mesmas do espalhamento, porém utiliza-se também o Ansys HFSS na verificação. O plano de terra e o substrato possuem as seguintes dimensões físicas: $A=E_{x} \lambda_{0} / 2$ e $B=E_{y} \lambda_{0} / 2$, em que cada célula contém o tamanho de $C_{x}=\lambda_{0} / 2$ e $C_{y}=\lambda_{0} / 2$, conforme pode ser visualizado na Figura 4.32.

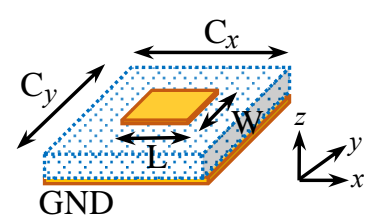

a)

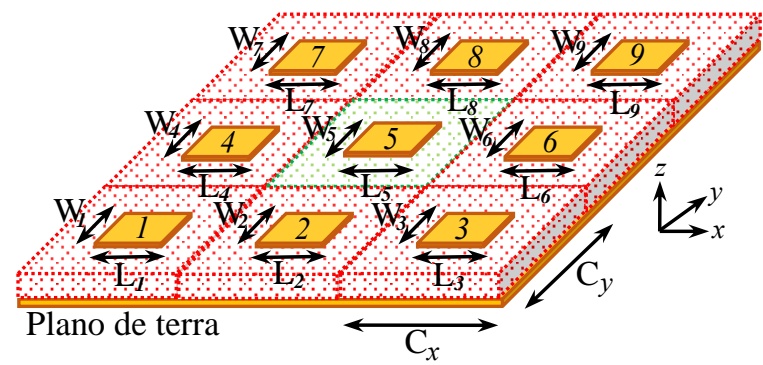

b)

Figura 4.32: Definição de células. a) Célula isolada; b) Célula central e demais células.

Inicialmente, é realizada uma análise paramétrica das dimensões $L$ e $W$ da metalização de uma célula isolada visando a obtenção da curva de fase, con- 
Capítulo 4. Análise de estruturas planares a partir do método dos momentos MoM

forme pode ser visualizado na Figura 4.33. Em comparação com a Figura 4.18, constata-se que a faixa de variação de fase foi ampliada em aproximadamente $180^{\circ}$ em decorrência da adição da componente do campo refletido no plano de terra. Adicionalmente, percebe-se que houve um deslocamento em frequência quando os resultados são comparados com o software HFSS. Este efeito é apresentado por diversos autores [106], e, conforme abordado, é característico da análise do campo elétrico espalhado através da função diádica de Green que considera um plano de terra infinito. Vale destacar que a curva de fase possui esse formato devido à correta relação entre o campo espalhado e refletido, em que o campo refletido é predominante em praticamente quase toda curva de fase, exceto em torno da dimensão de ressonância, o que produz essa variação rápida de fase do campo elétrico irradiado na direção especular.

Constata-se que o plano de terra/substrato infinito provoca um deslocamento da curva de fase em $70 \mathrm{MHz}$. Entretanto, a correção deste deslocamento indesejado pode ser facilmente realizado através do escalonamento da estrutura em frequência. Na Figura 4.33 pode ser observado que obtém-se excelente concordância entre o HFSS e a ferramenta após um escalonamento para 2,33 GHz.

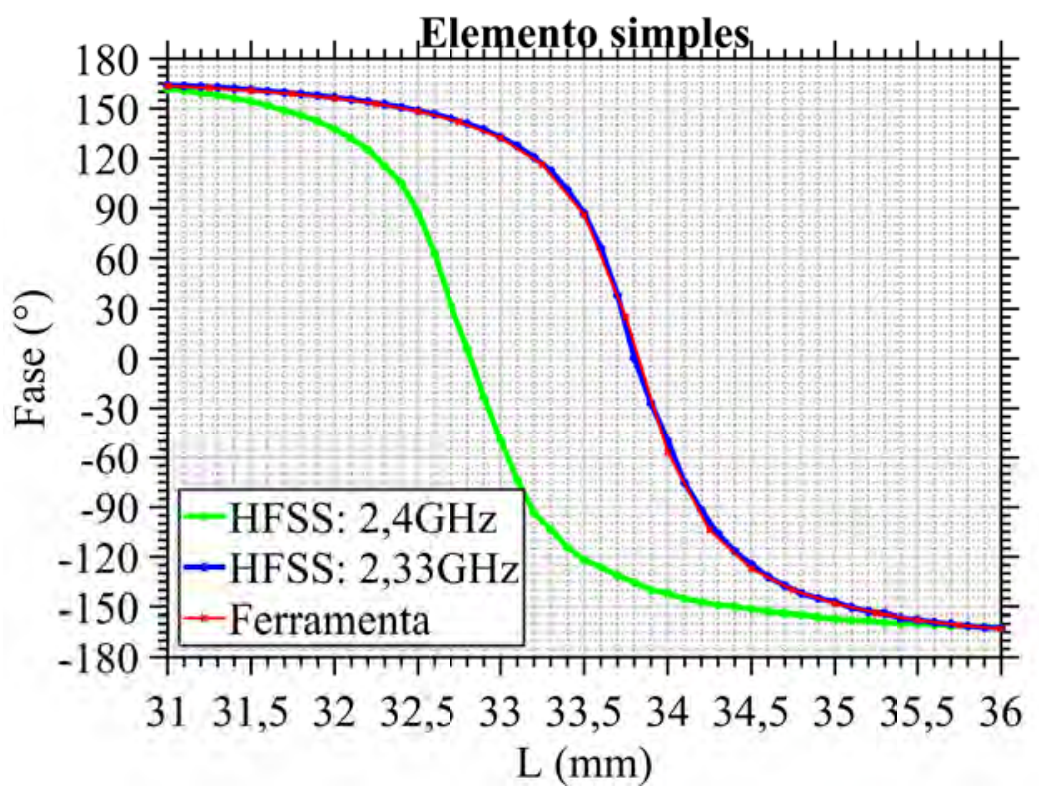

Figura 4.33: Escalonamento para correção de desvio em frequência.

Tomando como referência a Figura 4.32 b), avalia-se o comportamento da variação de fase de cada elemento na presença dos demais patches, de forma a incluir o efeito do acoplamento mútuo. A posição espacial de cada célula na rede impacta diretamente na intensidade da interação, resultando, assim, nas diferenças de fase para redes $3 \times 3,5 \times 5$ e $7 \times 7$ apresentadas nas Figuras 4.34 a 
Capítulo 4. Análise de estruturas planares a partir do método dos momentos MoM

4.36. Caso não existisse interferência eletromagnética, todas as curvas seriam idênticas à resposta da célula isolada.

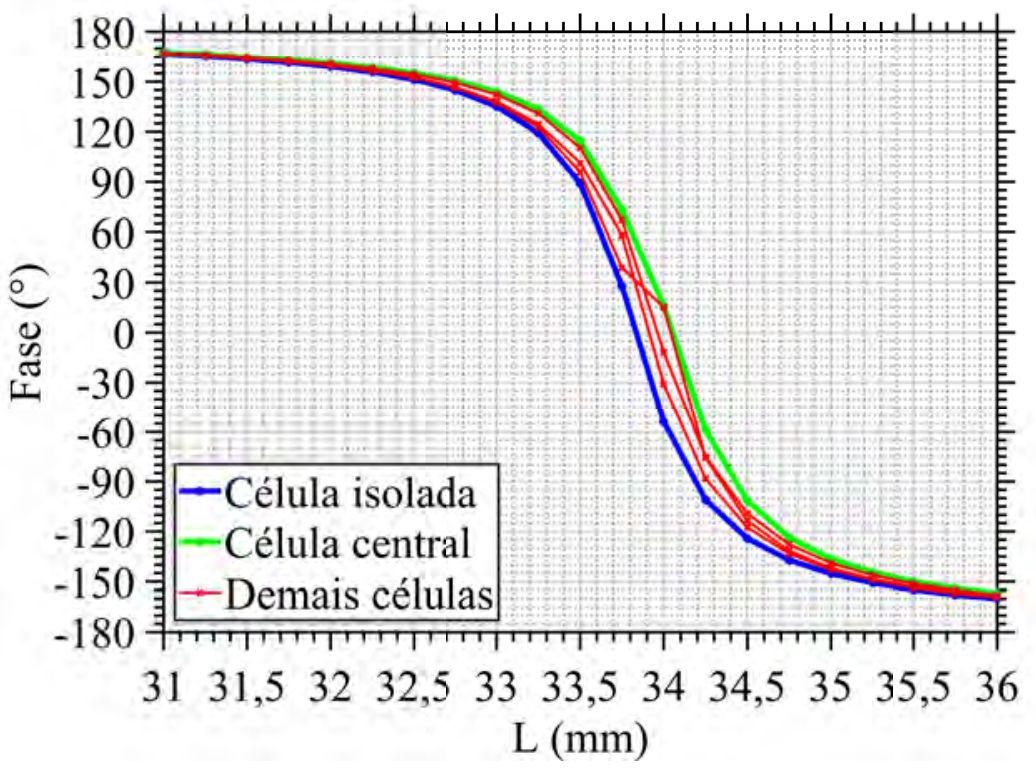

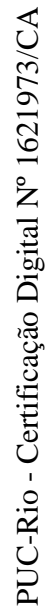

Figura 4.34: Comportamento da fase das células de uma RA $3 \times 3$ para incidência normal e observação broadside.

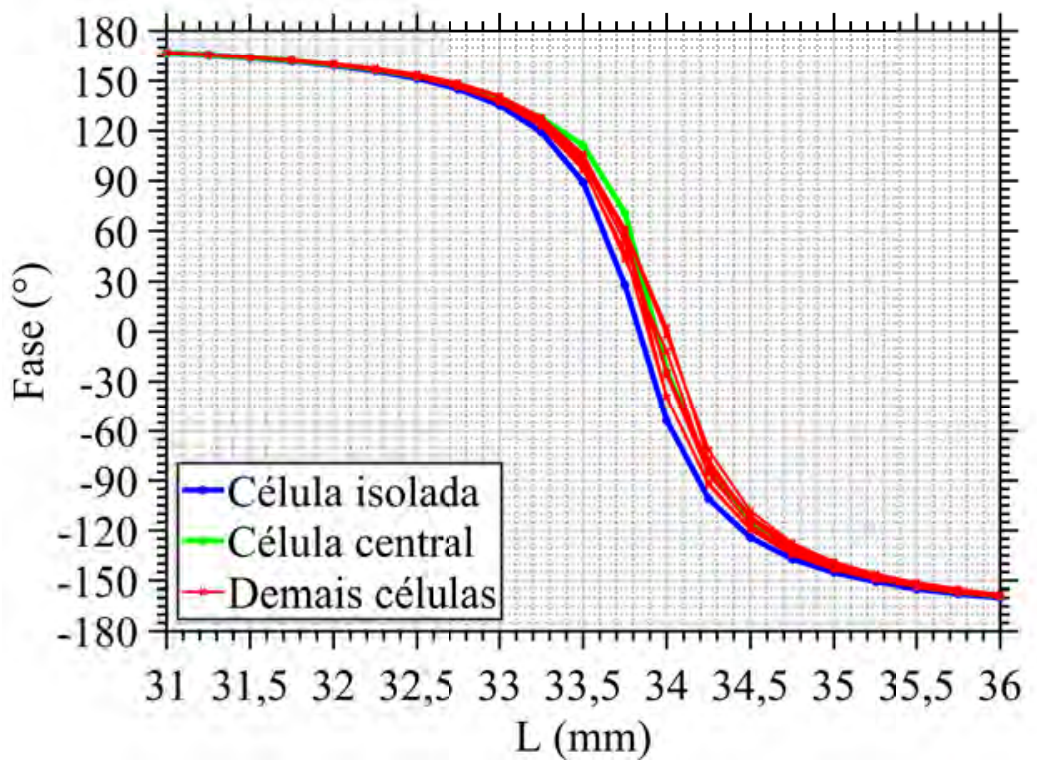

Figura 4.35: Comportamento da fase das células de uma RA $5 \times 5$ para incidência normal e observação broadside. 
Capítulo 4. Análise de estruturas planares a partir do método dos momentos MoM

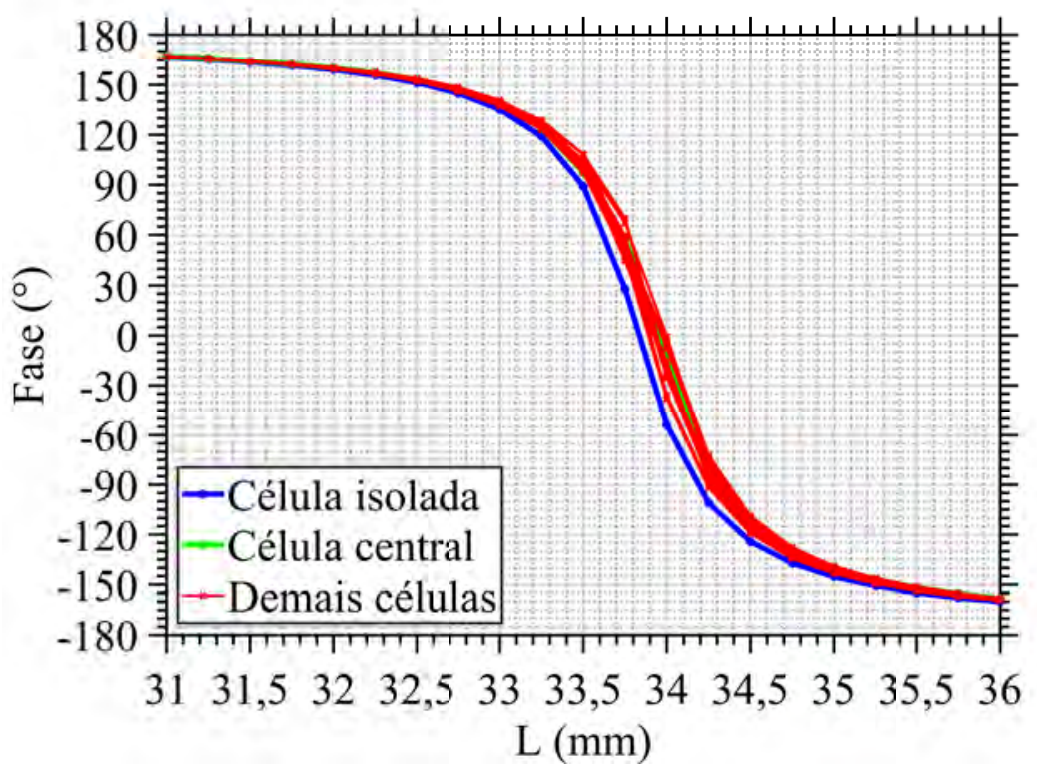

Figura 4.36: Comportamento da fase das células de uma RA $7 \times 7$ para incidência normal e observação broadside.

Muitos trabalhos encontrados na literatura consideram a abordagem de Floquet para o projeto de redes refletoras. Sendo assim, considera-se uma célula central na presença de uma rede infinita de elementos idênticos. Essa aproximação produz um erro de fase maior para estruturas de pequeno porte, podendo-se visualizar a diferença de fase das demais células em relação a célula central para as redes abordadas nas Figuras 4.37 a 4.39 .

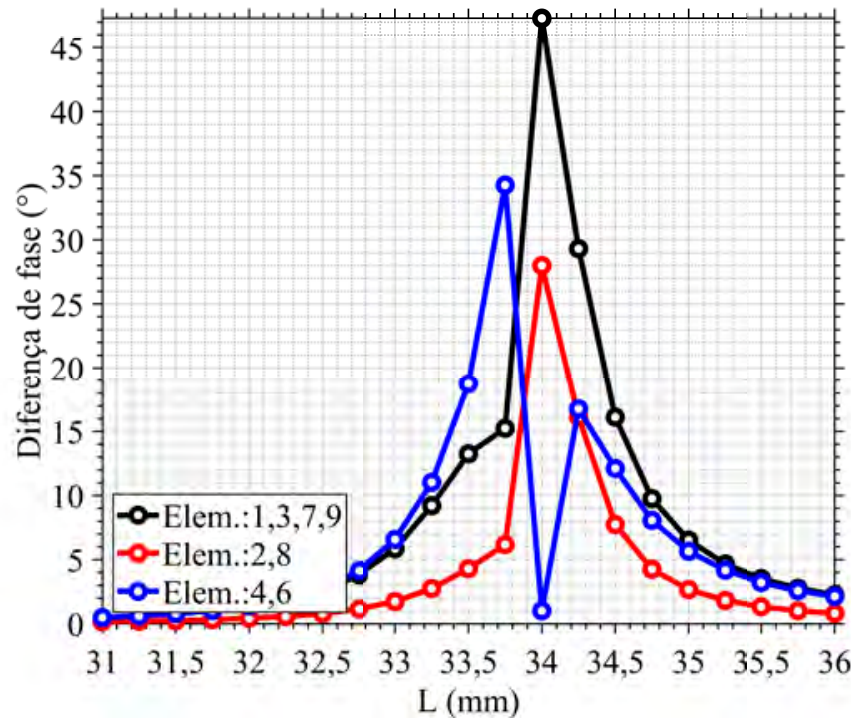

Figura 4.37: Diferença de fase das células de uma RA $3 \times 3$ em relação a célula central. 
Capítulo 4. Análise de estruturas planares a partir do método dos momentos MoM

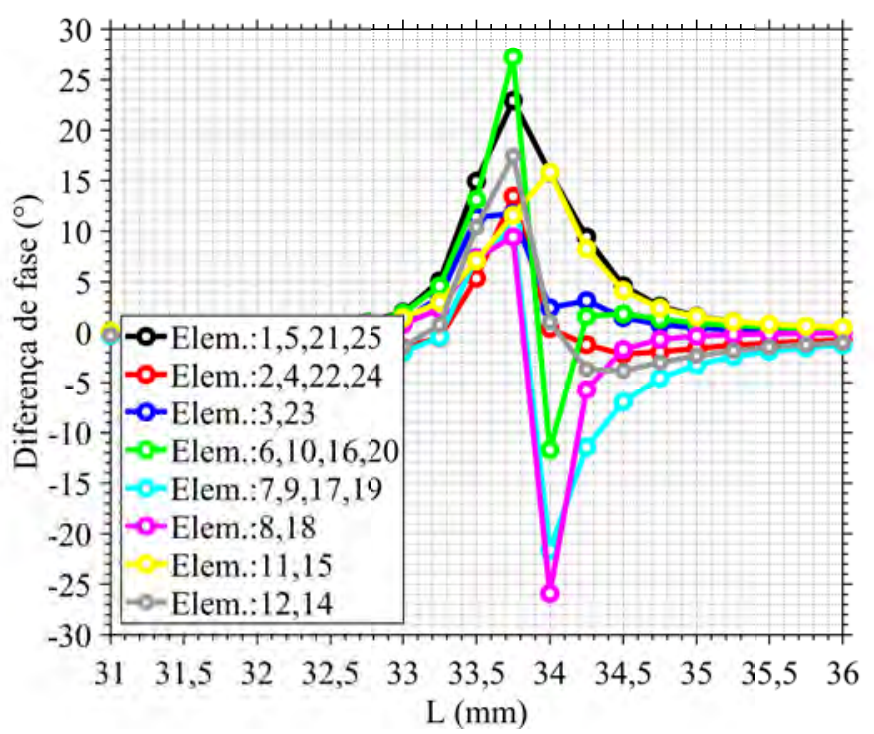

Figura 4.38: Diferença de fase das células de uma RA 5×5 em relação a célula central.

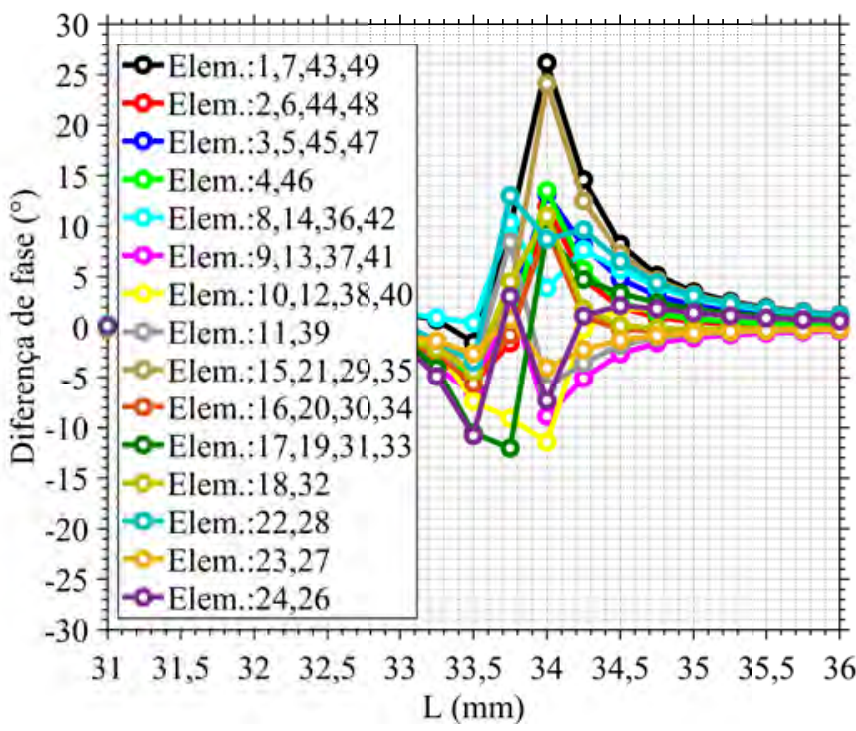

Figura 4.39: Diferença de fase das células de uma RA $7 \times 7$ em relação a célula central.

Percebe-se que a diferença de fase para a célula central diminui à medida que o tamanho da rede aumenta. Além disso, pode-se afirmar que maiores variações ocorrem para dimensões próximas da frequência de ressonância e, simultaneamente, para grande parte dos elementos da periferia (próximas da borda). 
Capítulo 4. Análise de estruturas planares a partir do método dos momentos MoM

Por este motivo, redes refletoras com grande número de elementos (RAs maiores que $15 \times 15$ ) aplicam a abordagem de Floquet, pois, além de minimizar o esforço computacional e tempo de simulação, apresentam resultados satisfatórios. Os efeitos do erro de fase introduzidos pelos elementos da borda sobre o campo total irradiado são minimizados pelos autores através da baixa iluminação nos extremos da rede, dada através de alimentadores específicos, os quais devem ser adequadamente projetados [107].

Na Figura 4.40 é feito o comparativo entre as curvas de fase de células centrais de redes $3 \times 3,5 \times 5$ e $7 \times 7$. Percebe-se que redes $5 \times 5$ e $7 \times 7$ apresentam curvas muito próximas, justificadas pelo motivo de que o acoplamento mútuo sobre o elemento central é pequeno para elementos mais afastados. Muitos autores descrevem que redes muito grandes são inviáveis de serem analisadas para a obtenção da curva de fase, porém mostra-se que não há necessidade de simular uma estrutura grande por completa e, sim, apenas parte dela. $\mathrm{Na}$ Figura 4.41 é apresentada a diferença de fase do elemento central de uma rede $7 \times 7$ (referência) em relação à célula isolada, e central de redes $3 \times 3$ e $5 \times 5$, sendo, no pior caso, $30^{\circ}, 14^{\circ}$ e $4^{\circ}$, respectivamente.

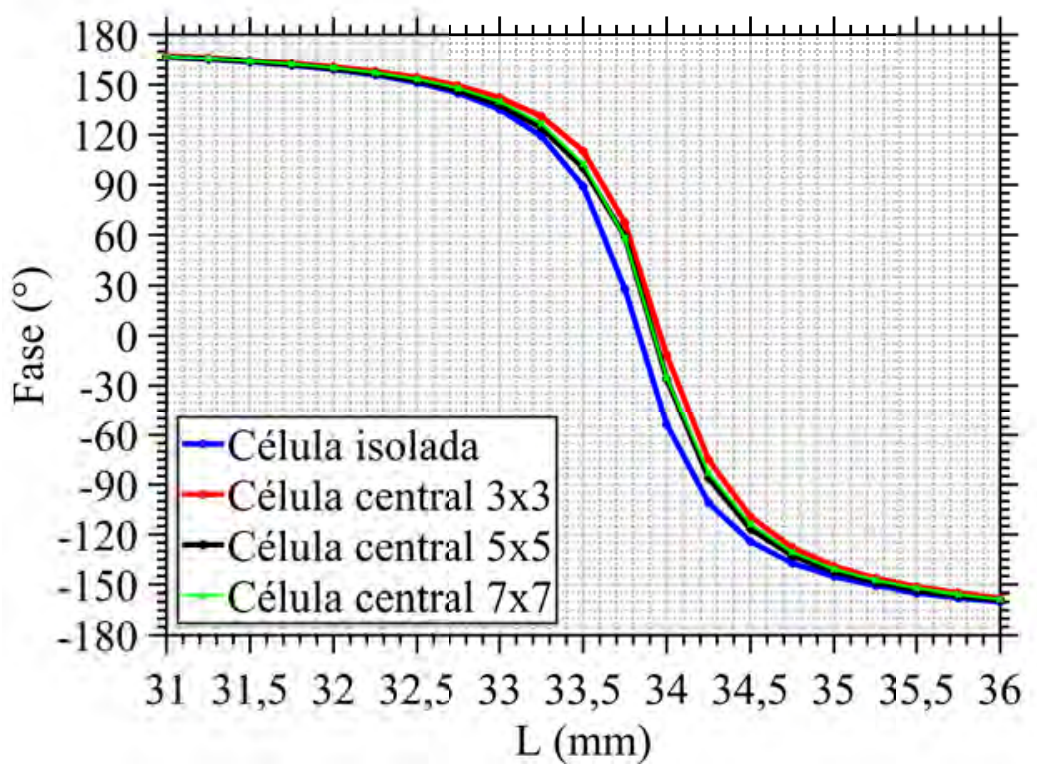

Figura 4.40: Comparativo das curvas de fase de células centrais de redes $3 \times 3$, $5 \times 5$ e $7 \times 7$. 


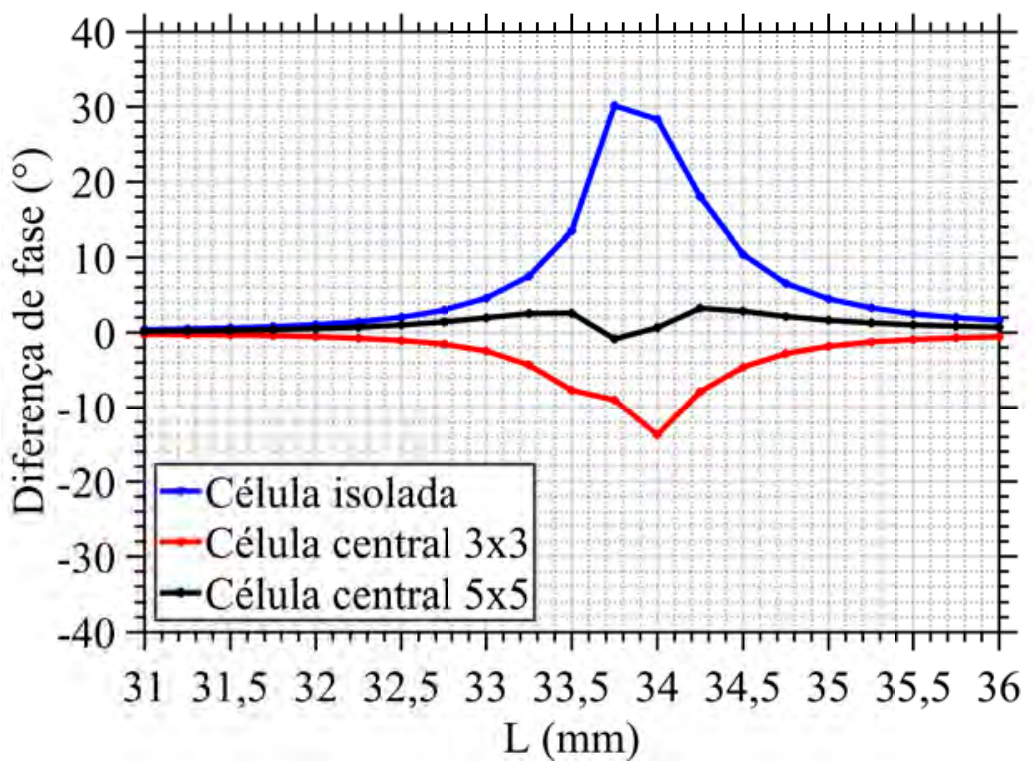

Figura 4.41: Diferença de fase da célula isolada e central de redes $3 \times 3$ e $5 \times 5$ para a célula central de uma RA $7 \times 7$.

Os próximos resultados apresentados tratam-se dos diagramas de irradiação para elemento simples, redes $3 \times 3,5 \times 5$ e $7 \times 7$, em que aplica-se a excitação por onda plana com incidências normal e oblíqua com inclinação de $10^{\circ}$ e $20^{\circ} \mathrm{e}$ utilizam-se patches de mesmo tamanho físico. Os resultados obtidos são comparados com o software Ansys HFSS na frequência de 2,33 GHz. Na Figura 4.42 tem-se o campo elétrico normalizado em dB para o elemento simples, nas Figuras 4.43 a 4.45 para redes $3 \times 3$, nas Figuras 4.46 a 4.48 para redes $5 \times 5$ e nas Figuras 4.49 a 4.51 para redes $7 \times 7$.

Em relação aos diagramas no plano E, constata-se boa concordância entre os resultados, apresentando pequena diferença após o último nulo. Com relação ao plano $\mathrm{H}$, verifica-se que a intensidade dos lóbulos laterais apresentam pequena diferença de amplitude, que é causada pela difração de borda que está incluso apenas nos dados exportados a partir do HFSS. A inclusão dos efeitos de difração de borda nos resultados da técnica proposta é apresentada na seção seguinte. 
Capítulo 4. Análise de estruturas planares a partir do método dos momentos MoM

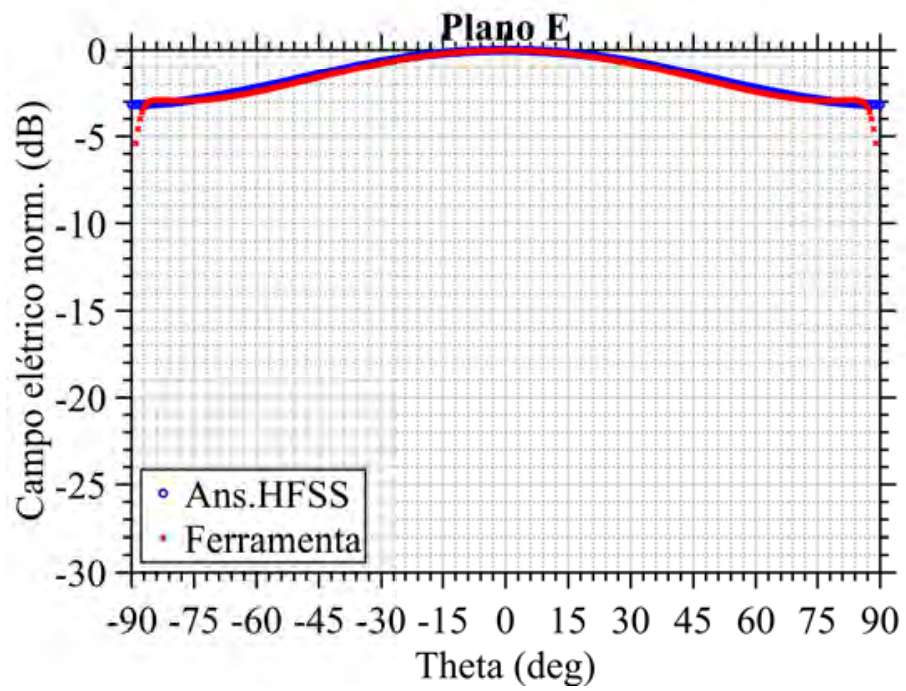

a)

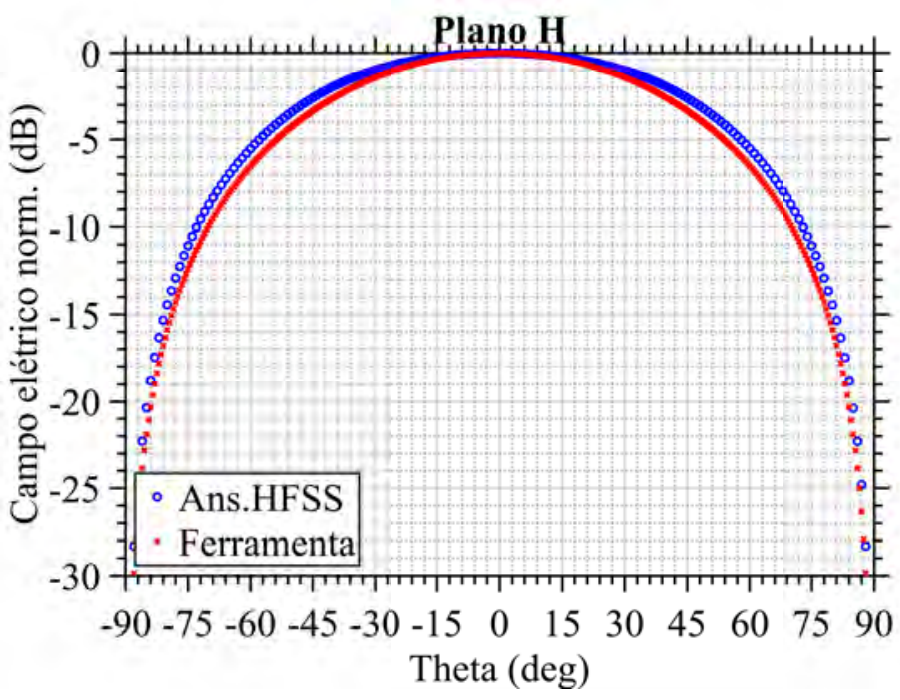

b)

Figura 4.42: Campo calculado e simulado para um elemento simples - onda plana com incidência normal. a) Plano E: $E_{\theta}\left(\theta, \phi=0^{\circ}\right)$; b) Plano $\mathrm{H}: E_{\phi}(\theta, \phi=$ $\left.90^{\circ}\right)$. 
Capítulo 4. Análise de estruturas planares a partir do método dos momentos MoM

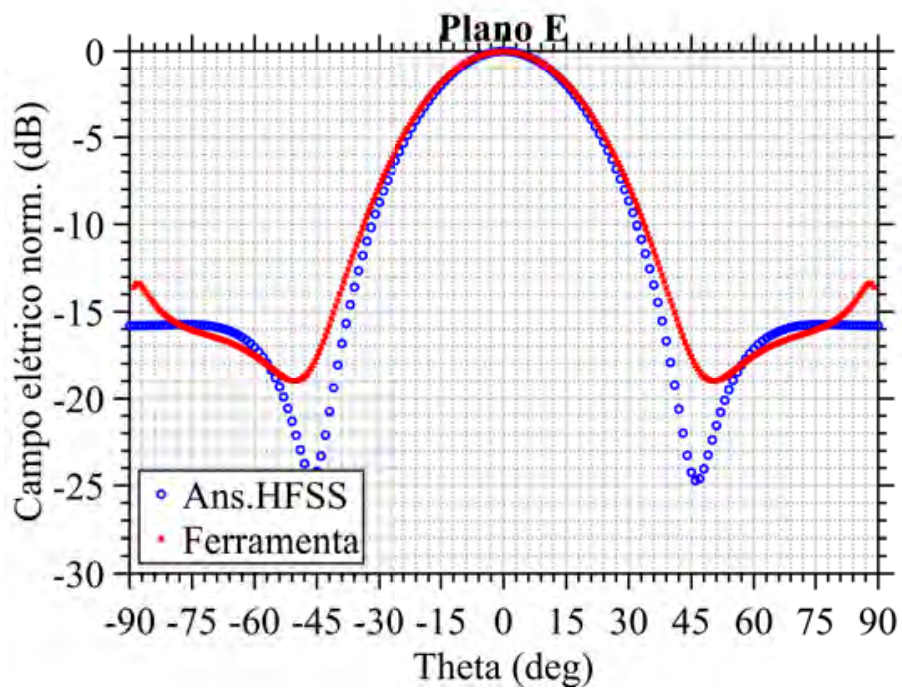

a)

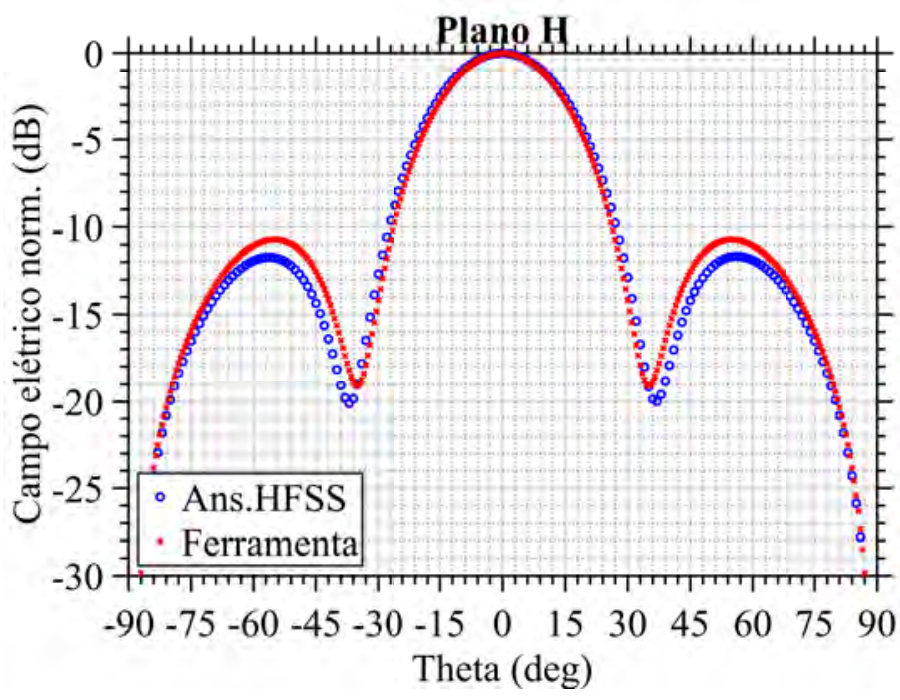

b)

Figura 4.43: Campo calculado e simulado para uma rede $3 \times 3$ - onda plana com incidência normal. a) Plano E: $E_{\theta}\left(\theta, \phi=0^{\circ}\right)$; b) Plano $\mathrm{H}: E_{\phi}\left(\theta, \phi=90^{\circ}\right)$. 
Capítulo 4. Análise de estruturas planares a partir do método dos momentos MoM

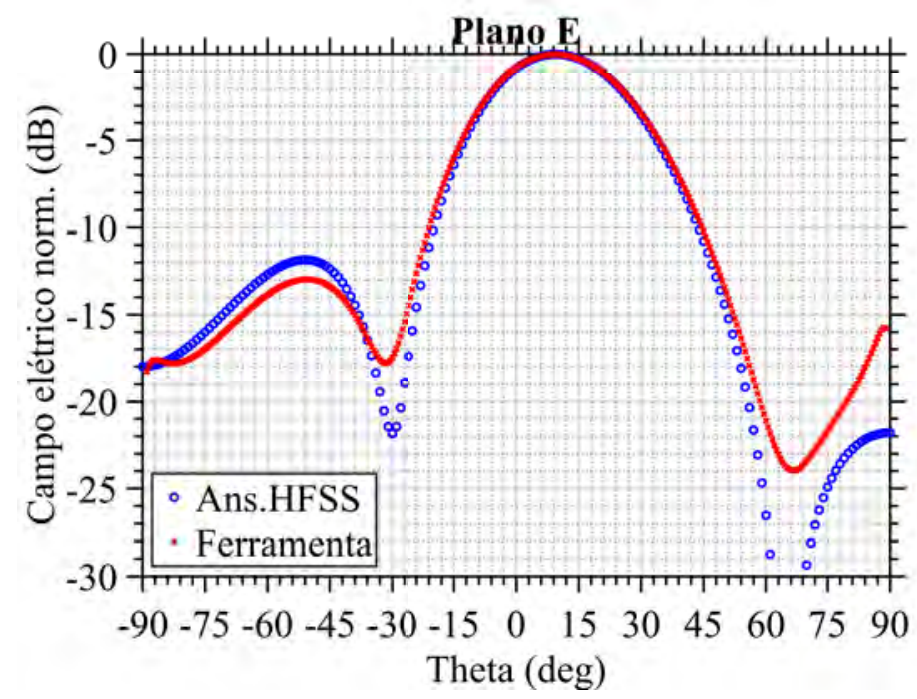

Figura 4.44: Campo calculado e simulado para uma rede $3 \times 3$ - onda plana com incidência oblíqua $\left(\theta_{i}=10^{\circ}\right)$. Plano E: $E_{\theta}\left(\theta, \phi=0^{\circ}\right)$.

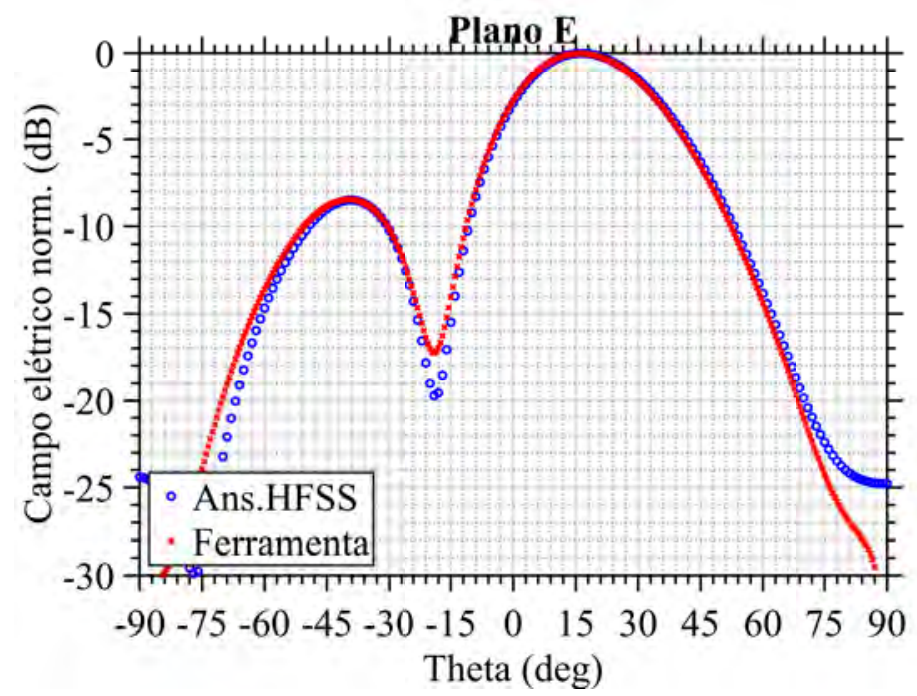

Figura 4.45: Campo calculado e simulado para uma rede $3 \times 3$ - onda plana com incidência oblíqua $\left(\theta_{i}=20^{\circ}\right)$. Plano E: $E_{\theta}\left(\theta, \phi=0^{\circ}\right)$. 
Capítulo 4. Análise de estruturas planares a partir do método dos momentos MoM

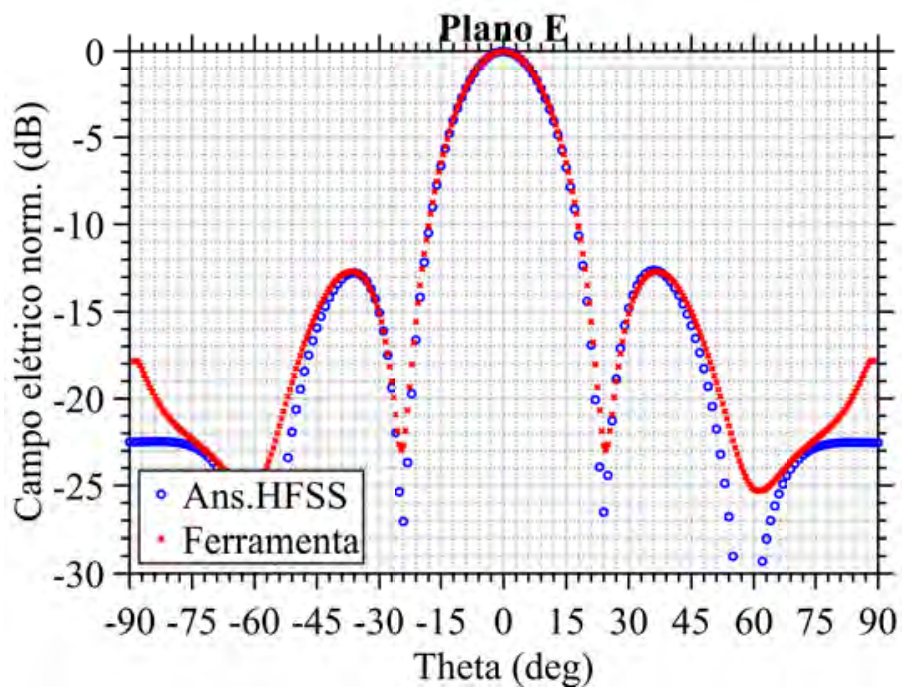

a)

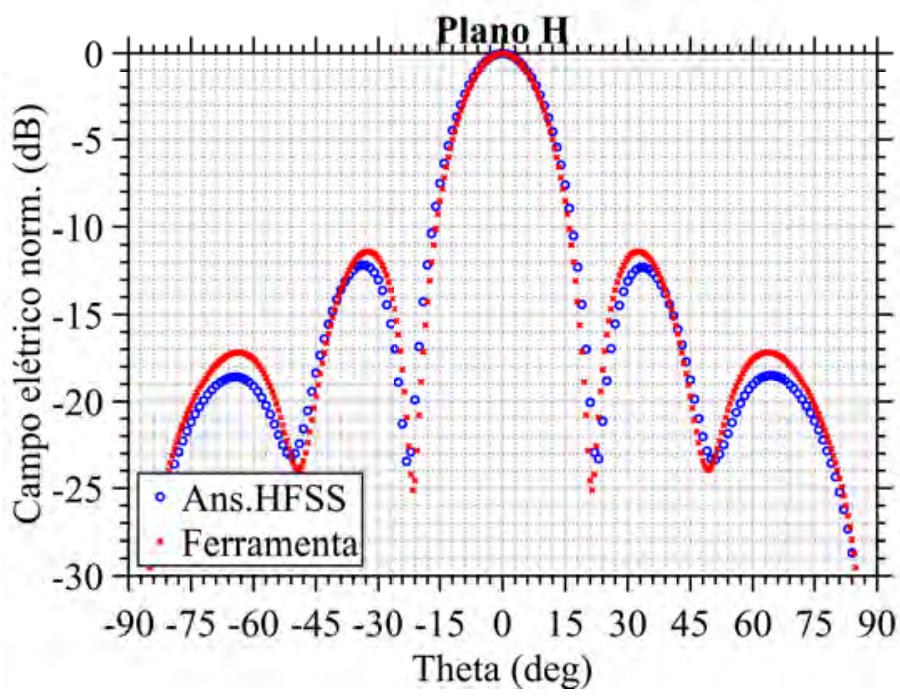

b)

Figura 4.46: Campo calculado e simulado para uma rede $5 \times 5$ - onda plana com incidência normal. a) Plano E: $E_{\theta}\left(\theta, \phi=0^{\circ}\right)$; b) Plano $\mathrm{H}: E_{\phi}\left(\theta, \phi=90^{\circ}\right)$. 
Capítulo 4. Análise de estruturas planares a partir do método dos momentos MoM

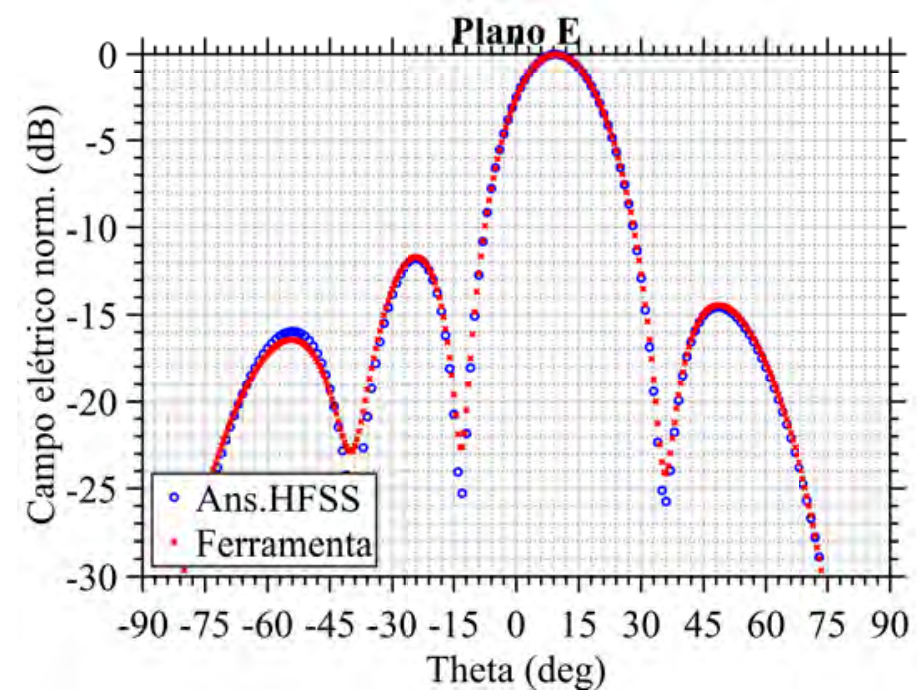

Figura 4.47: Campo calculado e simulado para uma rede $5 \times 5$ - onda plana com incidência oblíqua $\left(\theta_{i}=10^{\circ}\right)$. Plano E: $E_{\theta}\left(\theta, \phi=0^{\circ}\right)$.

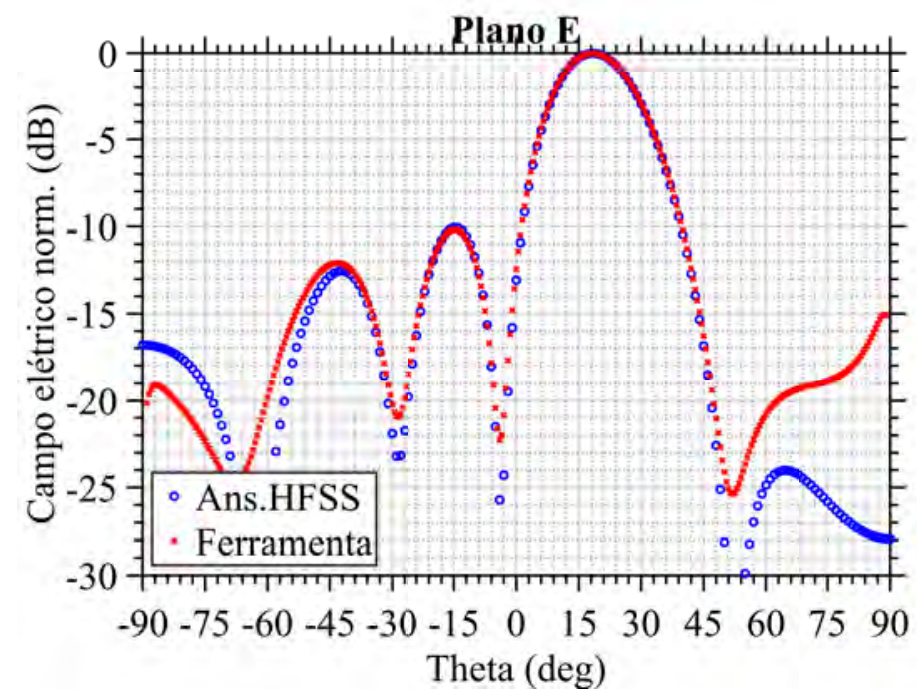

Figura 4.48: Campo calculado e simulado para uma rede $5 \times 5$ - onda plana com incidência oblíqua $\left(\theta_{i}=20^{\circ}\right)$. Plano E: $E_{\theta}\left(\theta, \phi=0^{\circ}\right)$. 
Capítulo 4. Análise de estruturas planares a partir do método dos momentos MoM

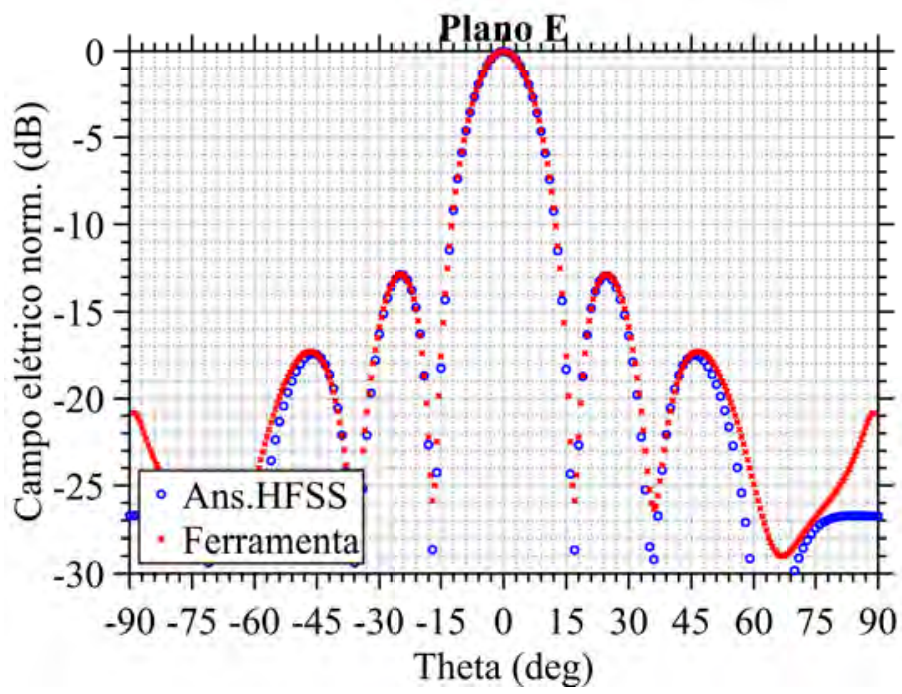

a)

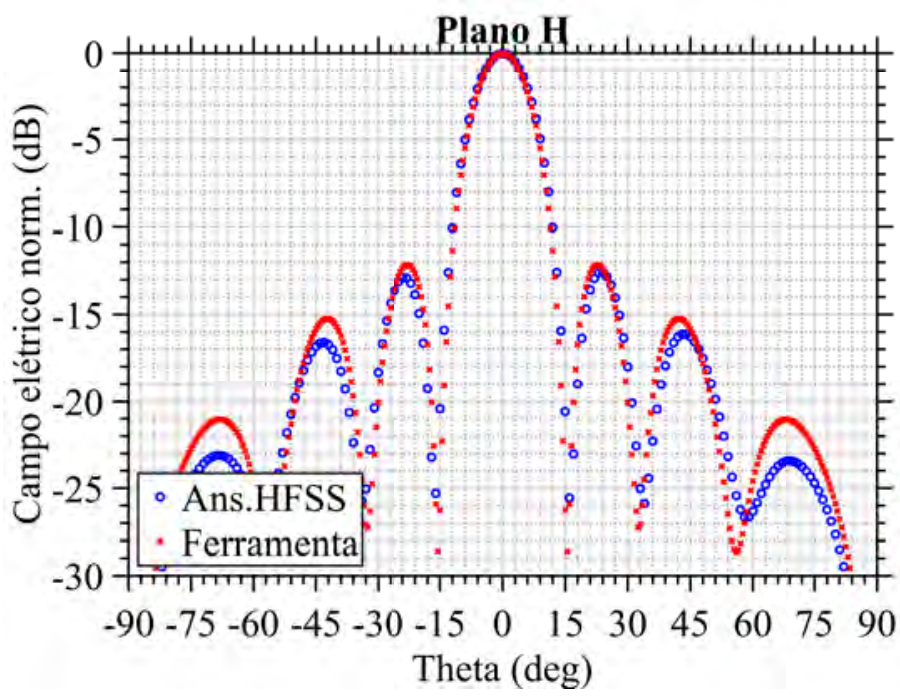

b)

Figura 4.49: Campo calculado e simulado para uma rede $7 \times 7$ - onda plana com incidência normal. a) Plano E: $E_{\theta}\left(\theta, \phi=0^{\circ}\right)$; b) Plano $\mathrm{H}: E_{\phi}\left(\theta, \phi=90^{\circ}\right)$. 
Capítulo 4. Análise de estruturas planares a partir do método dos momentos MoM

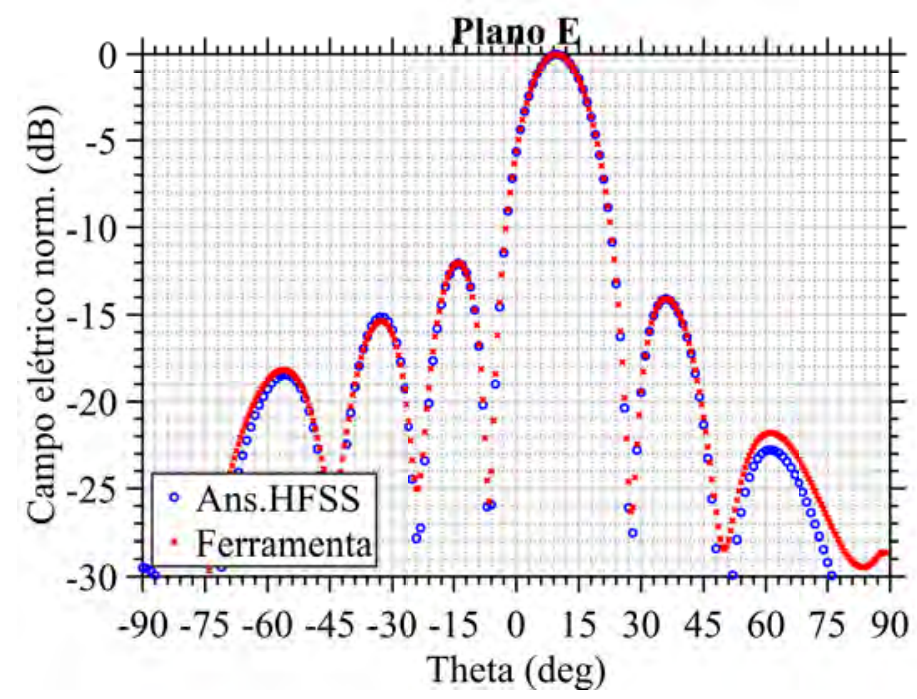

Figura 4.50: Campo calculado e simulado para uma rede $7 \times 7$ - onda plana com incidência oblíqua $\left(\theta_{i}=10^{\circ}\right)$. Plano E: $E_{\theta}\left(\theta, \phi=0^{\circ}\right)$.

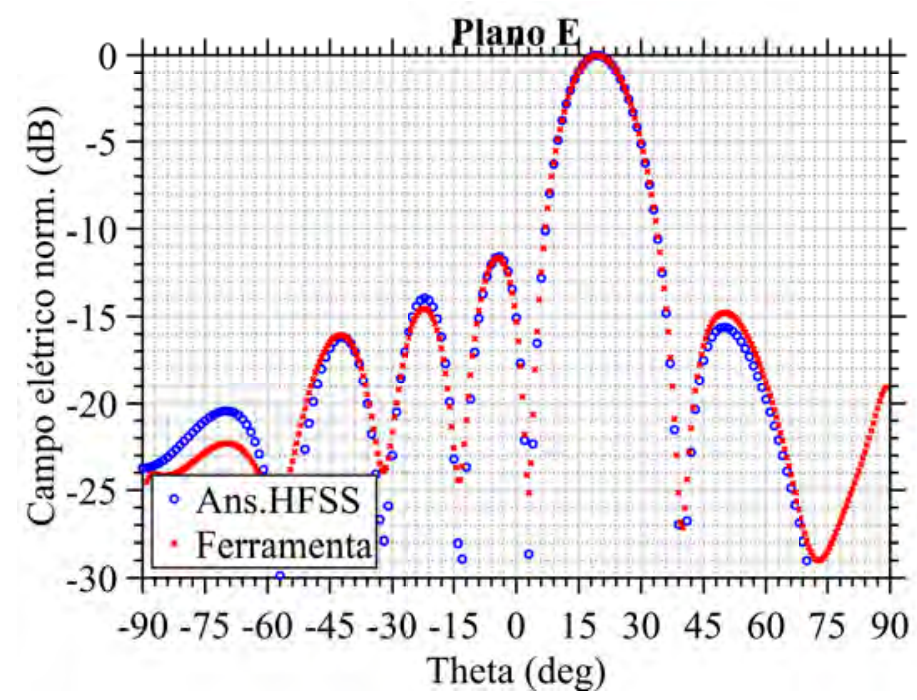

Figura 4.51: Campo calculado e simulado para uma rede $7 \times 7$ - onda plana com incidência oblíqua $\left(\theta_{i}=20^{\circ}\right)$. Plano E: $E_{\theta}\left(\theta, \phi=0^{\circ}\right)$.

\subsection{4}

\section{Campo elétrico espalhado, refletido e difratado por redes refletoras}

Nesta seção é adicionado vetorialmente o campo difratado pelo plano de terra da rede refletora no cálculo do campo elétrico total re-irradiado. Para fins de simplificação na determinação do campo difratado nas bordas da estrutura, a cobertura dielétrica é desprezada devido à fina espessura em termos do comprimento de onda. Adicionalmente, considera-se apenas a 
difração de primeira ordem. Em análises de RAs a partir da aplicação de Floquet, não é possível avaliar este efeito sobre a curva de fase de cada elemento da rede, sendo assim, o campo difratado apenas pode ser atribuído diretamente no campo total irradiado.

Neste trabalho o efeito do campo difratado na borda do plano de terra é avaliado e adicionado ao campo elétrico produzido por células pertencentes à borda da rede refletora, como, por exemplo, a difração devido à borda \#1 é inclusa no campo individual dos elementos 1, 4 e 7, da borda \#2 nos elementos 1, 2 e 3, da borda \#3 nos elementos 3, 6 e 9 e da borda \#4 nos elementos 7, 8 e 9 da Figura 4.52.

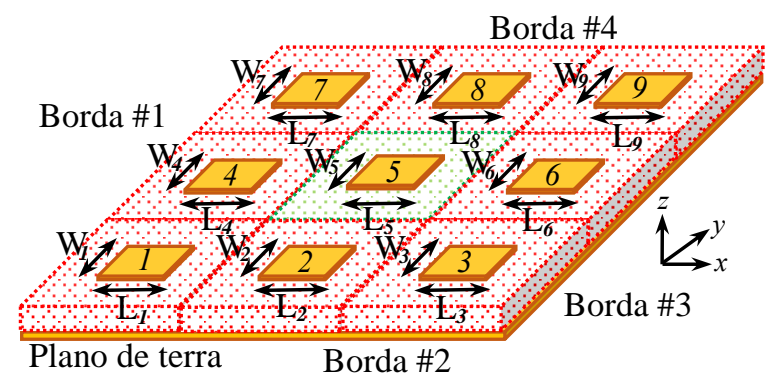

Figura 4.52: Definição das bordas de células pertencentes a periferia de redes refletoras.

A partir disso, a análise paramétrica das dimensões dos patches é realizada e as curvas de fase dos elementos são obtidas para uma iluminação normal e observação broadside. As respostas são ilustradas nas Figuras 4.53, 4.54 e 4.55 . Vale destacar que a curva de fase para a célula central é a mesma do calculado anteriormente a partir da soma dos campos espalhado e refletido, visto que a célula central não possui bordas. 
Capítulo 4. Análise de estruturas planares a partir do método dos momentos MoM

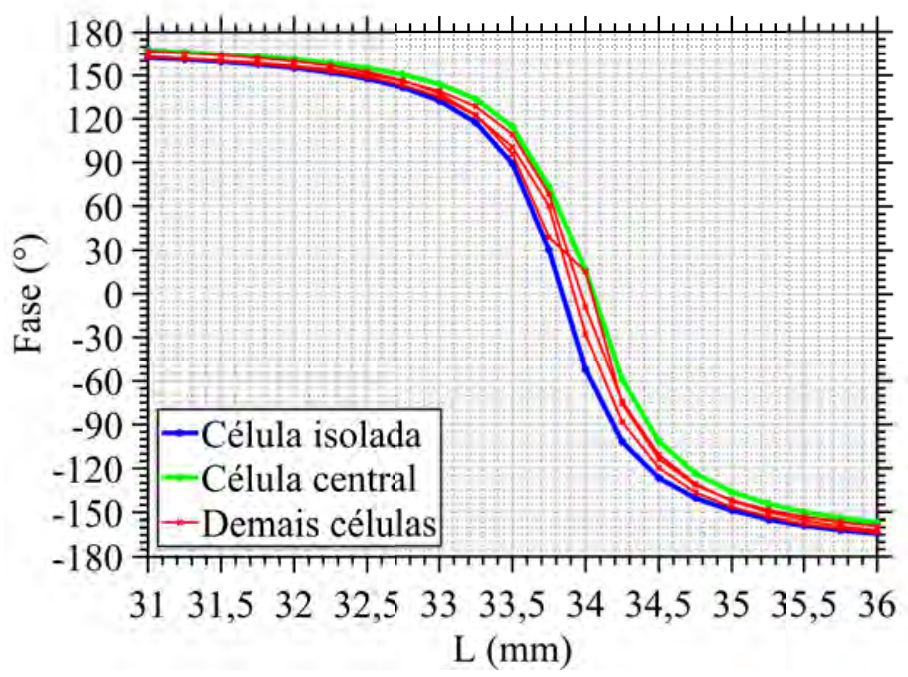

Figura 4.53: Comportamento da fase das células de uma RA $3 \times 3$ com a inclusão de efeitos difrativos.

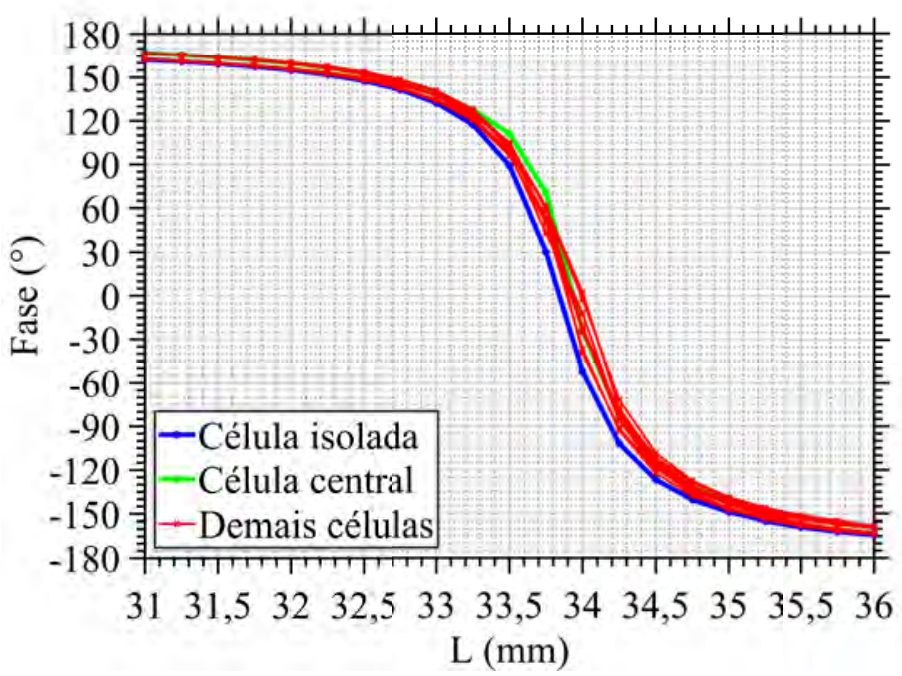

Figura 4.54: Comportamento da fase das células de uma RA $5 \times 5$ com a inclusão de efeitos difrativos. 


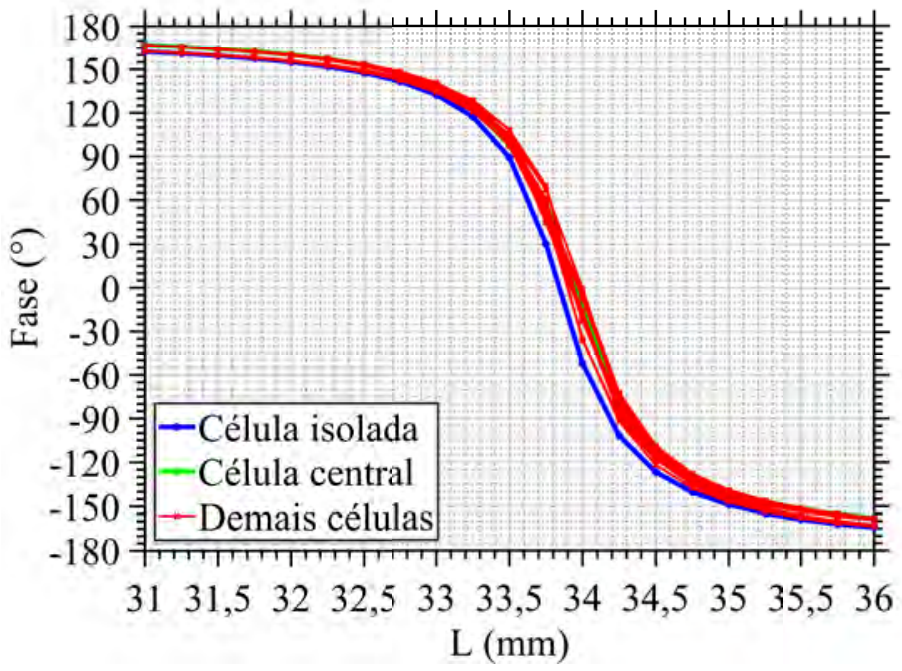

Figura 4.55: Comportamento da fase das células de uma RA $7 \times 7$ com a inclusão de efeitos difrativos.

O próximo passo é analisar a diferença de fase entre as curvas com e sem difração. Conforme ilustrado nas Figuras 4.56 a 4.58, percebe-se que a diferença de fase em relação a célula central em todos os casos é praticamente desprezível. Como justificativa, pode-se afirmar que os efeitos de difração são mais intensos no plano H. A curva é obtida a partir da fase do campo irradiado na direção especular do plano E, e, para este caso, a difração é pouco significativa.

Outra observação é de que apenas algumas células apresentaram diferenças, e são aquelas pertencentes às bordas específicas. Neste caso, o plano E é apenas afetado pela difração ocorrida nas bordas \#2 e \#4, e, assim, tomando como base a Figura 4.52, as células 4 e 6 não possuem variações em seus respectivos campos totais irradiados, e, consequentemente, as curvas de fase permanecem inalteradas, fato este apresentado na Figura 4.56. A respectiva interpretação pode ser facilmente aplicada para as demais redes analisadas. Todavia, as células 4 e 6 contribuem juntamente com as células 1, 3, 7 e 9 (pertencentes às bordas \#1 e \#3) para o diagrama no plano $\mathrm{H}$. 
Capítulo 4. Análise de estruturas planares a partir do método dos momentos MoM

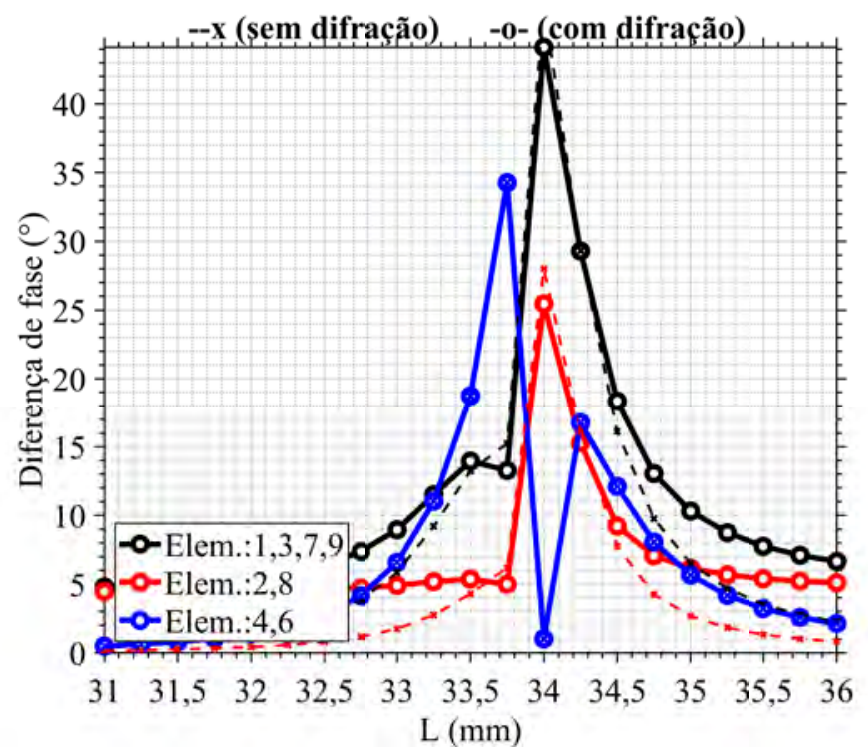

Figura 4.56: Diferença de fase das células de uma RA $3 \times 3$ para a célula central.

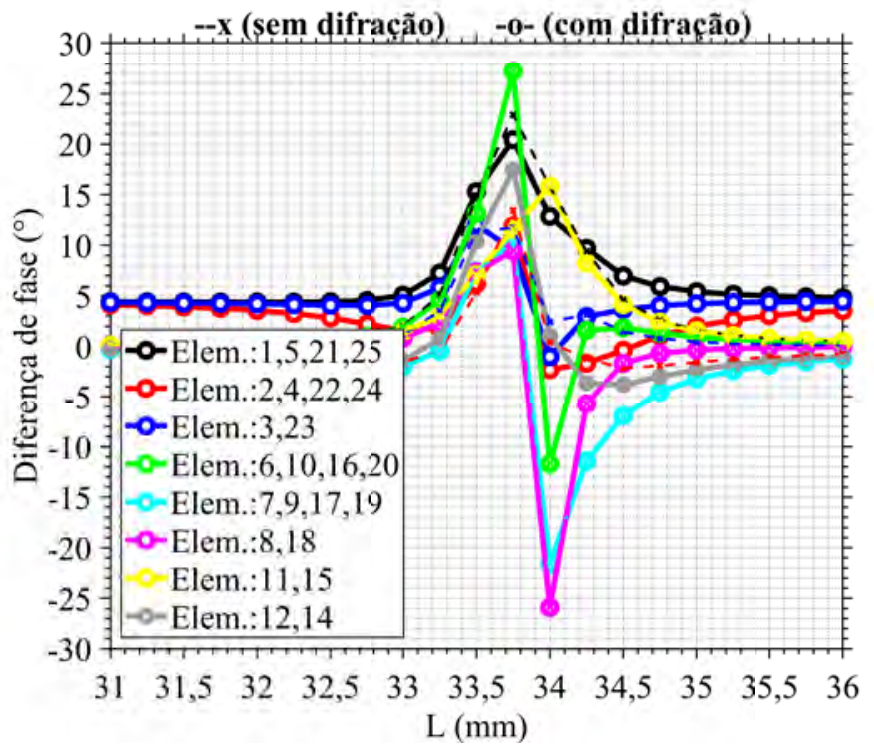

Figura 4.57: Diferença de fase das células de uma RA $5 \times 5$ para a célula central. 


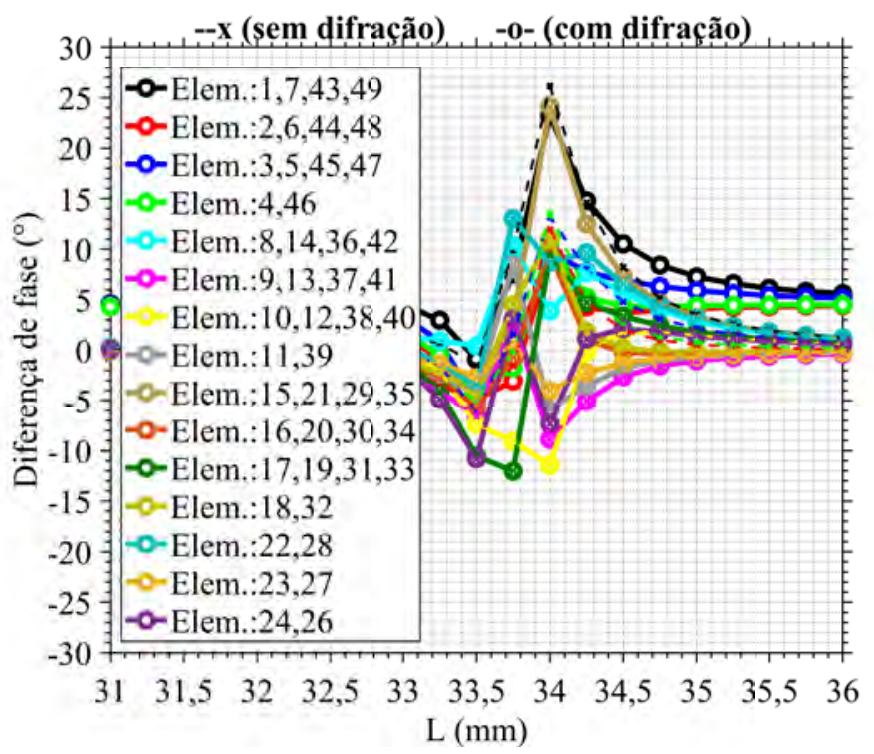

Figura 4.58: Diferença de fase das células de uma RA $7 \times 7$ para a célula central.

Os próximos resultados apresentados tratam-se dos diagramas de irradiação com a adiação do campo elétrico difratado aos demais campos computados anteriormente. Nas Figuras 4.59 a 4.61 tem-se o campo elétrico normalizado para redes $3 \times 3$, nas Figuras 4.62 a 4.64 para redes $5 \times 5$ e nas Figuras 4.65 a 4.67 para redes $7 \times 7$.

Os diagramas de irradiação obtidos apresentam boa concordância com o software comercial. Para o plano H, verifica-se que o campo elétrico total irradiado aproximou melhor o lóbulo mais afastado do principal, região essa afetada pela difração de borda. Já para o plano E, percebe-se diferença entre os diagramas para regiões a partir do último nulo, sendo assim, menos perceptível quando as dimensões da rede aumentam.

Após uma análise minuciosa, verificam-se variações de amplitude e fase do campo espalhado nas direções em que as discrepâncias ocorrem, erro este introduzido devido à consideração de um plano de terra e substrato infinitos. Uma possível causa deste erro é que ondas de superfície, presentes na formulação da função diádica de Green devido às dimensões da estrutura serem infinitas, estejam interferindo no campo elétrico irradiado nestas direções específicas. Vale destacar que a estrutura é finita no HFSS, sendo assim, ondas de superfície não estão presentes neste caso, porém, o campo irradiado pela lateral da rede refletora é determinado pelo software eletromagnético. A possibilidade de aumentar as dimensões do plano de terra e substrato no HFSS poderia ser aplicada para buscar um diagrama no software comercial que mais se aproxime do resultado da ferramenta proposta, todavia, esse 
Capítulo 4. Análise de estruturas planares a partir do método dos momentos MoM

estudo poderia ser utilizado apenas em antenas de microfita cuja alimentação é realizada, por exemplo, por prova coaxial. No presente caso, a iluminação das metalizações ocorre através de uma onda plana. Desta forma, se as dimensões fossem aumentadas para aproximar o campo espalhado, o campo refletido seria modificado devido ao tamanho da abertura. Sendo assim, devido às características do software, este efeito não pôde ser validado.

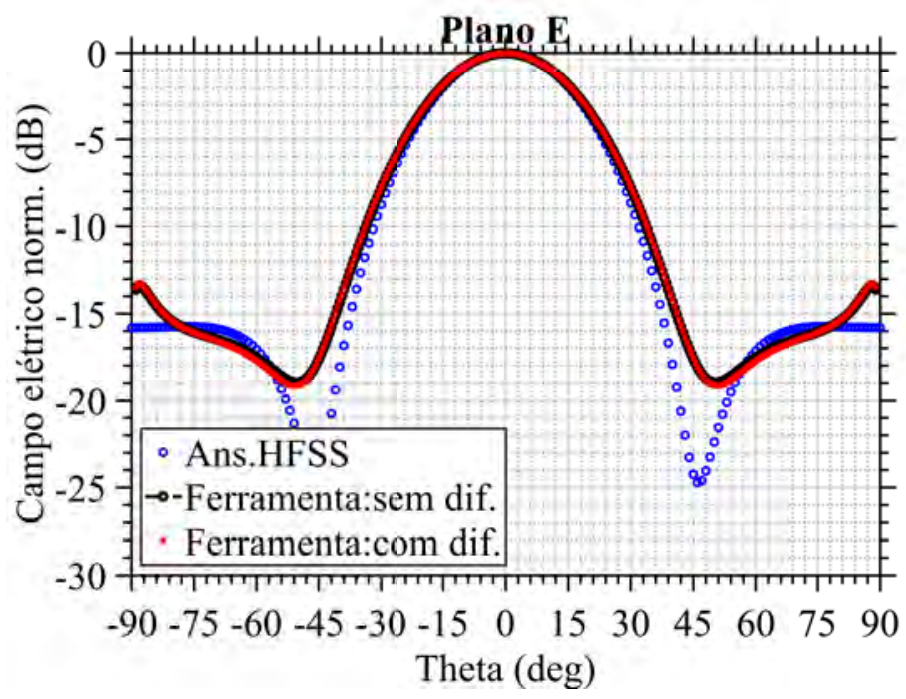

a)

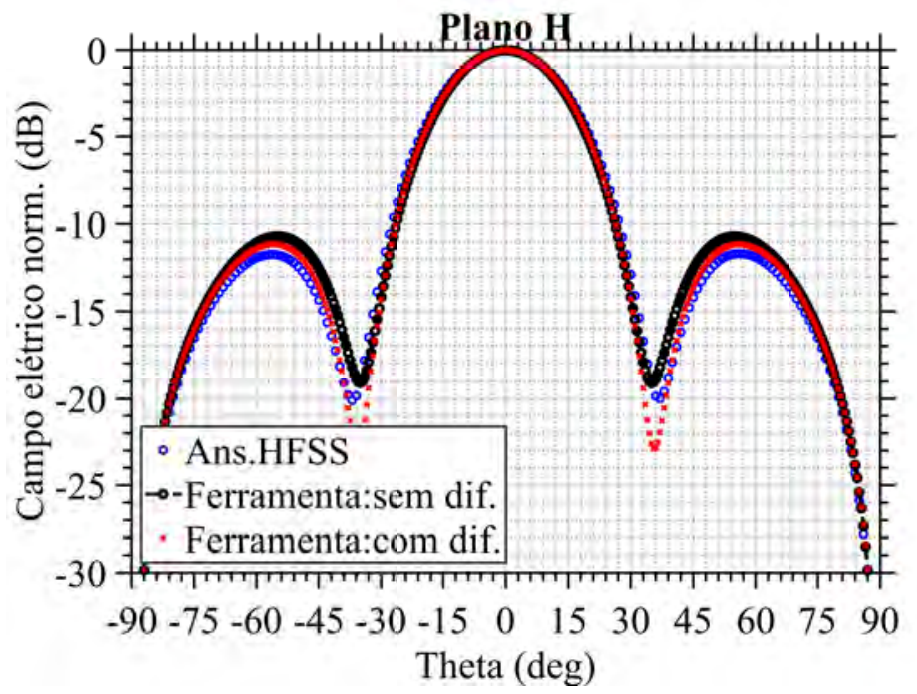

b)

Figura 4.59: Campo calculado e simulado para uma rede $3 \times 3$ - onda plana com incidência normal. a) Plano E: $E_{\theta}\left(\theta, \phi=0^{\circ}\right)$; b) Plano $\mathrm{H}: E_{\phi}\left(\theta, \phi=90^{\circ}\right)$. 
Capítulo 4. Análise de estruturas planares a partir do método dos momentos MoM

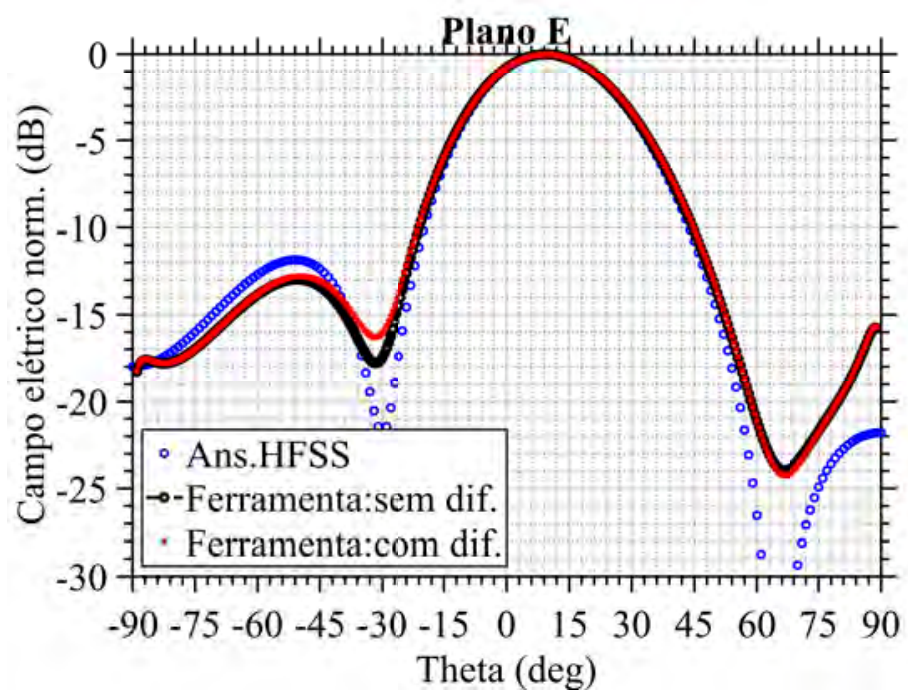

Figura 4.60: Campo calculado e simulado para uma rede $3 \times 3$ - onda plana com incidência oblíqua $\left(\theta_{i}=10^{\circ}\right)$. Plano E: $E_{\theta}\left(\theta, \phi=0^{\circ}\right)$.

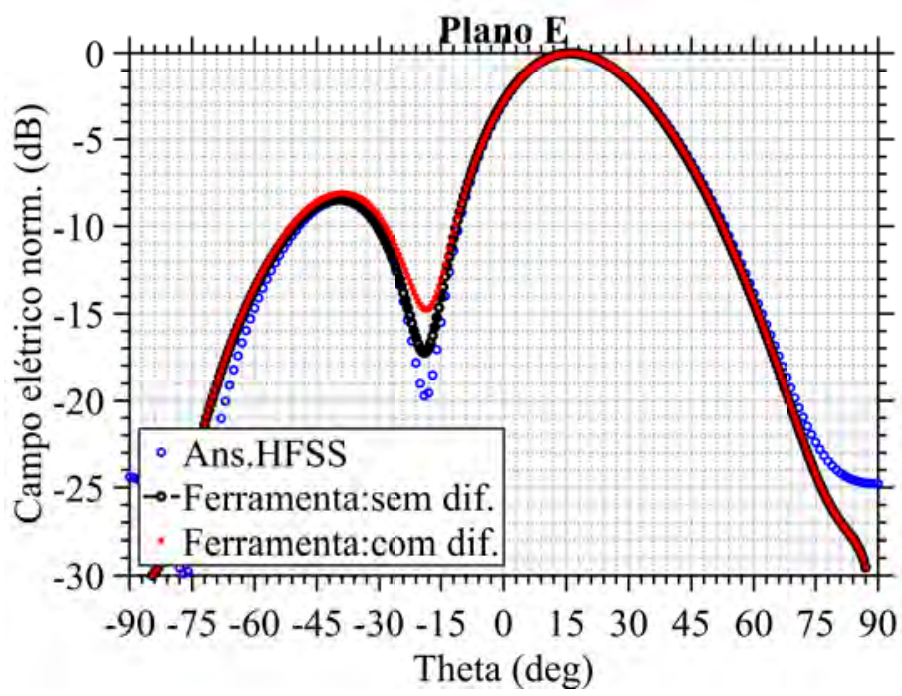

Figura 4.61: Campo calculado e simulado para uma rede $3 \times 3$ - onda plana com incidência oblíqua $\left(\theta_{i}=20^{\circ}\right)$. Plano E: $E_{\theta}\left(\theta, \phi=0^{\circ}\right)$. 
Capítulo 4. Análise de estruturas planares a partir do método dos momentos MoM

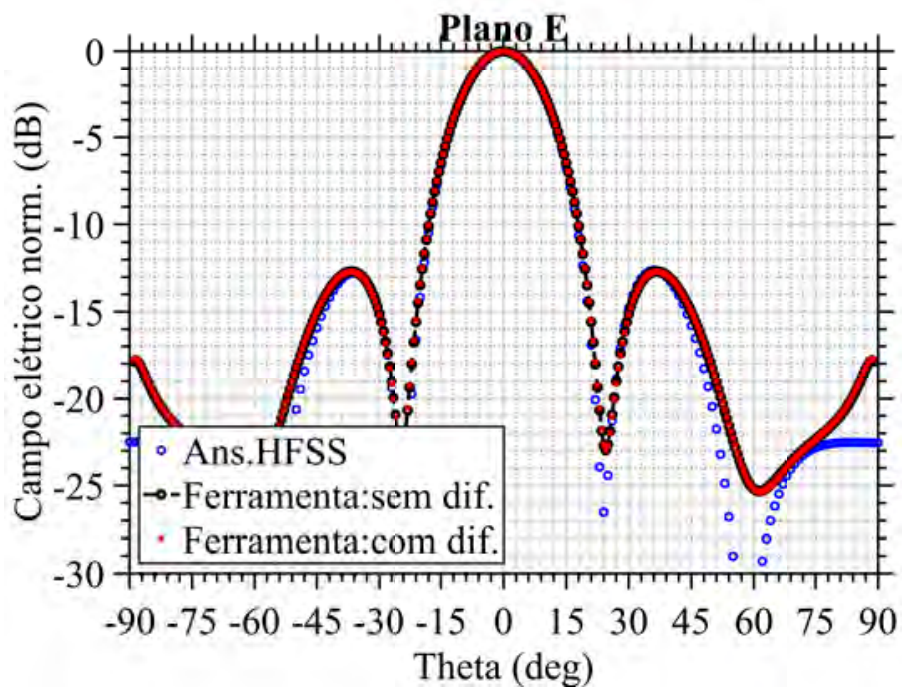

a)

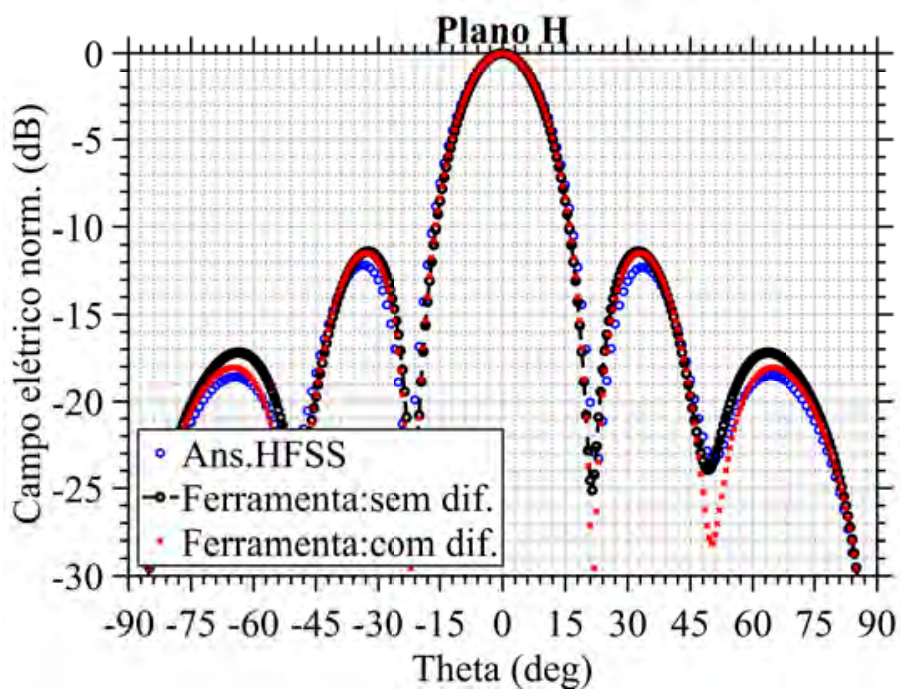

b)

Figura 4.62: Campo calculado e simulado para uma rede $5 \times 5$ - onda plana com incidência normal. a) Plano E: $E_{\theta}\left(\theta, \phi=0^{\circ}\right)$; b) Plano $\mathrm{H}: E_{\phi}\left(\theta, \phi=90^{\circ}\right)$. 
Capítulo 4. Análise de estruturas planares a partir do método dos momentos MoM

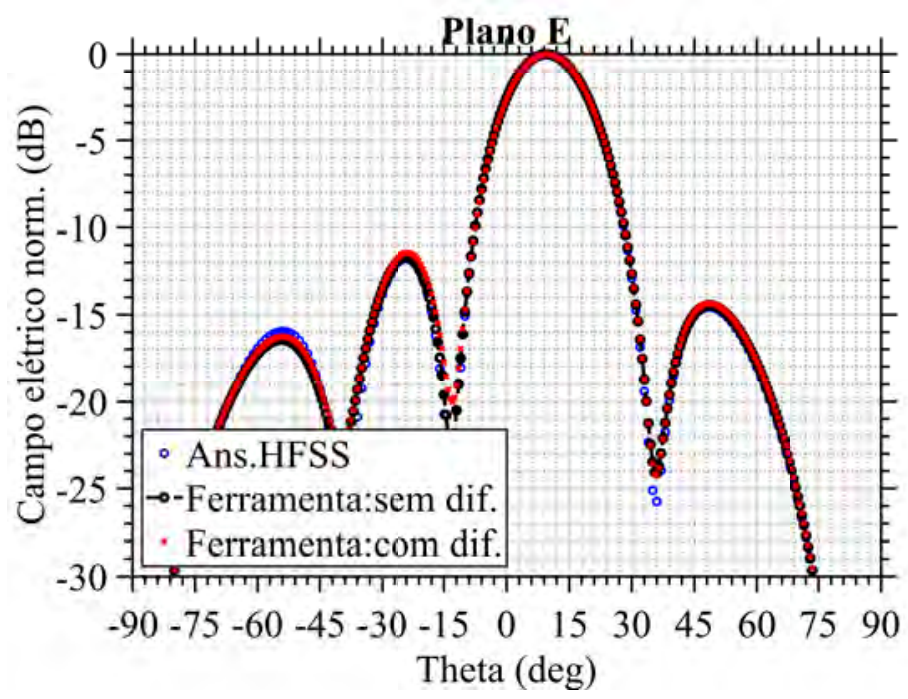

Figura 4.63: Campo calculado e simulado para uma rede $5 \times 5$ - onda plana com incidência oblíqua $\left(\theta_{i}=10^{\circ}\right)$. Plano E: $E_{\theta}\left(\theta, \phi=0^{\circ}\right)$.

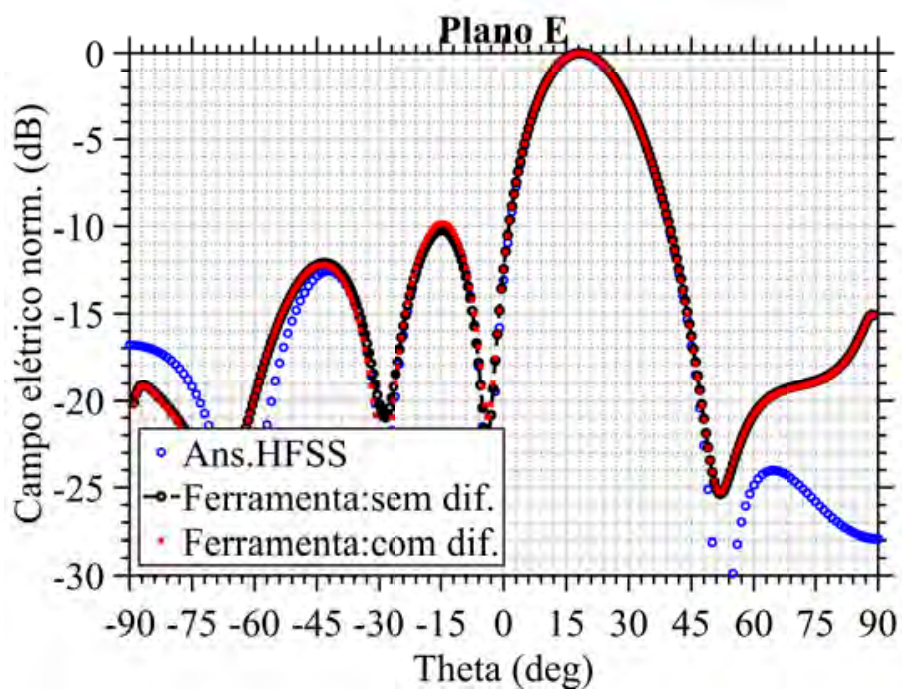

Figura 4.64: Campo calculado e simulado para uma rede $5 \times 5$ - onda plana com incidência oblíqua $\left(\theta_{i}=20^{\circ}\right)$. Plano E: $E_{\theta}\left(\theta, \phi=0^{\circ}\right)$. 
Capítulo 4. Análise de estruturas planares a partir do método dos momentos MoM

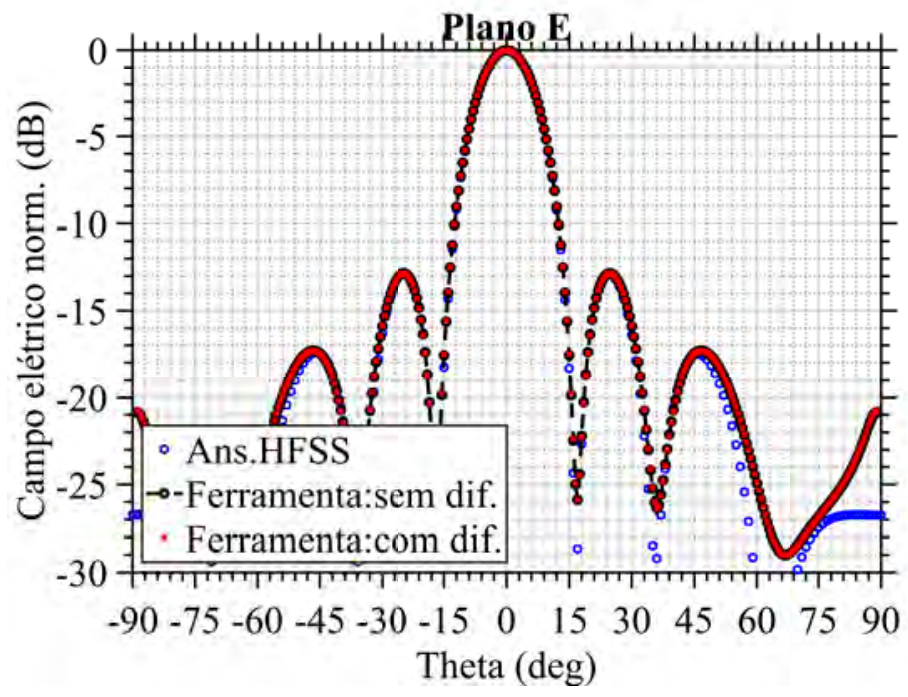

a)

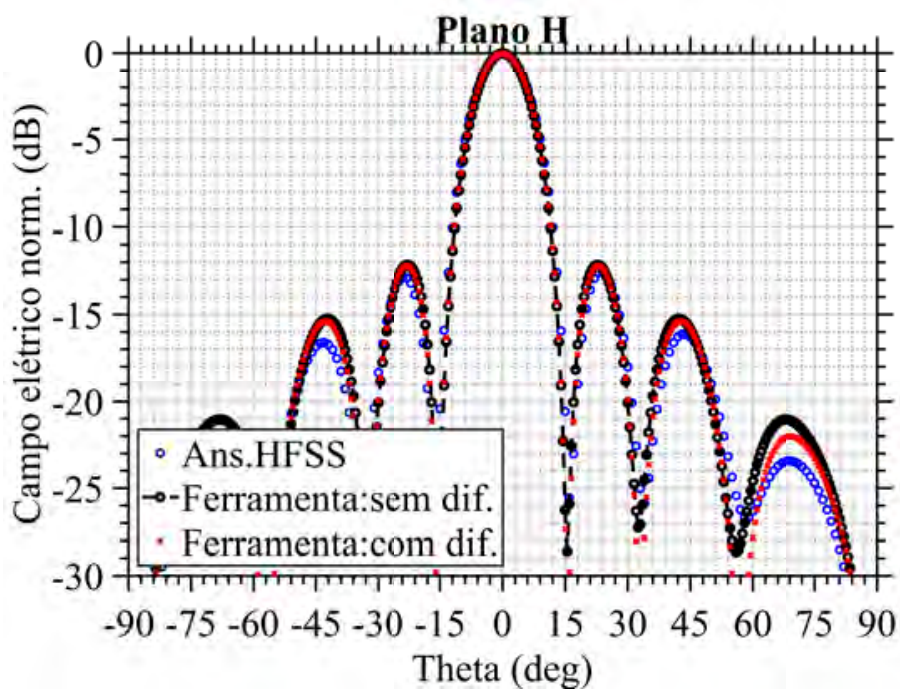

b)

Figura 4.65: Campo calculado e simulado para uma rede $7 \times 7$ - onda plana com incidência normal. a) Plano E: $E_{\theta}\left(\theta, \phi=0^{\circ}\right)$; b) Plano $\mathrm{H}: E_{\phi}\left(\theta, \phi=90^{\circ}\right)$. 
Capítulo 4. Análise de estruturas planares a partir do método dos momentos MoM

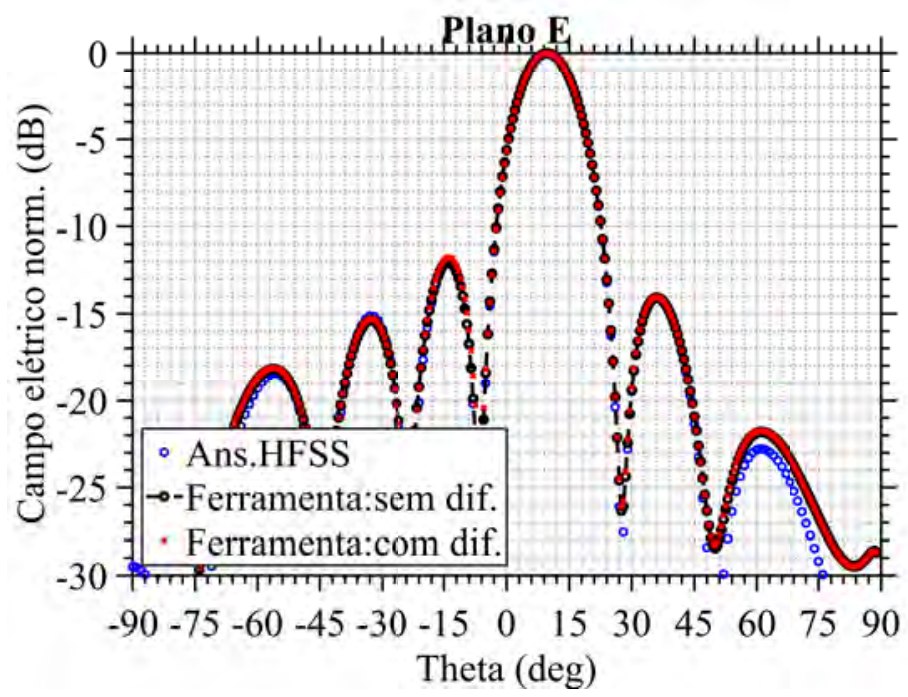

Figura 4.66: Campo calculado e simulado para uma rede $7 \times 7$ - onda plana com incidência oblíqua $\left(\theta_{i}=10^{\circ}\right)$. Plano E: $E_{\theta}\left(\theta, \phi=0^{\circ}\right)$.

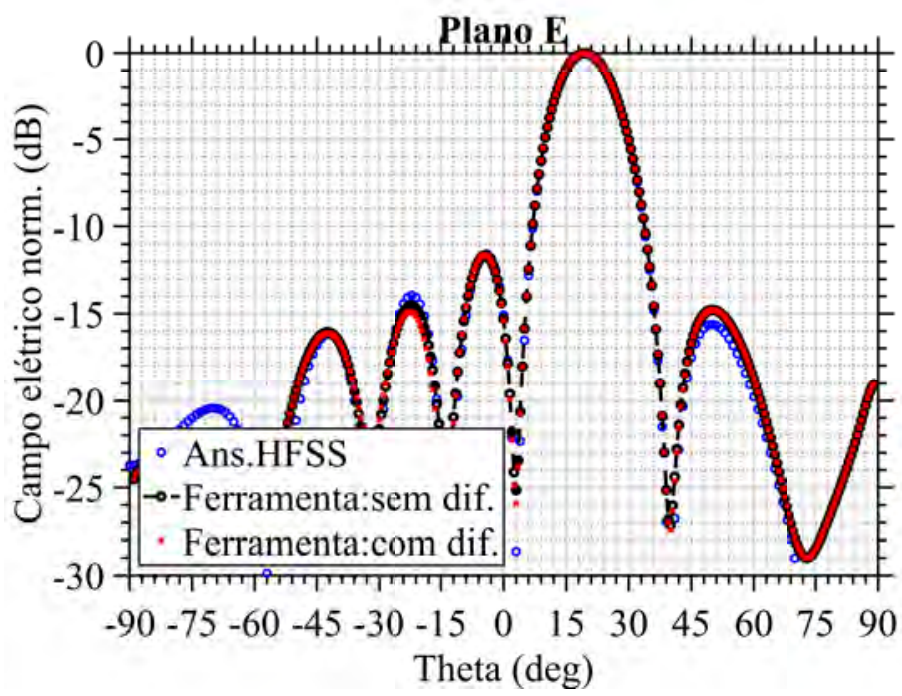

Figura 4.67: Campo calculado e simulado para uma rede $7 \times 7$ - onda plana com incidência oblíqua $\left(\theta_{i}=20^{\circ}\right)$. Plano E: $E_{\theta}\left(\theta, \phi=0^{\circ}\right)$. 


\section{5}

\section{Síntese de redes refletoras}

Neste capítulo é apresentado o formalismo da teoria e de síntese de diagramas de irradiação baseado na aplicação do conceito de defasagem progressiva e também de heurística para a determinação da fase desejada.

O campo elétrico produzido por uma rede de antenas avaliado em campo distante pode ser descrito da seguinte forma

$$
\begin{array}{r}
\mathbf{S}(r, \theta, \phi)=\frac{e^{-j k_{0} r}}{r} \sum_{u=1}^{E}\left[\left(E_{u_{\theta}}(\theta, \phi) \hat{\theta}+E_{u_{\phi}}(\theta, \phi) \hat{\phi}\right)\right. \\
\left.I_{u} e^{j k_{0}\left(x_{u}^{(0)} \operatorname{sen} \theta \cos \phi+y_{u}^{(0)} \operatorname{sen} \theta \operatorname{sen} \phi\right)}\right],
\end{array}
$$

em que $x_{u}^{(0)}$ e $y_{u}^{(0)}$ são as coordenadas dos elementos na rede refletora, $k_{0}=2 \pi / \lambda_{0}, E_{u_{\theta}}(\theta, \phi)$ e $E_{u_{\phi}}(\theta, \phi)$ são as componentes do campo elétrico complexo de cada célula nas coordenadas $\theta$ e $\phi$, e $I_{u}$ é um coeficiente complexo descrito na forma polar por

$$
I_{u}=A_{u} \angle \psi_{u}
$$

Em comparação às redes de antenas, pode-se dizer que os coeficientes de excitação, devido à fonte, estão suprimidos dentro dos campos irradiados pelas células que compõe uma rede refletora.

$\mathrm{Na}$ análise inicial foram avaliadas redes refletoras finitas compostas por elementos de mesmo tamanho físico, em que curvas de fase são obtidas a partir da avaliação da fase do campo elétrico total re-irradiado para a direção especular para cada célula a medida em que o tamanho dos patches quadrados são variados simultaneamente. Uma RA contém $E$ curvas de fase, sendo uma para cada célula. Os elementos relacionados por simetria na rede apresentam curvas de fase idênticas, conforme ilustrado nas Figuras 4.53, 4.54 e 4.55. As curvas iniciais apresentam 33 pontos discretos na faixa de 30 a $38 \mathrm{~mm}$ e, após interpoladas, um número de pontos 100 vezes superior.

No processo de síntese, a fase desejada em cada célula pode ser determinada analiticamente ou numericamente para prover a distribuição de fase desejada sobre a abertura. Desta forma, busca-se o diagrama de irradiação desejado. Após calculadas as fases para cada célula da rede, deve-se buscar o 
tamanho corresponde do espalhador na curva de fase individual para a respectiva dimensão da rede (exemplo: o tamanho do espalhador \#1 de uma RA $7 \times 7$, com base na fase calculada, é determinado a partir da curva de fase da célula \#1 de uma RA $7 \times 7$ ). Após obtido todos os tamanhos dos elementos, o leiaute da rede é exportado automaticamente, podendo-se importar na prototipadora de precisão e também nos softwares HFSS e Designer.

A estrutura projetada é novamente analisada para obter-se os diagramas de irradiação, visto que as curvas de fase utilizadas consideram elementos de mesmo tamanho físico, o que não é verdade após a definição de todos os tamanhos, o que pode provocar pequena mudança de fase. Por fim, a validação é realizada através dos resultados fornecidos pela ferramenta proposta e o software comercial. Todas as etapas aqui descritas são ilustradas na Figura 5.1.

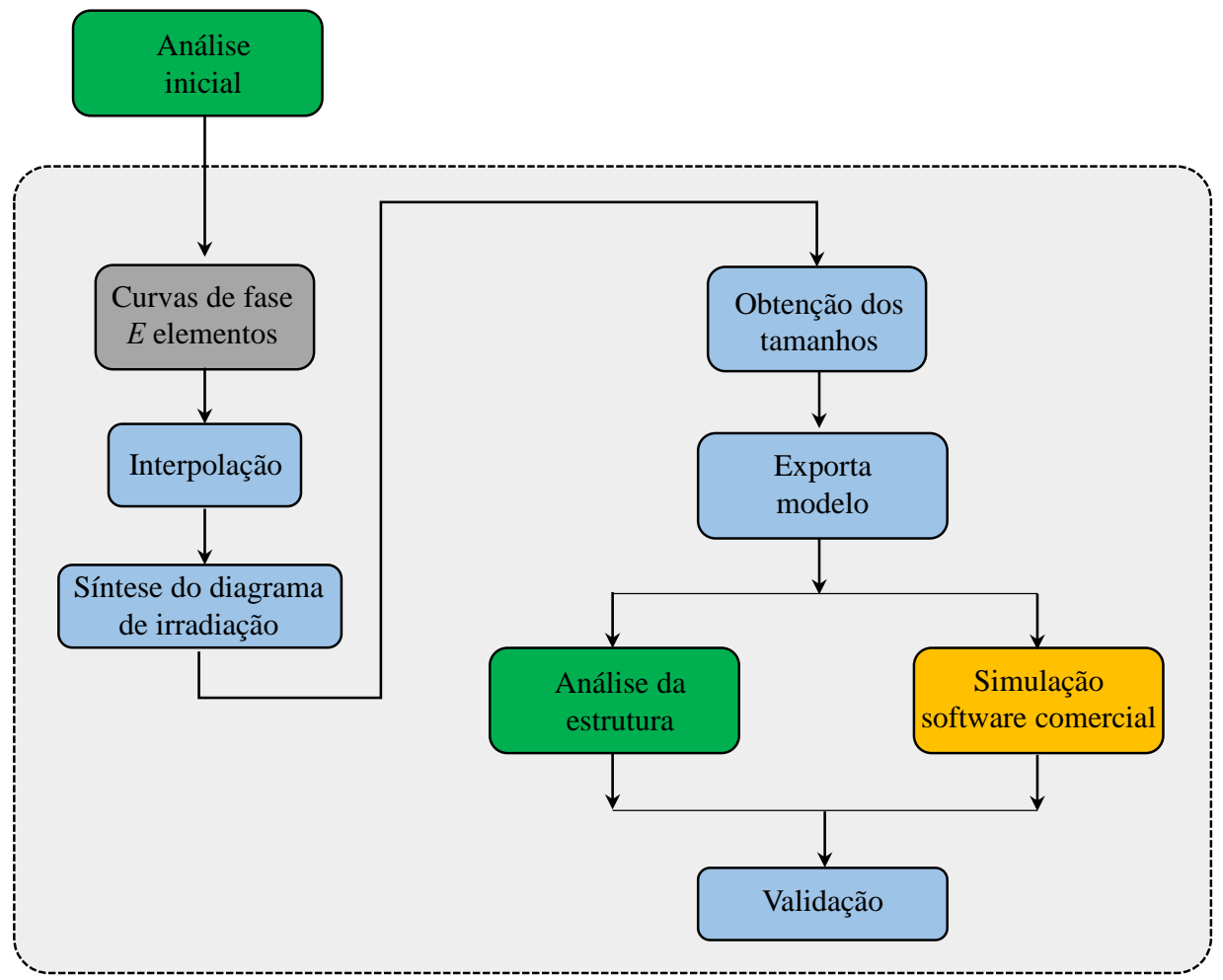

Figura 5.1: Fluxograma da síntese de diagramas de re-irradiação e validação.

Os casos abordados até o momento tratam-se de redes refletoras de dimensões $3 \times 3,5 \times 5$ e $7 \times 7$ com elementos de mesmo tamanho físico e diagrama de irradiação na direção especular. Devido à pouca flexibilidade de redes $3 \times 3$, apenas as demais dimensões são analisadas.

Inicialmente, realiza-se a síntese do diagrama de irradiação para projetar os tamanhos dos elementos para se obter o apontamento do feixe para determinada direção. Para este caso, aplica-se a defasagem progressiva entre os elementos da rede para determinar analiticamente a fase desejada. Pos- 
teriormente, o método de enxame de partículas é utilizado para determinar numericamente a fase para reproduzir um diagrama com feixe retangular nos planos E e H.

\section{1}

\section{Síntese de rede refletora com apontamento de feixe}

Para a incidência de uma onda plana sobre uma rede refletora, tem-se o feixe principal na direção especular quando todos elementos da rede possuírem o mesmo tamanho físico, ou seja, observa-se o apontamento para $20^{\circ}$ apenas quando a incidência ocorrer com a respectiva inclinação.

Aqui, realiza-se o apontamento do diagrama de irradiação para uma direção diferente da especular. Esse apontamento é realizado diferentemente do conceito de tilt, que é dado mecanicamente. Em comparação com os refletores clássicos, poder-se-ia dizer que é equivalente a modelar a estrutura metálica do refletor. O caso analisado trata-se de uma onda plana com incidência normal, linearmente polarizada em $x$ e direcionamento do feixe para $20^{\circ}$.

Diagramas de irradiação com apontamento de feixe podem ser obtidos através do conceito de defasagem progressiva entre o centro dos patches para calcular analiticamente as fases desejadas, sendo, para este caso, determinadas como sendo [2],

$$
\psi_{u}=-k_{0}\left[x_{u}^{(0)} \operatorname{sen} \theta_{b} \cos \phi_{b}+y_{u}^{(0)} \operatorname{sen} \theta_{b} \operatorname{sen} \phi_{b}\right],
$$

em que $\theta_{b}$ e $\phi_{b}$ definem a direção do feixe principal.

Nas Tabelas 5.1, 5.2 e 5.3 e nas Tabelas $5.4,5.5$ e 5.6 são apresentadas as numerações das células na rede, as fases desejadas (determinadas a partir da (5-3)) e as dimensões correspondentes para redes $5 \times 5$ e $7 \times 7$, respectivamente.

Tabela 5.1: Numerações das células de uma rede refletora $5 \times 5$.

\begin{tabular}{|c|c|c|c|c|}
\hline 21 & 22 & 23 & 24 & 25 \\
\hline 16 & 17 & 18 & 19 & 20 \\
\hline 11 & 12 & 13 & 14 & 15 \\
\hline 6 & 7 & 8 & 9 & 10 \\
\hline 1 & 2 & 3 & 4 & 5 \\
\hline
\end{tabular}


Tabela 5.2: Fases que devem ser reproduzidas pelas células em graus $\left(^{\circ}\right)$.

\begin{tabular}{|l|l|l|l|l|}
\hline 123,2 & 61,6 & 0,0 & $-61,6$ & $-123,2$ \\
\hline 123,2 & 61,6 & 0,0 & $-61,6$ & $-123,2$ \\
\hline 123,2 & 61,6 & 0,0 & $-61,6$ & $-123,2$ \\
\hline 123,2 & 61,6 & 0,0 & $-61,6$ & $-123,2$ \\
\hline 123,2 & 61,6 & 0,0 & $-61,6$ & $-123,2$ \\
\hline
\end{tabular}

Tabela 5.3: Tamanhos dos patches quadrados em milímetros.

\begin{tabular}{|c|c|c|c|c|}
\hline 33,27 & 33,75 & 33,94 & 34,18 & 34,59 \\
\hline 33,29 & 33,76 & 34,02 & 34,23 & 34,67 \\
\hline 33,3 & 33,73 & 33,95 & 34,19 & 34,64 \\
\hline 33,29 & 33,76 & 34,02 & 34,23 & 34,67 \\
\hline 33,27 & 33,75 & 33,94 & 34,18 & 34,59 \\
\hline
\end{tabular}

Tabela 5.4: Numerações das células de uma rede refletora $7 \times 7$.

\begin{tabular}{|c|c|c|c|c|c|c|}
\hline 43 & 44 & 45 & 46 & 47 & 48 & 49 \\
\hline 36 & 37 & 38 & 39 & 40 & 41 & 42 \\
\hline 29 & 30 & 31 & 32 & 33 & 34 & 35 \\
\hline 22 & 23 & 24 & 25 & 26 & 27 & 28 \\
\hline 15 & 16 & 17 & 18 & 19 & 20 & 21 \\
\hline 8 & 9 & 10 & 11 & 12 & 13 & 14 \\
\hline 1 & 2 & 3 & 4 & 5 & 6 & 7 \\
\hline
\end{tabular}

Tabela 5.5: Fases que devem ser reproduzidas pelas células em graus $\left(^{\circ}\right)$.

\begin{tabular}{|l|l|l|l|l|l|l|}
\hline 184,7 & 123,2 & 61,6 & 0,0 & $-61,6$ & $-123,2$ & $-184,7$ \\
\hline 184,7 & 123,2 & 61,6 & 0,0 & $-61,6$ & $-123,2$ & $-184,7$ \\
\hline 184,7 & 123,2 & 61,6 & 0,0 & $-61,6$ & $-123,2$ & $-184,7$ \\
\hline 184,7 & 123,2 & 61,6 & 0,0 & $-61,6$ & $-123,2$ & $-184,7$ \\
\hline 184,7 & 123,2 & 61,6 & 0,0 & $-61,6$ & $-123,2$ & $-184,7$ \\
\hline 184,7 & 123,2 & 61,6 & 0,0 & $-61,6$ & $-123,2$ & $-184,7$ \\
\hline 184,7 & 123,2 & 61,6 & 0,0 & $-61,6$ & $-123,2$ & $-184,7$ \\
\hline
\end{tabular}

Tabela 5.6: Tamanhos dos patches quadrados em milímetros.

\begin{tabular}{|l|l|l|l|l|l|l|}
\hline 30,00 & 33,32 & 33,75 & 33,94 & 34,17 & 34,68 & 38,00 \\
\hline 30,00 & 33,35 & 33,78 & 33,99 & 34,21 & 34,76 & 38,00 \\
\hline 30,00 & 33,34 & 33,78 & 33,94 & 34,18 & 34,74 & 38,00 \\
\hline 30,00 & 33,32 & 33,74 & 33,97 & 34,21 & 34,74 & 38,00 \\
\hline 30,00 & 33,34 & 33,78 & 33,94 & 34,18 & 34,74 & 38,00 \\
\hline 30,00 & 33,35 & 33,78 & 33,99 & 34,21 & 34,76 & 38,00 \\
\hline 30,00 & 33,32 & 33,75 & 33,94 & 34,17 & 34,68 & 38,00 \\
\hline
\end{tabular}


Os leiautes gerados pela ferramenta para ambas as redes, conforme dimensões contidas nas Tabelas 5.3 e 5.6, podem ser visualizados nas Figuras 5.2 e 5.3 .

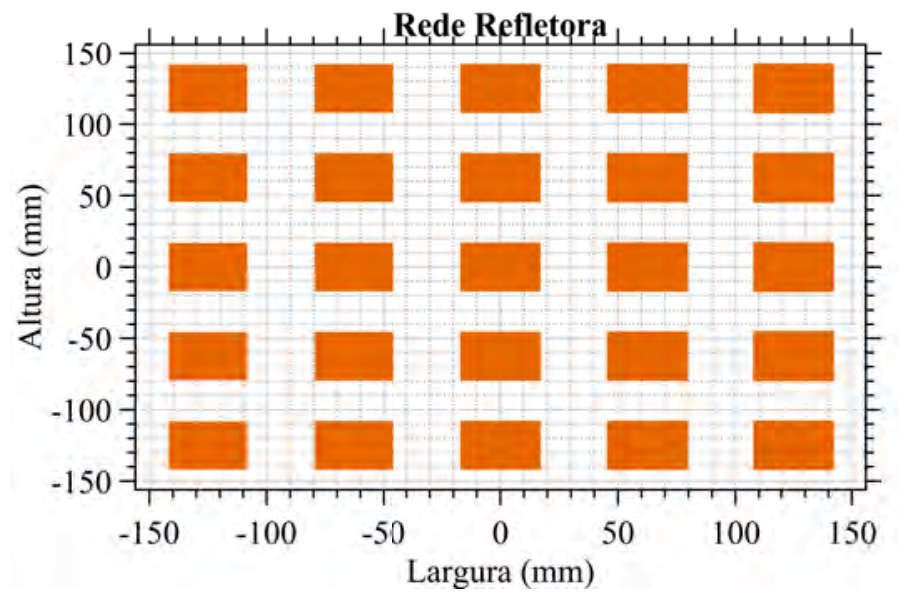

Figura 5.2: Rede $5 \times 5$ com elementos de dimensões físicas apresentadas na Tabela 5.3.

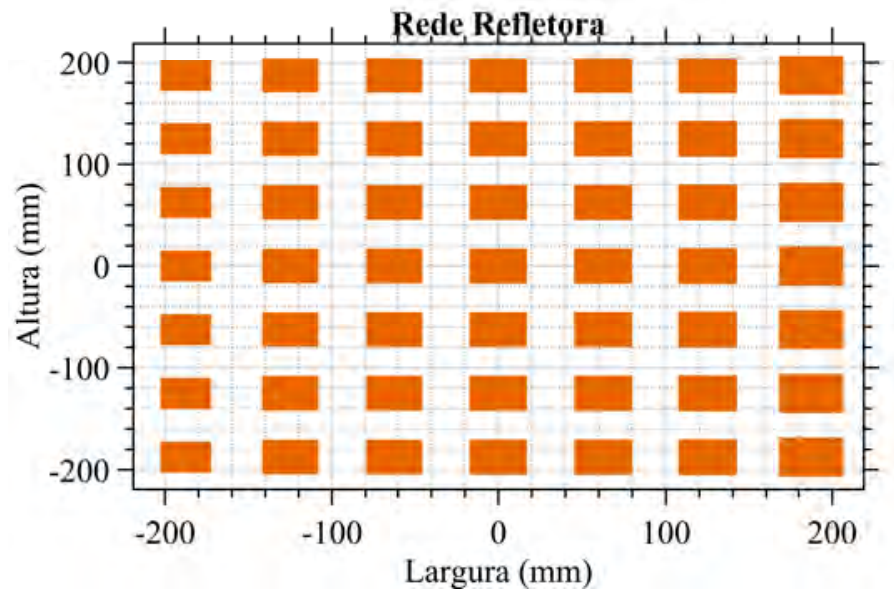

Figura 5.3: Rede $7 \times 7$ com elementos de dimensões físicas apresentadas na Tabela 5.6.

Os resultados obtidos são apresentados nas Figuras 5.4 e 5.5, em que percebe-se a direção de apontamento conforme o especificado. Adicionalmente, constata-se que a posição dos nulos coincidem, sendo assim, pode-se afirmar que as fases calculadas são corretamente reproduzidas pelos elementos. Entretanto, verifica-se que o nível dos lóbulos laterais contém diferença para o software de verificação. Esta discrepância está relacionada com o erro na amplitude individual de alguns elementos que compõe a rede refletora. 


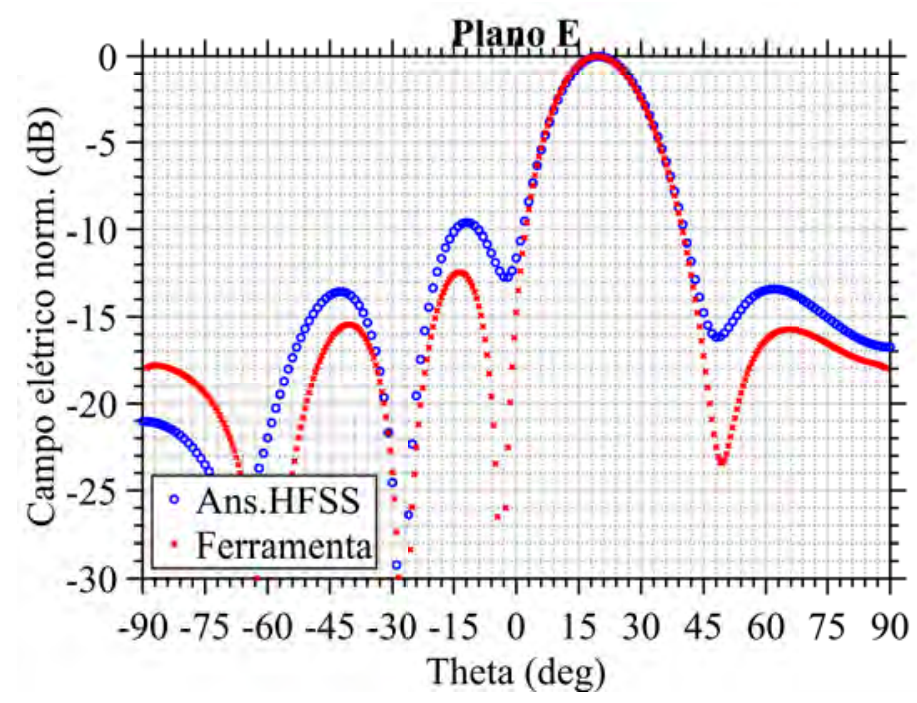

Figura 5.4: Campo calculado e simulado para uma rede $5 \times 5$ - onda plana com incidência normal. Plano E: $E_{\theta}\left(\theta, \phi=0^{\circ}\right)$.

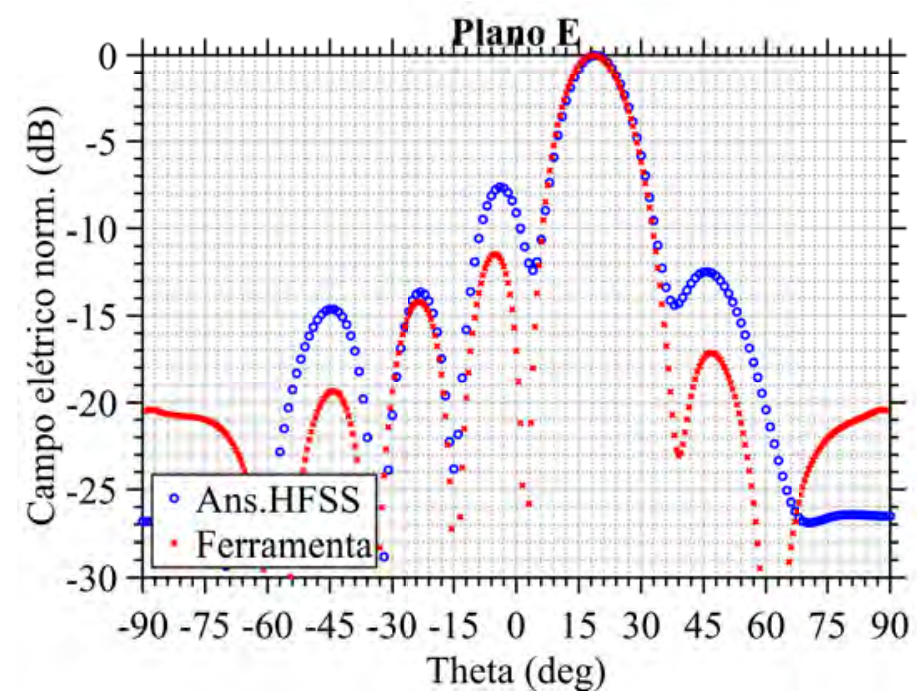

Figura 5.5: Campo calculado e simulado para uma rede $7 \times 7$ - onda plana com incidência normal. Plano E: $E_{\theta}\left(\theta, \phi=0^{\circ}\right)$.

Para comprovar tal afirmação, pode-se observar na Figura 4.19 um pequeno erro de amplitude entre o campo espalhado calculado e o Designer para dimensões mais afastadas da ressonância, que neste caso afeta a rede $5 \times 5$ pelas células contidas nas colunas 1 e 5 da Tabela 5.3 e para a rede $7 \times 7$ pelas células contidas nas colunas 1, 2, 6 e 7 da Tabela 5.6.

Nas Figuras 5.6 e 5.7 são ilustrados os resultados após mudança pontual de $2 \%$ na amplitude das células citadas. Pode-se verificar que a resposta aproximou melhor o resultado obtido pela ferramenta comercial. Como alternativa para a completa correção, deve-se aprimorar a representação da função 
base com borda segmentada para dimensões mais afastadas da ressonância, de forma a corrigir o erro de amplitude.

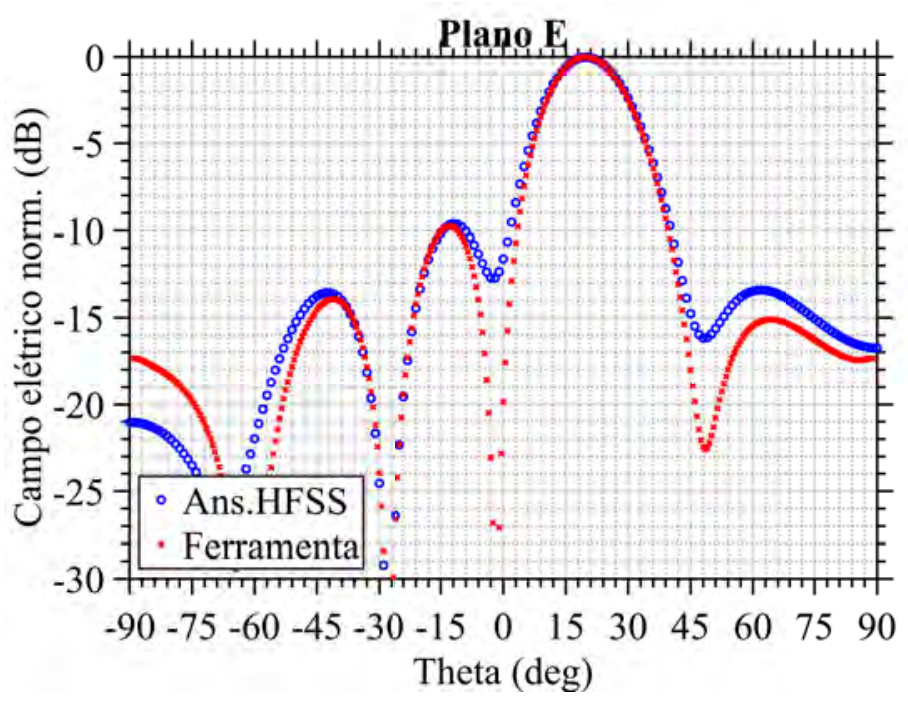

Figura 5.6: Campo calculado e simulado para uma rede $5 \times 5$ após correção pontual nas amplitudes dos campos elétricos dos patches - onda plana com incidência normal. Plano $\mathrm{E}: E_{\theta}\left(\theta, \phi=0^{\circ}\right)$.

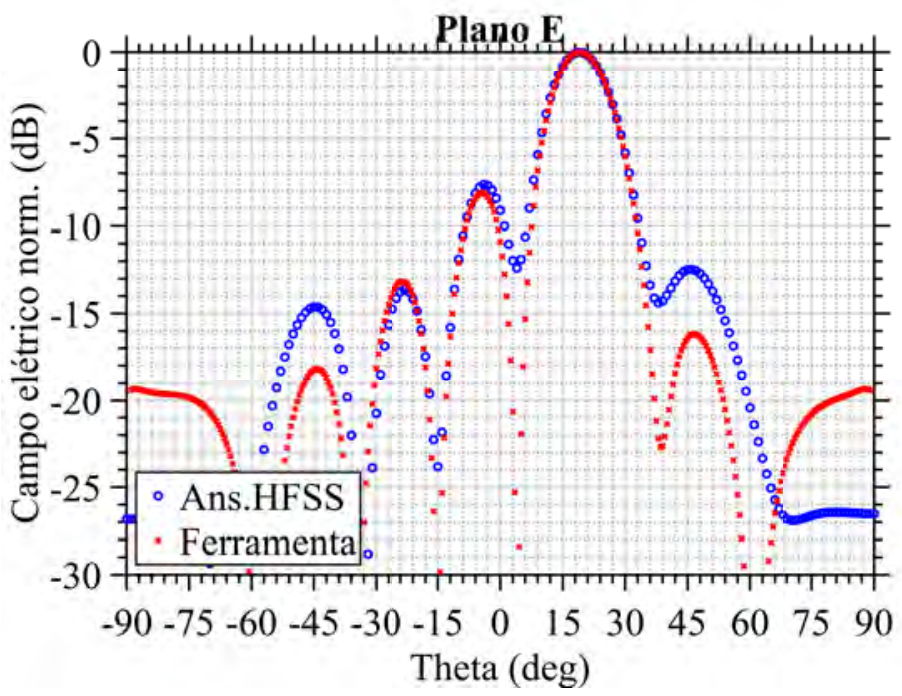

Figura 5.7: Campo calculado e simulado para uma rede $7 \times 7$ após correção pontual nas amplitudes dos campos elétricos dos patches - onda plana com incidência normal . Plano E: $E_{\theta}\left(\theta, \phi=0^{\circ}\right)$.

Nas Figuras 5.8 e 5.9 são ilustrados o diagrama de irradiação 3D com contorno e a vista isométrica do campo elétrico tridimensional re-irradiado no software HFSS para as redes projetadas a partir da modelagem eletromagnética descrita neste trabalho. 


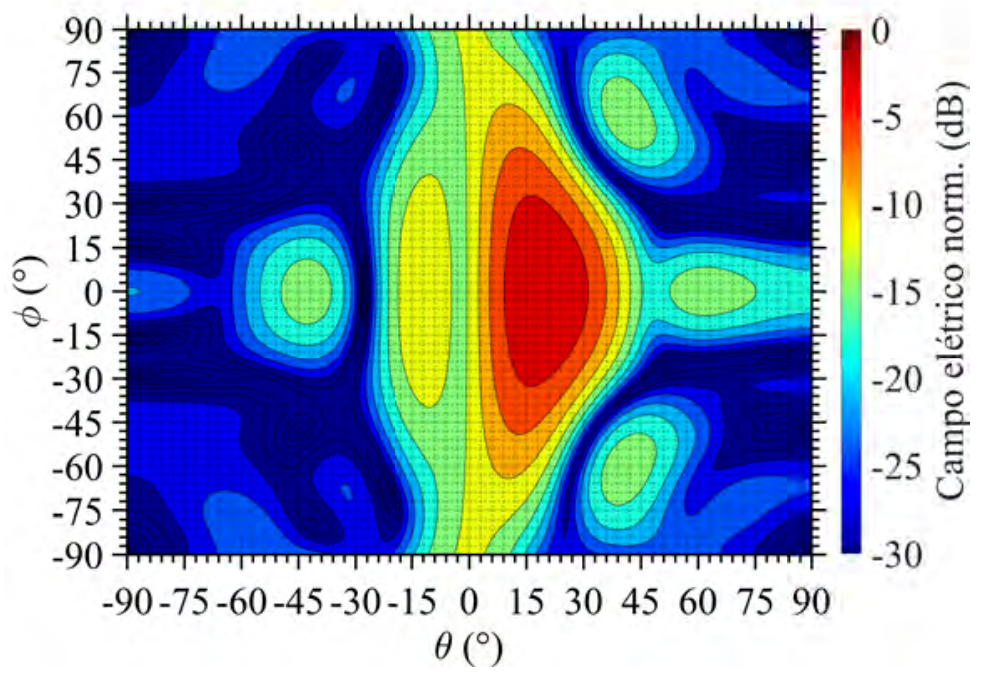

a)

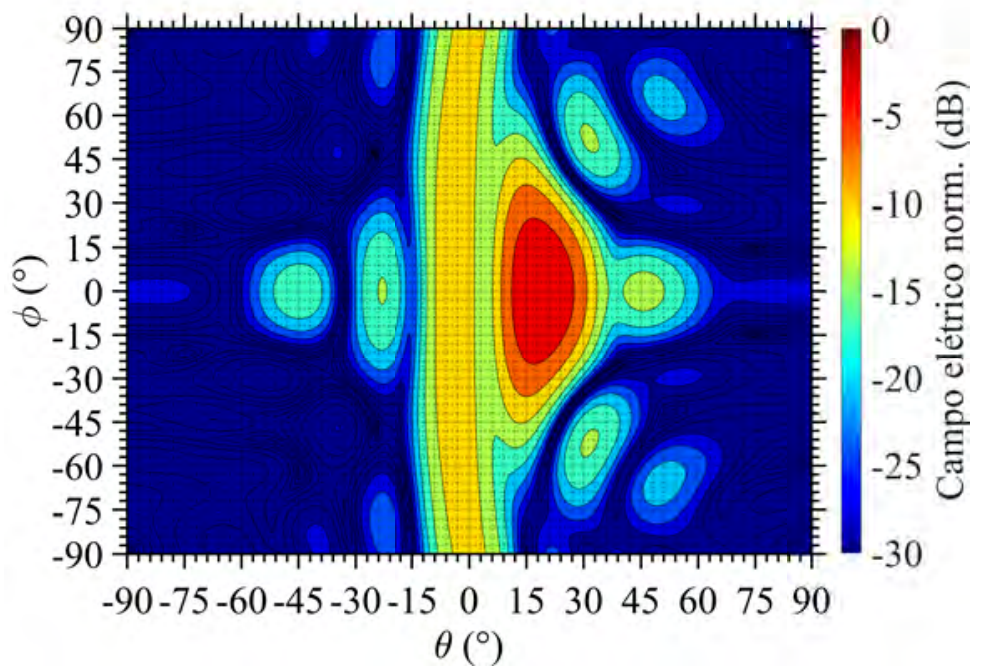

b)

Figura 5.8: Diagramas de re-irradiação com contorno. a) RA $5 \times 5$; b) RA $7 \times 7$.

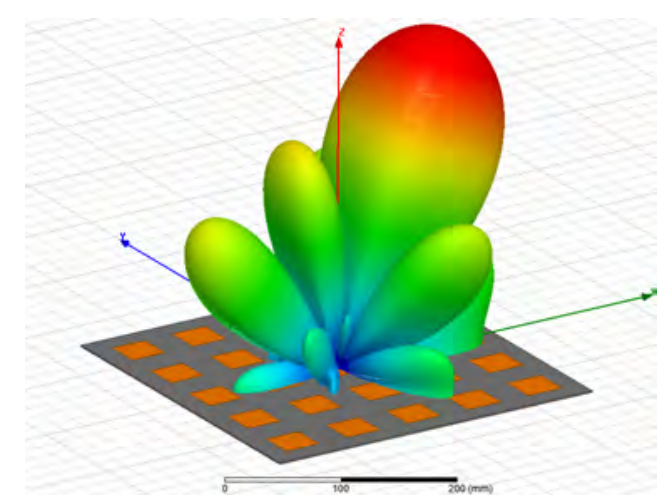

a)

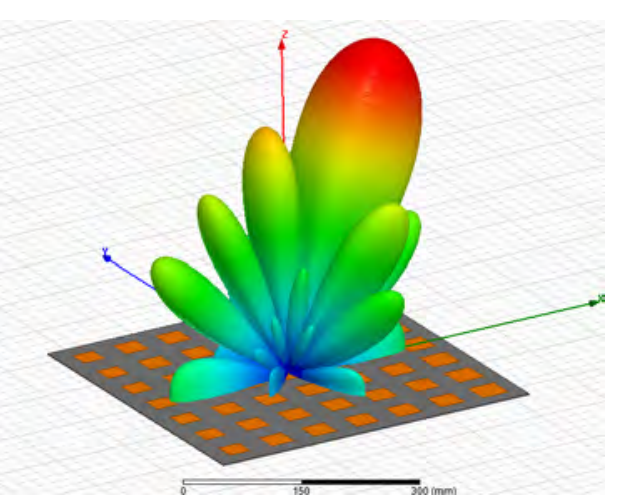

b)

Figura 5.9: Diagramas de re-irradiação 3D com apontamento para $\theta_{b}=20^{\circ}$ e $\phi_{b}=0^{\circ}$. a) RA $5 \times 5$; b) RA $7 \times 7$. 


\section{2}

\section{Síntese de rede refletora com feixe conformado}

Tratando-se de redes refletoras, pode-se dizer que as fases são difíceis de serem calculadas analiticamente para diagramas com formato complexo, sendo assim, conveniente o emprego de técnicas apropriadas. Neste trabalho, o método de otimização por enxame de partículas (PSO) é utilizado na síntese de um diagrama de irradiação com feixe retangular nos planos E e H. A técnica é aplicada na definição da fase desejada em cada célula, sendo assim, possível a determinação do tamanho de cada metalização impressa. O diagrama foi sintetizado considerando elementos irradiando com a mesma intensidade.

Para diagramas com simetria, tais como isoflux e retangular (com linha de simetria na direção broadside), as características da rede podem ser exploradas para minimizar o número de variáveis a serem calculadas numericamente. Adicionalmente, apenas é necessário determinar o plano $\mathrm{E}$ da rede, sendo o plano $\mathrm{H}$ obtido simultaneamente devido à simetria.

O método de enxame de partículas é um algoritmo evolucionário baseado no comportamento de seres vivos na natureza, como, por exemplo, os pássaros. As aves sobrevoam lugares em busca de alimentos, assim suas posições e velocidades mudam no ambiente ao longo do tempo. A interação entre elas acontece quando as aves encontram um lugar com alta concentração de alimento e, posteriormente, ocorre o deslocamento para o local de interesse. Essa técnica consiste em três conceitos, que são: o movimento das aves, a memória individual dos lugares que contém alimento, e a interação do bando, cuja a localização com maior concentração de mantimento é informada entre as aves [108].

O comportamento descrito anteriormente pode ser convertido em um problema de otimização. Para isso, definem-se algumas relações, como apresentadas na Tabela 5.7.

O algoritmo de otimização deve encontrar a fase e a amplitude do campo de cada elemento que compõe a rede refletora para sintetizar um diagrama específico. O PSO é inicializado com uma geração randômica de partículas dentro do espaço de busca. As partículas geradas possuem todas as variáveis que se deseja otimizar. Neste caso, as amplitudes e as fases do campo elétrico produzido por cada divisão da RA. Essa técnica consiste na atualização iterativa da posição de cada partícula através da seguinte representação,

$$
\nu_{n}^{k}=w_{i} \nu_{n-1}^{k}+C_{1} \operatorname{rand}(.)\left(p_{\text {best }}^{k}-p_{n-1}^{k}\right)+C_{2} \operatorname{rand}(.)\left(g_{\text {best }}-p_{n-1}^{k}\right),
$$

com

$$
p_{n}^{k}=p_{n-1}^{k}+\nu_{n}^{k},
$$


Tabela 5.7: Variáveis que relacionam o algoritmo de otimização com a natureza.

\begin{tabular}{|l|l|}
\hline Algoritmo & Natureza \\
\hline Partícula & Ave \\
\hline Enxame & Bando \\
\hline Espaço de busca & Espaço limitado de voo que contém alimento \\
\hline Melhor solução local & $\begin{array}{l}\text { Melhor lugar encontrado por uma ave dentro } \\
\text { de um espaço limitado }\end{array}$ \\
\hline Melhor solução global & $\begin{array}{l}\text { Melhor lugar encontrado pelo bando dentro } \\
\text { de um espaço limitado }\end{array}$ \\
\hline $\begin{array}{l}\text { Constante de confiança da } \\
\text { partícula em si mesmo }\end{array}$ & Auto-confiança da própria ave \\
\hline $\begin{array}{l}\text { Constante de confiança da } \\
\text { partícula no enxame }\end{array}$ & Confiança no bando \\
\hline Inércia da partícula & $\begin{array}{l}\text { Continuidade na direção de deslocamento da } \\
\text { ave }\end{array}$ \\
\hline
\end{tabular}

em que $w_{i}$ é a inércia, $C_{1}$ e $C_{2}$ são coeficientes constantes associados com o quanto a partícula confia em si própria e quanto confia no enxame, respectivamente. Os termos $\nu_{n-1}^{k}$ e $\nu_{n}^{k}$ são a última e atual velocidades, $p_{n-1}^{k}$ e $p_{n}^{k}$ são a última e atual posições de uma $k$-ésima partícula, rand(.) é um valor escalar randômico entre 0 e $1, p_{\text {best }}^{k}$ é a melhor posição encontrada pela $k$-ésima partícula e $g_{\text {best }}$ é a melhor posição encontrada pelo enxame.

$\mathrm{Na}$ otimização de uma rede refletora com PSO, uma partícula contém um conjunto de coeficientes necessário para sintetizar um diagrama desejado. Na forma matricial, (5-4) e (5-5) podem ser reescritas como

$$
\mathbf{V}_{n}^{k}=w_{i} \mathbf{V}_{n-1}^{k}+C_{1} \operatorname{rand}(.)\left(\mathbf{P}_{\text {best }}^{k}-\mathbf{P}_{n-1}^{k}\right)+C_{2} \operatorname{rand}(.)\left(\mathbf{G}_{\text {best }}-\mathbf{P}_{n-1}^{k}\right)
$$

e

$$
\mathbf{P}_{n}^{k}=\mathbf{P}_{n-1}^{k}+\mathbf{V}_{n}^{k}
$$

sendo $\mathbf{P}_{n}^{k}$ e $\mathbf{V}_{n}^{k}$ os vetores descritos para essa aplicação por,

$$
\mathbf{P}_{n-1}^{k}=\left[\begin{array}{llllllllll}
A_{1} & \psi_{1} & A_{2} & \psi_{2} & \ldots & A_{\varsigma} & \psi_{\varsigma} & \ldots & A_{E} & \psi_{E}
\end{array}\right]
$$

e

$$
\mathbf{V}_{n}^{k}=\left[\begin{array}{llllllll}
\Delta A_{1} & \Delta \psi_{1} & \Delta A_{2} & \Delta \psi_{2} & \ldots \Delta A_{\varsigma} & \Delta \psi_{\varsigma} & \ldots \Delta A_{E} & \Delta \psi_{E}
\end{array}\right],
$$

em que $\mathbf{V}_{n-1}^{k}$ e $\mathbf{V}_{n}^{k}$ são o último e atual vetores velocidade, e $\mathbf{P}_{n-1}^{k}$ e $\mathbf{P}_{n}^{k}$ são o último e atual vetores posição de uma $k$-ésima partícula de $E$ patches. Vale salientar que cada valor da partícula corresponde a uma dimensão no espaço de busca. Após a última iteração, os valores do vetor $\mathbf{G}_{\text {best }}$ correspondem às amplitudes e às fases desejadas para os elementos da RA.

Apesar da abordagem completa ser apresentada, a maioria dos trabalhos 
concentram-se na otimização da fase apenas, sendo, neste caso, a amplitude suprimida das expressões aplicadas no processo de síntese [6].

As partículas contendo as variáveis desejadas passam por um processo de avaliação, denominado de fitness, em que o erro entre os diagramas desejado e calculado é avaliado, sendo, a partir disso, informado quanto a partícula é boa como solução do problema. A partir da interação entre as demais partículas, o processo iterativo faz com que as partículas se desloquem para uma ótima solução global [109].

A avaliação discreta, definida como função objetivo, é realizada fazendo uso da (5-10),

$$
\text { fitness }=\sum_{g=1}^{2}\left[\frac{\xi_{g}}{N_{g}} \sum\left|\mathbf{S}\left(r, \theta_{v}, \phi_{o}\right)-\mathbf{E}\left(r, \theta_{v}, \phi_{o}\right)\right|\right],
$$

em que $\xi_{g}$ denota o peso e $N_{g}$ representa o número total de pontos na $g$ ésima região, com $g=1$ para o feixe principal e $g=2$ para a região dos lóbulos laterais. Os índices $v$ e $o$ denotam os pontos discretos de avaliação nas coordenadas $\theta$ e $\phi$, respectivamente.

Os feixes retangulares sintetizados devem ter abertura de $60^{\circ}$ e $40^{\circ}$, com ripple máximo permitido de $3 \mathrm{~dB}$, enquanto que as regiões de transição são definidas entre $\{-75$ a -40 e 40 a 75$\}$ e $\{-40$ a -25 e 25 a 40$\}$ para as redes $5 \times 5$ e $7 \times 7$, respectivamente. Já os lóbulos laterais devem ser minimizados, buscando um nível abaixo de -20 dB. Nas Tabelas 5.8 e 5.9 e nas Tabelas 5.10 e 5.11 são apresentadas as fases desejadas (determinadas a partir do PSO) e as dimensões correspondentes para redes $5 \times 5$ e $7 \times 7$.

Tabela 5.8: Fases que devem ser reproduzidas pelas células em graus $\left(^{\circ}\right)$.

\begin{tabular}{|c|c|c|c|c|}
\hline 110,49 & $-114,17$ & $-11,52$ & $-114,17$ & 110,49 \\
\hline$-114,17$ & 64,51 & 28,24 & 64,51 & $-114,17$ \\
\hline$-11,52$ & 28,24 & 137,12 & 28,24 & $-11,52$ \\
\hline$-114,17$ & 64,51 & 28,24 & 64,51 & $-114,17$ \\
\hline 110,49 & $-114,17$ & $-11,52$ & $-114,17$ & 110,49 \\
\hline
\end{tabular}

Tabela 5.9: Tamanhos dos patches quadrados em milímetros.

\begin{tabular}{|l|l|l|l|l|}
\hline 33,33 & 34,48 & 33,96 & 34,48 & 33,33 \\
\hline 34,46 & 33,71 & 33,88 & 33,71 & 34,46 \\
\hline 33,92 & 33,82 & 32,99 & 33,82 & 33,92 \\
\hline 34,46 & 33,71 & 33,88 & 33,71 & 34,46 \\
\hline 33,33 & 34,48 & 33,96 & 34,48 & 33,33 \\
\hline
\end{tabular}


Tabela 5.10: Fases que devem ser reproduzidas pelas células em graus $\left(^{\circ}\right)$.

\begin{tabular}{|c|c|c|c|c|c|c|}
\hline$-79,21$ & 148,87 & 88,05 & $-46,44$ & 88,05 & 148,87 & $-79,21$ \\
\hline 148,87 & $-153,75$ & 28,06 & 20,54 & 28,06 & $-153,75$ & 148,87 \\
\hline 88,05 & 28,06 & $-92,7$ & $-19,85$ & $-92,7$ & 28,06 & 88,05 \\
\hline$-46,44$ & 20,54 & $-19,85$ & 48,88 & $-19,85$ & 20,54 & $-46,43$ \\
\hline 88,05 & 28,06 & $-92,7$ & $-19,85$ & $-92,7$ & 28,06 & 88,05 \\
\hline 148,87 & $-153,75$ & 28,06 & 20,54 & 28,06 & $-153,75$ & 148,87 \\
\hline$-79,21$ & 148,87 & 88,05 & $-46,44$ & 88,05 & 148,87 & $-79,21$ \\
\hline
\end{tabular}

Tabela 5.11: Tamanhos dos patches quadrados em milímetros.

\begin{tabular}{|l|l|l|l|l|l|l|}
\hline 34,18 & 32,41 & 33,57 & 34,09 & 33,57 & 32,41 & 34,18 \\
\hline 32,55 & 35,53 & 33,88 & 33,87 & 33,88 & 35,53 & 32,55 \\
\hline 33,56 & 33,83 & 34,31 & 33,98 & 34,31 & 33,83 & 33,56 \\
\hline 34,08 & 33,88 & 34,04 & 33,77 & 34,04 & 33,88 & 34,08 \\
\hline 33,56 & 33,83 & 34,31 & 33,98 & 34,31 & 33,83 & 33,56 \\
\hline 32,55 & 35,53 & 33,88 & 33,87 & 33,88 & 35,53 & 32,55 \\
\hline 34,18 & 32,41 & 33,57 & 34,09 & 33,57 & 32,41 & 34,18 \\
\hline
\end{tabular}

Os resultados obtidos são apresentados nas Figuras 5.10 e 5.11, em que percebe-se um diagrama em formato retangular, porém com oscilações no feixe principal acima do especificado. Esse ripple é característico para pequenas aberturas (pequeno número de células). Verifica-se discrepâncias entre os resultados obtidos pela ferramenta e HFSS. Após realizada a correção nas amplitudes dos elementos com dimensões mais afastadas da dimensão de ressonância, tem-se os resultados apresentados nas Figuras 5.12 e 5.13. 


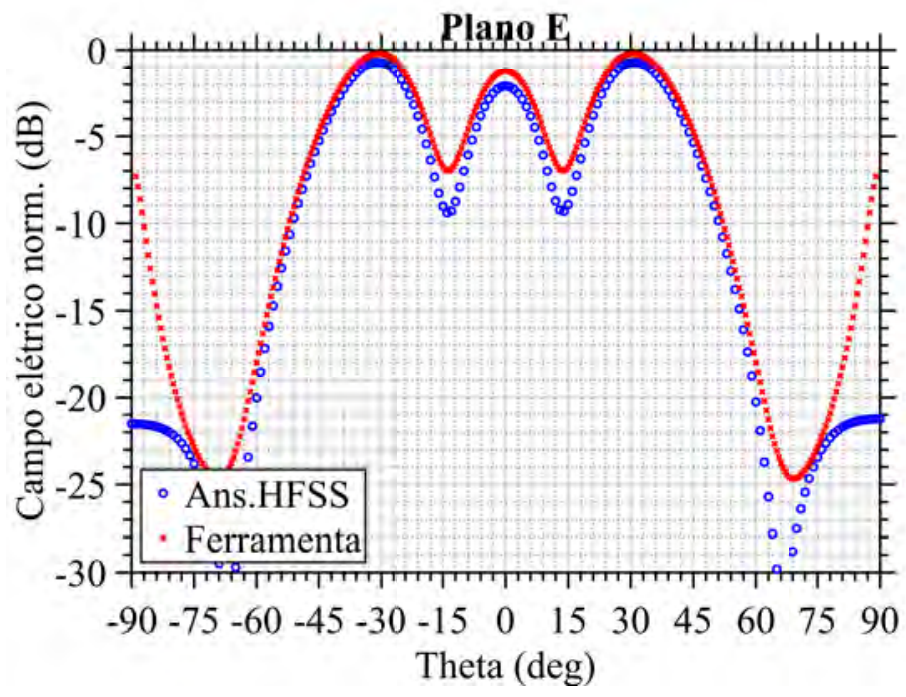

a)

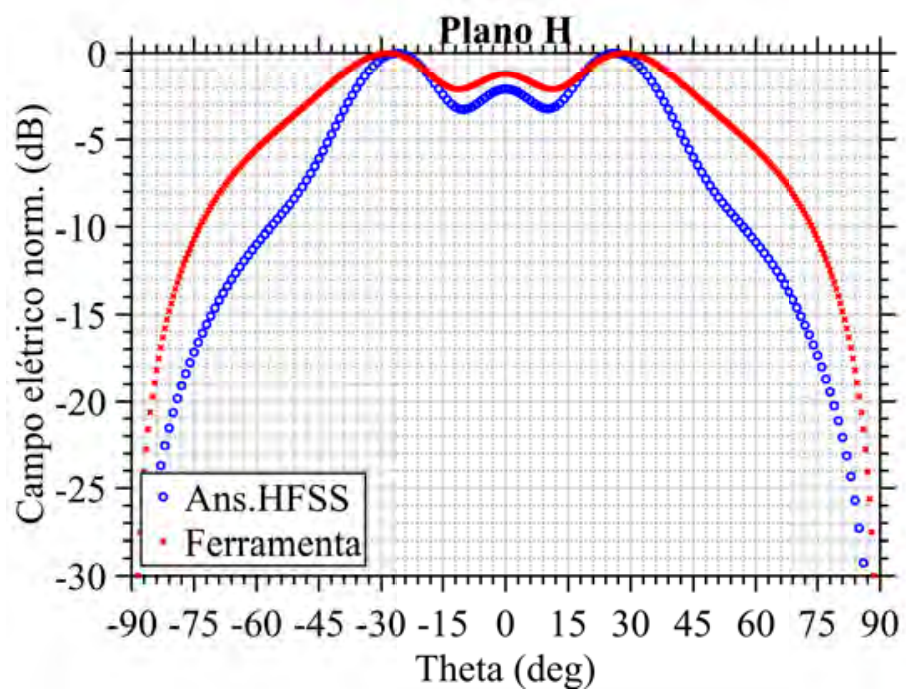

b)

Figura 5.10: Campo calculado e simulado para uma rede $5 \times 5$ - onda plana com incidência normal. a) Plano E: $E_{\theta}\left(\theta, \phi=0^{\circ}\right)$; b) Plano $\mathrm{H}: E_{\phi}\left(\theta, \phi=90^{\circ}\right)$. 


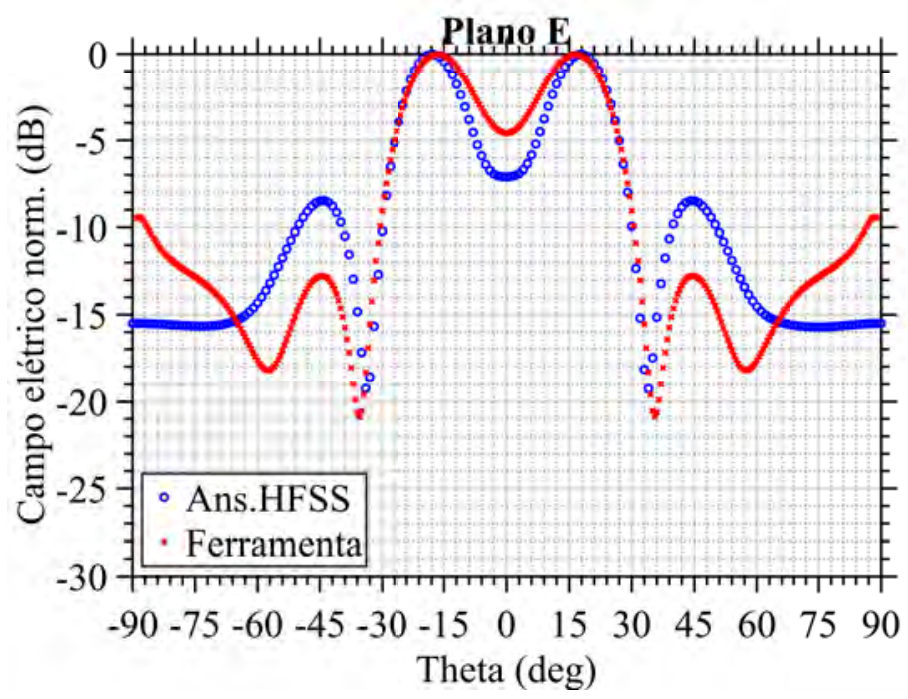

a)

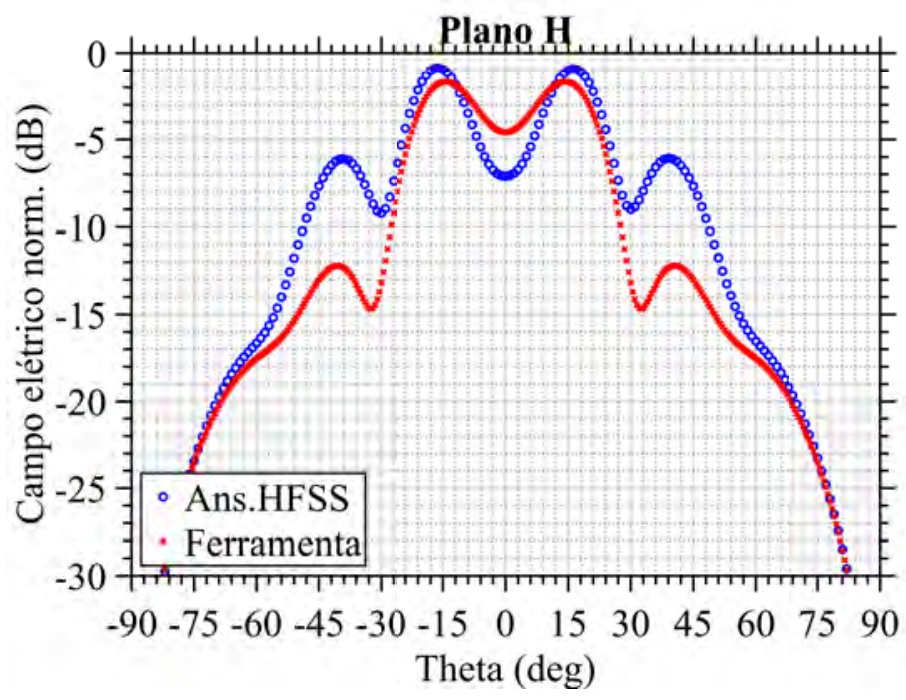

b)

Figura 5.11: Campo calculado e simulado para uma rede $7 \times 7$ - onda plana com incidência normal. a) Plano E: $E_{\theta}\left(\theta, \phi=0^{\circ}\right)$; b) Plano $\mathrm{H}: E_{\phi}\left(\theta, \phi=90^{\circ}\right)$. 


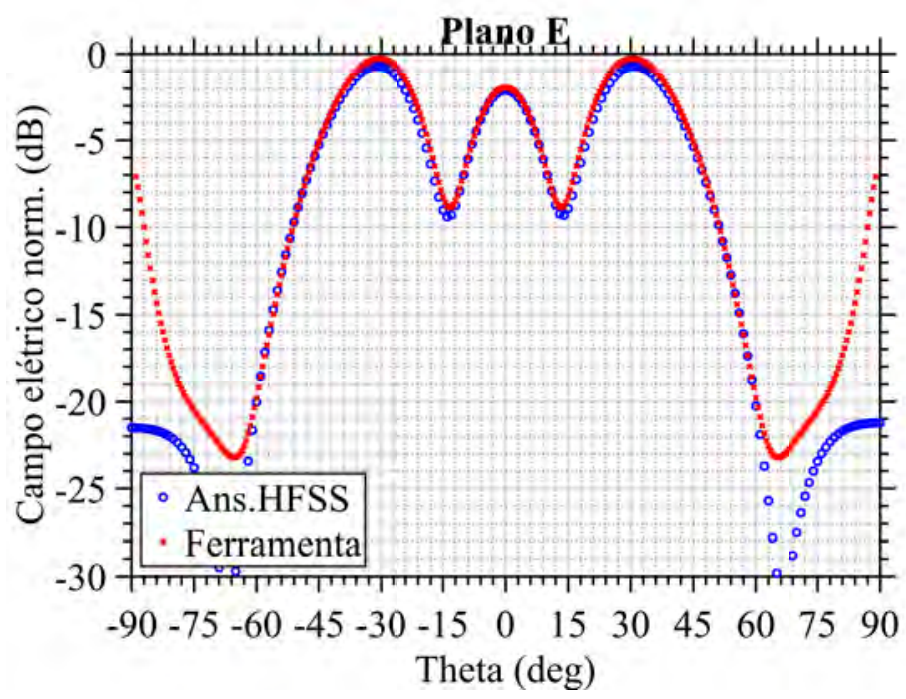

a)

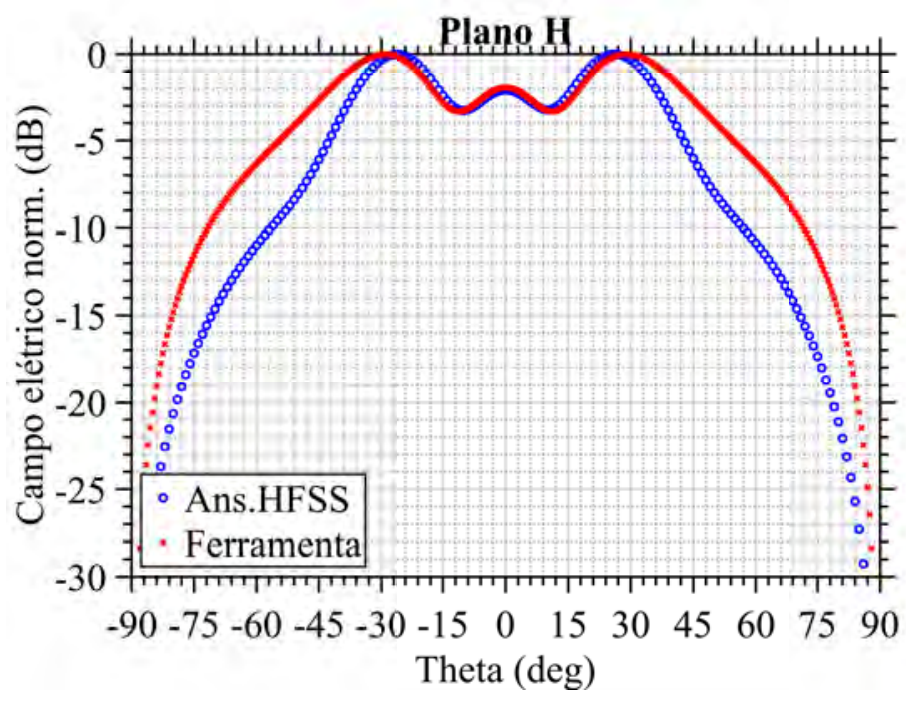

b)

Figura 5.12: Campo calculado e simulado para uma rede $5 \times 5$ após correções pontuais - onda plana com incidência normal. a) Plano E: $E_{\theta}\left(\theta, \phi=0^{\circ}\right)$; b) Plano $\mathrm{H}: E_{\phi}\left(\theta, \phi=90^{\circ}\right)$. 


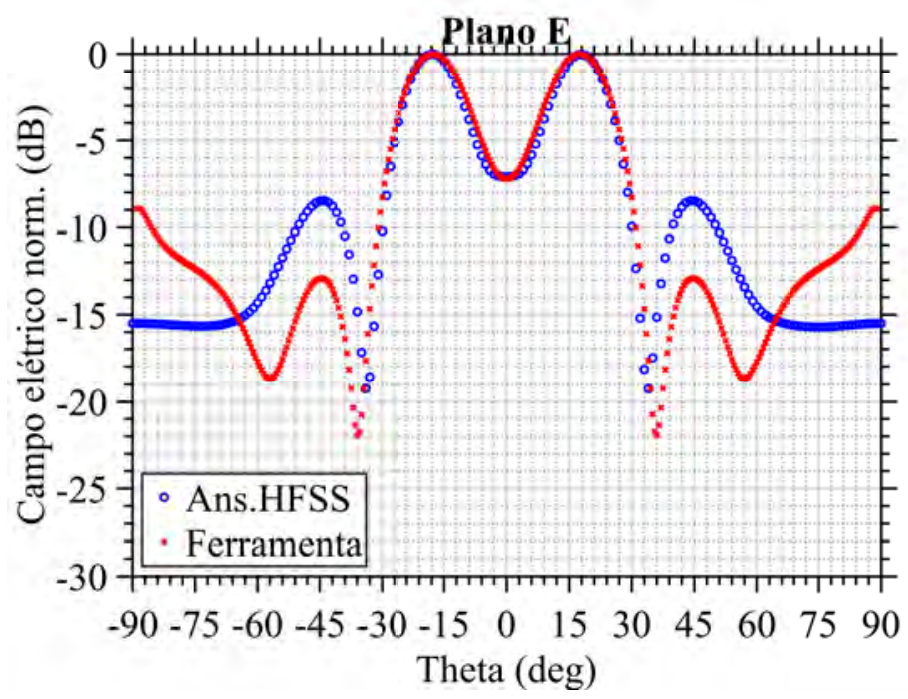

a)

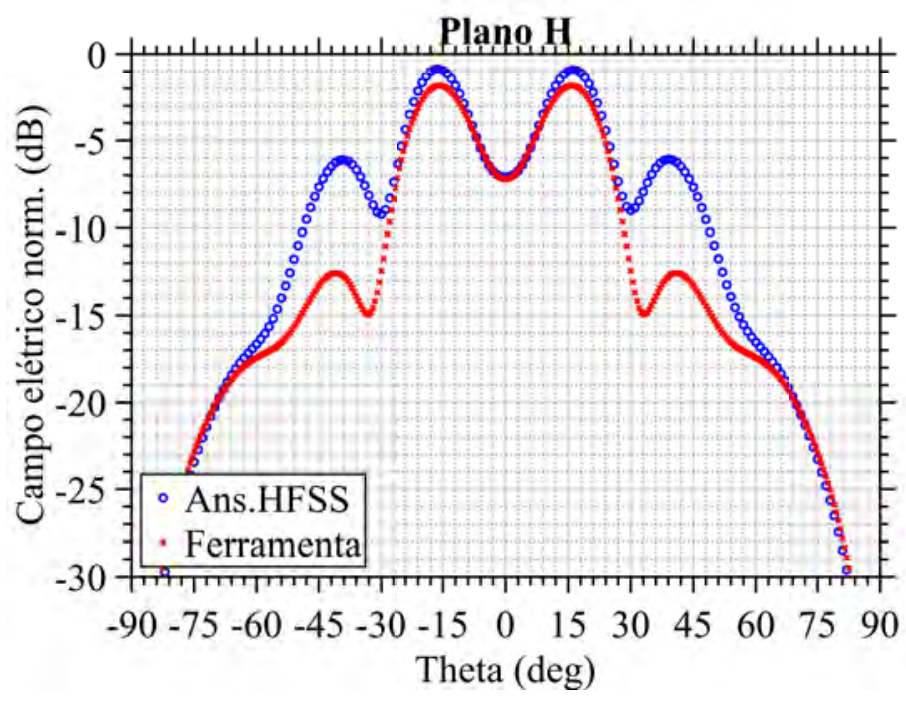

b)

Figura 5.13: Campo calculado e simulado para uma rede $7 \times 7$ após correções pontuais - onda plana com incidência normal. a) Plano E: $E_{\theta}\left(\theta, \phi=0^{\circ}\right)$; b) Plano H: $E_{\phi}\left(\theta, \phi=90^{\circ}\right)$.

Nas Figuras 5.14 e 5.15 são ilustrados o diagrama de irradiação 3D com contorno e a vista isométrica do campo elétrico tridimensional re-irradiado no software HFSS para as redes projetadas, respectivamente. 


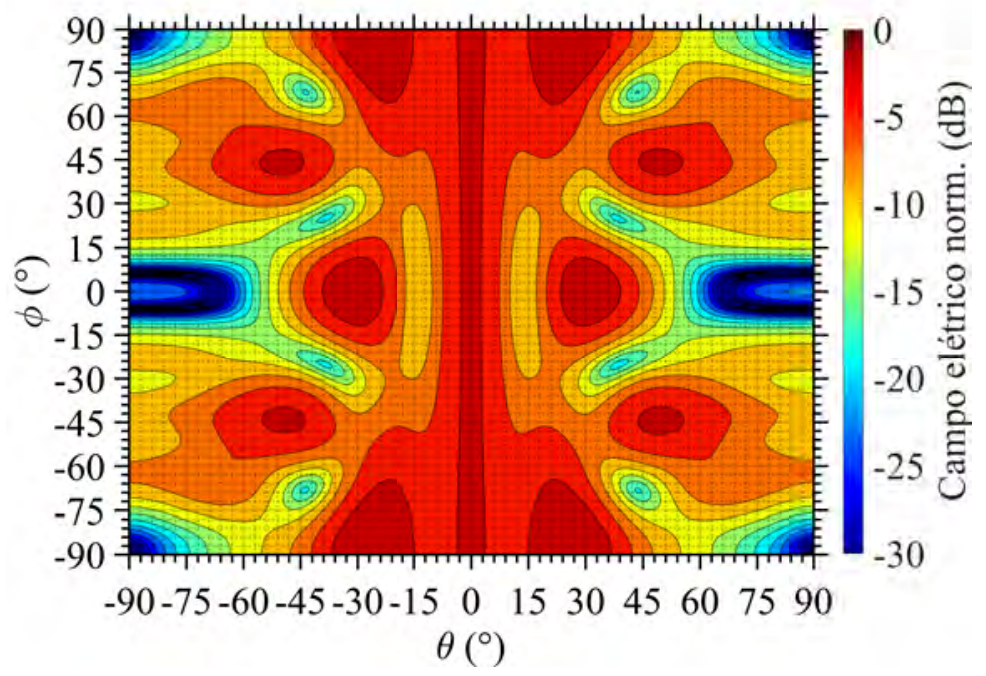

a)

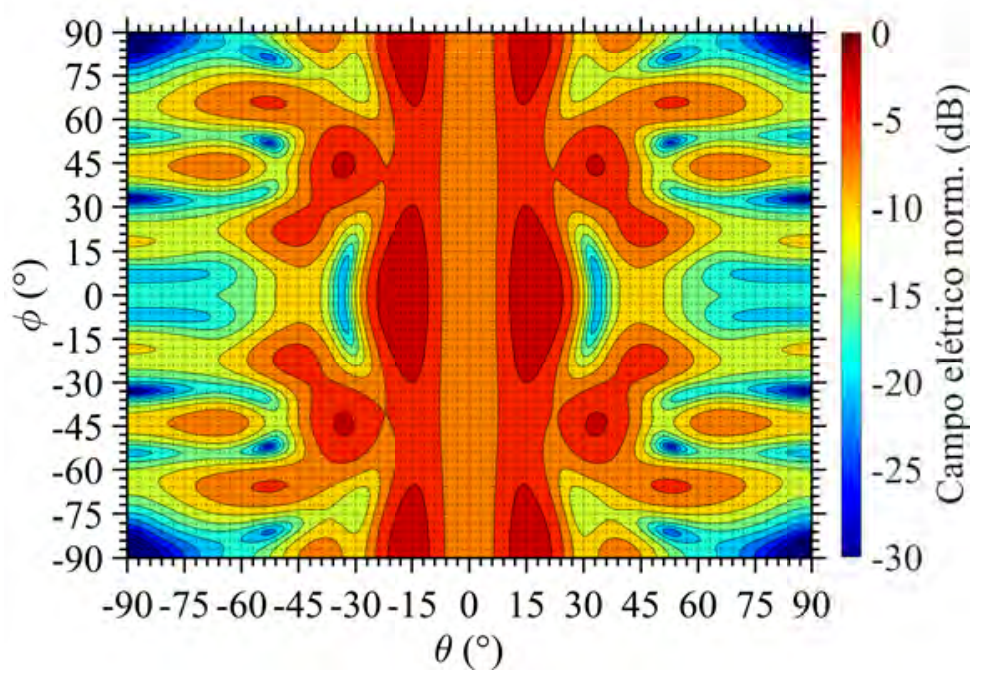

b)

Figura 5.14: Diagramas de re-irradiação com contorno. a) RA $5 \times 5$; b) RA $7 \times 7$.

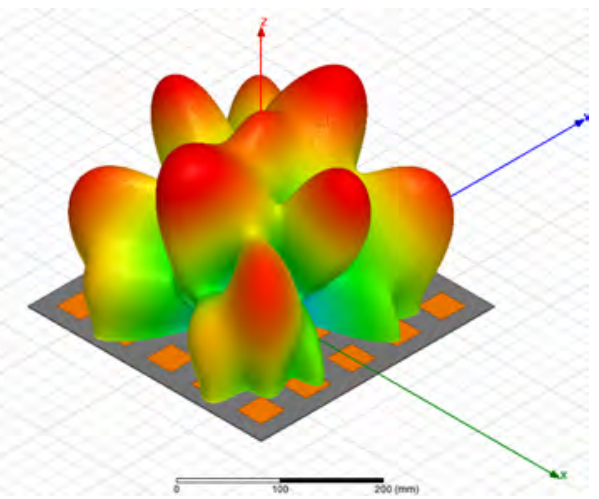

a)

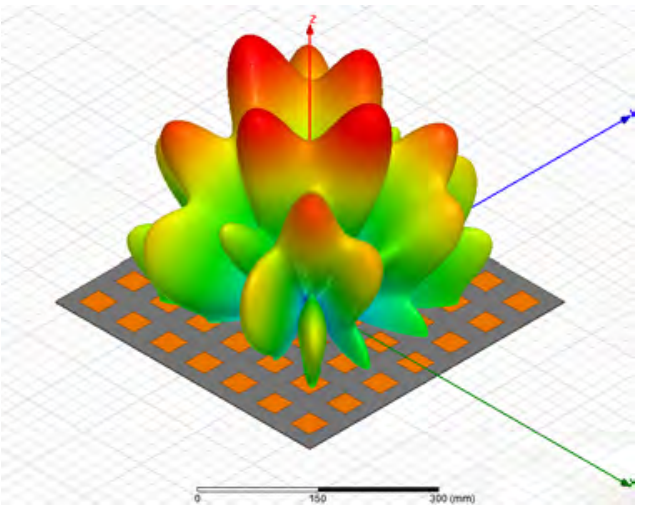

b)

Figura 5.15: Diagramas de re-irradiação 3D conformado. a) RA 5×5; b) RA $7 \times 7$. 


\section{6}

\section{Conclusão e trabalhos futuros}

Neste trabalho é apresentada a formulação da função diádica de Green para estruturas multicamada utilizando o método do circuito equivalente, que considera substratos e plano de terra infinitos. O método dos momentos é aplicado para se determinar numericamente as densidades de corrente superficial sobre os patches que compõem redes refletoras. Adicionalmente, funções base com condição de borda segmentada são deduzidas e utilizadas para modelar o comportamento impulsivo da densidade de corrente elétrica superficial na direção transversal do campo elétrico tangencial incidente nos elementos espalhadores.

A aplicação da segmentação permite a redução da memória computacional utilizada no preenchimento das matrizes do método dos momentos. As curvas de fase e amplitude, e os campos elétricos espalhados por elementos simples e por redes $3 \times 3,5 \times 5$ e $7 \times 7$, apresentam excelente concordância com o Ansys Designer, sendo o número de funções base no mínimo 100 vezes menor que o software comercial.

Inicialmente, o campo refletido no plano de terra/substrato foi acrescentado no cálculo do campo elétrico total re-irradiado pela estrutura. Percebe-se que a inclusão da reflexão possibilita o aumento da faixa de variação de fase quando o resultado é comparado com a curva de fase do campo espalhado isolado. Percebem-se discrepâncias entre os diagramas de irradiação no plano E para a região dos lóbulos secundários próximos de $90^{\circ}$, que tendem a ser minimizadas à medida que o número de elementos aumenta. Também, um deslocamento em frequência de $70 \mathrm{MHz}$ da curva de fase em relação ao software Ansys HFSS é constatado. Como alternativa para ambos os problemas, a formulação de uma função de Green que considera uma estrutura finita pode ser formulada em trabalhos futuros.

Além disso, constatam-se diferenças na amplitude dos lóbulos laterais nos diagramas de irradiação no plano H. Para minimizar o erro, a consideração dos efeitos de difração de borda foi analisada. O método das correntes equivalentes implementado encontra-se livre de singularidades comuns em regiões de transição (sombra do campo incidente e refletido), como percebido na teoria geométrica da difração. 
Adicionalmente, os efeitos difrativos foram avaliados sobre as curvas de fase das células. Verifica-se que o impacto é praticamente desprezível, visto que é mais significativo na região dos lóbulos laterais do plano H. Entretanto, a curva de fase é obtida a partir da avaliação do campo elétrico irradiado para a direção especular do plano E, e, neste caso, o campo difratado é pouco intenso.

Também, constatam-se diferenças na amplitude de alguns lóbulos laterais nos diagramas de irradiação sintetizados em decorrência da falta de acurácia na amplitude do campo elétrico calculado para elementos que possuem dimensões afastadas da ressonância. Tal motivo está relacionado ao comportamento da função base utilizada. Como forma de comprovação, modificou-se pontualmente em $2 \%$ a amplitude do campo elétrico re-irradiado por células específicas. Como trabalhos futuros, a melhoria da representação da função base pode ser investigada.

A modelagem eletromagnética aqui descrita pode ser aplicada no projeto de redes refletoras com poucos elementos, em que a análise de Floquet, que é normalmente aplicada por vários autores, não é viável. Desta forma, como trabalho futuro, uma rede refletora será projetada e construída para a validação experimental da técnica proposta.

Além disso, o projeto e inclusão de fontes de excitação mais realistas devem ser incorporadas no modelo matemático, a fim de tornar a análise ainda mais robusta e precisa. 


\section{Referências bibliográficas}

[1] HODGES, R. E.; CHAHAT, N.; HOPPE, D. J.; VACCHIONE, J. D.. A deployable high-gain antenna bound for mars: Developing a new folded-panel reflectarray for the first cubesat mission to mars. IEEE Antennas and Propagation Magazine, 59:39- 49, 2017.

[2] BALANIS, C. A.. Antenna Theory: Analysis and Design. Wiley, New Jersey, 3rd edition, 2005.

[3] ENCINAR,J. A.; FLORENCIO, R.; ARREBOLA, M.; SALAS, M. A.; BARBA, M.; BOIX, R. R.; TOSO, G.. Dual-polarization reflectarray in ku-band based on two layers of dipole-arrays for a transmitreceive satellite antenna with south american coverage. In: 11TH EUROPEAN CONFERENCE ON ANTENNAS AND PROPAGATION (EUCAP), p. 86-89, Paris, França, 2017.

[4] BERGMANN, J. R.; MOREIRA, F. J. S.. Omnidirectional dualreflector antenna with go shaped main reflector for pattern control in the elevation plane: Oadc case. International Journal of Antennas and Propagation, 2012:1-6, 2012.

[5] FLORENCIO, R.; BOIX, R. R.; ENCINAR, J. A.. Enhanced integral equation analysis of multilayered periodic structures useful for the design of reflectarray antennas. In: INTERNATIONAL CONFERENCE ON ELECTROMAGNETICS IN ADVANCED APPLICATIONS (ICEAA), p. 86-89, Turin, Itália, 2015.

[6] HUANG, J.; ENCINAR, J. A.. Reflectarray antennas. IEEE Press/Wiley, New York, 1st edition, 2008.

[7] YANG, H.; CHEN, X.; YANG, F.; XU, S.; CAO, X.; LI, M.; GAO, J.. Design of resistor-loaded reflectarray elements for both amplitude and phase control. IEEE Antennas and Wireless Propagation Letters, 16:1159-1162, 2017.

[8] KARIMIPOUR, M.; PIRHADI, A.. A novel approach to synthesis of non-uniform conformal reflectarray antennas. The Applied 
Computational Electromagnetics Society (ACES JOURNAL), 28:1040-1049, 2013.

[9] DERAFSHI, I.; KOMJANI, N.; MOHAMMADIRAD, M.. A single-layer broadband reflectarray antenna by using quasi-spiral phase delay line. IEEE Antennas and Wireless Propagation Letters, 14:84-87, 2015.

[10] NGUYEN, B. D.; PHAM, K. T.; TRAN, V.; MAI, L.; YONEMOTO, N.. Reflectarray element using cut-ring patch coupled to delay line. IEEE Antennas and Wireless Propagation Letters, 14:571-574, 2015.

[11] MALFAJANI, R. S.; ATLASBAF, Z.. Design and implementation of a broadband single layer circularly polarized reflectarray antenna. IEEE Antennas and Wireless Propagation Letters, 11:973-976, 2012.

[12] MALFAJANI, R. S.; ATLASBAF, Z.. Design and implementation of a dual-band single layer reflectarray in $\mathrm{x}$ and $\mathrm{k}$ bands. IEEE Transactions on Antennas and Propagation, 62:4425 - 4431, 2014.

[13] HAN, C.; ZHANG, Y.; YANG, Q.. A broadband reflectarray antenna using triple gapped rings with attached phase-delay lines. IEEE Transactions on Antennas and Propagation, 65:2713 - 2717, 2017.

[14] ETHIER, J.; MCNAMARA, D.A.; CHAHARMIR, M.R.; SHAKER, J.. Aperture-coupled reflectarray element with wide range of phase delay. Electronics Letters, 48:900-902, 2012.

[15] ETHIER, J.; MCNAMARA, D.A.; CHAHARMIR, M.R.; SHAKER, J.. Closer look at mosaic reflectarray antennas. Electronics Letters, 51:437-438, 2015.

[16] SAYIDMARIE, K.H.; BIALKOWSKI, M.E.. Fractal unit cells of increased phasing range and low slopes for single-layer microstrip reflectarrays. IET Microwaves, Antennas e Propagation, 5:1371-1379, 2011.

[17] OlOUMI, D.; EBADI, S.; KORDZADEH, A.; ET AL.. Miniaturized reflectarray unit cell using fractal-shaped patch slot configuration. IEEE Antennas and Wireless Propagation Letters, 11:10-13, 2012.

[18] COSTANZO, S.; VENNERI, F.. Miniaturized fractal reflectarray element using fixed-size patch. IEEE Antennas and Wireless Propagation Letters, 13:1437-1440, 2014. 
[19] MOUSTAFA, L.; GILLARD, R.; PERIS, F.; LOISON, R.; LEgAY, H.; GIRARD, E.. The phoenix cell: A new reflectarray cell with large bandwidth and rebirth capabilities. IEEE Antennas and Wireless Propagation Letters, 10:71-74, 2011.

[20] DENG, R.; YANG, F.; XU, S.; LI, M.. A low-cost metal-only reflectarray using modified slot-type phoenix element with $360^{\circ}$ phase coverage. IEEE Transactions on Antennas and Propagation, 64:1556 1560, 2016.

[21] DENG, R.; XU, S; YANG, F.; LI, M.. Design of a low-cost single-layer $\mathrm{x} / \mathrm{ku}$ dual-band metal-only reflectarray antenna. IEEE Antennas and Wireless Propagation Letters, 16:2106-2109, 2017.

[22] ETHIER, J.; CHAHARMIR, M. R.; SHAKER, J.. Loss reduction in reflectarray designs using sub-wavelength coupled-resonant elements. IEEE Transactions on Antennas and Propagation, 60:5456 - 5459, 2012.

[23] ETHIER, J.; CHAHARMIR, M. R.; SHAKER, J.; LEE, D.. Development of novel low-cost reflectarray. IEEE Antennas and Propagation Magazine, 54:277 - 287, 2012.

[24] VOSOOGH, A.; KEYGHOBAD, K.; KHALEGHI, A.; MANSOURI, S.. A high-efficiency ku-band reflectarray antenna using single-layer multiresonance elements. IEEE Antennas and Wireless Propagation Letters, 13:891-894, 2014.

[25] XUE, F.; WANG, H.; YI, M.; LIU, G.; DONG, X.. Design of a broadband single-layer linearly polarized reflectarray using four-arm spiral elements. IEEE Transactions on Antennas and Propagation, 16:696-699, 2017.

[26] AN, W.; XU, S.; YANG, F.. A metal-only reflectarray antenna using slot-type elements. IEEE Antennas and Wireless Propagation Letters, 13:1553-1556, 2014.

[27] QU, S.; CHEN, Q.; XIA M.; ZHANG, X. Y.. Single-layer dual-band reflectarray with single linear polarization. IEEE Transactions on Antennas and Propagation, 62:199 - 205, 2014.

[28] MONTERO, J. S.; MARTINEZ-LOPEZ, J. I.; RODRIGUEZ-CUEVAS, J.; MARTYNYUK, A. E.. Spiraphase-type reflectarray for large reflec- 
tion elevation angles. IEEE Transactions on Antennas and Propagation, 63:4342 - 4351, 2015.

[29] WU, G.; QU, S. M. C. Y. S. C. C.. Reflectarray antenna design with arbitrary incident and reflection beam angle. IEEE Transactions on Antennas and Propagation, 66:5964 - 5973, 2018.

[30] LEE, S. R.; LIM, E. H.; LO, F. L.; NG, W. H.. Circularly polarized elliptical microstrip patch reflectarray. IEEE Transactions on Antennas and Propagation, 65:4322 - 4327, 2017.

[31] FLORENCIO, R.; ENCINAR, J. A.; BOIX, R. R.; LOSADA, V.; TOSO, G.. Reflectarray antennas for dual polarization and broadband telecom satellite applications. IEEE Transactions on Antennas and Propagation, 63:1234 - 1246, 2015.

[32] YOON, J. H.; YOON, Y. J.; LEE, W.; SO, J.. Broadband microstrip reflectarray with five parallel dipole elements. IEEE Antennas and Wireless Propagation Letters, 14:1109-1112, 2015.

[33] ZHANG, L.; GAO, S.; LUO, Q.; LI, W.; HE, Y.; LI, Q.. Single-layer wideband circularly polarized high-efficiency reflectarray for satellite communications. IEEE Transactions on Antennas and Propagation, 65:4529-4538, 2017.

[34] ZHAO, M.; ZHANG, G.; LEI, X.; WU, J.; SHANG, J.. Design of new single-layer multiple-resonance broadband circularly polarized reflectarrays. IEEE Antennas and Wireless Propagation Letters, 12:356-359, 2013.

[35] SMITH, T.; GOTHELF, U.; KIM, O. S.; BREINBJERG, O.. Design, manufacturing, and testing of a 20/30-ghz dual-band circularly polarized reflectarray antenna. IEEE Antennas and Wireless Propagation Letters, 12:1480-1483, 2013.

[36] FUENTES, J. A. O.; MONTERO, J. S.; LOPEZ, J. I. M.; CUEVAS, J. R.; MARTYNYUK, A. E.. Dual-frequency reflectarray based on splitring slots. IEEE Antennas and Wireless Propagation Letters, 16:952-955, 2017.

[37] YU, J.; WANG, J.; CHEN, L.; YANG, J. Y.; SHI, X.. Design of circularly polarised beam-shaped ras using a single layer of concentric dual split loops. IET Microwaves, Antennas e Propagation, 10:1515-1521, 2016. 
[38] YU, A.; YANG, F.; ELSHERBENI, A. Z; HUANG, J.; KIM, Y.. An offset-fed $\mathrm{x}$-band reflectarray antenna using a modified element rotation technique. IEEE Transactions on Antennas and Propagation, 60:1619 - 1624, 2012.

[39] DENG, R.; MAO, Y.; XU, S.; YANG, F.. A single-layer dual-band circularly polarized reflectarray with high aperture efficiency. IEEE Transactions on Antennas and Propagation, 63:3317 - 3320, 2015.

[40] YANG, X.; XU, S.; YANG, F.; LI, M.; HOU, Y.; JIANG, S.; LIU, L.. A broadband high-efficiency reconfigurable reflectarray antenna using mechanically rotational elements. IEEE Transactions on Antennas and Propagation, 65:3959 - 3966, 2017.

[41] ZAMUDIO, J. R.; LOPEZ, J. I. M.; CUEVAS, J. R.; MARTYNYUK, A. E.. Reconfigurable reflectarrays based on optimized spiraphasetype elements. IEEE Transactions on Antennas and Propagation, 60:1821 $-1830,2012$.

[42] NGUYEN, D. B.; PHAM, K. T.; TRAN, V.; MAI, L.; YONEMOTO, N.; KOHMURA, A.; FUTATSUMORI, S.. Electronically tunable reflectarray element based on c-patch coupled to delay line. Electronics Letters, 50:1114-1116, 2014.

[43] WANG, Y.; TENNANT, A.. Experimental time-modulated reflector array. IEEE Transactions on Antennas and Propagation, 62:6533 - 6536, 2014.

[44] LI, Y.; ABBOSH, A.. Reconfigurable reflectarray antenna using single-layer radiator controlled by pin diodes. IET Microwaves, Antennas e Propagation, 9:664-671, 2015.

[45] ZHANG, M. T.; GAO, S.; JIAO, Y.; WAN, J. X.; TIAN, B. N.; WU, C. B.; FARRALL, A. J.. Design of novel reconfigurable reflectarrays with single-bit phase resolution for $\mathrm{ku}$-band satellite antenna applications. IEEE Transactions on Antennas and Propagation, 64:1634 - 1641, 2016.

[46] YANG, H.; YANG, F.; XU, S.; MAO, Y.; LI, M.; CAO, X.; GAO, J.. A 1-bit $10 \times 10$ reconfigurable reflectarray antenna: Design, optimization, and experiment. IEEE Transactions on Antennas and Propagation, 64:2246 - 2254, 2016. 
[47] YANG, H.; YANG, F.; CAO, X.; XU, S.; GAO, J.; CHEN, X.; LI, M.; LI, T.. A 1600-element dual-frequency electronically reconfigurable reflectarray at $\mathrm{x} / \mathrm{ku}$-band. IEEE Transactions on Antennas and Propagation, 65:3024 - 3032, 2017.

[48] LI, Y.; ABBOSH, A.. Electronically controlled phasing element for single-layer reconfigurable reflectarray. IEEE Antennas and Wireless Propagation Letters, 11:628-631, 2012.

[49] CARRASCO, E.; BARBA, M.; REIG, B.; DIEPPEDAlE, C.; ENCINAR, J. A.. Characterization of a reflectarray gathered element with electronic control using ohmic rf mems and patches aperturecoupled to a delay line. IEEE Transactions on Antennas and Propagation, 60:4190 - 4201, 2012.

[50] DEBOGOVIC, T.; CARRIER, J. P.. Low loss mems-reconfigurable 1-bit reflectarray cell with dual-linear polarization. IEEE Transactions on Antennas and Propagation, 62:5055 - 5060, 2014.

[51] PEREZ-PALOMINO, G.; FLORENCIO, R.; ENCINAR, J. A.; BARBA, M.; DICKIE, R.; CAHILL, R.; BAINE, P.; BAIN, M.; BOIX, R. R.. Accurate and efficient modeling to calculate the voltage dependence of liquid crystal-based reflectarray cells. IEEE Transactions on Antennas and Propagation, 62:2659-2668, 2014.

[52] NAYERI, P.; YANG, F.; ELSHERBENI, A. Z.. Beam-scanning reflectarray antennas: A technical overview and state of the art. IEEE Antennas and Propagation Magazine, 57:32 - 47, 2015.

[53] ELHADY, M. A.; HONG, W.; ZHANG, Y.. A ka-band reflectarray implemented with a single-layer perforated dielectric substrate. IEEE Antennas and Wireless Propagation Letters, 11:600-603, 2012.

[54] TAHER, M. K.; NUAIMI, A.; HONG, W.. Discrete dielectric reflectarray and lens for e-band with different feed. IEEE Antennas and Wireless Propagation Letters, 13:947-950, 2014.

[55] BOOKET, M. R.; ATLASBAF, Z.; SHAHABADI, M.. Broadband reflectarray antenna on a periodically perforated substrate. IEEE Transactions on Antennas and Propagation, 64:3711-3717, 2016.

[56] NAYERI, P.; LIANG, M.;GARCÍA, R. A. S.; TUO, M.; YANG, F.; GEHM, M.; XIN, H.; ELSHERBENI, A. Z.. 3d printed dielectric reflectarrays: 
Low-cost high-gain antennas at sub-millimeter waves. IEEE Transactions on Antennas and Propagation, 62:2000-2008, 2014.

[57] REN, J.; MENZEL, W.. Dual-frequency folded reflectarray antenna. IEEE Antennas and Wireless Propagation Letters, 12:1216-1219, 2013.

[58] REN, J.; MENZEL, W.. A folded reflectarray antenna with a planar siw slot array antenna as the primary source. IEEE Transactions on Antennas and Propagation, 62:3575-3583, 2014.

[59] LUO, Q.; GAO, S.; ZHANG, C.; ZHOU, D.; CHALOUN, T.; MENZEL, W.; ZIEGLER, V.; SOBHY, M.. Design and analysis of a reflectarray using slot antenna elements for ka-band satcom. IEEE Transactions on Antennas and Propagation, 63:1365-1374, 2015.

[60] QU, S. W.; ZHANG, H. X.; WU, W. W.; LI, P. F.; YANG, S.; NIE, Z. P.. Wideband folded reflectarray using novel elements with high orthogonal polarization isolation. IEEE Transactions on Antennas and Propagation, 64:3195-3200, 2016.

[61] LEI, X. Y.; CHENG, Y. J.. High-efficiency and high-polarization separation reflectarray element for oam-folded antenna application. IEEE Antennas and Wireless Propagation Letters, 16:1357-1360, 2017.

[62] ZHOU, M. Accurate Modeling of Advanced Reflectarrays. Tese de doutorado em engenharia elétrica, Technical University of Denmark, Kongens Lyngby, 2012.

[63] MOHARRAM, M. A.; KISHK, A. A.. Optimum feeds for reflectarray antenna: Synthesis and design. IEEE Transactions on Antennas and Propagation, 64:469-483, 2016.

[64] ARREBOlA, M.; AlVAREZ, Y.; ENCINAR, J. A.; LAS-HERAS, F.. Accurate analysis of printed reflectarrays considering the near field of the primary feed. IET Microwaves, Antennas e Propagation, 3:187194, 2009.

[65] LAURIN, J. J.; EL HANI, R.. Specular reflection analysis for offspecular reflectarray antennas. IEEE Transactions on Antennas and Propagation, 61:3575-3581, 2013.

[66] MOHAMMADIRAD, M.; KOMJANI, N.; CHAHARMIR, M. R.; SHAKER, J.; SEBAK, A. R.. Impact of feed position on the operating band 
of broadband reflectarray antenna. IEEE Antennas and Wireless Propagation Letters, 11:1104-1107, 2012.

[67] ALMAJALI, E.; MCNAMARA, D. A.; SHAKER, J.; CHAHARMIR, M. R.. On beam squint in offset-fed reflectarrays. IEEE Antennas and Wireless Propagation Letters, 11:937-940, 2012.

[68] ALMAJALI, E.; MCNAMARA, D. A.; SHAKER, J.; CHAHARMIR, M. R.. Beam squint suppression in offset-fed reflectarrays. IEEE Antennas and Wireless Propagation Letters, 12:587-590, 2013.

[69] ALMAJALI, E.; MCNAMARA, D. A.; SHAKER, J.; CHAHARMIR, M. R.. Grating lobe control in dual-feed single-beam reflectarrays and power combiners. IEEE Antennas and Wireless Propagation Letters, 12:1268-1291, 2013.

[70] RIOJA, E. M.; ENCINAR, J. A.; BARBA, M.; FLORENCIO, R.; BOIX, R. R.; LOSADA, V.. Dual polarized reflectarray transmit antenna for operation in ku- and ka-bands with independent feeds. IEEE Transactions on Antennas and Propagation, 65:3241-3246, 2017.

[71] NAYERI, P.; ELSHERBENI, A. Z; YANG, F.. Radiation analysis approaches for reflectarray antennas. IEEE Antennas and Propagation Magazine, 55:127-134, 2013.

[72] POZAR,D. M.; TARGONSKI, S. D.; SYRIGOS, H. D.. Design of millimeter wave microstrip reflectarrays. IEEE Transactions on Antennas and Propagation, 45:287-296, 1997.

[73] DREHER, A.. A new approach to dyadic green's function in spectral domain. IEEE Transactions on Antennas and Propagation, 43:1297-1302, 1995.

[74] ALMAJALI, E.; MCNAMARA, D.; SHAKER, J.; CHAHARMIR, M. R.. Derivation and validation of the basic design equations for symmetric sub-reflectarrays. IEEE Transactions on Antennas and Propagation, 60:2336-2346, 2012.

[75] ROBUSTILlO, P.; ZAPATA, J.; ENCINAR, J. A.; RUBIO, J.. Ann characterization of multi-layer reflectarray elements for contouredbeam space antennas in the ku-band. IEEE Transactions on Antennas and Propagation, 60:3205-3214, 2012. 
[76] ZHOU, M.; SØRENSEN, S. B.; KIM, O. S.; JØRGENSEN, E.; MEINCKE, P.; BREINBJERG, O. ; TOSO, G.. The generalized direct optimization technique for printed reflectarrays. IEEE Transactions on Antennas and Propagation, 62(4):1690-1700, 2014.

[77] MILON, M. A.; CADORET, D.; GILLARD, R.; LEGAY, H.. Surroundedelement approach for the simulation of reflectarray radiating cells. IET Microwaves, Antennas e Propagation, 1:289-293, 2007.

[78] ALMAJALI, E.; MCNAMARA, D. A.; SHAKER, J. ; CHAHARMIR, M. R.. Feed image lobes in offset-fed reflectarrays: Diagnosis and solution. IEEE Transactions on Antennas and Propagation, 62(1):216227, 2014.

[79] KARIMIPOUR, M.; PIRHADI, A. ; EBRAHIMI, N.. Accurate method for synthesis of shaped-beam non-uniform reflectarray antenna. IET Microwaves, Antennas Propagation, 7(15):1247-1253, 2013.

[80] PRADO, D. R.; ARREBOLA, M.; PINO, M. R. ; LAS-HERAS, F.. Complex reflection coefficient synthesis applied to dual-polarized reflectarrays with cross-polar requirements. IEEE Transactions on Antennas and Propagation, 63(9):3897-3907, Sept 2015.

[81] POZAR, D.. Microwave Engineering. Wiley, New Jersey, 3rd edition, 2005.

[82] HARRINGTON, R. F.. Time harmonic electromagnetic fields. Wiley, New York, 1st edition, 2001.

[83] PRALON, M. G.. Aplicação do Método dos Momentos na Análise de Antenas Microfita. Dissertação de mestrado em engenharia elétrica, Pontifícia Universidade Católica do Rio de Janeiro - PUC-Rio, Rio de Janeiro, Brasil, 2012.

[84] GARG, R.; BHARTIA, P.; BAHL, I.; ITTIPIBOON, A.. Microstrip Antenna Design Handbook. Artech House, Norwood, 1st edition, 2001.

[85] RENGARAJAN, S. R.. Choice of basis functions for accurate characterization of infinite array of microstrip reflectarray elements. IEEE Antennas and Wireless Propagation Letters, 4:47-50, 2005.

[86] ZHOU, M.; JØRGENSEN, E.; KIM, O. S. ; SØRENSEN, S. B.; MEINCKE, P.; BREINBJERG, O.. Accurate and efficient analysis of printed 
reflectarrays with arbitrary elements using higher-order hierarchical legendre basis functions. IEEE Antennas and Wireless Propagation Letters, 11:814-817, 2012.

[87] ITOH, T. Numerical techniques for microwave and millimeterwave passive structures. Wiley-Interscience, New York, USA, 1st edition, 1989.

[88] RIBEIRO, R. O. ; HECKLER, M. V. T. . T.-S. A. F.. Entire domain basis function with accurate edge condition for rectangular microstrip antennas. IEEE Antennas and Wireless Propagation Letters, 18(1):123 - 127, 2019.

[89] POZAR, D.. Input impedance and mutual coupling of rectangular microstrip antennas. IEEE Transactions on Antennas and Propagation, 30:1191 - 1196, 1982.

[90] DATTHANASOMBAT, S.. Analysis and design of high-gain spacefed passive microstrip array antennas. Tese de doutorado em engenharia elétrica, University of Southern California, Los Angeles, California, 2003.

[91] CHEW, W. C.. Waves and Fields in Inhomogenous Media. WileyIEEE Press, New York, 1st edition, 1999.

[92] KELLER, J. B.. Geometrical theory of diffraction. Journal of Optical Society of America, 52:116-130, 1962.

[93] KOUYOUMJIAN, R. G.; PATHAK, P. H.. A uniform geometrical theory of diffraction for an edge in a perfectly conducting surface. Proceedings of IEEE, 62:1448-1461, 1974.

[94] CLEMMOV, P. C.. Edge currents in diffraction theory. IEEE Transactions on Antennas and Propagation, 4:282-287, 1956.

[95] MILLAR, R. F.. An approximate theory of diffraction of an electromagnetic wave by an aperture in a plane screen. Proc. Inst. Elec. Eng., 103:177-185, 1956.

[96] RYAN, C. E.; PETERS, L.. Evaluation of edge-diffracted fields including equivalent currents for the caustic regions. IEEE Transactions on Antennas and Propagation, 17:292-299, 1960. 
[97] UFIMTSEV, P. Y.. Elementary waves and the physical theory of diffraction. translation from Russian prepared by the U.S. Air Force Foreign Technology Division, Wright-Patterson AFB, Ohio, 1971.

[98] UFIMTSEV, P. Y.. Method of edge waves in the physical theory of diffraction. Electromagnetics, 11:125-160, 1991.

[99] MICHAELI, A.. Equivalent edge currents for arbitrary aspects of observation. IEEE Transactions on Antennas and Propagation, 32:252258, 1984.

[100] MICHAELI, A.. Correction to equivalent edge currents for arbitrary aspects of observation. IEEE Transactions on Antennas and Propagation, 33:227-227, 1985.

[101] MICHAELI, A.. Elimination of infinities in equivalent edge currents, part i fringe current components. IEEE Transactions on Antennas and Propagation, 34:912-918, 1986.

[102] MICHAELI, A.. Elimination of infinities in equivalent edge currents, part ii physical optics components. IEEE Transactions on Antennas and Propagation, 34:1034-1037, 1986.

[103] REGO, C. G.. Formulações assintóticas para o espalhamento por superfícies condutoras no domínio do tempo e aplicações à análise de transientes em antenas refetoras. Tese de doutorado em engenharia elétrica, Pontifícia Universidade Católica do Rio de Janeiro PUC-Rio, Rio de Janeiro, Brasil, 2001.

[104] APAYDIN, G.; HACIVELIOGLU, F. S. L. G. W. B. U.-P. Y.. Diffraction at a rectangular plate: First-order ptd approximation. IEEE Transactions on Antennas and Propagation, 64:1891-1899, 2016.

[105] ZHOU, M.; SØRENSEN, S. B.; JØRGENSEN, E.; MEINCKE, P.; KIM, O. S.; BREINBJERG, O.. An accurate technique for calculation of radiation from printed reflectarrays. IEEE Antennas and Wireless Propagation Letters, 10:1081 - 1084, 2011.

[106] HAJIAN, M.. Passive and active reconfigurable microstrip reflectarray antennas. PhD thesis, Delft University of Technology, Holanda-NL, 2008.

[107] FARIAS, R. L.; PEIXEIRO, C. H. M. V. T.. Circularly polarized $2 \times 2$ microstrip antenna array for application as a reflectarray feed. Microw Opt. Technol. Lett., 1:1-7, 2018. 
[108] SCHLOSSER, E. R.; TOLFO, S. M.; HECKLER, M. V. T.. Particle swarm optimization for antenna arrays synthesis. In: SBMO/IEEE MTTS INTERNATIONAL MICROWAVE AND OPTOELECTRONICS CONFERENCE (IMOC), p. 1-6, Porto de Galinhas, Brazil, 2015.

[109] YOSHIMOTO, E; SCHLOSSER, E. R. H. M. V. T.. Optimisation of antenna arrays installed on non-conductive unmanned aerial vehicle. IET Microwaves, Antennas and Propagation, 12:2292-2300, 2018. 
A

Formulação da função diádica de Green desnormalizada

A função diádica de Green foi obtida considerando a normalização das dimensões espaciais e, consequentemente, nas constantes de propagação. Entretanto, essas considerações podem ser removidas através das seguintes relações,

$$
\xi=\frac{\bar{\xi}}{k_{0}}
$$

$\mathrm{e}$

$$
k_{\xi}=k_{\bar{\xi}} k_{0}
$$

sendo $\xi=x, y$ ou $z$. Assim, as expressões são reescritas como sendo,

$$
\overline{\tilde{Y}}_{0}=-\frac{1}{k_{0} k_{z_{0}}} \overline{\tilde{y}}_{0}
$$

$\mathrm{e}$

$$
\overline{\tilde{Y}}_{n+1}=-\frac{1}{k_{0} k_{z_{n+1}}} \overline{\tilde{y}}_{n+1}
$$

com

$$
\overline{\tilde{y}}_{i}=\left[\begin{array}{cc}
\varepsilon_{r_{i}} k_{0}^{2}-k_{x}^{2} & k_{x} k_{y} \\
k_{x} k_{y} & \varepsilon_{r_{i}} k_{0}^{2}-k_{y}^{2}
\end{array}\right]
$$

$\mathrm{e}$

$$
\begin{gathered}
\overline{\tilde{V}}_{i}=\overline{\tilde{B}}_{i}=\left[\begin{array}{cc}
\cosh \left(k_{z_{i}} d_{i}\right) & 0 \\
0 & \cosh \left(k_{z_{i}} d_{i}\right)
\end{array}\right], \\
\overline{\tilde{Z}}_{i}=\frac{1}{\alpha_{i} \varepsilon_{r_{i}} k_{z_{i}}^{2}}\left[\begin{array}{cc}
\left(k_{y}^{2}-\varepsilon_{r_{i}} k_{0}^{2}\right) & k_{x} k_{y} \\
k_{x} k_{y} & \left(k_{x}^{2}-\varepsilon_{r_{i}} k_{0}^{2}\right)
\end{array}\right]
\end{gathered}
$$

$\mathrm{e}$

$$
\overline{\tilde{Y}}_{i}=\frac{1}{\alpha_{i} k_{z_{i}}^{2}}\left[\begin{array}{cc}
\left(\varepsilon_{r_{i}} k_{0}{ }^{2}-k_{x}^{2}\right) & k_{x} k_{y} \\
k_{x} k_{y} & \left(\varepsilon_{r_{i}} k_{0}{ }^{2}-k_{y}^{2}\right)
\end{array}\right] \text {, }
$$

com

$$
\alpha_{i}=\frac{k_{0}}{k_{z_{i}} \operatorname{senh}\left(k_{z_{i}} d_{i}\right)} .
$$




\section{B \\ Estudo da segmentação para a função base com condição de borda}

As densidades de corrente superficial ao longo de $x$ e $y$ para funções base com condição de borda segmentada $(\kappa=0,8)$ podem ser visualizadas na Figura B.1 e o campo elétrico espalhado na Figura B.2.

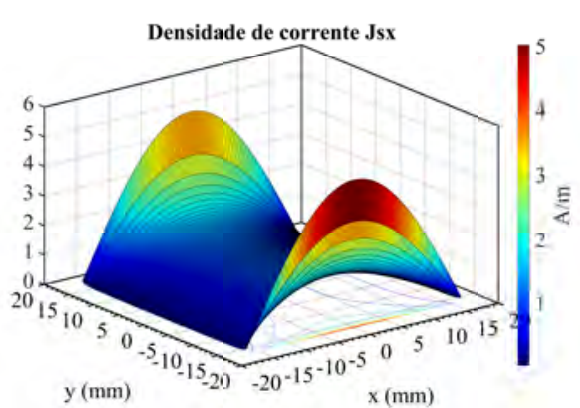

a)

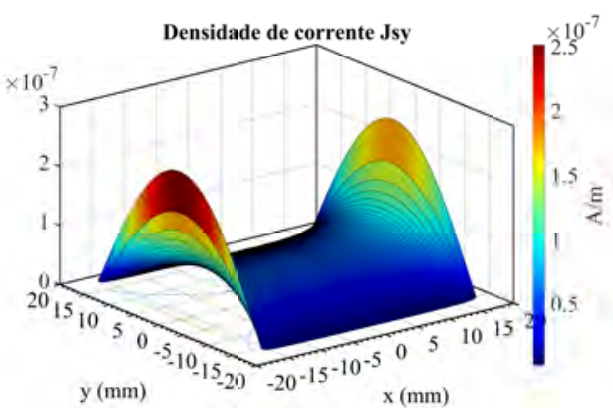

b)

Figura B.1: Densidade de corrente superficial para funções base com condição de borda segmentada, $\kappa=0,8$. a) $J_{x}(x, y)$; b) $J_{y}(x, y)$. 
Apêndice B. Estudo da segmentação para a função base com condição de borda

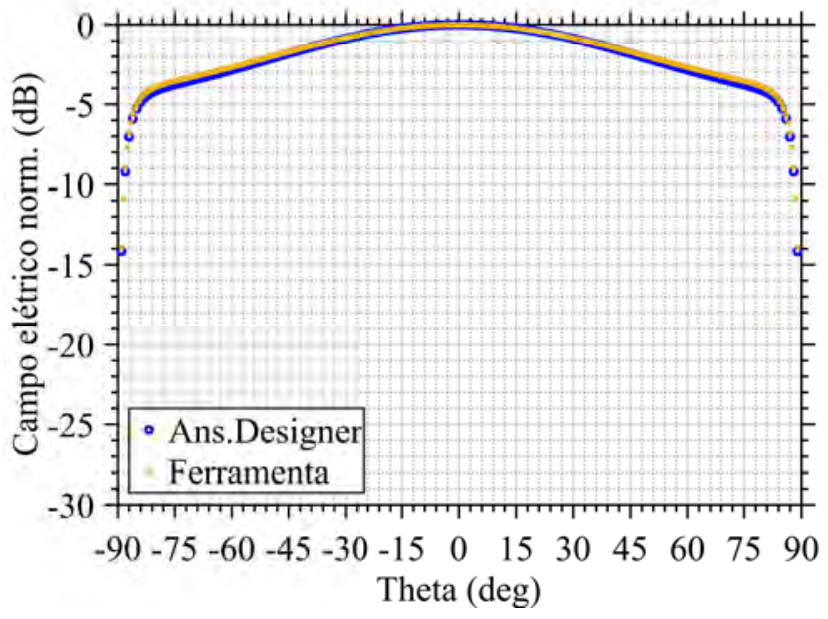

a)

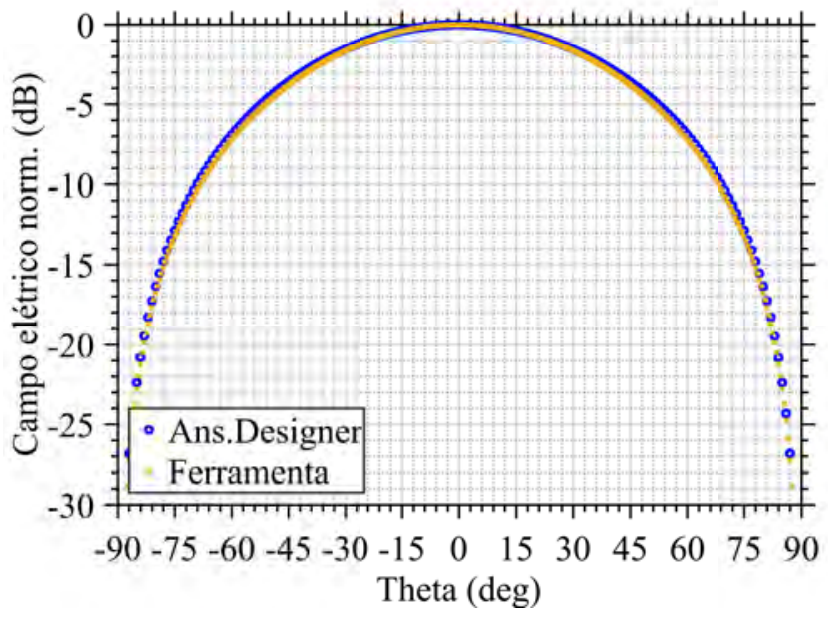

b)

Figura B.2: Campo calculado e simulado para funções base com condição de borda segmentada, $\kappa=0,8$. a) Plano $\mathrm{E}$ : $E_{\theta}\left(\theta, \phi=0^{\circ}\right)$; b) Plano $\mathrm{H}$ : $E_{\phi}\left(\theta, \phi=90^{\circ}\right)$.

As densidades de corrente superficial ao longo de $x$ e $y$ para funções base com condição de borda segmentada $(\kappa=0,6)$ podem ser visualizadas na Figura B.3 e o campo elétrico espalhado na Figura B.4. 
Apêndice B. Estudo da segmentação para a função base com condição de borda

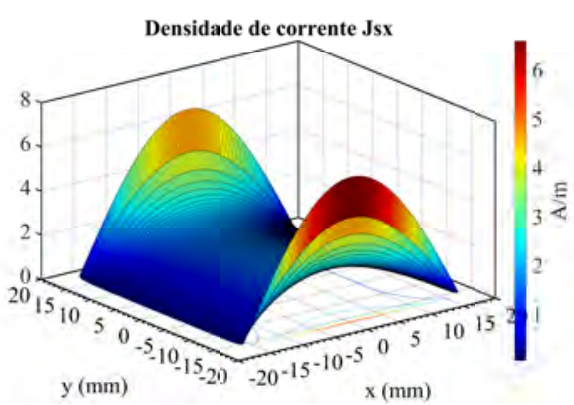

a)

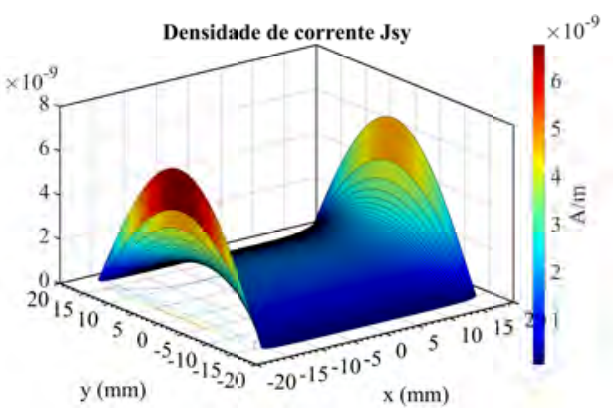

b)

Figura B.3: Densidade de corrente superficial para funções base com condição de borda segmentada, $\kappa=0,6$. a) $J_{x}(x, y)$; b) $J_{y}(x, y)$.

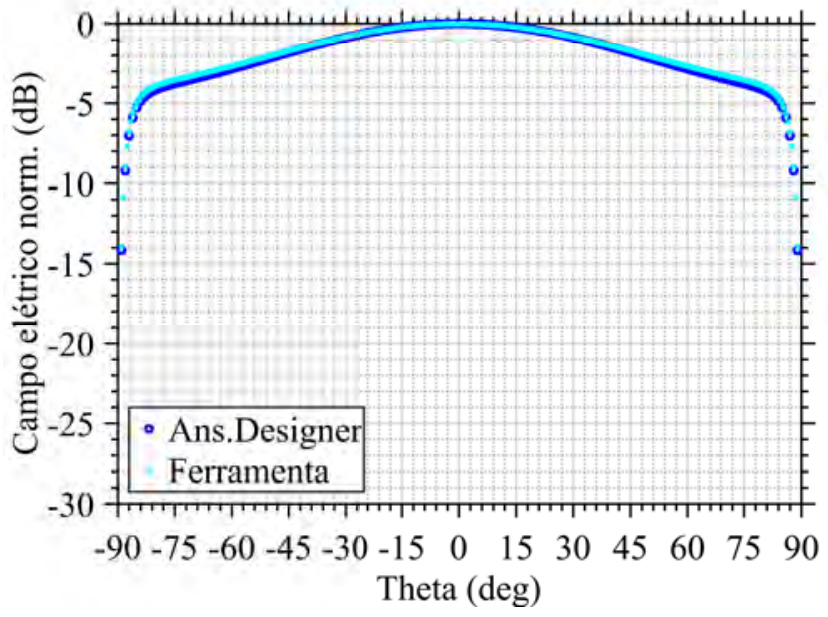

a)

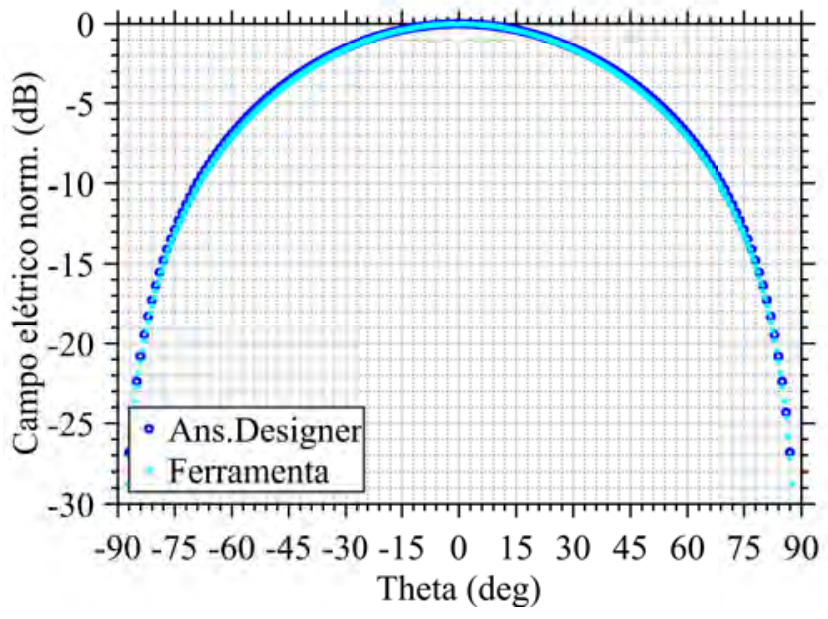

b)

Figura B.4: Campo calculado e simulado para funções base com condição de borda segmentada, $\kappa=0,6$. a) Plano $\mathrm{E}$ : $E_{\theta}\left(\theta, \phi=0^{\circ}\right)$; b) Plano $\mathrm{H}$ : $E_{\phi}\left(\theta, \phi=90^{\circ}\right)$. 
Apêndice B. Estudo da segmentação para a função base com condição de borda

As densidades de corrente superficial ao longo de $x$ e $y$ para funções base com condição de borda segmentada $(\kappa=0,2)$ podem ser visualizadas na Figura B.5 e o campo elétrico espalhado na Figura B.6.

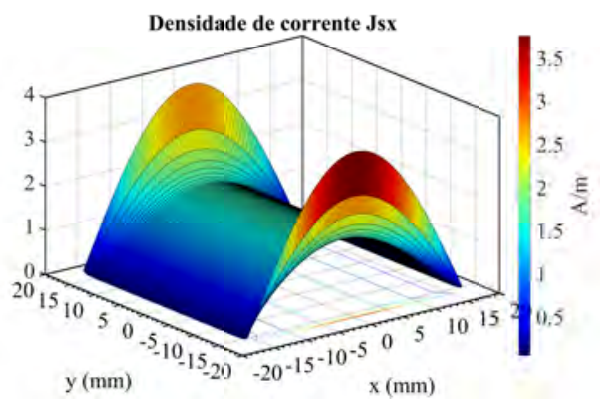

a)

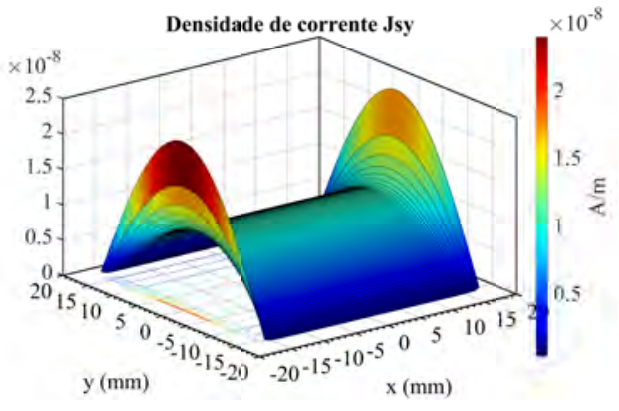

b)

Figura B.5: Densidade de corrente superficial para funções base com condição de borda segmentada, $\kappa=0,2$. a) $J_{x}(x, y)$; b) $J_{y}(x, y)$. 
Apêndice B. Estudo da segmentação para a função base com condição de borda

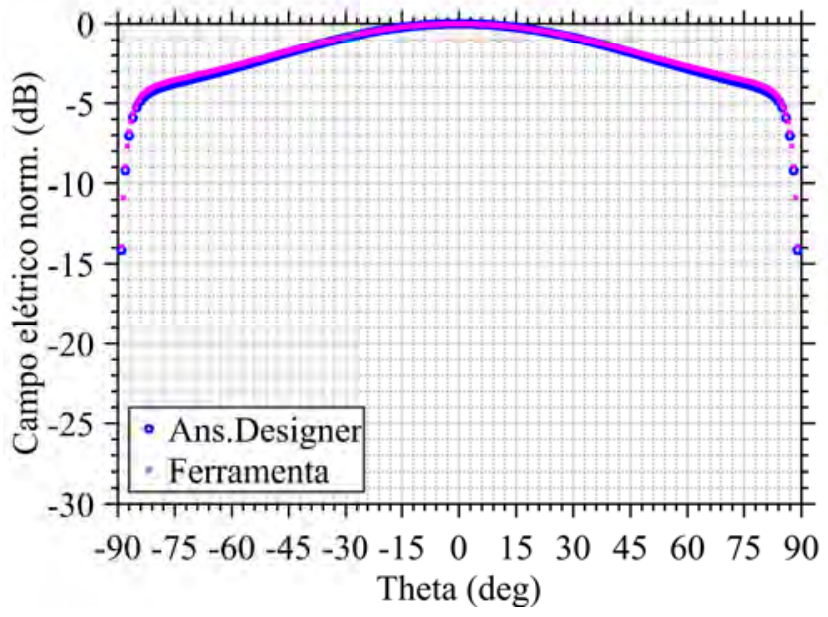

a)

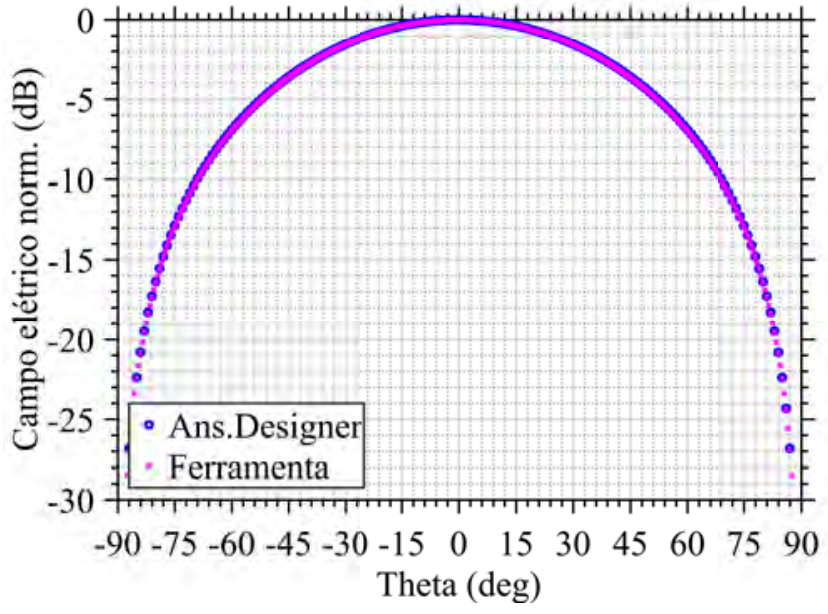

b)

Figura B.6: Campo calculado e simulado para funções base com condição de borda segmentada, $\kappa=0,2$. a) Plano $\mathrm{E}: E_{\theta}\left(\theta, \phi=0^{\circ}\right)$; b) Plano $\mathrm{H}$ : $E_{\phi}\left(\theta, \phi=90^{\circ}\right)$. 